\title{
Coincidências em codimensão um e bordismo
}

\author{
Gustavo de Lima Prado
}

TESE APRESENTADA

$\mathrm{AO}$

Instituto DE MATEMÁtica E EstatísticA

DA

Universidade de SÃo Paulo

PARA

OBTENÇÃO DO TÍTULO

DE

Doutor EM CIÊNCIAS

Programa: Matemática

Orientador: Prof. Dr. Daciberg Lima Gonçalves Coorientador: Prof. Dr. Ulrich Koschorke

Durante o desenvolvimento deste trabalho o autor recebeu auxílio financeiro do $\mathrm{CNPq}$

São Paulo, fevereiro de 2015 



\section{Coincidências em codimensão um e bordismo}

Esta versão da tese contém as correções e alterações sugeridas pela Comissão Julgadora durante a defesa da versão original do trabalho, realizada em 11/02/2015. Uma cópia da versão original está disponível no Instituto de Matemática e Estatística da Universidade de São Paulo.

Comissão Julgadora:

- Prof. Dr. Daciberg Lima Gonçalves (orientador) - IME-USP

- Prof ${ }^{a}$. Dra ${ }^{a}$ Lucília Daruiz Borsari - IME-USP

- Prof ${ }^{\mathrm{a}}$. Dra . Alice Kimie Libardi - UNESP

- Prof. Dr. Oziride Manzoli Neto - ICMC-USP

- Prof. Dr. Pedro Luiz Queiroz Pergher - UFSCar 



\section{Agradecimentos}

Eu agradeço primeiramente a Deus. Na sequência agradeço aos meus orientadores, Daciberg Lima Gonçalves e Ulrich Koschorke, pela disposição, pela paciência, pela bondade, pela hospitalidade, pela riqueza de ideias e pelo tempo (que não foi pouco). Agradeço ainda à minha família, à minha noiva e aos meus amigos por me apoiarem ao longo da jornada. Agradeço por todas as oportunidades, por todas as experiências e por todos os momentos que fizeram de mim quem eu sou e que fizeram deste trabalho a minha tese. 


\section{Resumo}

PRADO, G. L. Coincidências em codimensão um e bordismo. 2014. 135 f. Tese (Doutorado) - Instituto de Matemática e Estatística, Universidade de São Paulo, São Paulo, 2014.

Neste trabalho, estudamos coincidências entre duas aplicações contínuas $f_{1}, f_{2}: X^{n+1} \rightarrow Y^{n}$, onde $X, Y$ são variedades diferenciáveis, conexas, sendo $X$ fechada (compacta e sem bordo) e $Y$ sem bordo. Dizemos que $x \in X$ é uma coincidência do par de aplicações $\left(f_{1}, f_{2}\right)$ se $f_{1}(x)=f_{2}(x)$. Em codimensão um, temos que o conjunto de coincidências $\operatorname{Coin}\left(f_{1}, f_{2}\right)$ é genericamente uma subvariedade de $X$ de dimensão um. Dizemos que $\left(f_{1}, f_{2}\right)$ é deformável a livre de coincidência se existe um par de aplicações $\left(f_{1}^{\prime}, f_{2}^{\prime}\right)$ tal que $f_{i}^{\prime}$ é homotópica a $f_{i}, i=1,2$, e $\operatorname{Coin}\left(f_{1}^{\prime}, f_{2}^{\prime}\right)$ é o conjunto vazio. Em [K-04] e [K-06], Koschorke introduz o invariante de bordismo normal $\tilde{\omega}\left(f_{1}, f_{2}\right) \in \Omega_{1}\left(E\left(f_{1}, f_{2}\right) ; \tilde{\varphi}\right)$, que envolve a subvariedade $\operatorname{Coin}\left(f_{1}, f_{2}\right)$, o qual, em particular, é nulo quando o par de aplicações $\left(f_{1}, f_{2}\right)$ é deformável a livre de coincidência. No caso de codimensão um, quando o domínio é a esfera $\left(X=S^{n+1}\right)$ e quando $\left(f_{1}, f_{2}\right)=\left(y_{0}, f\right)$ (onde $y_{0}$ denota uma aplicação constante), consideramos dois homomorfismos $\omega^{\prime}: \pi_{n+1}(Y) \rightarrow \mathbb{Z}_{2}$ e $\omega^{\prime \prime}: \pi_{n+1}(Y) \rightarrow \pi_{2}(Y)$ que, juntos, sob certas hipóteses, determinam $\tilde{\omega}\left(y_{0}, f\right)$. Neste trabalho, calculamos $\omega^{\prime \prime}: \pi_{n+1}(Y) \rightarrow \pi_{2}(Y)$ para vários espaços e, em particular, para fibrados 
esféricos sobre esferas, obtemos que $\omega^{\prime \prime}=0$ se, e somente se, $Y$ é trivial ou $Y$ não é um $S^{2}$-fibrado sobre $S^{4}$. Agora, um resultado tipo Wecken é um resultado que, sob determinadas condições sobre os espaços, diz que o número mínimo de componentes conexas por caminhos do conjunto de coincidências é igual ao número de Nielsen, isto é, $M C C\left(f_{1}, f_{2}\right)=\tilde{N}\left(f_{1}, f_{2}\right)$, para todo $\left(f_{1}, f_{2}\right)$. Em [K-06], Koschorke obtém resultados tipo Wecken para $n=1$ e para a dimensão estável (que, no caso de codimensão um, significa $n \geqslant 4$ ). Neste trabalho, em codimensão um, obtemos, quando o domínio é a esfera $\left(X=S^{n+1}\right)$, que existe um resultado tipo Wecken para $n=2,3$. Portanto, em codimensão um, quando o domínio é a esfera, existe um resultado tipo Wecken para toda dimensão. Finalmente, também estudamos raízes quando o domínio é o espaço projetivo real $\mathbb{R} P(3)$ e o contradomínio é a esfera $S^{2}$ e também obtemos um resultado tipo Wecken neste caso.

Palavras-chave: coincidência, bordismo normal, codimensão um, propriedade de Wecken, raiz, fibração, cobordismo normal. 


\section{Abstract}

PRADO, G. L. Coincidences in codimension one and bordism. 2014. 135 f. Tese (Doutorado) - Instituto de Matemática e Estatística, Universidade de São Paulo, São Paulo, 2014.

In this work, we study coincidences between two maps $f_{1}, f_{2}: X^{n+1} \rightarrow$ $Y^{n}$, where $X, Y$ are smooth manifolds, connected, being $X$ closed (compact and without boundary) and $Y$ without boundary. We say that $x \in X$ is a coincidence of the pair of maps $\left(f_{1}, f_{2}\right)$ if $f_{1}(x)=f_{2}(x)$. In codimension one we have that the coincidence set $\operatorname{Coin}\left(f_{1}, f_{2}\right)$ is generically a submanifold of $X$ of dimension one. We say that $\left(f_{1}, f_{2}\right)$ is loose if there exists a pair of maps $\left(f_{1}^{\prime}, f_{2}^{\prime}\right)$ such that $f_{i}^{\prime}$ is homotopic to $f_{i}, i=1,2$, and $\operatorname{Coin}\left(f_{1}^{\prime}, f_{2}^{\prime}\right)$ is the empty set. In [K-04] and [K-06], Koschorke introduces the normal bordism invariant $\tilde{\omega}\left(f_{1}, f_{2}\right) \in \Omega_{1}\left(E\left(f_{1}, f_{2}\right) ; \tilde{\varphi}\right)$, involving the submanifold $\operatorname{Coin}\left(f_{1}, f_{2}\right)$, that, in particular, is null when the pair of maps $\left(f_{1}, f_{2}\right)$ is loose. In the codimension one case, when the domain is the sphere $\left(X=S^{n+1}\right)$, and when $\left(f_{1}, f_{2}\right)=$ $\left(y_{0}, f\right)$ (where $y_{0}$ denotes a constant map), we consider two homomorphisms $\omega^{\prime}: \pi_{n+1}(Y) \rightarrow \mathbb{Z}_{2}$ and $\omega^{\prime \prime}: \pi_{n+1}(Y) \rightarrow \pi_{2}(Y)$ which together, under some hypothesis, determine $\tilde{\omega}\left(y_{0}, f\right)$. In this work, we calculate $\omega^{\prime \prime}: \pi_{n+1}(Y) \rightarrow$ $\pi_{2}(Y)$ for several spaces and, in particular, for sphere bundles over spheres we obtain that $\omega^{\prime \prime}=0$ if and only if $Y$ is trivial or $Y$ is not an $S^{2}$-bundle 
over $S^{4}$. Now, a Wecken type result is a result which, under some conditions about the spaces, asserts that the minimum number of path-components of the coincidence set is equal to the Nielsen number, that is, $\operatorname{MCC}\left(f_{1}, f_{2}\right)=$ $\tilde{N}\left(f_{1}, f_{2}\right)$ for all $\left(f_{1}, f_{2}\right)$. In [K-06], Koschorke obtains Wecken type results for $n=1$ and for the stable dimension (which, in the codimension one case, means $n \geqslant 4)$. In this work, in codimension one, we obtain, when the domain is the sphere $\left(X=S^{n+1}\right)$, that there exists a Wecken type result for $n=2,3$. Hence, in codimension one, when the domain is the sphere, there exists a Wecken type result for every dimension. Finally, we also study roots when the domain is the real projective space $\mathbb{R} P(3)$ and the target space is the sphere $S^{2}$ and we also obtain a Wecken type result in this case.

Keywords: coincidence, normal bordism, codimension one, Wecken property, root, fibration, normal cobordism. 


\section{Índice}

$\begin{array}{ll}\text { Introdução } & 1\end{array}$

1 Preliminares 4

1.1 Convenções e notações . . . . . . . . . . . . . . . . . . . . . . 4

1.2 Sobre fibrações e fibrados . . . . . . . . . . . . . . . . . . 5

1.3 Sobre CW-complexos . . . . . . . . . . . . . 16

1.4 Sobre variedades . . . . . . . . . . . . . . . . . . . 19

1.5 Coincidências . . . . . . . . . . . . . . . . . 21

1.6 Topologia diferencial . . . . . . . . . . . . . . . . . . . . . 28

1.7 Bordismo ........................... 31

1.8 Bordismo e coincidências . . . . . . . . . . . . . . . 37

1.8.1 Sobre o invariante $\omega\left(f_{1}, f_{2}\right) \ldots \ldots . \ldots 37$

1.8.2 Sobre o invariante $\tilde{\omega}\left(f_{1}, f_{2}\right) \quad \ldots \ldots . \ldots . . \ldots 38$

1.8.3 Sobre o número de Nielsen $\tilde{N}\left(f_{1}, f_{2}\right) \ldots \ldots$. . . . . . 40

1.8.4 Resultados tipo Wecken . . . . . . . . . . . . 41

1.8.5 Raízes e bordismo . . . . . . . . . . . . . . . . 42

1.8.6 Auto-coincidências e bordismo . . . . . . . . . . 44

2 Coincidências em codimensão 1 , sendo o domínio uma esfera 46

2.1 Particularidades sobre o invariante . . . . . . . . . . . 47 
2.2 Cálculos do invariante . . . . . . . . . . . . . . . 56

2.2.1 $\omega^{\prime \prime}$ para esferas . . . . . . . . . . . . . 57

2.2.2 $\omega^{\prime \prime}$ para espaços projetivos reais . . . . . . . . 58

2.2.3 $\omega^{\prime \prime}$ para superfícies e 3-variedades . . . . . . . . . . 59

2.2.4 Sobre o homomorfismo $\omega^{\prime} \ldots \ldots$. . . . . . . . . 60

2.3 Um teorema tipo Wecken . . . . . . . . . . . . . . . 62

2.4 Mais cálculos de $\omega^{\prime \prime} \ldots$. . . . . . . . . . . . . . . . . 64

2.4.1 Espaços projetivos complexos e quaterniônicos . . . . . 65

2.4.2 Fibrados esféricos sobre esferas . . . . . . . . 66

2.4.3 Variedades de Stiefel . . . . . . . . . . . . . . . 83

2.5 Cálculos parciais de $\omega^{\prime \prime} \ldots \ldots$. . . . . . . . . . . . 85

2.5.1 4-variedades . . . . . . . . . . . . 86

2.5.2 $F^{a}$-fibrados sobre $B^{b} \ldots \ldots \ldots$. . . . . . . . 88

2.5.3 Variedades de Grassmann . . . . . . . . . . . . . . . 92

3 Raízes em codimensão 1, sendo o domínio uma 3-variedade 96

$3.1 X$ uma forma espacial esférica e $\chi(Y) \leqslant 0 \ldots \ldots 7$

$3.2 \quad X=\mathbb{R} P(3)$ e $Y=S^{2} \ldots \ldots \ldots \ldots$. . . . . . . . 98

3.2.1 Particularidades sobre o invariante . . . . . . . . 98

3.2.2 Aplicações contínuas de $\mathbb{R} P(3)$ em $S^{2}$. . . . . . . . . 99

3.2.3 Cálculos do invariante . . . . . . . . . . . 106

Referências Bibliográficas 


\section{Introdução}

Neste trabalho, estudamos coincidências em codimensão um, usando bordismo normal. Dadas aplicações contínuas $f_{1}, f_{2}: X \rightarrow Y$, na teoria de coincidência, estamos interessados em coincidências entre $f_{1}$ e $f_{2}$, isto é, em pontos $x \in X$ tais que $f_{1}(x)=f_{2}(x)$. Mais especificamente, aqui estamos interessados no comportamento do conjunto de coincidências $\operatorname{Coin}\left(f_{1}^{\prime}, f_{2}^{\prime}\right)$, onde $f_{i}^{\prime}$ é homotópica a $f_{i}, i=1,2$. Por exemplo, ora queremos saber se $\left(f_{1}, f_{2}\right)$ é deformável a livre de coincidência (isto é, se existe $\left(f_{1}^{\prime}, f_{2}^{\prime}\right)$ homotópica a $\left(f_{1}, f_{2}\right)$ tal que $\left.\operatorname{Coin}\left(f_{1}^{\prime}, f_{2}^{\prime}\right)=\emptyset\right)$, ora queremos calcular o número mínimo de coincidências $M C\left(f_{1}, f_{2}\right):=\min \left\{\# \operatorname{Coin}\left(f_{1}^{\prime}, f_{2}^{\prime}\right) \mid f_{i}^{\prime} \simeq f_{i}\right\}$, ora queremos calcular o número mínimo de componentes de coincidências, $\operatorname{MCC}\left(f_{1}, f_{2}\right):=\min \left\{\# \pi_{0}\left(\operatorname{Coin}\left(f_{1}^{\prime}, f_{2}^{\prime}\right)\right) \mid f_{i}^{\prime} \simeq f_{i}\right\}$.

Um avanço significativo na teoria de coincidência se deu entre 1923 e 1927, quando Lefschetz ([L-23],[L-25],[L-26],[L-27]) deu condições suficientes para a existência de coincidência, para variedades orientáveis fechadas de mesma dimensão. Após este resultado, vários outros seguiram para a teoria de ponto fixo, inclusive um resultado devido a Wecken ([We-41],[We-412],[We-42]), que diz que, sob determinadas hipóteses sobre o espaço, uma auto-aplicação de um complexo simplicial sempre pode ser deformada a uma outra com exatamente o número de Nielsen de pontos fixos. Em 1955, Schirmer ([Sc-55]), em sua tese, demonstrou um resultado tipo Wecken para coincidência, para 
variedades orientáveis fechadas de mesma dimensão maior ou igual a 3. Até recentemente, as questões para coincidência têm sido estudadas predominantemente em codimensão zero, isto é, quando os espaços envolvidos têm a mesma dimensão. Para maiores detalhes, vide [Brow-99] e [Go-05].

Este trabalho está dividido em 3 capítulos. No capítulo 1, apresentamos definições e resultados gerais que são utilizados por todo o trabalho. Em particular, expomos um pouco das teorias de coincidência, de auto-coincidência, de raiz e um pouco da teoria de bordismo neste capítulo. Daí, introduzimos alguns conceitos e resultados para coincidência, usando bordismo. Por exemplo, apresentamos, para pares $\left(f_{1}, f_{2}\right)$ de aplicações contínuas, os invariantes $\omega\left(f_{1}, f_{2}\right)$ e $\tilde{\omega}\left(f_{1}, f_{2}\right)$ (que pertencem a grupos de bordismo normal), os quais necessariamente se anulam quando o par de aplicações contínuas é deformável a livre de coincidência (vide [K-04] e [K-06]).

No capítulo 2 , estamos interessados em estudar o invariante $\tilde{\omega}\left(f_{1}, f_{2}\right)$ quando o domínio das aplicações é uma esfera $(n+1)$-dimensional e o contradomínio é uma variedade sem bordo, conexa, $n$-dimensional, lembrando que tal invariante pertence ao grupo de bordismo normal $\Omega_{1}\left(E\left(f_{1}, f_{2}\right) ; \tilde{\varphi}\right)$. Neste contexto, veremos que tal grupo é isomorfo a $\Omega_{1}^{f r}\left(\Omega\left(Y, y_{0}\right)\right)$, deixando então de depender do par $\left(f_{1}, f_{2}\right)$ de aplicações contínuas e do fibrado virtual $\tilde{\varphi}$. Além disso, veremos que é possível decompor $\tilde{\omega}\left(f_{1}, f_{2}\right)$ na soma de $\tilde{\omega}\left(f_{1}, f_{1}\right)$, no caso especial de auto-coincidências, com $\tilde{\omega}\left(y_{0}, f_{2}-f_{1}\right)$, no caso especial de raízes. Dando mais ênfase a este último, definiremos dois homomorfismos $\omega^{\prime}: \pi_{n+1}(Y) \rightarrow \mathbb{Z}_{2}$ e $\omega^{\prime \prime}: \pi_{n+1}(Y) \rightarrow \pi_{2}(Y)$ que ajudam no cálculo do número de Nielsen $\tilde{N}\left(y_{0}, f\right)$, para toda aplicação contínua $f: S^{n+1} \rightarrow Y$. Daí, estudando tais homomorfismos para superfícies e 3-variedades, nós obtemos um resultado tipo Wecken para $n=2,3$ (teorema 159), o qual diz que o número mínimo de componentes de coincidências $\operatorname{MCC}\left(f_{1}, f_{2}\right)$ é igual ao número de 
Nielsen $\tilde{N}\left(f_{1}, f_{2}\right)$, para quaisquer aplicações contínuas $f_{1}, f_{2}: S^{n+1} \rightarrow Y$, com $n=2,3$. Daí, juntando com resultados tipo Wecken de Koschorke ([K-06]), enunciamos tal resultado para toda dimensão $n \geqslant 1$. Por fim, nós calculamos $\omega^{\prime \prime}$ para vários espaços $Y$ (proposições 151, 152, 153, 164, 165, 178, entre outros resultados) e, em particular, nós obtemos que tal homomorfismo é identicamente nulo para todo fibrado esférico sobre esfera, com exceção de $S^{2}$-fibrados não triviais sobre $S^{4}$, quando tal homomorfismo, restrito a $\mathbb{Z}$, é injetor (proposição 166 e teorema 171).

No capítulo 3, estamos interessados em estudar, para o par $\left(y_{0}, f\right)$, quando o número mínimo de componentes de coincidências $M C C\left(y_{0}, f\right)$ é igual ao número de Nielsen $\tilde{N}\left(y_{0}, f\right)$, isto é, queremos saber quando o par $\left(y_{0}, f\right)$ satisfaz a propriedade de Wecken, supondo que o domínio $X$ de $f$ é uma 3-variedade fechada, conexa e o contradomínio $Y$ de $f$ é uma superfície fechada, conexa. Mais especificamente, neste capítulo, estudamos a validade da propriedade de Wecken em algumas situações quando $X$ é uma forma espacial esférica, a saber: sendo $X$ uma forma espacial esférica qualquer e $Y=K(\pi, 1)$, nós obtemos que $M C C\left(y_{0}, f\right)=N\left(y_{0}, f\right)=0$ sempre (proposição 192); e sendo $X=\mathbb{R} P(3)$ e $Y=S^{2}$, nós classificamos, a menos de homotopia, as aplicações contínuas (teorema 196) e nós obtemos que $\operatorname{MCC}\left(y_{0}, f\right)=N\left(y_{0}, f\right)=1$ se $f$ não é homotópica a aplicação constante e $M C C\left(y_{0}, f\right)=N\left(y_{0}, f\right)=0$, caso contrário (teorema 206). 


\section{Capítulo 1}

\section{Preliminares}

\subsection{Convenções e notações}

Por variedade, entenderemos variedade $C^{\infty}$ e por aplicação diferenciável, entenderemos aplicação diferenciável $C^{\infty}$. Ainda, aqui, uma variedade é suposta ser um espaço topológico Hausdorff satisfazendo o segundo axioma de enumerabilidade. Denotaremos o bordo de uma variedade $M$ por $\partial M$ e se $M$ é uma variedade sem bordo, escrevemos $\partial M=\emptyset$. Por variedade fechada, entenderemos variedade compacta, sem bordo e por variedade aberta, entenderemos variedade não compacta, sem bordo. Ainda, em geral, denotaremos uma variedade $M$ de dimensão $m$ por $M^{m}$. Denotaremos por $I$ o intervalo compacto $[0,1]$.

Denotaremos por $p r_{i}, i=1,2$, a projeção de um produto cartesiano (ou de um subconjunto deste) no $i$-ésimo fator. Denotaremos a aplicação identidade de $X$ por $1_{X}$ e uma aplicação constante igual a $x \in X$ por $c_{x}$. Ainda, dado um ponto $x \in X, x$ denotará tanto o próprio ponto, como o conjunto unitário $\{x\}$, como a aplicação constante $c_{x}$. Para um conjunto $X$, denotaremos a cardinalidade de $X$, em geral, por \#X. Ainda, denotaremos a união disjunta 
por ப்.

Denotaremos as relações entre espaços homeomorfos por $\approx$, espaços com o mesmo tipo de homotopia por $\sim$, espaços isomorfos por $\cong$ e aplicações homotópicas por $\simeq$. Se $H: X \times I \rightarrow Y$ é uma homotopia, denotaremos $H_{t}:=$ $H\left({ }_{-}, t\right)$, para todo $t \in I$. Ainda, denotaremos por $H: f \simeq g$ uma homotopia $H$ tal que $H_{0}=f$ e $H_{1}=g$. Denotaremos por $\alpha \simeq_{\partial I} \beta$ uma homotopia com extremos fixados, sendo $\alpha, \beta: I \rightarrow X$ caminhos. Denotaremos por $\pi_{n}(X)$ o grupo $\pi_{n}\left(X, x_{0}\right)$, para algum $x_{0} \in X$, lembrando que tal grupo em geral depende do ponto base, uma vez que é calculado na componente conexa por caminhos que contém tal ponto. Da mesma forma, denotaremos por $\pi_{0}(X)$ o conjunto $\pi_{0}\left(X, x_{0}\right)$, para algum $x_{0} \in X$. Finalmente, denotaremos o conjunto das classes de homotopia das aplicações contínuas de $X$ em $Y$ por $[X, Y]$.

\subsection{Sobre fibrações e fibrados}

Em toda a seção, $E, B$ e $F$ são espaços topológicos e $p: E \rightarrow B$ é uma aplicação contínua.

Nesta seção, apresentamos definições e resultados sobre fibrações e fibrados, além de vários exemplos, que são utilizados por todo o trabalho.

Definição 1 (Propriedade do levantamento de homotopia). Seja X um espaço topológico. Dizemos que p satisfaz a propriedade do levantamento de homotopia para $X$, se, dadas $F: X \times I \rightarrow B$ e $\tilde{f}: X \rightarrow E$ aplicações contínuas tais que $p \circ \tilde{f}(x)=F(x, 0)$, existe $\tilde{F}: X \times I \rightarrow E$ aplicação continua tal que $p \circ \tilde{F}=F$ e $\tilde{F}(x, 0)=\tilde{f}(x)$, para todo $x \in X$.

Considerando $\tilde{f}: X \times 0 \rightarrow E, \tilde{f}(x, 0):=\tilde{f}(x)$, para todo $x \in X$, obtemos o 
seguinte diagrama geométrico

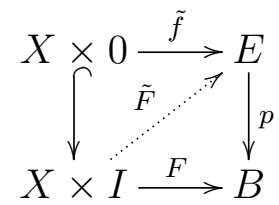

Definição 2. Dizemos que a tripla $(E, p, B)$, ou a aplicação $p: E \rightarrow B$, é uma fibração (de Hurewicz), se p satisfaz a propriedade do levantamento de homotopia, para todo espaço topológico $X$.

Chamamos $E, B$ e $p^{-1}(b)$, com $b \in B$, respectivamente, de espaço total, espaço base e fibra (sobre b) da fibração. Denotamos por $F \rightarrow E \stackrel{p}{\rightarrow} B$, uma fibração $p: E \rightarrow B$, com $F=p^{-1}\left(b_{0}\right)$, para algum $b_{0} \in B$.

Exemplo 3. A projeção, $p r_{1}$, do produto cartesiano $B \times F$ em $B$ é uma fibração, chamada de fibração trivial.

Definição 4. Seja $f: B^{\prime} \rightarrow B$ uma aplicação contínua. Definimos o pullback de $f$ e p como sendo o espaço topológico $E^{\prime}:=\left\{\left(b^{\prime}, e\right) \in B^{\prime} \times E: f\left(b^{\prime}\right)=p(e)\right\}$ junto com aplicações contínuas $p^{\prime}, f^{\prime}$ dadas por $p^{\prime}\left(b^{\prime}, e\right)=b^{\prime}$ e $f^{\prime}\left(b^{\prime}, e\right)=$ e. Notemos que $p \circ f^{\prime}=f \circ p^{\prime}$, ou seja, o diagrama geométrico abaixo é comutativo.

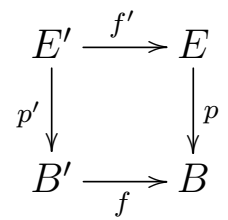

Observação 5. Notemos que o pullback de $f$ e p tem a seguinte propriedade universal: para todo espaço topológico $E^{\prime \prime}$ junto com aplicações contínuas $f^{\prime \prime}: E^{\prime \prime} \rightarrow E$ e $p^{\prime \prime}: E^{\prime \prime} \rightarrow B^{\prime}$ tais que $p \circ f^{\prime \prime}=f \circ p^{\prime \prime}$, existe uma única 
aplicação contínua $h: E^{\prime \prime} \rightarrow E^{\prime}$ tal que $f^{\prime} \circ h=f^{\prime \prime}$ e $p^{\prime} \circ h=p^{\prime \prime}$.

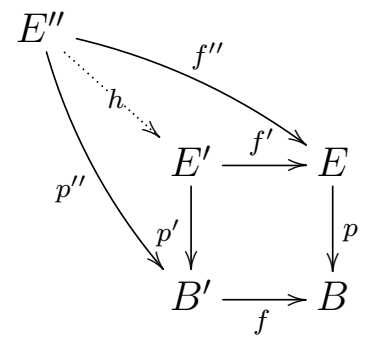

Exemplo 6. Com as notações da definição 4, se p:E $\rightarrow$ B é uma fibração, então $f^{*}(p):=p^{\prime}: E^{\prime} \rightarrow B^{\prime}$ também é uma fibração, chamada de fibração induzida de p por $f$, ou de pullback de p por $f$. Ainda, chamamos $f^{*}(E):=$ $E^{\prime}$ de pullback de $E$ (ou de $p$ ) por $f$.

Definição 7. Sejam $p_{1}: E_{1} \rightarrow B, p_{2}: E_{2} \rightarrow B$ fibrações. Dizemos que uma aplicação contínua $g: E_{1} \rightarrow E_{2}$ preserva fibra sobre $B$, se $p_{2} \circ g=p_{1}$. Dizemos que uma homotopia $H: E_{1} \times I \rightarrow E_{2}$ preserva fibra sobre $B$, se $p_{2} \circ H_{t}=p_{1}$, para todo $t \in I$. Dizemos que $p_{1}$ e $p_{2}$, ou $E_{1}$ e $E_{2}$, têm o mesmo tipo de homotopia sobre $B$, ou têm o mesmo tipo de homotopia via fibra, se existem aplicações contínuas $g_{1}: E_{1} \rightarrow E_{2}, g_{2}: E_{2} \rightarrow E_{1} e$ homotopias $H_{1}: g_{2} \circ g_{1} \simeq 1_{E_{1}}, H_{2}: g_{1} \circ g_{2} \simeq 1_{E_{2}}$ que preservam fibra sobre B. Neste caso, $g_{1}, g_{2}$ são chamadas de equivalências de homotopia sobre $B$, ou equivalências de homotopia via fibra.

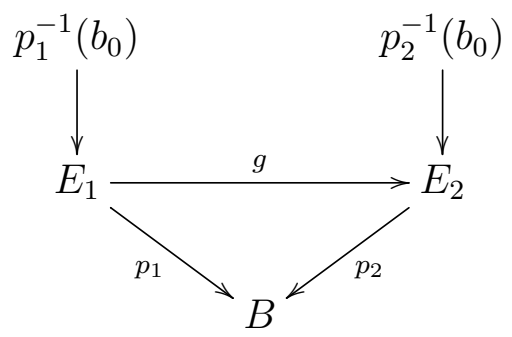

Ainda, dizemos que $E_{1}$ e $E_{2}$ são homeomorfos sobre $B$, se existe um homomorfismo $g: E_{1} \rightarrow E_{2}$ que preserva fibra sobre $B$. 
Teorema 8. Sejam $f_{1}, f_{2}: B^{\prime} \rightarrow B$ aplicações contínuas tais que $f_{1} \simeq f_{2}$. Se $p: E \rightarrow B$ é uma fibração, então os pullbacks $f_{1}^{*}(p)$ e $f_{2}^{*}(p)$ têm o mesmo tipo de homotopia sobre $B^{\prime}$.

Tal resultado pode ser encontrado em [Sp-66] (teorema 14, página 102).

Definição 9. Seja $p: E \rightarrow B$ uma fibração. Uma seção (ou seção transversal) de p é uma aplicação contínua $s: B \rightarrow E$ tal que $p \circ s=1_{B}$.

Observação 10. Seja $(E, p, B)$ uma fibração. Sejam $b_{0} \in B$ e $F:=p^{-1}\left(b_{0}\right)$. Seja $n \in \mathbb{Z}, n \geqslant 0$. Dada $[g] \in \pi_{n+1}\left(B, b_{0}\right)$, com $g:\left(D^{n+1}, S^{n}\right) \rightarrow\left(B, b_{0}\right)$, consideramos $g^{\prime}:\left(\frac{S^{n} \times I}{S^{n} \times 0}, S^{n} \times 1\right) \rightarrow\left(B, b_{0}\right), g^{\prime} \overline{(x, t)}:=g(t x)$, para todo $(x, t) \in$ $S^{n} \times I$. Como p é uma fibração, existe $\tilde{G}: \frac{S^{n} \times I}{S^{n} \times 0} \rightarrow E$ tal que $p \circ \tilde{G}=g^{\prime}$. Daí, $\left[\tilde{G}_{1}\right] \in \pi_{n}(F)$ e definimos $\partial_{n+1}: \pi_{n+1}(B) \rightarrow \pi_{n}(F)$ como sendo dada por $\partial_{n+1}([g])=\left[\tilde{G}_{1}\right]$.

Teorema 11. Se $F \rightarrow E \stackrel{p}{\rightarrow} B$ é uma fibração, então existe uma sequência exata

$$
\begin{gathered}
\cdots \longrightarrow \pi_{n+1}(B) \stackrel{\partial_{n+1}}{\longrightarrow} \pi_{n}(F) \stackrel{i_{\#}}{\longrightarrow} \pi_{n}(E) \stackrel{p_{\#}}{\longrightarrow} \pi_{n}(B) \stackrel{\partial_{n}}{\longrightarrow} \pi_{n-1}(F) \longrightarrow \cdots \\
\cdots \pi_{2}(B) \stackrel{\partial_{2}}{\longrightarrow} \pi_{1}(F) \stackrel{i_{\#}}{\longrightarrow} \pi_{1}(E) \stackrel{p_{\#}}{\longrightarrow} \pi_{1}(B) \stackrel{\partial_{1}}{\longrightarrow} \pi_{0}(F) \longrightarrow \\
\longrightarrow \pi_{0}(E) \stackrel{p_{\#}}{\longrightarrow} \pi_{0}(B)
\end{gathered}
$$

chamada de sequência de homotopia da fibração $p$, onde $i: F \hookrightarrow E$ é a inclusão e $\partial_{n+1}$ é como acima, para todo $n \in \mathbb{Z}, n \geqslant 0$.

Tal resultado pode ser encontrado em [Sp-66] (teorema 10, página 377). Observemos que, se $X$ é um espaço topológico, então $\pi_{n}(X)$ é um grupo para $n \geqslant 1$ e, em geral, um conjunto para $n=0$. Na verdade, $\pi_{n}(X)$ é um grupo abeliano para $n \geqslant 2$. Em particular, a sequência acima é uma sequência de grupos e homomorfismos de grupos em geral até $\pi_{1}(B)$. 
Definição 12. Dizemos que a quádrupla $(E, p, B, F)$, ou a aplicação $p$ : $E \rightarrow B$, ou o espaço $E$, é um fibrado (localmente trivial) sobre $B$ com fibra $F$, ou um F-fibrado sobre $B$, se existe uma cobertura aberta $\mathcal{C}$ de $B$ e, para cada $U \in \mathcal{C}$, existe um homeomorfismo $\varphi_{U}: U \times F \rightarrow p^{-1}(U)$ tal que $p \circ \varphi_{U}: U \times F \rightarrow U$ é a projeção no primeiro fator. Notemos que $p^{-1}(b) \approx F$, para todo $b \in B$.

Chamamos E, B, F e p, respectivamente, de espaço total, espaço base, fibra e projeção do fibrado.

Observação 13. Notemos que podemos ver $E$ como sendo $\bigcup_{b \in B} p^{-1}(b)$ ou $\bigcup_{b \in B}\{b\} \times p^{-1}(b)$.

Observação 14. Se $\xi$ é um fibrado, denotamos $\xi:=\left(E(\xi), p_{\xi}, B(\xi), F(\xi)\right)$.

Exemplo 15. A quádrupla $\left(B \times F, p r_{1}, B, F\right)$ é um fibrado, chamado de fibrado produto.

Exemplo 16. Se $p: \tilde{X} \rightarrow X$ é uma projeção de revestimento, com $X$ conexo, então, dado $x_{0} \in X$, a quádrupla $\left(\tilde{X}, p, X, p^{-1}\left(x_{0}\right)\right)$ é um fibrado, com fibra discreta.

Exemplo 17. Com as notações da definição 4, se $\xi=(E, p, B, F)$ é um fibrado, então $f^{*}(\xi):=\left(E^{\prime}, p^{\prime}, B^{\prime}, F\right)$ também é um fibrado, chamado de fibrado induzido de $\xi$ (ou de p) por $f$, ou de pullback de $\xi$ (ou de p) por $f$. Ainda, chamamos $f^{*}(E):=E^{\prime}$ de pullback de $E$ (ou de $p$, ou de $\xi$ ) por $f$.

Exemplo 18. Sejam $\xi_{1}=\left(E_{1}, p_{1}, B_{1}, F_{1}\right)$ e $\xi_{2}=\left(E_{2}, p_{2}, B_{2}, F_{2}\right)$ fibrados. Então $\xi_{1} \times \xi_{2}:=\left(E_{1} \times E_{2}, p_{1} \times p_{2}, B_{1} \times B_{2}, F_{1} \times F_{2}\right)$ também é um fibrado.

Exemplo 19. Sejam $\xi_{1}, \xi_{2}$ fibrados sobre B. Seja $d: B \rightarrow B \times B, b \mapsto(b, b)$, a aplicação diagonal. Então $\xi_{1} \oplus \xi_{2}:=d^{*}\left(\xi_{1} \times \xi_{2}\right)$ é um fibrado, chamado de soma de Whitney de $\xi_{1}$ e $\xi_{2}$. 
Definição 20. Seja $\operatorname{Homeo}(F)$ o grupo dos homeomorfismos de $F$ em $F$ e seja $G$ um subgrupo de $\operatorname{Homeo}(F)$. Seja $\xi=(E, p, B, F)$ um fibrado. Com as notações da definição 12, dizemos que $\xi$ tem grupo estrutural $G$, se $\varphi_{V, b}^{-1} \circ \varphi_{U, b} \in G$, para todo $b \in U \cap V$, para todo $U, V \in \mathcal{C}$, onde $\varphi_{U, b}, \varphi_{V, b}: F \rightarrow$ $p^{-1}(b)$ são dadas por $x \mapsto \varphi_{U}(b, x), x \mapsto \varphi_{V}(b, x)$.

Exemplo 21. O grupo $\operatorname{Homeo}(F)$ é sempre um grupo estrutural para um fibrado $\xi=(E, p, B, F)$. Se $\xi$ é o fibrado produto, então $\xi$ tem grupo estrutural trivial $G=\left\{1_{F}\right\}$. Se E, B,F são variedades e $p$ é uma aplicação diferenciável, então $\xi$ tem grupo estrutural $\operatorname{Diff}(F)$, o grupo dos difeomorfismos de F em F.

Definição 22. Sejam $\xi_{1}=\left(E_{1}, p_{1}, B, F\right), \xi_{2}=\left(E_{2}, p_{2}, B, F\right)$ fibrados sobre $B$ com fibra $F$. Dizemos que $\xi_{1}, \xi_{2}$ são equivalentes (sobre $B$ ), ou isomorfos (sobre B), se existe um homeomorfismo $h: E_{1} \rightarrow E_{2}$ que preserva fibra sobre $B$, ou seja, que é tal que $p_{2} \circ h=p_{1}$. Escrevemos $\xi_{1} \cong \xi_{2}$. Nesta situação, dizemos que $h: \xi_{1} \rightarrow \xi_{2}$ é um isomorfismo de fibrados sobre B. Ainda, dizemos que um fibrado é trivial, se ele é equivalente ao fibrado produto.

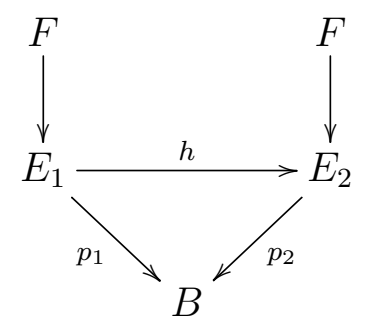

Se $\xi_{1}, \xi_{2}$ têm grupo estrutural $G$, dizemos que eles são equivalentes (sobre $B$, sobre $G$ ) se, além disto, $h_{\mid}: F \rightarrow F$ pertence a $G$.

Definição 23. Seja $\xi=(E, p, B, F)$ um fibrado. Dizemos que $\xi$ é um fibrado vetorial (real) se $F$ é um espaço vetorial real e $\xi$ tem grupo estrutural $G L(F)$, o grupo linear geral de $F$, isto é, o grupo dos isomorfismos de $F$ em 
$F$. Se $\operatorname{dim}_{\mathbb{R}} F=k$, isto é, se $F \cong \mathbb{R}^{k}$, para algum $k \in \mathbb{N}$, dizemos que $\xi$ é um $k$-fibrado vetorial (real) e o denotamos por $\xi^{k}$. Ainda, se $\xi^{k}$ é trivial, então o denotamos por $\mathbb{R}^{k}$ ou simplesmente por $\mathbb{R}$ (quando não houver confusão).

Observação 24. Um isomorfismo $h: \xi_{1} \rightarrow \xi_{2}$ de fibrados vetoriais sobre $B$ é tal que $h: E\left(\xi_{1}\right) \rightarrow E\left(\xi_{2}\right)$ é um homeomorfismo que preserva fibra sobre $B$ e $h_{1}: F\left(\xi_{1}\right) \rightarrow F\left(\xi_{2}\right)$ é um isomorfismo de espaços vetoriais. Sejam, $\xi^{k}, \eta^{l}$ fibrados vetoriais sobre $B$ e $f: B^{\prime} \rightarrow B$ uma aplicação contínua. Então o pullback de $\xi$ por $f$ é um $k$-fibrado vetorial sobre $B^{\prime}$ e a soma de Whitney de $\xi$ e $\eta$ é um $(k+l)$-fibrado vetorial sobre $B$.

Exemplo 25. Seja $M^{m}$ uma variedade. Seja $T(M)=\bigcup_{x \in M}\left(\{x\} \times T_{x}(M)\right)$ o conjunto de todos os "vetores tangentes" a $M$, onde $T_{x}(M) \cong \mathbb{R}^{m}$ é o espaço tangente a $M$ em $x$. Então $\tau_{M}=\left(T(M), p, M, \mathbb{R}^{m}\right)$, onde $p(x, v)=x$, é um fibrado vetorial, chamado de fibrado tangente de $M$.

Exemplo 26. Sejam $M^{m} \subset N^{n} \subset \mathbb{R}^{k}$ variedades. Seja $E=\bigcup_{x \in M}(\{x\} \times$ $\left.\left(T_{x}(M)\right)^{\perp}\right)$ o subconjunto de $T(N)$ de todos os "vetores normais" a $M$, onde $T_{x}(M) \oplus\left(T_{x}(M)\right)^{\perp} \cong T_{x}(N)$. Então $\nu(M, N)=\left(E, q, M, \mathbb{R}^{n-m}\right)$, onde $q(x, v)=x$, é um fibrado vetorial, chamado de fibrado normal de $M \mathrm{em}$ $N$. Desta forma, $\tau_{M} \oplus \nu(M, N) \cong\left(\tau_{N}\right)_{\mid M}$. Dizemos que um fibrado $\nu(M) e ́$ um fibrado normal de $M$ se $\tau(M) \oplus \nu(M)$ é trivial. Por exemplo, $\nu\left(M, \mathbb{R}^{k}\right)$ é um fibrado normal de $M$.

Definição 27. Sejam $\xi$ um fibrado vetorial e $M$ uma variedade. Dizemos que $\xi$ é estavelmente trivial, se $\xi \oplus \mathbb{R}^{s} \cong \mathbb{R}^{t}$, para algum $s, t \in \mathbb{N}$. Dizemos que $M$ é (estavelmente) paralelizável, se $\tau_{M}$ é (estavelmente) trivial.

Exemplo 28. A esfera $S^{n}$ é estavelmente paralelizável, para todo $n \geqslant 1$, pois $\tau_{S^{n}} \oplus \mathbb{R}^{1} \cong \tau_{S^{n}} \oplus \nu\left(S^{n}, \mathbb{R}^{n+1}\right) \cong \mathbb{R}^{n+1}$, e é paralelizável se, e só se, $n=1,3,7$. 
O espaço projetivo real $\mathbb{R} P(n)$ é paralelizável se, e só se, $n=1,3,7$. Para maiores detalhes, vide [MiSta-74] (página 47) e [Hu-94] (página 118). Toda 3-variedade fechada orientável é paralelizável (teorema de Stiefel, [Sti-35]). Todo grupo de Lie é paralelizável.

Teorema 29. Sejam $f_{1}, f_{2}: B^{\prime} \rightarrow B$ aplicações contínuas tais que $f_{1} \simeq f_{2}$, com $B^{\prime}$ é paracompacto. Se $\xi$ é um fibrado vetorial sobre $B$, então os pullbacks $f_{1}^{*}(\xi)$ e $f_{2}^{*}(\xi)$ são isomorfos sobre $B^{\prime}$.

Tal resultado pode ser encontrado em [Hu-94] (página 30).

Observação 30. Dizemos que um fibrado $\xi$ é um fibrado esférico, se $F(\xi) \approx S^{a}$, para algum $a \in \mathbb{N}^{*}$. Consideremos um $(k+1)$-fibrado vetorial $\xi=\left(E, p, B, \mathbb{R}^{k+1}\right)$, com $B$ paracompacto e Hausdorff. Então $\xi$ tem grupo estrutural $O(k+1)(S O(k+1))$. Seja $S(E) \subset E$ o subconjunto de todos os vetores unitários de $E$. Então $S(\xi):=\left(S(E), p_{\mid}, B, S^{k}\right)$ é o fibrado esférico associado ao fibrado vetorial $\xi$. Notemos que $S(\xi)$ tem grupo estrutural $O(k+1)(S O(k+1))$. Lembremos que $S O(k+1) \subset O(k+1) \subset G L(k+1) \subset$ $\operatorname{Homeo}\left(\mathbb{R}^{k+1}\right)$, onde $G L(k+1):=G L\left(\mathbb{R}^{k+1}\right)$ é o grupo linear geral em $k+1$ dimensões, $O(k+1)$ é o grupo ortogonal em $k+1$ dimensões (formado pelos isomorfismos de $\mathbb{R}^{k+1}$ em $\mathbb{R}^{k+1}$ que preservam norma, ou equivalentemente formado pelas matrizes ortogonais de ordem $k+1)$ e $S O(k+1)$ é o grupo especial ortogonal em $k+1$ dimensões (formado pelas matrizes ortogonais de ordem $k+1$, com determinante igual a 1 ).

Definição 31. Seja $p: E \rightarrow B$ um fibrado. Uma seção (ou seção transversal) de $p$ é uma aplicação contínua $s: B \rightarrow E$ tal que $p \circ s=1_{B}$. Se $p$ é um fibrado vetorial, dizemos que s é uma seção que não se anula se s(b) é um vetor não nulo, para todo $b \in B$. Ainda, chamamos uma seção de $\tau_{M}$ de seção de $M$ ou de campo de vetores em $M$. 
Observação 32. Todo fibrado vetorial admite uma seção, a saber a seção nula, $b \mapsto \overrightarrow{0}_{b}$.

A seguir, fazemos uma observação sobre classificação de fibrados.

Observação 33. Seja $\xi=\left(E, p, S^{b}, F\right)$ um fibrado sobre $S^{b}$. De modo geral, tais fibrados são classificados, a menos de isomorfismo, por $\pi_{b-1}(\operatorname{Homeo}(F))$. Se, agora, $\xi$ é um $(a+1)$-fibrado vetorial, então quem classifica os fibrados $\xi$ é $\pi_{b-1}(G L(a+1))$ e quem classifica os fibrados $S(\xi)$ é $\pi_{b-1}(O(a+1))$ (vide [Hu-94], página 120). Notemos que $\pi_{b-1}(\operatorname{Homeo}(F))=\pi_{b-1}\left(\operatorname{Homeo}^{+}(F)\right)$, $\pi_{b-1}(G L(a+1))=\pi_{b-1}\left(G L^{+}(a+1)\right)$ e $\pi_{b-1}(O(a+1))=\pi_{b-1}(S O(a+1))$, onde $\mathrm{Homeo}^{+}(F)$ é a componente que contém a identidade e $G L^{+}(a+1)$ é a componente que contém a identidade (formada pelas matrizes invertíveis de ordem $a+1$, com determinante positivo). Analogamente, se $E, F$ são variedades e p é uma aplicação diferenciável, então quem classifica é $\pi_{b-1}(\operatorname{Diff}(F))=$ $\pi_{b-1}\left(\operatorname{Diff}^{+}(F)\right)$.

O resultado abaixo nos diz que, sob determinadas hipóteses, um fibrado é uma fibração.

Teorema 34. Se $p: E \rightarrow B$ é um fibrado, com B paracompacto e Hausdorff, então p é uma fibração.

Tal resultado pode ser encontrado em [Sp-66] (teorema 13, página 96). Desta forma, se $B$ é uma variedade, então todo fibrado $p: E \rightarrow B$ é uma fibração, pois $B$ é em particular um espaço topológico paracompacto e Hausdorff.

Exemplo 35. Sobre aplicações de Hopf e espaços projetivos:

Sejam $S^{1} \subset \mathbb{C}$ e $S^{3} \subset \mathbb{C}^{2}$. Consideremos a ação livre de $S^{1}$ sobre $S^{3}$ dada por $S^{1} \times S^{3} \ni\left(\lambda,\left(z_{1}, z_{2}\right)\right) \mapsto\left(\lambda z_{1}, \lambda z_{2}\right)$. Consideremos o espaço de órbitas, $\frac{S^{3}}{S^{1}}$, 
da ação. Temos que a aplicação quociente $p: S^{3} \rightarrow \frac{S^{3}}{S^{1}} \approx S^{2}$ é uma fibração chamada de aplicação de Hopf, ou fibração de Hopf.

Mais geralmente, seja $S^{2 n+1} \subset \mathbb{C}^{n+1}$ e consideremos a ação livre de $S^{1}$ sobre $S^{2 n+1}$ análoga à anterior. Consideremos o espaço de órbitas, $\mathbb{C} P(n):=\frac{S^{2 n+1}}{S^{1}}$, da ação. Temos que a projeção $p: S^{2 n+1} \rightarrow \mathbb{C} P(n)$ é chamada de aplicação de Hopf, ou fibração de Hopf, ou fibração de Hopf sobre $\mathbb{C} P(n)$. Notemos ainda que $\left(S^{2 n+1}, p, \mathbb{C} P(n), S^{1}\right)$ é um $S^{1}$-fibrado sobre $\mathbb{C} P(n)$. De uma outra forma, se consideramos a relação de equivalência em $S^{2 n+1}$ dada por $\left(z_{1}^{\prime}, \ldots, z_{n+1}^{\prime}\right) \sim\left(z_{1}, \ldots, z_{n+1}\right)$ se, e só se, $z_{i}^{\prime}=\lambda z_{i}$, para todo $i$, para algum $\lambda \in \mathbb{C} \operatorname{com}|\lambda|=1$, temos que $\frac{S^{2 n+1}}{\sim}=\mathbb{C} P(n)$. Com a notação dada, notemos em particular que $\mathbb{C} P(1) \approx S^{2}$.

Sejam agora $S^{3} \subset \mathbb{H}$ e $S^{7} \subset \mathbb{H}^{2}$. Consideremos a ação livre de $S^{3}$ sobre $S^{7}$ dada por $S^{3} \times S^{7} \ni\left(\lambda,\left(z_{1}, z_{2}\right)\right) \mapsto\left(\lambda z_{1}, \lambda z_{2}\right)$. Consideremos o espaço de órbitas, $\frac{S^{7}}{S^{3}}$, da ação. Temos que a aplicação quociente $p: S^{7} \rightarrow \frac{S^{7}}{S^{3}} \approx S^{4}$ é uma fibração chamada de aplicação de Hopf, ou fibração de Hopf.

De modo mais geral, seja $S^{4 n+3} \subset \mathbb{H}^{n+1}$ e consideremos a ação livre de $S^{3}$ sobre $S^{4 n+3}$ da mesma forma que a anterior. Consideremos o espaço de órbitas, $\mathbb{H} P(n):=\frac{S^{4 n+3}}{S^{3}}$, da ação. Temos que a projeção $p: S^{4 n+3} \rightarrow \mathbb{H} P(n)$ é chamada de aplicação de Hopf, ou fibração de Hopf, ou fibração de Hopf sobre $\mathbb{H} P(n)$. Observemos ainda que $\left(S^{4 n+3}, p, \mathbb{H} P(n), S^{3}\right)$ é um $S^{3}$-fibrado sobre $\mathbb{H} P(n)$. De uma outra maneira, se consideramos a relação de equivalência em $S^{4 n+3}$ dada por $\left(z_{1}^{\prime}, \ldots, z_{n+1}^{\prime}\right) \sim\left(z_{1}, \ldots, z_{n+1}\right)$ se, e só se, $z_{i}^{\prime}=\lambda z_{i}$, para todo $i$, para algum $\lambda \in \mathbb{H} \operatorname{com}|\lambda|=1$, obtemos que $\frac{S^{4 n+3}}{\sim}=\mathbb{H} P(n)$. Com a notação dada, observemos que $\mathbb{H} P(1) \approx S^{4}$.

Analogamente, se $S^{0}:=\{ \pm 1\} \subset \mathbb{R}$ e $S^{n} \subset \mathbb{R}^{n+1}$, obtemos $\mathbb{R} P(n):=\frac{S^{n}}{S^{0}}$. Ainda, $p: S^{n} \rightarrow \mathbb{R} P(n)$ é chamada de aplicação de Hopf, ou fibração de Hopf, ou fibração de Hopf sobre $\mathbb{R} P(n)$. Observemos ainda que $p$ é o 
revestimento universal de $\mathbb{R} P(n)$, se $n \geqslant 2$ e $\mathbb{R} P(1) \approx S^{1}$. De um outro modo, se consideramos a relação de equivalência em $S^{n}$ dada por $x^{\prime} \sim x$ se, e só se, $x^{\prime}= \pm x$, obtemos que $\frac{S^{n}}{\sim}=\mathbb{R} P(n)$.

Se $\mathbb{F}=\mathbb{R}$ (respectivamente, $\mathbb{C}, \mathbb{H})$, notemos que $\mathbb{F} P(n)$ é uma $d n$-variedade fechada, conexa, onde $d=\operatorname{dim}_{\mathbb{R}} \mathbb{F}$, chamada de $n$-ésimo espaço projetivo real (respectivamente, complexo, quaterniônico).

Observação 36. Vamos, agora, analisar um pouco melhor a aplicação de Hopf $h: S^{3} \rightarrow S^{2}$ e fazer algumas considerações para serem utilizadas posteriormente. Quando conveniente consideraremos as identificações $\mathbb{C}=\mathbb{R}^{2} \mathrm{e}$ $\mathbb{C}^{2}=\mathbb{R}^{4}$ dadas por $a+b i=(a, b)$ e $(a+b i, c+d i)=(a, b, c, d)$, com $a, b, c, d \in$ $\mathbb{R}$. Considerando $S_{+}^{2}:=\left\{(a+b i, c, 0) \in \mathbb{C} \times \mathbb{R}_{+} \times 0 \mid a^{2}+b^{2}+c^{2}=1\right\} \subset \mathbb{C}^{2}$, $S^{1} \times 0_{\mathbb{C}} \subset \mathbb{C}^{2}$ e $S^{2}=\frac{S_{+}^{2}}{S^{1} \times 0_{\mathbb{C}}}$, obtemos que $h$ pode ser dada por:

$$
h\left(z_{1}, z_{2}\right)= \begin{cases}S^{1} \times 0_{\mathbb{C}} & , \quad z_{2}=0, \\ {\left[\left(\frac{z_{1} \overline{z_{2}}}{\left|z_{2}\right|},\left|z_{2}\right|, 0\right)\right],} & \text { caso contrário. }\end{cases}
$$

Notemos que, desta forma, $h^{-1}\left[\left(0_{\mathbb{C}}, 1,0\right)\right]=0_{\mathbb{C}} \times S^{1} \subset S^{3} \subset \mathbb{C}^{2}$. Considere$\operatorname{mos}$ agora $S_{+}^{2 \prime}:=\left\{(a, 0, b+c i) \in \mathbb{R}_{+} \times 0 \times \mathbb{C} \mid a^{2}+b^{2}+c^{2}=1\right\} \subset S^{3} \subset \mathbb{C}^{2} . \mathrm{Em}$ particular, $\partial S_{+}^{2 \prime}=0_{\mathbb{C}} \times S^{1}$. Seja $h^{\prime}:=h_{\mid S_{+}^{2 \prime}}:\left(S_{+}^{2 \prime}{ }^{\prime} 0_{\mathbb{C}} \times S^{1}\right) \rightarrow\left(S^{2},\left[\left(0_{\mathbb{C}}, 1,0\right)\right]\right)$. Então

$$
h^{\prime}(a, 0, b, c)= \begin{cases}{[(1,0,0,0)]=S^{1} \times 0_{\mathbb{C}}} & , \quad b=0=c, \\ {\left[\left(\frac{a b}{\sqrt{b^{2}+c^{2}}}, \frac{-a c}{\sqrt{b^{2}+c^{2}}}, \sqrt{b^{2}+c^{2}}, 0\right)\right]} & , \text { caso contrário }\end{cases}
$$

é um homeomorfismo relativo. Daí, considerando a aplicação quociente $p r$ : $\left(S_{+}^{2 \prime}, 0_{\mathbb{C}} \times S^{1}\right) \rightarrow\left(\frac{S_{+}^{2 \prime}}{0_{\mathbb{C}} \times S^{1}}, 0_{\mathbb{C}} \times S^{1}\right)$ e identificando $S^{2}=\frac{S_{+}^{2 \prime}}{0_{\mathbb{C}} \times S^{1}}$, obtemos, por [V-94] (proposição 2.5), um homeomorfismo $h^{\prime \prime}: S^{2} \rightarrow S^{2}$ que fatora $h^{\prime}$ conforme o diagrama geométrico abaixo.

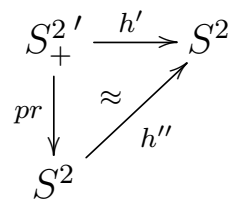


Observação 37. De maneira análoga, vamos analisar um pouco melhor a aplicação de Hopf $p: S^{2 n+1} \rightarrow \mathbb{C} P(n)$ e fazer algumas considerações para serem usadas posteriormente. Quando conveniente consideraremos a identificação $\mathbb{C}^{k}=\mathbb{R}^{2 k}$ dada por $\left(a_{1}+b_{1} i, \ldots, a_{k}+b_{k} i\right)=\left(a_{1}, b_{1}, \ldots, a_{k}, b_{k}\right)$, com $k \geqslant 1$. Analogamente ao que foi feito para $h: S^{3} \rightarrow S^{2}$ (vide a observação 36), seja $p$ dada por:

$$
p\left(z_{1}, \ldots, z_{n+1}\right)= \begin{cases}S^{1} \times 0_{\mathbb{C}} \times \cdots \times 0_{\mathbb{C}} & , z_{2}=\cdots=z_{n+1}=0 \\ {\left[\left(\frac{z_{1} \overline{z_{2}}}{\left|z_{2}\right|},\left|z_{2}\right|, 0, \frac{z_{3} \overline{z_{2}}}{\left|z_{2}\right|}, \ldots, \frac{z_{n+1} \overline{z_{2}}}{\left|z_{2}\right|}\right)\right]} & , z_{2} \neq 0, \\ \vdots & \\ {\left[\left(\frac{z_{1} \overline{z_{n+1}}}{\left|z_{n+1}\right|}, \frac{z_{2} \overline{z_{n+1}}}{\left|z_{n+1}\right|}, \ldots, \frac{z_{n} \overline{z_{n+1}}}{\left|z_{n+1}\right|},\left|z_{n+1}\right|, 0\right)\right], z_{n+1} \neq 0 .}\end{cases}
$$

Notemos que, desta forma, $p^{-1}\left[\left(0_{\mathbb{C}}, 1,0,0_{\mathbb{C}}, \ldots, 0_{\mathbb{C}}\right)\right]=0_{\mathbb{C}} \times S^{1} \times 0_{\mathbb{C}} \times \cdots \times 0_{\mathbb{C}} \subset$ $S^{2 n+1}$. Consideremos agora $S_{+}^{2 \prime}:=\left\{\left(a, 0, b+c i, 0_{\mathbb{C}}, \ldots, 0_{\mathbb{C}}\right) \in \mathbb{R}_{+} \times 0 \times\right.$ $\left.\mathbb{C} \times 0_{\mathbb{C}} \times \cdots \times 0_{\mathbb{C}} \mid a^{2}+b^{2}+c^{2}=1\right\} \subset S^{2 n+1}$. Em particular, $\partial S_{+}^{2 \prime}=$ $0_{\mathbb{C}} \times S^{1} \times 0_{\mathbb{C}} \times \cdots \times 0_{\mathbb{C}}$. Analogamente, obtemos que $p^{\prime}:=p_{\mid S_{+}^{2 \prime}}:\left(S_{+}^{2 \prime}, \partial S_{+}^{2 \prime}\right) \rightarrow$ $\left(S^{2},\left[\left(0_{\mathbb{C}}, 1,0,0_{\mathbb{C}}, \ldots, 0_{C}\right)\right]\right)$ dada por

$p^{\prime}(a, 0, b, c, 0, \ldots, 0)=\left\{\begin{array}{l}{[(1,0, \ldots, 0)]=S^{1} \times 0_{\mathbb{C}} \times \cdots \times 0_{\mathbb{C}}, b=0=c,} \\ {\left[\left(\frac{a b}{\sqrt{b^{2}+c^{2}}}, \frac{-a c}{\sqrt{b^{2}+c^{2}}}, \sqrt{b^{2}+c^{2}}, 0, \ldots, 0\right)\right], \text { caso contrário }}\end{array}\right.$

é um homeomorfismo relativo. Daí, obtemos um homeomorfismo $p^{\prime \prime}: S^{2} \rightarrow$ $S^{2}$ que fatora $p^{\prime}$ conforme o diagrama geométrico abaixo.

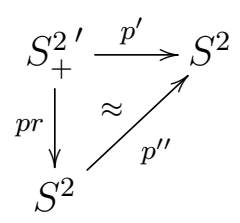

\subsection{Sobre CW-complexos}

Em toda a seção, sejam $X, Y$ espaços topológicos e $y_{0} \in Y$. 
Nesta seção, apresentamos algumas definições e alguns resultados sobre CW-complexos.

Observação 38. Vejamos algumas propriedades:

1) Todo CW-complexo é paracompacto. Vide [FrPi-90] (página 29);

2) Se $Y$ é um CW-complexo, então $\Omega\left(Y, y_{0}\right)$ também o é. Vide [FrPi-90] (página 236);

3) Se $p: X \rightarrow Y$ é uma fibração, com $Y$ sendo um CW-complexo conexo por caminhos e $X$ um CW-complexo, então $p^{-1}(y)$ é um CW-complexo, para todo $y \in Y$. Vide [FrPi-90] (página 237);

4) Se $p: X \rightarrow Y$ é uma fibração, com $Y$ sendo um CW-complexo conexo por caminhos e $p^{-1}(y)$ um CW-complexo, para todo $y \in Y$, então $X$ é um CW-complexo. Vide [FrPi-90] (página 238).

Definição 39. Um $C W$-complexo é dito finito se tem um número finito de células e é dito de tipo finito se cada esqueleto tem um número finito de células.

Observação 40. Um $C W$-complexo é finito se, e só se, é compacto. Ainda, um $C W$-complexo é de tipo finito se, e só se, cada esqueleto é compacto.

Definição 41. Seja $f: X \rightarrow Y$ uma aplicação contínua. Dizemos que $f$ é uma equivalência de homotopia fraca, se $f_{\#}: \pi_{0}(X) \rightarrow \pi_{0}(Y)$ é uma bijeção e $f_{\#}: \pi_{n}(X) \rightarrow \pi_{n}(Y)$ é um isomorfismo, para todo $n \geqslant 1$.

Observação 42. Toda equivalência de homotopia é uma equivalência de homotopia fraca.

Teorema 43. Uma aplicação contínua entre $C W$-complexos é uma equivalência de homotopia fraca se, e somente se, é uma equivalência de homotopia. 
Tal resultado pode ser encontrado em [Sp-66] (corolário 24, página 405).

Teorema 44. Uma equivalência de homotopia fraca induz isomorfismos em homologia (com coeficientes inteiros). Reciprocamente, uma aplicação entre espaços simplesmente conexos que induz isomorfismos em homologia (com coeficientes inteiros) é uma equivalência de homotopia fraca.

Tal resultado pode ser encontrado em [Sp-66] (teorema 25, página 406).

Definição 45. Uma $\mathbf{C W}$-aproximação de um espaço topológico $X$ consiste de um $C W$-complexo $Y$ e de uma equivalência de homotopia fraca $f: Y \rightarrow X$.

Teorema 46. Todo espaço topológico $X$ tem uma $C W$-aproximação. Ainda, duas $C W$-aproximações de um mesmo espaço topológico $X$ têm o mesmo tipo de homotopia.

Tal resultado pode ser encontrado em [Sp-66] (teorema 1, página 412).

Definição 47. Sejam $X_{1}, X_{2}$ espaços topológicos. Dizemos que $X_{1}, X_{2}$ têm o mesmo tipo de homotopia fraca, se existem um espaço topológico $Y$ e equivalências de homotopia fraca $f_{1}: Y \rightarrow X_{1}$ e $f_{2}: Y \rightarrow X_{2}$.

Observação 48. Se $X_{1}, X_{2}$ têm o mesmo tipo de homotopia fraca, então existem $f_{1}: Y \rightarrow X_{1}, f_{2}: Y \rightarrow X_{2}$ equivalências de homotopia fraca. Seja $g: Z \rightarrow Y$ uma $C W$-aproximação de $Y$. Daí, $f_{1} \circ g: Z \rightarrow X_{1}, f_{2} \circ g: Z \rightarrow X_{1}$ são $C W$-aproximações de $X_{1}, X_{2}$, respectivamente, donde, $X_{1}, X_{2}$ podem ser aproximados pelo mesmo $C W$-complexo. Reciprocamente, se $X_{1}, X_{2}$ podem ser aproximados pelo mesmo $C W$-complexo, então $X_{1}, X_{2}$ têm o mesmo tipo de homotopia fraca.

O próximo resultado nos fornece condições suficientes para um CWcomplexo ter o mesmo tipo de homotopia de um CW-complexo de tipo finito. 
Lema 49. Seja $X$ um $C W$-complexo conexo por caminhos tal que $\pi_{n}(X)$ é abeliano finitamente gerado, para todo $n \geqslant 1$. Então $X$ tem o mesmo tipo de homotopia de um $C W$-complexo de tipo finito.

Demonstração. De fato, seja $\pi:=\pi_{1}(X)$. Primeiramente, notemos que, desde que $\pi$ é abeliano finitamente gerado, então, por [P-77] (teorema 2.6, página 423$)$, segue que $\mathbb{Z}[\pi]$ é Noetheriano. Agora, seja $\tilde{X}$ o revestimento universal de $X$. Como $\pi_{n}(\tilde{X}) \cong \pi_{n}(X)$, para todo $n \geqslant 2$, então $\pi_{n}(\tilde{X})$ é finitamente gerado, para todo $n \geqslant 2$. Daí, desde que $\widetilde{X}$ é simplesmente conexo e $\pi_{n}(\tilde{X})$ é finitamente gerado, para todo $n \geqslant 1$, então, por [Se-53] (teorema 1 , capítulo III), $H_{n}(\widetilde{X})$ é finitamente gerado, para todo $n \geqslant 1$. Em particular, $H_{n}(\widetilde{X})$ é finitamente gerado como $\mathbb{Z}[\pi]$-módulo, para todo $n \geqslant 1$. Agora, por [Wa-65] (teoremas A e B), $X$ tem o mesmo tipo de homotopia de um CW-complexo de tipo finito se, e somente se, $\pi$ é finitamente gerado, $\pi$ é finitamente presentado e $H_{n}(\widetilde{X})$ é finitamente gerado como $\mathbb{Z}[\pi]$-módulo, para todo $n \geqslant 1$. Assim, desde que $\pi$ é abeliano finitamente gerado, então tais hipóteses são satisfeitas e portanto $X$ tem o mesmo tipo de homotopia de um CW-complexo de tipo finito.

\subsection{Sobre variedades}

Nesta seção, apresentamos alguns resultados gerais sobre variedades.

Observação 50. Sobre superfícies fechadas, conexas:

Observemos que uma superfície fechada, conexa, $Y$ ou tem grupo fundamental finito e é revestida por $S^{2}$ (que são $S^{2}$ e $\mathbb{R} P(2)$ ), ou é $K(\pi, 1)$ e é revestida por $\mathbb{R}^{2}$ (que são as superfícies fechadas com característica de Euler não positiva). Neste último caso, notemos que $\pi \cong \pi_{1}(Y)$ é infinito. Ainda, se $Y$ é 
$K(\pi, 1)$, segue que $\pi$ é livre de torção e, se $\pi$ é abeliano, então $\pi \cong \mathbb{Z} \oplus \mathbb{Z}$ e $Y \approx T^{2}$.

Observação 51. Sobre 3-variedades fechadas, conexas:

Notemos que uma 3-variedade fechada, conexa, $X$ ou tem grupo fundamental finito e é revestida por $S^{3}$ (que são as formas espaciais esféricas), ou é $K(\pi, 1)$ e é revestida por $\mathbb{R}^{3}$, ou pertence ao conjunto $\left\{S^{1} \times S^{2}, \mathbb{R} P(3) \# \mathbb{R} P(3)\right.$, $\left.S^{1} \tilde{\times} S^{2}, S^{1} \times \mathbb{R} P(2)\right\}$ e é revestida por $\mathbb{R} \times S^{2}$ (vide [Ha-04] e [Tol-74]). Nestes últimos dois casos, observemos que $\pi_{1}(X)$ é infinito. Ainda, por [Ha-04], se $X$ é $K(\pi, 1)$, temos que $\pi \cong \pi_{1}(X)$ é livre de torção e, se $\pi$ é abeliano, então $\pi \cong \mathbb{Z} \oplus \mathbb{Z} \oplus \mathbb{Z}$ e $X \approx T^{3}$.

Lema 52 (Lema da isotopia/Lema da homogeneidade). Sejam Y uma variedade sem bordo, conexa e $y, z \in Y$. Então existe um difeomorfismo $h: Y \rightarrow Y$ isotópico à identidade de $Y$ tal que $h(y)=z$.

Tal resultado pode ser encontrado em [GuPo-74] (página 142).

Lema 53. Sejam Y uma variedade sem bordo, conexa, de dimensão maior do que 1 e $\left\{y_{1}, \ldots, y_{k}\right\},\left\{z_{1}, \ldots, z_{k}\right\} \subset Y$ subconjuntos tais que $y_{i} \neq y_{j}, z_{i} \neq z_{j}$ se $i \neq j$. Então existe um difeomorfismo $h: Y \rightarrow Y$ isotópico à identidade de $Y$ tal que $h\left(y_{l}\right)=z_{l}, l=1, \ldots, k$.

Tal resultado pode ser encontrado em [GuPo-74] (página 143).

Proposição 54. Toda variedade fechada, conexa, de dimensão ímpar tem característica de Euler zero.

Tal resultado segue do teorema dos coeficientes universais e da dualidade de Poincaré. Vide [Ha-02] (página 249).

Proposição 55. Seja $M$ uma variedade fechada, conexa. Então $\chi(M)=0$ se, e somente se, $M$ admite seção que não se anula. 
Tal resultado pode ser encontrado em [Ho-27].

Corolário 56. Toda variedade fechada, conexa, de dimensão ímpar admite seção que não se anula.

O resultado segue dos dois resultados anteriores.

Observação 57. Sobre variedades:

1) Se uma variedade é conexa então ela também é conexa por caminhos, pois é localmente conexa por caminhos.

2) Uma variedade simplesmente conexa é, em particular, orientável, pois os seus laços são todos homotópicos ao laço trivial.

3) Toda variedade é paracompacta, pois é localmente compacta, Hausdorff e satisfaz o segundo axioma de enumerabilidade (vide [Mi-58], página 7).

\subsection{Coincidências}

Em toda a seção, sejam $f, f_{1}, f_{2}: X \rightarrow Y$ aplicações contínuas, onde $X, Y$ são espaços topológicos.

Nesta seção, apresentamos definições e resultados clássicos das teorias de ponto fixo e de coincidência.

Definição 58. Dizemos que $x \in X$ é uma coincidência do par $\left(f_{1}, f_{2}\right)$ de aplicações contínuas, ou uma coincidência entre $f_{1}$ e $f_{2}$, se $f_{1}(x)=f_{2}(x)$. Denotamos o conjunto das coincidências de $\left(f_{1}, f_{2}\right)$ por Coin $\left(f_{1}, f_{2}\right)$.

Observação 59. Notemos que Coin $\left(f_{1}, f_{2}\right)=\left(f_{1}, f_{2}\right)^{-1}(\Delta)$, onde $\Delta:=$ $\{(x, y) \in Y \times Y \mid x=y\} \subset Y \times Y$. De outra forma, notemos que as coincidências entre $f_{1}$ e $f_{2}$ correspondem às interseções entre os gráficos de $f_{1} e$ de $f_{2}$. 
Definição 60. Dizemos que o par $\left(f_{1}, f_{2}\right)$ de aplicações contínuas é deformável a livre de coincidência, ou simplesmente loose, se existem aplicações contínuas $f_{1}^{\prime}, f_{2}^{\prime}: X \rightarrow Y$ tais que $f_{1}^{\prime} \simeq f_{1}, f_{2}^{\prime} \simeq f_{2}$ e $\operatorname{Coin}\left(f_{1}^{\prime}, f_{2}^{\prime}\right)$ é vazio.

$\mathrm{Na}$ teoria de coincidência, estamos interessados, de maneira geral, em estudar o comportamento de $\operatorname{Coin}\left(f_{1}^{\prime}, f_{2}^{\prime}\right)$ para $f_{1}^{\prime} \simeq f_{1}, f_{2}^{\prime} \simeq f_{2}$.

Definição 61. Chamamos $M C\left(f_{1}, f_{2}\right):=\min \left\{\# \operatorname{Coin}\left(f_{1}^{\prime}, f_{2}^{\prime}\right) \mid f_{1}^{\prime} \simeq f_{1}, f_{2}^{\prime} \simeq\right.$ $\left.f_{2}\right\}$ de número mínimo de coincidências de $\left(f_{1}, f_{2}\right)$ e $\operatorname{MCC}\left(f_{1}, f_{2}\right):=$ $\min \left\{\# \pi_{0}\left(\operatorname{Coin}\left(f_{1}^{\prime}, f_{2}^{\prime}\right)\right) \mid f_{1}^{\prime} \simeq f_{1}, f_{2}^{\prime} \simeq f_{2}\right\}$ de número mínimo de componentes de coincidências de $\left(f_{1}, f_{2}\right)$.

Desta forma, notemos que $\left(f_{1}, f_{2}\right)$ é loose se, e só se, $M C\left(f_{1}, f_{2}\right)=0=$ $M C C\left(f_{1}, f_{2}\right)$. Ainda, apesar de $M C\left(f_{1}, f_{2}\right)$ poder ser infinito, supondo $X$ compacto segue que $\operatorname{MCC}\left(f_{1}, f_{2}\right)$ é sempre finito. Os dois resultados abaixo, que podem ser encontrados em [Go-05], mostram, entre outras coisas, algumas situações em que $M C\left(f_{1}, f_{2}\right)$ é finito.

Proposição 62. Sejam $X$ um $C W$ complexo finito e $Y$ uma variedade, onde $\operatorname{dim} X<\operatorname{dim} Y$. Então todo par $\left(f_{1}, f_{2}\right): X \rightarrow Y \times Y$ de aplicações contínuas pode ser deformado a livre de coincidência.

Proposição 63. Sejam $X$ um $C W$ complexo finito e $Y$ uma variedade, onde $\operatorname{dim} X=\operatorname{dim} Y$. Então todo par $\left(f_{1}, f_{2}\right): X \rightarrow Y \times Y$ de aplicações contínuas pode ser deformado a um par $\left(f_{1}^{\prime}, f_{2}^{\prime}\right)$ tal que Coin $\left(f_{1}^{\prime}, f_{2}^{\prime}\right)$ é finito.

O próximo resultado nos diz que, quando $Y$ é uma variedade sem bordo, para calcular $M C\left(f_{1}, f_{2}\right)$, basta deformar apenas uma das aplicações. Tal resultado pode ser encontrado em [Go-05] e pode ser encontrado, numa versão mais forte, em [Broo-72]. 
Proposição 64. Sejam $X$ um espaço topológico e $Y$ uma variedade sem bordo. Então $M C\left(f_{1}, f_{2}\right)=\min \left\{\# \operatorname{Coin}\left(f_{1}^{\prime}, f_{2}\right) \mid f_{1}^{\prime} \simeq f_{1}\right\}$.

Observação 65. Sobre pontos fixos:

Seja $X=Y$. Dizemos que $x \in X$ é um ponto fixo de $f$, se $f(x)=x$. Na teoria de ponto fixo, entre outras coisas, estamos interessados no número mínimo de pontos fixos de $f$, isto é, $\min \left\{\# F i x\left(f^{\prime}\right): f^{\prime} \simeq f\right\}$, onde Fix $\left(f^{\prime}\right):=\left\{x \in X \mid f^{\prime}(x)=x\right\}$. Ainda, queremos determinar quando $f$ é deformável a livre de ponto fixo, isto é, quando existe $f^{\prime} \simeq f$ tal que $F i x\left(f^{\prime}\right)=\emptyset$. Por [Broo-72], sendo $X=Y$ uma variedade sem bordo, tais questões são equivalentes, na teoria de coincidência, às respectivas questões para o par $\left(1_{X}, f\right)$. Em particular, $M C\left(1_{X}, f\right)=\min \left\{\# F i x\left(f^{\prime}\right): f^{\prime} \simeq f\right\}=$ : $M F(f)$.

Observação 66. Sobre raízes:

Sejam $X, Y$ não necessariamente iguais e $y_{0} \in Y$. Dizemos que $x \in$ $X$ é uma raiz de $f$, se $f(x)=y_{0}$. Na teoria de raiz, entre outras coisas, estamos interessados no número mínimo de raízes de $f$, isto é, $\min \left\{\# f^{\prime-1}\left(y_{0}\right): f^{\prime} \simeq f\right\}$ e queremos determinar quando $f$ é deformável a livre de raiz, ou seja, quando existe $f^{\prime} \simeq f$ tal que $f^{\prime-1}\left(y_{0}\right)=\emptyset$. Por [Broo-72], sendo $Y$ uma variedade sem bordo, tais questões são equivalentes, na teoria de coincidência, às respectivas questões para o par $\left(y_{0}, f\right)$. Em particular, $M C\left(y_{0}, f\right)=\min \left\{\# f^{\prime-1}\left(y_{0}\right): f^{\prime} \simeq f\right\}$.

Observação 67. Sobre auto-coincidências:

Sejam $X, Y$ não necessariamente iguais. Para todo $x \in X$, notemos que $x$ é uma coincidência do par $(f, f)$. Na teoria de auto-coincidência, estamos interessados, por exemplo, no número mínimo de auto-coincidências de $f$, ou seja, $M C(f, f):=\min \left\{\# \operatorname{Coin}\left(f^{\prime}, f^{\prime \prime}\right): f^{\prime} \simeq f \simeq f^{\prime \prime}\right\}$. Ainda, 
queremos saber quando $f$ é deformável a livre de auto-coincidência, isto é, quando $(f, f)$ é deformável a livre de coincidência.

Um avanço significativo na teoria de coincidência se deu entre 1923 e 1927, quando Lefschetz ([L-23],[L-25],[L-26],[L-27]) deu condições suficientes para a existência de coincidência, para variedades orientáveis fechadas de mesma dimensão. Ele definiu um índice $I_{f_{1}, f_{2}} \in \mathbb{Z}$ que se anula quando o par $\left(f_{1}, f_{2}\right)$ de aplicações contínuas é deformável a livre de coincidência e mostrou que tal índice é igual à soma alternada de traços, $L\left(f_{1}, f_{2}\right) \in \mathbb{Z}$, de certos homomorfismos. Em particular, se $L\left(f_{1}, f_{2}\right) \neq 0$, então $\operatorname{Coin}\left(f_{1}^{\prime}, f_{2}^{\prime}\right) \neq \emptyset$, para toda $f_{i}^{\prime} \simeq f_{i}, i=1,2$. Abaixo, fazemos uma breve exposição do assunto. Para maiores detalhes, vide [V-94] (capítulo 7).

Definição 68. Sejam $X, Y$ n-variedades orientáveis fechadas. Seja $W \subset_{a b}$ $X$ e sejam $f_{1}, f_{2}: W \rightarrow Y$ aplicações contínuas tais que $C:=\operatorname{Coin}\left(f_{1}, f_{2}\right)$ é um compacto de $W$. Então existe $V \subset_{a b} X$, com $C \subset V \subset \bar{V} \subset W$. Defina o índice de coincidência do par $\left(f_{1}, f_{2}\right)$ sobre $W$ como sendo o inteiro $I_{f_{1}, f_{2}}^{W}$ dado pela imagem da classe fundamental $z \in H_{n}(X ; \mathbb{Z})$ sob a composição $\mathbb{Z} \cong H_{n}(X) \rightarrow H_{n}(X, X-V) \cong H_{n}(W, W-V) \stackrel{\left(f_{1}, f_{2}\right)_{*}}{\rightarrow} H_{n}(Y \times Y, Y \times Y \backslash \Delta) \cong \mathbb{Z}$

Notemos que, a princípio, não é possível definir tal índice, da mesma forma, quando as dimensões das variedades são diferentes.

Observação 69. Tal índice independe de $V$ e tem várias propriedades, entre elas: se $I_{f_{1}, f_{2}}^{W} \neq 0$, então $\left(f_{1}, f_{2}\right)$ tem coincidência em $W$; se $f_{i}^{\prime} \simeq f_{i}, i=1,2$, então, sob certas hipóteses, $I_{f_{1}^{\prime}, f_{2}^{\prime}}^{W}=I_{f_{1}, f_{2}}^{W}$; e o grau local de $f: X \rightarrow X$ num ponto fixo isolado é o indice de ponto fixo local $I_{f}^{W}:=I_{f, 1_{X}}^{W}$. Ainda, dizemos que $I_{f_{1}, f_{2}}:=I_{f_{1}, f_{2}}^{X}$ é o índice de coincidência de $\left(f_{1}, f_{2}\right)$ e $I_{f}:=I_{f}^{X}$ é o índice de ponto fixo de $f$. 
Observação 70. Seja $f: X \rightarrow X$ uma aplicação contínua. Sendo $X$ um complexo finito, temos uma definição de indice de ponto fixo (vide [Ji-83], capítulo 1) que estende a anterior para ponto fixo.

Definição 71. Sejam X,Y n-variedades orientáveis fechadas. Definimos o número de Lefschetz de $(f, g)$ como sendo $L(f, g):=\sum_{q}(-1)^{q} \operatorname{tr} \Theta_{q} \in \mathbb{Q}$, onde $\Theta_{q}=D_{X} \circ g^{*} \circ D_{Y}^{-1} \circ f_{*}: H_{q}(X ; \mathbb{Q}) \rightarrow H_{q}(X ; \mathbb{Q})$, onde $D_{X}, D_{Y}$ são dados pela dualidade de Poincaré.

Observação 72. Usando $\widehat{\Theta}_{q}=D_{Y}^{-1} \circ f_{*} \circ D_{X} \circ g^{*}$, analogamente, definimos $\widehat{L}(f, g)$, também chamado de número de Lefschetz, o qual é igual a $(-1)^{n} L(f, g)$. Observamos, ainda, que $L(f, g)=(-1)^{n} L(g, f)$. Além disto, claramente, o número de Lefschetz é um invariante homotópico.

Observação 73. Seja $f: X \rightarrow X$ uma aplicação contínua. Definimos o número de Lefschetz de $f$ como sendo $L(f):=\sum_{q}(-1)^{q} \operatorname{tr}\left(f_{*}\right)_{q} \in \mathbb{Q}$, onde $\left(f_{*}\right)_{q}: H_{q}(X ; \mathbb{Q}) \rightarrow H_{q}(X ; \mathbb{Q})$. Claramente, o número de Lefschetz é um invariante homotópico. Ainda, notemos que a característica de Euler de $X$ é tal que $\chi(X)=L\left(1_{X}\right)$. Finalmente, observemos que, se X é uma variedade fechada orientável, então $L(f)=L\left(f, 1_{X}\right)$.

Teorema 74 (Teorema de coincidência de Lefschetz). Sejam $f_{1}, f_{2}: X \rightarrow Y$ aplicações contínuas, com $X, Y$ variedades orientáveis fechadas de mesma dimensão. Então o indice de coincidência $I_{f_{1}, f_{2}} \in \mathbb{Z}$ é igual ao número de Lefschetz $L\left(f_{1}, f_{2}\right)$.

Após este resultado, em 1929, Hopf ([Ho-29]) provou um resultado similar para ponto fixo (conhecido como Teorema de ponto fixo de Lefschetz-Hopf), quando os espaços são complexos finitos. Um pouco antes, em 1927, sendo $X$ um complexo finito, Nielsen $([\mathrm{N}-27])$ deu um limitante inferior, $N(f)$, para 
o número mínimo de pontos fixos, $M F(f)$, de $f: X \rightarrow X$ e conjecturou que $M F(f)$ seria igual a $N(f)$, para toda aplicação contínua $f$, sendo $X$ uma superfície compacta (já sabendo que isto era verdade para superfícies compactas com $\chi(X) \geqslant 0)$. Entre 1941 e 1942, Wecken ([We-41],[We-412],[We-42]) mostrou tal igualdade para vários complexos finitos e, em particular, para $n$-variedades compactas, com $n \geqslant 3$. Abaixo, fazemos uma breve exposição do assunto. Para maiores detalhes, vide [Brow-93] e [Go-05].

Definição 75. Sejam $x, y \in \operatorname{Coin}\left(f_{1}, f_{2}\right) \subset X$. Dizemos que $x, y$ são Nielsen equivalentes se existe $\sigma \in X^{I}$ tal que $\sigma(0)=x, \sigma(1)=y e$ $f_{1} \circ \sigma \simeq_{\partial I} f_{2} \circ \sigma$. Uma classe de equivalência é chamada de classe de Nielsen de $\left(f_{1}, f_{2}\right)$.

Observação 76. Da mesma forma, $x, y \in F i x(f) \subset X$ são Nielsen equivalentes se existe $\sigma \in X^{I}$ tal que $\sigma(0)=x, \sigma(1)=y$ e $f \circ \sigma \simeq_{\partial I} \sigma$.

Definição 77. Sejam $X, Y$ variedades orientáveis fechadas de mesma dimensão. Dizemos uma classe de Nielsen, $F$, de $\left(f_{1}, f_{2}\right)$ é essencial se $I_{f_{1}, f_{2}}^{F} \neq 0$ e chamamos o número de classes essenciais de $\left(f_{1}, f_{2}\right)$ de número de Nielsen de $\left(f_{1}, f_{2}\right)$ e o denotamos por $N\left(f_{1}, f_{2}\right)$.

Observação 78. Temos que $N\left(f_{1}, f_{2}\right)$ é finito, $N\left(f_{1}, f_{2}\right)=N\left(f_{2}, f_{1}\right)$ e $N\left(f_{1}, f_{2}\right)$ depende somente das classes de homotopia de $f_{1}$ e de $f_{2}$, ou seja, é um invariante homotópico.

Observação 79. Seja $f: X \rightarrow X$ uma aplicação contínua. Sendo $X$ um complexo finito, temos uma definição de número de Nielsen de $f, N(f)$, que é análoga à definição acima. Além disto, $N(f)$ é finito e depende apenas da classe de homotopia de $f$.

Teorema 80. Sejam $X$ um complexo finito e $f: X \rightarrow X$ uma aplicação contínua. Então $N(f) \leqslant M F(f)$. 
Teorema 81 (Teorema de Wecken). Se X é uma n-variedade compacta, com $n \geqslant 3$, então $M F(f)=N(f)$, para toda aplicação contínua $f: X \rightarrow X$.

Por causa deste resultado, dizemos que um espaço topológico $X$ é de Wecken se $M F(f)=N(f)$, para toda aplicação contínua $f: X \rightarrow X$. Em 1984-5, Jiang ([Ji-84],[Ji-85]) exibiu um contra-exemplo para a conjectura de Nielsen e provou que uma superfície compacta $X$ é de Wecken se, e somente se, $\chi(X) \geqslant 0$, que era exatamente onde Nielsen sabia da validade de sua conjectura. Um pouco antes, em 1955, Schirmer ([Sc-55]), em sua tese, mostrou que, se $X, Y$ são $n$-variedades orientáveis fechadas, com $n \geqslant 3$, então $M C\left(f_{1}, f_{2}\right)=N\left(f_{1}, f_{2}\right)$, para todo par $\left(f_{1}, f_{2}\right)$ de aplicações contínuas.

Referente à teoria de ponto fixo, em 1936, Reidemeister ([Re-36]) definiu um elemento, chamado de traço de Reidemeister, no quociente de $\mathbb{Z}[\pi]$ por uma certa relação (que resulta numa soma de $\mathbb{Z}$ 's indexada no conjunto das classes de Reidemeister, cuja definição veremos a seguir), onde $\pi=\pi_{1}(X)$. O interessante é que todo coeficiente não nulo no traço de Reidemeister corresponde a exatamente uma classe de Nielsen essencial (e vice-versa) cujo índice é o valor do coeficiente. Para maiores detalhes, vide [Ji-83].

Vamos, agora, discutir uma importante ferramenta no estudo de coincidência.

Definição 82. Seja $x_{0} \in X$ tal que $f_{1}\left(x_{0}\right)=f_{2}\left(x_{0}\right)=y_{0}$. Sejam $\alpha, \beta \in$ $\pi_{1}\left(Y, y_{0}\right)$. Dizemos que $\alpha, \beta$ são Reidemeister equivalentes se existe $\theta \in$ $\pi_{1}\left(X, x_{0}\right)$ tal que $\beta=f_{1 \#}(\theta)^{-1} \cdot \alpha \cdot f_{2 \#}(\theta)$. Uma classe de equivalência é chamada de classe de Reidemeister de $\left(f_{1}, f_{2}\right)$. O número de tais classes é chamado de número de Reidemeister de $\left(f_{1}, f_{2}\right)$ e é denotado por $R\left(f_{1}, f_{2}\right)$.

Observação 83. Temos que $R\left(f_{1}, f_{2}\right)$ pode ser infinito, $R\left(f_{1}, f_{2}\right)=R\left(f_{2}, f_{1}\right)$ 
e $R\left(f_{1}, f_{2}\right)$ é um invariante homotópico. Ainda, existe uma injeção do conjunto das classes de Nielsen no conjunto das classes de Reidemeister e, em particular, $N\left(f_{1}, f_{2}\right) \leqslant R\left(f_{1}, f_{2}\right)$. Se $f: X \rightarrow X$ é uma aplicação contínua, então o número de Reidemeister de $f$ é dado por $R(f):=R\left(f, 1_{X}\right)$.

Para uma discussão sobre classes $(H, G)$-relacionadas e classes de Nielsen geometricamente essenciais, vide [Go-05]. Um dos interesses no número de Reidemeister é que, sob certas hipóteses sobre os espaços (por exemplo, sendo $X, Y$ variedades fechadas de mesma dimensão, com $Y$ um grupo de Lie ou um $H$-espaço), segue que $N\left(f_{1}, f_{2}\right)=0$ ou $N\left(f_{1}, f_{2}\right)=R\left(f_{1}, f_{2}\right)$ (igual a $\left.\left[\pi_{1}(Y):\left(f_{1 \#}-f_{2 \#}\right)\left(\pi_{1}(X)\right)\right]\right)$ e todas as classes de Nielsen têm o mesmo índice. Para maiores detalhes, vide [Go-05].

No caso especial de ponto fixo, para uma definição alternativa de número de Reidemeister, com classes de levantamento e classes $H$-relacionadas, vide [Ji-83].

\subsection{Topologia diferencial}

Em toda a seção, a princípio, uma variedade é uma variedade sem bordo.

Definição 84. Sejam $X, Y$ variedades e $f: X \rightarrow Y$ uma aplicação diferenciável. Dizemos que $x \in X$ é um ponto regular de $f$ se $d f_{x}: T_{x}(X) \rightarrow$ $T_{f(x)} Y$ é sobrejetora. Um ponto que não é regular é chamado de crítico (ou singular) de $f$. Dizemos que $y \in Y$ é um valor regular de $f$ se, para cada $x \in X$ tal que $y=f(x)$, temos que $d f_{x}$ é sobrejetora.

Teorema 85 (Teorema da imagem inversa regular). Sejam $X^{m}, Y^{n}$ variedades e $f: X \rightarrow Y$ uma aplicação diferenciável. Então, para todo valor regular $y$ de $f$, a imagem inversa $f^{-1}(y)=: W$ é uma subvariedade de $X$ de 
codimensão $n$ (isto é, de dimensão $m-n$ ). Ainda, $T_{x} W \cong \operatorname{ker}\left(d f_{x}\right)$, para todo $x \in W$.

Tal resultado pode ser encontrado em [Sha-11] (página 89).

Definição 86. Sejam $X, Y$ variedades e $f: X \rightarrow Y$ uma aplicação diferenciável. Seja $Z$ uma subvariedade de $Y$. Dizemos que $f$ é transversal (ou transversa) a $Z$ (e escrevemos $f \pitchfork Z$ ) se, para cada $x \in X$ tal que $y=f(x) \in Z$, temos que $\operatorname{Im}\left(d f_{x}\right)+T_{y}(Z)=T_{y}(Y)$.

Observação 87. Seja $Z=\{z\}$ um conjunto unitário. Se $x \in X$ satisfaz a condição acima, então $d f_{x}$ é sobrejetora (isto é, $f$ é uma submersão em $x$ ). Ainda, isto é verdade para cada $x \in f^{-1}(z)$ se, e somente se, z é um valor regular de $f$. Desta forma, podemos ver a noção de transversalidade como sendo uma generalização da noção de regularidade.

Teorema 88 (Teorema da imagem inversa transversal). Sejam $X, Y$ variedades e $f: X \rightarrow Y$ uma aplicação diferenciável. Seja $Z \subset Y$ uma subvariedade de codimensão $k$, com $Z$ e $Y$ sem bordo. Suponha que $f \pitchfork Z$ e $\partial f \pitchfork Z$, onde $\partial f:=f_{\mid \partial X}$. Então a imagem inversa $f^{-1}(Z)=: W$ é uma subvariedade de $X$ de codimensão $k$ tal que $\partial W=\partial X \cap W$. Ainda, $T_{x}(W)=\left(d f_{x}\right)^{-1}\left(T_{f(x)}(Z)\right)$, para todo $x \in W$.

Tal resultado pode ser encontrado em [Sha-11] (página 94).

Definição 89. Sejam $f, g: X \rightarrow Y$ aplicações contínuas, onde $(Y, d)$ é um espaço métrico. Seja $\delta: X \rightarrow] 0, \infty[$ uma função contínua positiva. Dizemos que g é uma $\delta$-aproximação de $f$ se $d(f(x), g(x))<\delta(x)$, para todo $x \in X$. Dizemos que g é uma aproximação de $f$ se $g$ é uma $\delta$-aproximação de $f$, para alguma função contínua positiva $\delta$. 
Teorema 90 (Teorema do mergulho de Whitney). Toda variedade $Y^{n}$ é uma subvariedade de $\mathbb{R}^{2 n+1}$ como um subconjunto fechado em $\mathbb{R}^{2 n+1}$.

Tal resultado pode ser encontrado em [Mi-58] (página 10). A partir deste resultado, podemos considerar a métrica induzida em $Y$ de $\mathbb{R}^{2 n+1}$. Em particular, podemos considerar aproximações de aplicações contínuas de $X$ em $Y$.

Teorema 91. Sejam $X, Y$ variedades e $C \subset X$ um subconjunto fechado em $X$. Seja $f: X \rightarrow Y$ uma aplicação diferenciável. Seja $Z \subset Y$ uma subvariedade de codimensão $k$ que é um subconjunto fechado em $Y$. Suponha que $f$ é transversal a $Z$ em $C \cap f^{-1}(Z)$. Dada $\left.\delta: X \rightarrow\right] 0, \infty[$ uma função contínua positiva, existe uma aplicação diferenciável $g: X \rightarrow Y$ tal que $g$ é uma $\delta$-aproximação de $f, g$ é transversal a $Z$ e $g_{\mid C}=f_{\mid C}$.

Tal resultado pode ser encontrado em [Mi-58] (página 10).

Lema 92. Sejam $X, Y$ variedades e $C \subset X$ um subconjunto fechado em $X$. Seja $f: X \rightarrow Y$ uma aplicação contínua que é diferenciável em $C$. Dada $\delta: X \rightarrow] 0, \infty[$ uma função contínua positiva, existe uma aplicação diferenciável $g: X \rightarrow Y$ tal que g é uma $\delta$-aproximação de $f$ e $g_{\mid C}=f_{\mid C}$.

Tal resultado pode ser encontrado em [Mi-58] (página 26).

Lema 93. Sejam $X, Y$ variedades. Seja $f: X \rightarrow Y$ uma aplicação contínua. Dada $\epsilon: X \rightarrow] 0, \infty[$ uma função contínua positiva, existe $\delta: X \rightarrow] 0, \infty[$ uma função contínua positiva tal que se $g: X \rightarrow Y$ é uma $\delta$-aproximação de $f$, então existe uma homotopia $H: f \simeq g$, com $H_{t}(x)=f(x)$ para todo $x \in X$ tal que $g(x)=f(x)$, e sendo $H_{t}$ uma $\epsilon$-aproximação de $f$, para todo $t \in I$.

Tal resultado pode ser encontrado em [Mi-58] (página 26). 
Exemplo 94. Sejam $X, Y$ variedades e $f_{1}, f_{2}: X \rightarrow Y$ aplicações contínuas. Então $\left(f_{1}, f_{2}\right): X \rightarrow Y \times Y$ é uma aplicação contínua. Pelo lema 92, existe uma aplicação diferenciável $\left(f_{1}^{\prime}, f_{2}^{\prime}\right): X \rightarrow Y \times Y$ que é uma aproximação de $\left(f_{1}, f_{2}\right)$. Consideremos a diagonal $\Delta \subset Y \times Y$. Notemos que $\Delta \subset Y \times Y$ é uma subvariedade de codimensão $n$ que é um subconjunto fechado em $Y \times Y$. Pelo teorema 91, existe uma aplicação diferenciável $\left(f_{1}^{\prime \prime}, f_{2}^{\prime \prime}\right): X \rightarrow Y \times Y$ que é uma aproximação de $\left(f_{1}^{\prime}, f_{2}^{\prime}\right)$ e é transversal $a \Delta$. Finalmente, pelo lema 93, desde que $\left(f_{1}^{\prime \prime}, f_{2}^{\prime \prime}\right)$ é uma aproximação de $\left(f_{1}, f_{2}\right)$, então $\left(f_{1}^{\prime \prime}, f_{2}^{\prime \prime}\right) \simeq\left(f_{1}, f_{2}\right)$. Desta forma, aproximamos $\left(f_{1}, f_{2}\right)$ por uma aplicação diferenciável (e homotópica a ela) que é transversal a $\Delta$.

\subsection{Bordismo}

Em toda a seção, sejam $X, Y, Z$ espaços topológicos.

Nesta seção, apresentamos conceitos da teoria de bordismo normal, além de identificações com outras teorias de bordismo.

Definição 95. Um fibrado virtual, $\varphi$, sobre $X$ é um par ordenado de fibrados vetoriais, $\left(\varphi_{+}, \varphi_{-}\right)$, sobre $X$. Escrevemos $\varphi:=\varphi_{+}-\varphi_{-}$. Ainda, dizemos que um fibrado virtual é trivial se ele é formado por fibrados vetoriais triviais.

Observação 96. Se $\varphi=\varphi_{+}-\varphi_{-}$é um fibrado virtual sobre $X$, então $-\varphi:=\varphi_{-}-\varphi_{+}$também é um fibrado virtual sobre $X$. Se $g: Z \rightarrow X$ é uma aplicação contínua, definimos o pullback de $\varphi$ por $g$ como sendo o fibrado virtual sobre $Z$ dado por $g^{*}(\varphi):=g^{*}\left(\varphi_{+}\right)-g^{*}\left(\varphi_{-}\right)$.

Definição 97. Sejam $f, f^{\prime}: \xi^{k} \rightarrow \eta^{k}$ isomorfismos de fibrados vetoriais sobre B. Dizemos que $f$ e $f^{\prime}$ são isotópicos (sobre B), se existe uma homotopia 
$H: E(\xi) \times I \rightarrow E(\eta)$ tal que $H_{0}=f, H_{1}=f^{\prime}$ e $H_{t}: \xi \rightarrow \eta$ é um isomorfismo de fibrados vetoriais sobre $B$, para todo $t \in I$, ou seja, $H_{t}: E(\xi) \rightarrow E(\eta)$ é um homeomorfismo que preserva fibra sobre $B$ e $H_{t \mid}: F(\xi) \rightarrow F(\eta)$ é um isomorfismo de espaços vetoriais, para todo $t \in I$. Escrevemos $f \simeq f^{\prime}$.

Definição 98. Sejam $\xi, \eta$ fibrados vetoriais sobre B. Consideremos $\mathcal{C}_{\xi, \eta}:=$ $\left\{f: \xi \oplus \mathbb{R}^{s} \rightarrow \eta \oplus \mathbb{R}^{t}\right.$ isomorfismo de fibrados vetoriais $\left.\mid s, t \in \mathbb{N}\right\}$. Denotemos um isomorfismo, $f$, de $k$-fibrados vetoriais por $f_{k}$. Dados $f_{k}, f_{l}^{\prime} \in \mathcal{C}_{\xi, \eta}$, dizemos que $f_{k}$ e $f_{l}^{\prime}$ são equivalentes:

1. se $f_{k} \times 1_{\mathbb{R}^{r}} \simeq f_{l}^{\prime} \times 1_{\mathbb{R}^{r}}$, quando $k=l$, para algum $r$;

2. se $f_{k} \times 1_{\mathbb{R}^{r}} \simeq f_{l}^{\prime} \times 1_{\mathbb{R}^{k-l}} \times 1_{\mathbb{R}^{r}}$, quando $k>l$, para algum $r$;

3. se $f_{k} \times 1_{\mathbb{R}^{l-k}} \times 1_{\mathbb{R}^{r}} \simeq f_{l}^{\prime} \times 1_{\mathbb{R}^{r}}$, quando $k<l$, para algum $r$.

Denotamos a classe de equivalência de $f \in \mathcal{C}_{\xi, \eta}$ por $f: \xi \rightarrow \eta$ e chamamos uma tal classe de equivalência de um isomorfismo estável de fibrados vetoriais sobre $B$.

Observação 99. Se $f$ é um isomorfismo estável, então $-f$ denota o isomorfismo estável dado por $f \times\left(-1_{\mathbb{R}^{1}}\right): \xi \oplus \mathbb{R}^{s} \oplus \mathbb{R}^{1} \rightarrow \eta \oplus \mathbb{R}^{t} \oplus \mathbb{R}^{1}$, para algum $s, t \in \mathbb{N}$.

Definição 100. Sejam $X$ um espaço topológico e $\varphi$ um fibrado virtual sobre $X$. Uma $\varphi$-variedade compacta (fechada) singular em $X$ de dimensão $m$ é uma tripla $(M, g, \bar{g})$, em que:

- M é uma variedade compacta (fechada) de dimensão $m$,

- g:M $\rightarrow X$ é uma aplicação contínua,

- $\bar{g}: \tau_{M} \oplus g^{*}\left(\varphi_{+}\right) \rightarrow g^{*}\left(\varphi_{-}\right)$é um isomorfismo estável de fibrados vetoriais sobre $M$. 
Observação 101. Notemos que se $\mathcal{M}:=(M, g, \bar{g}), \mathcal{N}:=(N, h, \bar{h})$ são $\varphi$ variedades singulares em $X$ de dimensão $m$, então $\mathcal{M} \cup \mathcal{N}:=(M \dot{\cup} N, g \dot{\cup}$ $h, \bar{g} \cup \bar{h})$ e $-\mathcal{M}:=(M, g,-\bar{g})$ também o são. Se $f: X \rightarrow Y$ é uma aplicação contínua e $\varphi=f^{*}(\psi)$, para algum fibrado virtual $\psi$ sobre $Y$, podemos considerar a $\psi$-variedade singular em $Y$ de dimensão $m$ dada por $f^{*}(\mathcal{M}):=(M, f \circ g, \bar{g})$. Notemos ainda que $\mathcal{O}:=(\emptyset, \emptyset, \emptyset)$ é uma $\varphi$-variedade fechada singular em $X$ de dimensão $m$, independentemente de $\varphi, X$ e m.

Definição 102. Sejam $\mathcal{M}:=(M, g, \bar{g}), \mathcal{N}:=(N, h, \bar{h}) \varphi$-variedades fechadas singulares em $X$ de dimensão $m$. Dizemos que $\mathcal{M}$ e $\mathcal{N}$ são bordantes (e escrevemos $\mathcal{M} \sim \mathcal{N})$, se existe $(B, b, \bar{b})$, uma $\varphi$-variedade compacta singular em $X$ de dimensão $m+1$, tal que $\partial B=M \dot{\cup} N, b_{\mid \partial B}=g \dot{\cup} h e \bar{b}_{\mid \partial B}=\bar{g} \dot{\cup}-\bar{h}$ (isto é, via campos de vetores que apontam pra dentro de $B$ ao longo de $M$ e pra fora de $B$ ao longo de $N$ - o que diz como $\tau_{M}\left(\tau_{N}\right)$ se inclui em $\left.\tau_{B \mid M} \cong \tau_{M} \oplus \mathbb{R}^{1}\left(\tau_{B \mid N} \cong \tau_{N} \oplus \mathbb{R}^{1}\right)\right)$. Ainda, dizemos que $\mathcal{M}$ é nula bordante, se $\mathcal{M} \sim \mathcal{O}$.

Observação 103. Temos que $\sim$ é uma relação de equivalência. Denotamos a classe de equivalência de $\mathcal{M}$ por $[\mathcal{M}]$ e o conjunto de classes de equivalência de $\varphi$-variedades fechadas singulares em $X$ de dimensão $m$ por $\Omega_{m}(X ; \varphi)$. Ainda, notemos que $\mathcal{M} \sim \mathcal{N}$ se, e só se, $\mathcal{M} \cup(-\mathcal{N}) \sim \mathcal{O}$.

Definição 104. Definindo $[\mathcal{M}]+[\mathcal{N}]:=[\mathcal{M} \cup \mathcal{N}]$, temos que $\left(\Omega_{m}(X ; \varphi),+\right)$ é um grupo abeliano, chamado de m-ésimo grupo de bordismo normal de $X$ com coeficientes em $\varphi$.

Observação 105. Seja $\varphi=\varphi_{+}-\varphi_{-}$um fibrado virtual sobre $X$. Notemos que $\Omega_{m}(X ; \varphi) \cong \Omega_{m}\left(X ; \varphi_{+} \oplus \mathbb{R}^{t}-\varphi_{-} \oplus \mathbb{R}^{s}\right)$, para todo $s, t \in \mathbb{N}$. Em particular, se $\varphi_{+}$é estavelmente trivial, podemos considerar $\varphi_{+}$como sendo trivial 
e denotamos $\varphi=-\varphi_{-}$. Da mesma forma, se $\varphi_{-}$é estavelmente trivial, podemos considerar $\varphi_{-}$como sendo trivial e escrevemos $\varphi=\varphi_{+}$. Se ambos são estavelmente triviais, podemos considerar $\varphi$ como sendo trivial e, sem perda de generalidade, denotamos $\varphi=\mathbb{R}$.

Observação 106. Se $f: X \rightarrow Y$ é uma aplicação contínua e $\psi$ é um fibrado virtual sobre $Y$, podemos considerar o homomorfismo induzido $f_{*}$ : $\Omega_{m}\left(X ; f^{*}(\psi)\right) \rightarrow \Omega_{m}(Y ; \psi)$ dado por $f_{*}([\mathcal{M}])=\left[f^{*}(\mathcal{M})\right]$.

Definição 107. Temos que $\Omega_{m}^{f r}(X):=\Omega_{m}(X ; \mathbb{R})$ é chamado de m-ésimo grupo de bordismo "framed" de $X$ e $\Omega_{m}^{f r}:=\Omega_{m}^{f r}\left(y_{0}\right)$ é chamado simplesmente de m-ésimo grupo de bordismo "framed", onde yo é um ponto.

Observação 108. Sobre o isomorfismo de Pontryagin-Thom:

Seja $m \geqslant n$. Seja $C^{m-n} \subset S^{m}$ uma subvariedade de $S^{m}$ de codimensão n. Seja $g^{\prime}: \nu\left(C, S^{m}\right) \rightarrow \mathbb{R}^{n}$ um isomorfismo de fibrados vetoriais sobre $C$, ou seja, uma trivialização do fibrado normal $\nu\left(C, S^{m}\right)$. Considerando os pares $\left(C, g^{\prime}\right)$ e uma relação de bordismo análoga à relação anterior, obtemos o grupo de bordismo orientado $\Omega_{m-n}$. Agora, seja $\overline{g^{\prime}}: \nu\left(C, S^{m}\right) \rightarrow \mathbb{R}^{n} u m$ isomorfismo estável de fibrados vetoriais sobre $C$, ou melhor, a trivialização estável do fibrado normal $\nu\left(C, S^{m}\right)$. Considerando os pares $\left(C, \overline{g^{\prime}}\right)$ e uma relação de bordismo análoga à relação anterior, obtemos novamente o grupo de bordismo framed $\Omega_{m-n}^{f r}$. Consideremos o homomorfismo estabilização, stab $: \Omega_{m-n} \rightarrow \Omega_{m-n}^{f r}$, dado por $\left[C, g^{\prime}\right] \mapsto\left[C, \overline{g^{\prime}}\right]$.

Consideremos o homomorfismo $E: \pi_{m}\left(S^{n}\right) \rightarrow \pi_{m+1}\left(S^{n+1}\right)$ induzido pela operação de suspensão. Pelo teorema da suspensão de Freudenthal (vide [Whg-78], página 369), temos que $E$ é isomorfismo para $m<2 n-1$ e é epimorfismo para $m=2 n-1$. Seja $\pi_{k}^{S} \cong \pi_{k+i}\left(S^{i}\right)$, com $k<i-1$, o k-ésimo grupo de homotopia estável das esferas e seja $E^{\infty}: \pi_{m}\left(S^{n}\right) \rightarrow \pi_{m-n}^{S}$ 
uma composição adequada de homomorfismos E. Notemos que $E^{\infty}$ é isomorfismo para $m<2 n-1$ e é epimorfismo para $m=2 n-1$.

Consideremos o homomorfismo $P T_{u}: \pi_{m}\left(S^{n}\right) \rightarrow \Omega_{m-n}$ dado por $[f] \mapsto$ $\left[f^{-1}\left(y_{0}\right), g^{\prime}\right]$, onde supomos $f$ diferenciável, $y_{0}$ um valor regular de $f$ (donde $f^{-1}\left(y_{0}\right) \subset S^{m}$ é uma subvariedade de $S^{m}$ de codimensão $\left.n\right)$ e onde $g^{\prime}$ : $\nu\left(f^{-1}\left(y_{0}\right), S^{m}\right) \rightarrow \mathbb{R}^{n}$ é uma trivialização do fibrado normal $\nu\left(f^{-1}\left(y_{0}\right), S^{m}\right)$. Definindo $P T_{s}: \pi_{m-n}^{S} \rightarrow \Omega_{m-n}^{f r}$ por $[f] \mapsto\left[f^{-1}\left(y_{0}\right), \overline{g^{\prime}}\right]$, temos que PT $:=P T_{s}$ é um isomorfismo, chamado de isomorfismo de Pontryagin-Thom.

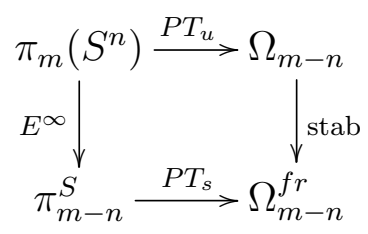

Exemplo 109. Temos que $\Omega_{1}^{f r} \cong \pi_{1}^{S} \cong \mathbb{Z}_{2}$. Sejam $y_{0}$ um ponto e $\Omega_{1}^{f r}=$ $\Omega_{1}\left(y_{0} ; \mathbb{R}\right)$. Então podemos considerar $\mathcal{F}_{1}:=\left(S^{1}, y_{0}, F_{1}\right), \mathcal{F}_{2}:=\left(S^{1}, y_{0}, F_{2}\right)$ tais que $\left[\mathcal{F}_{1}\right],\left[\mathcal{F}_{2}\right] \in \Omega_{1}\left(y_{0} ; \mathbb{R}\right)$, sendo $F_{1}, F_{2}: \tau_{S^{1}} \oplus \nu\left(S^{1}, \mathbb{R}^{2}\right) \rightarrow S^{1} \times \mathbb{R}^{2}$ tais que

$$
F_{1}:\left\{\begin{array}{rl}
\left(x, v_{x}\right) & \mapsto\left(x, v_{x}\right), \\
\left(x, n_{x}\right) & \mapsto\left(x, n_{x}\right),
\end{array} \quad F_{2}:\left\{\begin{aligned}
\left(x, v_{x}\right) & \mapsto\left(x, e_{1}\right), \\
\left(x, n_{x}\right) & \mapsto\left(x, e_{2}\right),
\end{aligned}\right.\right.
$$

onde $v_{x} \in T_{x} S^{1} \cong \mathbb{R}$ e $n_{x} \in\left(T_{x} S^{1}\right)^{\perp} \cong \mathbb{R}$. Seja $G: \tau_{D^{2}} \rightarrow D^{2} \times \mathbb{R}^{2}$ dado por $\left(x, v_{x}\right) \stackrel{G}{\mapsto}\left(x, e_{1}\right),\left(x, n_{x}\right) \stackrel{G}{\mapsto}\left(x, e_{2}\right)$, onde $n_{x} \perp v_{x}$. Notemos que $\left[\mathcal{F}_{2}\right]=0$, pois $\left(D^{2}, y_{0}, G\right)$ é tal que $\partial D^{2}=S^{1}, y_{0 \mid S^{1}}=y_{0}$ e $G_{\mid S^{1}}=F_{2}$ e, por outro lado, $\left[\mathcal{F}_{1}\right] \neq 0$ gera $\Omega_{1}^{\text {fr }}$. 
O próximo resultado nos fornece uma sequência exata, envolvendo grupos de bordismo normal e grupos de homologia com coeficientes torcidos. Este resultado, apresentado aqui de maneira conveniente, pode ser encontrado na íntegra em [K-81] (teorema 9.3).

Teorema 110. Seja X um espaço topológico conexo por caminhos, paracompacto que tem o mesmo tipo de homotopia de um $C W$-complexo de tipo finito. Seja $\varphi=\varphi^{+}-\varphi^{-}$um fibrado virtual sobre $X$. Então a sequência

$$
\cdots \rightarrow \Omega_{2}(X ; \varphi) \stackrel{\mu}{\rightarrow} H_{2}\left(X ; \widetilde{\mathbb{Z}}_{\varphi}\right) \rightarrow \mathbb{Z}_{2} \stackrel{\delta_{1}}{\rightarrow} \Omega_{1}(X ; \varphi) \stackrel{\mu}{\rightarrow} H_{1}\left(X ; \widetilde{\mathbb{Z}}_{\varphi}\right) \rightarrow 0
$$

é exata, onde $\mu\left(\left[M^{m}, g, \bar{g}\right]\right)=g_{*}\left(\left[M^{m}\right]\right)$, com $\left[M^{m}\right] \in H_{m}\left(M ; \widetilde{\mathbb{Z}}_{\tau_{M}}\right)$ sendo a classe fundamental de $M$ e $\widetilde{\mathbb{Z}}_{\tau_{M}}=\widetilde{\mathbb{Z}}_{g^{*}(\varphi)}$. Ainda, $\delta_{1}(1)$ é representado pelo círculo unitário invariantemente paralelizado.

Observação 111. Note que, na sequência apresentada, podemos ver $\mathbb{Z}_{2}$ como sendo $\Omega_{1}^{f r}$ e $\delta_{1}(1)$ como sendo $i_{*}\left(\left[\mathcal{F}_{1}\right]\right)=\left[S^{1}, i \circ y_{0}: S^{1} \rightarrow X, F_{1}\right] \in \Omega_{1}(X ; \varphi)$, onde $i: y_{0} \hookrightarrow X$ é a inclusão e a tripla $\mathcal{F}_{1}$ é dada pelo exemplo 109.

Observação 112. Seja X um espaço topológico conexo por caminhos, paracompacto que tem o mesmo tipo de homotopia de um $C W$-complexo. Notemos que, se $\pi_{n}(X)$ é abeliano finitamente gerado, para todo $n \geqslant 1$, então, pelo lema 49, X tem o mesmo tipo de homotopia de um $C W$-complexo de tipo finito. 


\subsection{Bordismo e coincidências}

Em toda a seção, sejam $X$ uma $m$-variedade fechada, conexa, e $Y$ uma $n$ variedade sem bordo, conexa, com $m, n \geqslant 1$. Sejam $f, f_{1}, f_{2}: X^{m} \rightarrow Y^{n}$ aplicações contínuas.

Nesta seção, inicialmente apresentamos os invariantes homotópicos (como definidos em [K-04] e [K-06]), que relacionam a teoria de bordismo e a teoria de coincidência, que são utilizados no trabalho.

Em seguida, apresentamos alguns resultados tipo Wecken e vemos, para o caso de codimensão 1, em quais situações ainda é necessária uma análise.

Por fim, analisamos os invariantes apresentados no caso especial de raízes e auto-coincidências.

\subsubsection{Sobre o invariante $\omega\left(f_{1}, f_{2}\right)$}

Consideremos uma aproximação, $\left(f_{1}^{\prime}, f_{2}^{\prime}\right): X \rightarrow Y \times Y$, de $\left(f_{1}, f_{2}\right)$, diferenciável e transversa à diagonal $\Delta \subset Y \times Y$. Então $C:=\left(f_{1}^{\prime}, f_{2}^{\prime}\right)^{-1}(\Delta)$ é uma subvariedade de $X$ de dimensão $m-n$ e a inclusão $g: C \hookrightarrow X$ é um mergulho. Consideremos o diagrama geométrico abaixo.

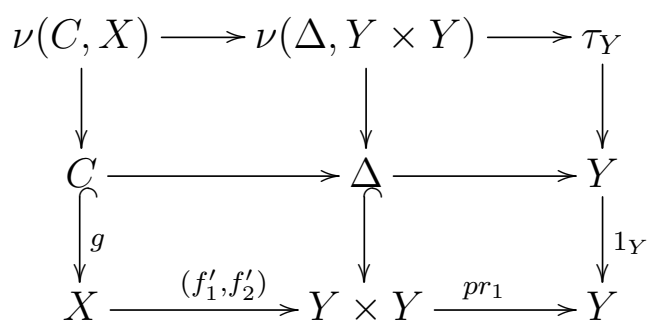

Daí, obtemos o seguinte isomorfismo de fibrados vetoriais (induzido por $\left.\left(f_{1}^{\prime}, f_{2}^{\prime}\right)\right)$ :

$$
\nu(C, X) \cong\left(f_{1}^{\prime}, f_{2}^{\prime}\right)^{*}(\nu(\Delta, Y \times Y)) \cong f_{1}^{\prime *}\left(\tau_{Y}\right)_{\mid C}=g^{*}\left(f_{1}^{\prime *}\left(\tau_{Y}\right)\right)
$$


Para maiores detalhes, vide [MiSta-74] (lema 11.5, página 121). Como $\tau_{C} \oplus$ $\nu(C, X) \cong \tau_{X \mid C}$, segue, usando a identificação acima, que $\tau_{C} \oplus g^{*}\left(f_{1}^{\prime *}\left(\tau_{Y}\right)\right) \cong$ $g^{*}\left(\tau_{X}\right)$. Seja $\bar{g}: \tau_{C} \oplus g^{*}\left(f_{1}^{\prime *}\left(\tau_{Y}\right)\right) \rightarrow g^{*}\left(\tau_{X}\right)$ o isomorfismo estável de fibrados vetoriais sobre $C$ dado pelo mergulho $g$. Assim, $(C, g, \bar{g})$ é uma $\varphi$-variedade fechada singular em $X$ de dimensão $m-n$, onde $\varphi:=f_{1}^{\prime *}\left(\tau_{Y}\right)-\tau_{X}$. Desde que $f_{1} \simeq f_{1}^{\prime}$, podemos considerar $f_{1}^{*}\left(\tau_{Y}\right)$ ao invés de $f_{1}^{\prime *}\left(\tau_{Y}\right)$.

Definição 113. Com as notações acima, definimos $\omega\left(f_{1}, f_{2}\right):=[C, g, \bar{g}] \in$ $\Omega_{m-n}(X ; \varphi)$, onde $\varphi=f_{1}^{*}\left(\tau_{Y}\right)-\tau_{X}$.

Por [K-04], segue que $\omega\left(f_{1}, f_{2}\right)$ é bem-definido e é um invariante homotópico (a menos de isomorfismo). Notemos que, geralmente, o grupo $\Omega_{m-n}(X ; \varphi)$ varia junto com o invariante $\omega\left(f_{1}, f_{2}\right)$, pois $\varphi=f_{1}^{*}\left(\tau_{Y}\right)-\tau_{X}$.

Ainda, também por [K-04], se $\left(f_{1}, f_{2}\right)$ é loose, então $\omega\left(f_{1}, f_{2}\right)=0$.

\subsubsection{Sobre o invariante $\tilde{\omega}\left(f_{1}, f_{2}\right)$}

Seja $E\left(f_{1}, f_{2}\right):=\left\{(x, \theta) \in X \times Y^{I} \mid \theta(0)=f_{1}(x), \theta(1)=f_{2}(x)\right\}$. Temos que $E\left(f_{1}, f_{2}\right)$ é o pullback de $p$ por $\left(f_{1}, f_{2}\right)$, onde $p: Y^{I} \rightarrow Y \times Y$ é a fibração dada por $p(\theta)=(\theta(0), \theta(1))$ (vide [Sp-66], corolário 3, página 98). Consideremos o diagrama geométrico abaixo.

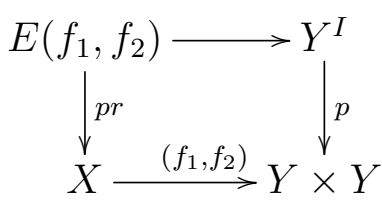

Notemos que se $x_{0} \in X$ é tal que $x_{0} \in \operatorname{Coin}\left(f_{1}, f_{2}\right)$ e $y_{0}:=f_{1}\left(x_{0}\right)=$ $f_{2}\left(x_{0}\right)$, então $p r^{-1}\left(x_{0}\right) \approx p^{-1}\left(f_{1}\left(x_{0}\right), f_{2}\left(x_{0}\right)\right)=\Omega\left(Y, y_{0}\right)$ é a fibra sobre $x_{0}$ de $p r$. Ainda, $E\left(f_{1}, f_{2}\right)$ é um espaço topológico que, em geral, não é conexo por caminhos. De fato, da sequência de homotopia da fibração $p r$, obtemos em particular que o conjunto $\pi_{0}\left(E\left(f_{1}, f_{2}\right)\right)$ está em bijeção com o conjunto das 
classes de Reidemeister $\frac{\pi_{1}(Y)}{\sim}$, isto é, $\# \pi_{0}\left(E\left(f_{1}, f_{2}\right)\right)=R\left(f_{1}, f_{2}\right)$. Ainda, se $F_{i}: f_{i} \simeq f_{i}^{\prime}, i=1,2$, notemos que $E\left(f_{1}, f_{2}\right)$ e $E\left(f_{1}^{\prime}, f_{2}^{\prime}\right)$ têm o mesmo tipo de homotopia sobre $X$, via $(x, \theta) \mapsto\left(x,\left(F_{1}\left(x,_{-}\right)\right)^{-1} * \theta * F_{2}\left(x,{ }_{-}\right)\right)$. Finalmente, observemos que $E\left(f_{1}, f_{2}\right)$ e $E\left(f_{2}, f_{1}\right)$ são homeomorfos sobre $X$, via $(x, \theta) \mapsto$ $\left(x, \theta^{-1}\right)$.

Consideremos novamente uma aproximação, $\left(f_{1}^{\prime}, f_{2}^{\prime}\right)$, de $\left(f_{1}, f_{2}\right)$, diferenciável e transversa à diagonal $\Delta$ e o seguinte diagrama geométrico

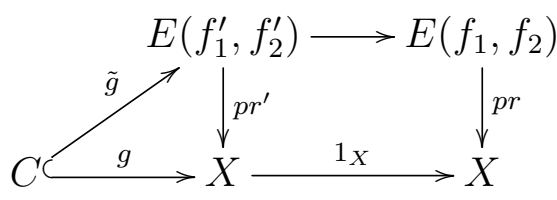

onde $C=\operatorname{Coin}\left(f_{1}^{\prime}, f_{2}^{\prime}\right), \tilde{g}(x)=\left(x, \operatorname{cte}_{f_{1}^{\prime}(x)}\right)$ e $p r^{\prime}(x, \theta)=x$. Notemos que o isomorfismo estável $\bar{g}$ dado por $g$ é tal que $\bar{g}: \tau_{C} \oplus \tilde{g}^{*}\left(p r^{\prime *}\left(f_{1}^{\prime *}\left(\tau_{Y}\right)\right)\right) \rightarrow \tilde{g}^{*}\left(p r^{\prime *}\left(\tau_{X}\right)\right)$. Assim, $(C, \tilde{g}, \bar{g})$ é uma $\tilde{\varphi}$-variedade fechada singular em $E\left(f_{1}^{\prime}, f_{2}^{\prime}\right)$ de dimensão $m-n$, onde $\tilde{\varphi}:=p r^{\prime *}(\varphi)=p r^{\prime *}\left(f_{1}^{\prime *}\left(\tau_{Y}\right)\right)-p r^{\prime *} \tau_{X}$. Desde que $f_{1} \simeq f_{1}^{\prime}$ e $f_{2} \simeq f_{2}^{\prime}$, podemos considerar $f_{1}^{*}\left(\tau_{Y}\right)$ ao invés de $f_{1}^{\prime *}\left(\tau_{Y}\right)$ e $p r: E\left(f_{1}, f_{2}\right) \rightarrow X$ ao invés de $p r^{\prime}: E\left(f_{1}^{\prime}, f_{2}^{\prime}\right) \rightarrow X$.

Definição 114. Com as notações acima, definimos $\tilde{\omega}\left(f_{1}, f_{2}\right):=[C, \tilde{g}, \bar{g}] \in$ $\Omega_{m-n}\left(E\left(f_{1}, f_{2}\right) ; \tilde{\varphi}\right)$, onde $\tilde{\varphi}=p r^{*}\left(f_{1}^{*}\left(\tau_{Y}\right)\right)-p r^{*} \tau_{X}$.

Por [K-06], vemos que $\tilde{\omega}\left(f_{1}, f_{2}\right)$ é bem-definido e é um invariante homotópico (a menos de isomorfismo). Ainda, $\tilde{\omega}\left(f_{1}, f_{2}\right)=0$ se, e só se, $\tilde{\omega}\left(f_{2}, f_{1}\right)=0$. Notemos novamente que, em geral, o grupo $\Omega_{m-n}\left(E\left(f_{1}, f_{2}\right) ; \tilde{\varphi}\right)$ varia junto com o invariante $\tilde{\omega}\left(f_{1}, f_{2}\right)$, pois $\tilde{\varphi}$ e $E\left(f_{1}, f_{2}\right)$ também dependem das classes de homotopia de $f_{1}$ e de $f_{2}$.

Ainda, do mesmo modo que antes, se $\left(f_{1}, f_{2}\right)$ é loose, então $\tilde{\omega}\left(f_{1}, f_{2}\right)=0$. 


\subsubsection{Sobre o número de Nielsen $\tilde{N}\left(f_{1}, f_{2}\right)$}

Sejam $x_{0}, x_{1} \in \operatorname{Coin}\left(f_{1}, f_{2}\right) \subset X$. Por [K-06], notemos que $\tilde{g}\left(x_{0}\right)$ e $\tilde{g}\left(x_{1}\right)$ pertencem à mesma componente conexa por caminhos de $E\left(f_{1}, f_{2}\right)$ se, e somente se, $x_{0}$ e $x_{1}$ são Nielsen equivalentes, isto é, existe $\sigma \in X^{I}$ tal que $\sigma(0)=x_{0}$, $\sigma(1)=x_{1}$ e $f_{1} \circ \sigma \simeq_{\partial I} f_{2} \circ \sigma$. Por abuso de notação, seja $\pi_{0}\left(E\left(f_{1}, f_{2}\right)\right)$ o conjunto das componentes conexas por caminhos de $E\left(f_{1}, f_{2}\right)$. Desde que

$$
\Omega_{m-n}\left(E\left(f_{1}, f_{2}\right) ; \tilde{\varphi}\right) \cong \bigoplus_{A \in \pi_{0}\left(E\left(f_{1}, f_{2}\right)\right)} \Omega_{m-n}\left(A ; \tilde{\varphi}_{\mid A}\right)
$$

podemos considerar as componentes $\tilde{\omega}_{A}\left(f_{1}, f_{2}\right) \in \Omega_{m-n}(A ; \tilde{\varphi} \mid A)$ de $\tilde{\omega}\left(f_{1}, f_{2}\right) \in$ $\Omega_{m-n}\left(E\left(f_{1}, f_{2}\right) ; \tilde{\varphi}\right)$ dadas por $\left[C_{A}, \tilde{g}_{\mid C_{A}}, \bar{g}_{\mid C_{A}}\right]$, onde $C_{A}:=\tilde{g}^{-1}(A)$, para cada $A \in \pi_{0}\left(E\left(f_{1}, f_{2}\right)\right)$.

Definição 115. Seja A uma componente conexa por caminhos de $E\left(f_{1}, f_{2}\right)$. Dizemos que $A$ é essencial se $\tilde{\omega}_{A}\left(f_{1}, f_{2}\right) \neq 0 \in \Omega_{m-n}\left(A ; \tilde{\varphi}_{\mid A}\right)$ e chamamos o número de componentes conexas por caminhos essenciais de $E\left(f_{1}, f_{2}\right)$ de número de Nielsen de $\left(f_{1}, f_{2}\right)$ e o denotamos por $\tilde{N}\left(f_{1}, f_{2}\right)$.

Por $[\mathrm{K}-06]$, temos que $\tilde{N}\left(f_{1}, f_{2}\right)$ é bem-definido.

Teorema 116. Sejam $f_{1}, f_{2}: X^{m} \rightarrow Y^{n}$ aplicações contínuas. Então

- $\tilde{N}\left(f_{1}, f_{2}\right)$ depende apenas das classes de homotopia de $f_{1}$ e de $f_{2}$;

- $\tilde{N}\left(f_{1}, f_{2}\right)=\tilde{N}\left(f_{2}, f_{1}\right)$;

- $\tilde{N}\left(f_{1}, f_{2}\right) \leqslant M C C\left(f_{1}, f_{2}\right)<\infty$;

- $M C C\left(f_{1}, f_{2}\right) \leqslant R\left(f_{1}, f_{2}\right)$ se $n \neq 2$.

Tal resultado pode ser encontrado em [K-06] (teorema 1.9). Em particular, notemos que $\tilde{N}\left(f_{1}, f_{2}\right)$ é um limitante inferior para $M C C\left(f_{1}, f_{2}\right)$. Ainda, observemos que $\tilde{\omega}\left(f_{1}, f_{2}\right)=0$ se, e somente se, $\tilde{N}\left(f_{1}, f_{2}\right)=0$. 
Observação 117. Observe o uso da notação $\tilde{N}\left(f_{1}, f_{2}\right)$ (ver [K-11]) ao invés da notação $N\left(f_{1}, f_{2}\right)$, conforme denotado inicialmente em [K-06].

Uma parte do trabalho é dedicada ao cálculo de $\tilde{N}\left(f_{1}, f_{2}\right)$.

\subsubsection{Resultados tipo Wecken}

Um resultado tipo Wecken é um resultado que, sob certas hipóteses sobre os espaços, conclui que o número mínimo de componentes de coincidências de $\left(f_{1}, f_{2}\right)$ é igual ao número de Nielsen de $\left(f_{1}, f_{2}\right)$, ou seja, $\operatorname{MCC}\left(f_{1}, f_{2}\right)=$ $\tilde{N}\left(f_{1}, f_{2}\right)$, para todo par $\left(f_{1}, f_{2}\right)$ de aplicações contínuas.

Aqui, apresentamos, de maneira geral, dois resultados tipo Wecken e, a seguir, enfatizamos o caso de codimensão 1.

Definição 118. Dizemos que o par $\left(f_{1}, f_{2}\right)$ satisfaz a propriedade de Wecken, se $M C C\left(f_{1}, f_{2}\right)=\tilde{N}\left(f_{1}, f_{2}\right)$.

Notemos, em particular, que, se $\left(f_{1}, f_{2}\right)$ satisfaz a propriedade de Wecken, então $\left(f_{1}, f_{2}\right)$ é loose se, e somente se, $\tilde{N}\left(f_{1}, f_{2}\right)=0$.

A seguir, apresentamos um resultado tipo Wecken, que pode ser encontrado em [K-06] (teorema 1.10).

Teorema 119. Seja $m<2 n-2$. Sejam $f_{1}, f_{2}: X^{m} \rightarrow Y^{n}$ aplicações contínuas. Então $\operatorname{MCC}\left(f_{1}, f_{2}\right)=\tilde{N}\left(f_{1}, f_{2}\right)$.

Observação 120. Se $\left(f_{1}, f_{2}\right)$ é loose, então $\left(f_{1}, f_{2}\right)$ satisfaz a propriedade de Wecken. De fato, inicialmente, lembremos que $\left(f_{1}, f_{2}\right)$ é loose se, e só se, $\operatorname{MCC}\left(f_{1}, f_{2}\right)=0$. Ainda, se $\left(f_{1}, f_{2}\right)$ é loose, então $\tilde{\omega}\left(f_{1}, f_{2}\right)=0$. Finalmente, lembremos que $\tilde{\omega}\left(f_{1}, f_{2}\right)=0$ se, e só se, $\tilde{N}\left(f_{1}, f_{2}\right)=0$. Assim, se $\left(f_{1}, f_{2}\right)$ é loose, então $M C C\left(f_{1}, f_{2}\right)=0=\tilde{N}\left(f_{1}, f_{2}\right)$ e, em particular, $\left(f_{1}, f_{2}\right)$ satisfaz a propriedade de Wecken. 
A seguir, apresentamos mais um resultado tipo Wecken.

Teorema 121. Seja $n=1$. Sejam $f_{1}, f_{2}: X^{m} \rightarrow Y^{1}$ aplicações contínuas. Então $M C C\left(f_{1}, f_{2}\right)=\tilde{N}\left(f_{1}, f_{2}\right)$.

Demonstração. Com efeito, se $n=1$, então ou $Y=\mathbb{R}$, ou $Y=S^{1}$. Se $Y=\mathbb{R}$, então $\left(f_{1}, f_{2}\right)$ é loose, pois existem $y_{1}, y_{2} \in \mathbb{R}, y_{1} \neq y_{2}$, tais que $f_{1} \simeq y_{1}$ e $f_{2} \simeq y_{2}$. Daí, pela observação $120, \operatorname{MCC}\left(f_{1}, f_{2}\right)=0=\tilde{N}\left(f_{1}, f_{2}\right)$. Se $Y=S^{1}$, então o resultado segue por [K-06] (teorema 1.13).

Observação 122. Notemos que, se $Y=S^{1}$, então, por [K-06] (teorema 1.13), temos uma maneira homológica para calcularmos o número de $\mathrm{Ni}$ elsen do par $\left(f_{1}, f_{2}\right)$, a saber, $\tilde{N}\left(f_{1}, f_{2}\right)$ é tal que $\left(f_{1_{*}}-f_{2_{*}}\right)\left(H_{1}(X ; \mathbb{Z})\right)=$ $\tilde{N}\left(f_{1}, f_{2}\right) H_{1}\left(S^{1} ; \mathbb{Z}\right)$.

A partir dos resultados anteriores, obtemos o seguinte resultado tipo Wecken para o caso de codimensão 1:

Corolário 123. Seja $n=1$ ou $n \geqslant 4$. Sejam $f_{1}, f_{2}: X^{n+1} \rightarrow Y^{n}$ aplicações contínuas. Então $\operatorname{MCC}\left(f_{1}, f_{2}\right)=\tilde{N}\left(f_{1}, f_{2}\right)$.

Demonstração. De fato, se $n=1$, então o resultado segue pelo teorema 121. Se $n \geqslant 4$, então $2 n \geqslant n+4$ e portanto $2 n-2>n+1$, donde o resultado segue pelo teorema 119 .

Assim, resta analisar, para o caso de codimensão 1, a existência de resultados tipo Wecken apenas quando $n=2,3$. Em particular, uma parte do trabalho é dedicada a esta análise.

\subsubsection{Raízes e bordismo}

Nesta subseção, estamos interessados em estudar os invariantes definidos anteriormente, para pares de aplicações contínuas da forma $\left(y_{0}, f\right)$, sendo 
$y_{0}$ a aplicação constante igual a $y_{0} \in Y$.

Observemos que, como $y_{0}^{*}\left(\tau_{Y}\right)$ é trivial, então $\omega\left(y_{0}, f\right) \in \Omega_{m-n}\left(X ;-\tau_{X}\right)$ e $\tilde{\omega}\left(y_{0}, f\right) \in \Omega_{m-n}\left(E\left(y_{0}, f\right) ;-p r^{*}\left(\tau_{X}\right)\right)$. Ainda, notemos que $R\left(y_{0}, f\right)=$ $\# \pi_{0}\left(E\left(y_{0}, f\right)\right)=\left[\pi_{1}(Y): f_{\#}\left(\pi_{1}(X)\right)\right]$. Agora, a fim de estudarmos $\tilde{N}\left(y_{0}, f\right)$, vamos analisar as componentes conexas por caminhos de $E\left(y_{0}, f\right)$. Seja $x_{0} \in X$ tal que $f\left(x_{0}\right)=y_{0}$. Consideremos o diagrama geométrico

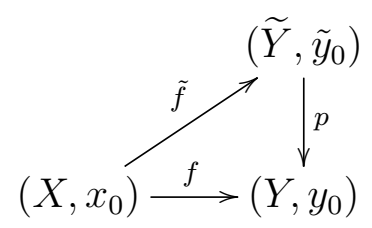

onde $p: \widetilde{Y} \rightarrow Y$ é o revestimento de $Y$ dado por $f_{\#}\left(\pi_{1}(X)\right)=p_{\#}\left(\pi_{1}(\tilde{Y})\right)$ e $\tilde{f}$ é o levantamento de $f$ tal que $\tilde{f}\left(x_{0}\right)=\tilde{y}_{0}$. Desta forma, obtemos a seguinte descrição das componentes conexas por caminhos de $E\left(y_{0}, f\right)$ e a seguinte decomposição do grupo $\Omega_{m-n}\left(E\left(y_{0}, f\right) ; \tilde{\varphi}\right)$ em soma direta:

Lema 124. Com as notações acima, a menos de homeomorfismo, as componentes conexas por caminhos de $E\left(y_{0}, f\right)$ são todas da forma $E(\tilde{y}, \tilde{f})$, com $\tilde{y} \in p^{-1}\left(y_{0}\right)$, e $R\left(y_{0}, f\right)=\# p^{-1}\left(y_{0}\right)$. Ainda, temos que $\Omega_{m-n}\left(E\left(y_{0}, f\right) ; \tilde{\varphi}\right) \cong$ $\bigoplus_{p^{-1}\left(y_{0}\right)} \Omega_{m-n}\left(E\left(\tilde{y}_{0}, \tilde{f}\right) ; \tilde{\varphi}_{\mid E\left(\tilde{y}_{0}, \tilde{f}\right)}\right)$.

Demonstração. De fato, por [K-062] (lema 4.1), segue que $E(\tilde{y}, \tilde{f})$ é conexo por caminhos, para todo $\tilde{y} \in p^{-1}\left(y_{0}\right)$. Ainda, desde que dado $(x, \theta) \in$ $E\left(y_{0}, f\right)$, existe um único caminho $\tilde{\theta}: I \rightarrow \widetilde{Y}$ que termina em $\tilde{f}(x)$ e é tal que $p \circ \tilde{\theta}=\theta$ (vide [Ma-77], página 151), então $E\left(y_{0}, f\right) \ni(x, \theta) \mapsto(x, \tilde{\theta}) \in$ $\dot{\cup}_{\tilde{y} \in p^{-1}\left(y_{0}\right)} E(\tilde{y}, \tilde{f})$ define um homeomorfismo. Em particular, a menos de homeomorfismo, $E(\tilde{y}, \tilde{f})$ são as componentes conexas por caminhos de $E\left(y_{0}, f\right)$, e $R\left(y_{0}, f\right)=\# \pi_{0}\left(E\left(y_{0}, f\right)\right)=\# p^{-1}\left(y_{0}\right)$ (vide [K-062], corolário 4.2). Notemos ainda que, dados $\tilde{y}_{1}, \tilde{y}_{2} \in p^{-1}\left(y_{0}\right), E\left(\tilde{y}_{1}, \tilde{f}\right)$ e $E\left(\tilde{y}_{2}, \tilde{f}\right)$ têm o mesmo tipo de homotopia (sobre $X$ ), via $(x, \tilde{\theta}) \mapsto(x, \tilde{\tau} * \tilde{\theta})$, onde $\tilde{\tau}$ é um caminho que liga 
$\tilde{y}_{2}$ a $\tilde{y}_{1}$. Desta forma, $\Omega_{m-n}\left(E\left(y_{0}, f\right) ; \tilde{\varphi}\right) \cong \bigoplus_{p^{-1}\left(y_{0}\right)} \Omega_{m-n}\left(E\left(\tilde{y}_{0}, \tilde{f}\right) ; \tilde{\varphi}_{\mid E\left(\tilde{y}_{0}, \tilde{f}\right)}\right)$.

Ainda, temos a seguinte descrição das componentes de $\tilde{\omega}\left(y_{0}, f\right)$ :

Lema 125. A menos de isomorfismo, por abuso de notação, as componentes de $\tilde{\omega}\left(y_{0}, f\right)$ são todas iguais a $\tilde{\omega}\left(\tilde{y}_{0}, \tilde{f}\right)$. Ainda, se $p^{-1}\left(y_{0}\right)$ é infinito, então elas são todas iguais $a=\tilde{\omega}\left(\tilde{y}_{0}, \tilde{f}\right)$.

Demonstração. Com efeito, por [K-062] (demonstração do teorema 4.3), segue, a menos de isomorfismo, que as componentes $\tilde{\omega}_{E(\tilde{y}, \tilde{f})}\left(y_{0}, f\right)$ de $\tilde{\omega}\left(y_{0}, f\right)$ são todas iguais a $\tilde{\omega}\left(\tilde{y}_{0}, \tilde{f}\right) \in \Omega_{m-n}\left(E\left(\tilde{y}_{0}, \tilde{f}\right) ; \tilde{\varphi}_{\mid E\left(\tilde{y}_{0}, \tilde{f}\right)}\right)$. Por [K-062] (proposição 4.6), se $\# p^{-1}\left(y_{0}\right)=\infty$, isto é, se $\left[\pi_{1}(Y): f_{\#}\left(\pi_{1}(X)\right)\right]=\left[\pi_{1}(Y)\right.$ : $\left.p_{\#}\left(\pi_{1}(\tilde{Y})\right)\right]=\infty$, então $\left(y_{0}, f\right)$ e $\left(\tilde{y}_{0}, \tilde{f}\right)$ são deformáveis a livre de coincidência e portanto as componentes de ambos são todas nulas.

Observação 126. Em particular, a menos de isomorfismo, por abuso de notação, temos que $\tilde{\omega}\left(y_{0}, f\right) \in \Delta \subset \bigoplus_{p^{-1}\left(y_{0}\right)} \Omega_{m-n}\left(E\left(\tilde{y}_{0}, \tilde{f}\right) ; \tilde{\varphi}_{\mid E\left(\tilde{y}_{0}, \tilde{f}\right)}\right)$. Ainda, $\tilde{N}\left(y_{0}, f\right)=0$ se, e só se, $\tilde{N}\left(\tilde{y}_{0}, \tilde{f}\right)=0$.

O próximo resultado, apresentado aqui de maneira conveniente, pode ser encontrado na íntegra em [K-062] (proposição 1.5).

Proposição 127. Com as notações anteriores, $\tilde{N}\left(y_{0}, f\right)=d . \tilde{N}\left(\tilde{y}_{0}, \tilde{f}\right)$, onde $\left.\left.d=\# p^{-1}\left(y_{0}\right) \in\right] 0, \infty\right]$ e assumimos $\infty .0:=0$.

\subsubsection{Auto-coincidências e bordismo}

Nesta subseção, estamos interessados em estudar os invariantes definidos anteriormente, para pares de aplicações contínuas da forma $(f, f)$.

Notemos que $\omega(f, f) \in \Omega_{m-n}(X ; \varphi)$ e $\tilde{\omega}(f, f) \in \Omega_{m-n}(E(f, f) ; \tilde{\varphi})$. Ainda, $M C C(f, f) \leqslant 1$, pois $C o i n(f, f)=X$ é conexo por caminhos. 


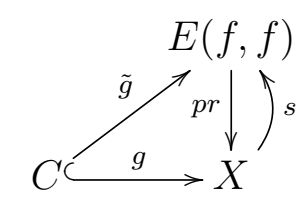

Definindo $s: X \rightarrow E(f, f)$ por $s(x)=\left(x\right.$, cte $\left._{f(x)}\right)$, para todo $x \in X$, obtemos que $s$ é uma seção de $p r$, isto é, $p r \circ s=1_{X}$. Ainda, $s \circ g=\tilde{g}$ e, desde que $X$ é conexo por caminhos, então $\tilde{N}(f, f) \leqslant 1$. Finalmente, observemos que $s_{*}(\omega(f, f))=s_{*}([C, g, \bar{g}])=[C, s \circ g, \bar{g}]=\tilde{\omega}(f, f)$ e, em particular, $\omega(f, f)=0$ se, e só se, $\tilde{\omega}(f, f)=0$.

O próximo resultado nos fornece uma condição suficiente para o par $(f, f)$ ser loose, isto é, deformável a livre de coincidência (ou para $f$ ser deformável a livre de auto-coincidência). Este resultado, apresentado aqui de maneira conveniente, pode ser encontrado na íntegra em [K-04].

Teorema 128. Seja $f: X \rightarrow Y$ uma aplicação contínua. Se $f^{*}\left(\tau_{Y}\right)$ admite uma seção que não se anula, então $(f, f)$ é loose. 


\section{Capítulo 2}

\section{Coincidências em codimensão}

\section{1, sendo o domínio uma esfera}

Em todo o capítulo, sejam $f_{1}, f_{2}: S^{n+1} \rightarrow Y^{n}$ aplicações contínuas, onde $Y$ é uma $n$-variedade sem bordo, conexa, com $n \geqslant 1$ e seja $y_{0} \in Y$.

Neste capítulo, estamos interessados em estudar o invariante $\tilde{\omega}\left(f_{1}, f_{2}\right)$ quando o domínio das aplicações é uma esfera $(n+1)$-dimensional e o contradomínio é uma variedade sem bordo, conexa, $n$-dimensional, lembrando que tal invariante pertence ao grupo de bordismo normal $\Omega_{1}\left(E\left(f_{1}, f_{2}\right) ; \tilde{\varphi}\right)$. Sendo o domínio das aplicações uma esfera de dimensão pelo menos 3, veremos, usando um resultado de Koschorke ([K-06]) que remete a um outro de Hatcher e Quinn ([HaQ-74]), que tal grupo é isomorfo a $\Omega_{1}^{f r}\left(\Omega\left(Y, y_{0}\right)\right)$, deixando então de depender do par $\left(f_{1}, f_{2}\right)$ de aplicações contínuas e do fibrado virtual $\tilde{\varphi}$. Desta forma, os invariantes das aplicações contínuas de $S^{n+1}$ em $Y$ estão todos num mesmo grupo. Além disso, neste contexto, veremos que é possível decompor $\tilde{\omega}\left(f_{1}, f_{2}\right)$ na soma de $\tilde{\omega}\left(f_{1}, f_{1}\right)$, no caso especial de auto-coincidências, com $\tilde{\omega}\left(y_{0}, f_{2}-f_{1}\right)$, no caso especial de raízes. Em seguida, veremos que, para $n$ igual a 2 ou $n$ ímpar, o invariante para 
auto-coincidências se anula, igualando o invariante geral $\tilde{\omega}\left(f_{1}, f_{2}\right)$ com o invariante para raízes $\tilde{\omega}\left(y_{0}, f_{2}-f_{1}\right)$. Desta forma, daremos mais ênfase ao estudo deste último ao invés do invariante geral. Em particular, definiremos dois homomorfismos $\omega^{\prime}: \pi_{n+1}(Y) \rightarrow \mathbb{Z}_{2}$ e $\omega^{\prime \prime}: \pi_{n+1}(Y) \rightarrow \pi_{2}(Y)$ que ajudam no cálculo do número de Nielsen $\tilde{N}\left(y_{0}, f\right)$, para toda aplicação contínua $f: S^{n+1} \rightarrow Y$. Ainda, usando um resultado de Koschorke ([K-81], teorema $9.3)$, nós obtemos (proposição 136) que o grupo $\Omega_{1}^{f r}\left(\Omega\left(Y, y_{0}\right)\right.$ ), sob determinadas hipóteses, pode ser decomposto em soma direta e que, a partir de tal decomposição e dos cálculos de $\omega^{\prime}$ e de $\omega^{\prime \prime}$, é possível recuperar $\tilde{\omega}\left(y_{0}, f\right)$, para toda aplicação contínua $f: S^{n+1} \rightarrow Y$. Daí, estudando tais homomorfismos para superfícies e 3-variedades, nós obtemos um resultado tipo Wecken para $n=2,3$ (teorema 159), o qual diz que o número mínimo de componentes de coincidências $\operatorname{MCC}\left(f_{1}, f_{2}\right)$ é igual ao número de Nielsen $\tilde{N}\left(f_{1}, f_{2}\right)$, para quaisquer aplicações contínuas $f_{1}, f_{2}: S^{n+1} \rightarrow Y$, com $n=2,3$. Daí, juntando com resultados tipo Wecken de Koschorke ([K-06]), enunciamos tal resultado para toda dimensão $n \geqslant 1$. Por fim, nós calculamos $\omega^{\prime \prime}$ para vários espaços $Y$ (proposições 151, 152, 153, 164, 165, 178, entre outros resultados) e, em particular, nós obtemos que tal homomorfismo é identicamente nulo para todo fibrado esférico sobre esfera, com exceção de $S^{2}$-fibrados não triviais sobre $S^{4}$, quando tal homomorfismo, restrito a $\mathbb{Z}$, é injetor (proposição 166 e teorema 171).

\subsection{Particularidades sobre o invariante}

Consideremos o seguinte diagrama geométrico 


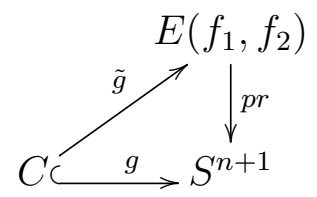

onde $C:=\operatorname{Coin}\left(f_{1}, f_{2}\right), E\left(f_{1}, f_{2}\right)=\left\{(x, \theta) \in S^{n+1} \times Y^{I} \mid \theta(0)=f_{1}(x), \theta(1)=\right.$ $\left.f_{2}(x)\right\}, \tilde{g}(x)=\left(x\right.$, cte $\left._{f_{1}(x)}\right)$ e $\operatorname{pr}(x, \theta)=x$. Temos que o invariante $\tilde{\omega}$ é tal que

$$
\tilde{\omega}\left(f_{1}, f_{2}\right):=[C, \tilde{g}, \bar{g}] \in \Omega_{1}\left(E\left(f_{1}, f_{2}\right) ; \tilde{\varphi}\right)=\bigoplus_{A \in \pi_{0}\left(E\left(f_{1}, f_{2}\right)\right)} \Omega_{1}\left(A ; \tilde{\varphi}_{\mid A}\right),
$$

onde $\tilde{\varphi}:=p r^{*}\left(f_{1}^{*}\left(\tau_{Y}\right)-\tau_{S^{n+1}}\right)$.

Nesta seção, apresentamos vários resultados aplicados para coincidências em codimensão 1, sendo o domínio uma esfera, os quais podem ser encontrados na íntegra em [K-06], [K-062], [K-07], entre outros. Vamos ver que, na verdade, a menos de isomorfismo, o invariante $\tilde{\omega}\left(f_{1}, f_{2}\right)$ pertence a $\Omega_{1}^{f r}\left(\Omega\left(Y, y_{0}\right)\right)$, para quaisquer aplicações contínuas $f_{1}, f_{2}: S^{n+1} \rightarrow Y$, com $n \geqslant 2$. Além disto, vamos ver que é possível operar tais invariantes dentro do grupo e até mesmo decompor tal grupo, sob determinadas hipóteses, em soma direta. Vamos ainda definir e estudar homomorfismos que juntos, em geral, determinam $\tilde{\omega}\left(y_{0}, f\right)$, para toda aplicação contínua $f: S^{n+1} \rightarrow Y$. Finalmente, veremos alguns resultados gerais que implicam na deformabilidade a livre de coincidência de pares de aplicações contínuas.

Observação 129. Desde que $S^{n+1}$ én-conexa, então, por [K-06] (proposição 2.4), existe um isomorfismo $i: \Omega_{k}^{f r}\left(\Omega\left(Y, y_{0}\right)\right) \rightarrow \Omega_{k}\left(E\left(f_{1}, f_{2}\right) ; \tilde{\varphi}\right)$, para todo $k<n$. Temos que $i$ é tal que $i^{-1}:[M, \tilde{g}, \bar{g}] \mapsto\left[M, \tilde{g}^{\prime}, \bar{g}\right]$, onde $\tilde{g}^{\prime}(x)=\left(f_{1} \circ\right.$ $\left.G\left(x,{ }_{-}\right)\right)^{-1} * \theta_{\tilde{g}(x)} *\left(f_{2} \circ G\left(x,{ }_{-}\right)\right), G: M \times I \rightarrow S^{n+1}$ é tal que $G: p r \circ \tilde{g} \simeq c t e_{x_{0}}$, e $\theta_{\tilde{g}(x)}$ é tal que $\tilde{g}(x)=\left(p r \circ \tilde{g}(x), \theta_{\tilde{g}(x)}\right)$.

A partir desta observação, enunciamos o resultado abaixo.

Lema 130. Se $n \geqslant 2$, então existe um isomorfismo $i: \Omega_{1}^{f r}\left(\Omega\left(Y, y_{0}\right)\right) \rightarrow$ $\Omega_{1}\left(E\left(f_{1}, f_{2}\right) ; \tilde{\varphi}\right)$. 
Observação 131. Usando o isomorfismo acima, denotamos $i^{-1}\left(\tilde{\omega}\left(f_{1}, f_{2}\right)\right) \in$ $\Omega_{1}^{f r}\left(\Omega\left(Y, y_{0}\right)\right)$ simplesmente por $\tilde{\omega}\left(f_{1}, f_{2}\right)$. Desta forma, o grupo onde o invariante se encontra não depende mais do par de aplicações onde ele é aplicado, isto é, $\tilde{\omega}\left(f_{1}, f_{2}\right) \in \Omega_{1}^{f r}\left(\Omega\left(Y, y_{0}\right)\right)$, para quaisquer aplicações contínuas $f_{1}, f_{2}: S^{n+1} \rightarrow Y^{n}$, com $n \geqslant 2$.

De agora em diante, nesta seção, seja $n \geqslant 2$. Os três resultados seguintes nos permitem fazer cálculos com o invariante, usando a operação do grupo em que ele se encontra.

Lema 132. $\tilde{\omega}\left(f_{1}, f_{2}\right)=\tilde{\omega}\left(f_{1}, y_{0}\right)+\tilde{\omega}\left(y_{0}, f_{2}\right)$.

Demonstração. Com efeito, temos que existe $f_{i}^{\prime} \simeq f_{i}$ tal que $f_{1}^{\prime}\left(S_{-}^{n+1}\right) \equiv y_{0}$ e $f_{2}^{\prime}\left(S_{+}^{n+1}\right) \equiv y_{0}$. Sem perda de generalidade, suponha que $f_{1}^{\prime}\left(S_{-}^{n+1}\right) \equiv y_{1}$ e $f_{2}^{\prime}\left(S_{+}^{n+1}\right) \equiv y_{2}, \operatorname{com} y_{1} \neq y_{2}$. Assim, $\operatorname{Coin}\left(f_{1}^{\prime}, f_{2}^{\prime}\right)=\operatorname{Coin}\left(f_{1}^{\prime}, y_{2}\right) \dot{\cup}$ $\operatorname{Coin}\left(y_{1}, f_{2}^{\prime}\right)=f_{1}^{\prime-1}\left(y_{2}\right) \dot{\cup} f_{2}^{\prime-1}\left(y_{1}\right)$. Em particular, Coin $\left(f_{1}, f_{2}\right)$ e $f_{1}^{-1}\left(y_{0}\right) \dot{\cup}$ $f_{2}^{-1}\left(y_{0}\right)$ são cobordantes. Da mesma maneira, obtemos as demais identificações e o resultado segue.

Este resultado nos diz que basta conhecermos o invariante ora quando uma das aplicações é constante, ora quando a outra é constante, para conhecermos o invariante para o par de aplicações.

Lema 133. Sejam $f_{1}, f_{1}^{\prime}, f_{2}, f_{2}^{\prime}: S^{n+1} \rightarrow Y$ aplicações contínuas. Então $\tilde{\omega}\left(f_{1}+f_{1}^{\prime}, f_{2}+f_{2}^{\prime}\right)=\tilde{\omega}\left(f_{1}, f_{2}\right)+\tilde{\omega}\left(f_{1}^{\prime}, f_{2}^{\prime}\right)$.

Demonstração. De fato, suponha, sem perda de generalidade, que $\left[f_{i}\right]$, $\left[f_{i}^{\prime}\right] \in \pi_{n+1}\left(Y, y_{i}\right)$, com $y_{1} \neq y_{2}$. Pela definição da operação de grupo em $\pi_{n+1}\left(Y, y_{i}\right)$, temos que $\operatorname{Coin}\left(f_{1}+f_{1}^{\prime}, f_{2}+f_{2}^{\prime}\right)=\operatorname{Coin}\left(f_{1}, f_{2}\right) \cup \operatorname{Coin}\left(f_{1}^{\prime}, f_{2}^{\prime}\right)$. Denote $\tilde{\omega}\left(f_{1}+f_{1}^{\prime}, f_{2}+f_{2}^{\prime}\right):=[C, \tilde{g}, \bar{g}], \tilde{\omega}\left(f_{1}, f_{2}\right):=[D, \tilde{h}, \bar{h}]$ e $\tilde{\omega}\left(f_{1}^{\prime}, f_{2}^{\prime}\right):=$ 
$\left[D^{\prime}, \tilde{h^{\prime}}, \overline{h^{\prime}}\right]$. Pela igualdade anterior, segue que $C$ e $D \cup D^{\prime}$ são cobordantes. Da mesma forma, obtemos $\tilde{g} \sim \tilde{h} \dot{\cup} \tilde{h}^{\prime}, \bar{g} \sim \bar{h} \dot{\cup} \overline{h^{\prime}}$ e o resultado segue.

Este resultado generaliza o anterior na medida em que podemos considerar $f_{2}$ e $f_{1}^{\prime}$ como sendo constantes. O próximo resultado nos ajuda a calcular o invariante quando já conhecemos o cálculo para auto-coincidências e para raízes.

Lema 134. $\tilde{\omega}\left(f_{1}, f_{2}\right)=\tilde{\omega}\left(f_{1}, f_{1}\right)+\tilde{\omega}\left(y_{0}, f_{2}-f_{1}\right)$.

Demonstração. O resultado segue do lema anterior.

Observação 135. Desta forma, podemos considerar o homomorfismo $\tilde{\omega}$ : $\pi_{n+1}(Y) \times \pi_{n+1}(Y) \rightarrow \Omega_{1}^{f r}\left(\Omega\left(Y, y_{0}\right)\right)$ dado por $\tilde{\omega}:\left(\left[f_{1}\right],\left[f_{2}\right]\right) \mapsto \tilde{\omega}\left(f_{1}, f_{2}\right)$.

O resultado seguinte nos permite fazer cálculos com o invariante, através de uma decomposição do grupo onde ele se encontra em soma direta.

Proposição 136. Se cada componente conexa por caminhos de $\Omega\left(Y, y_{0}\right)$ tem o mesmo tipo de homotopia de um $C W$-complexo de tipo finito, então $\left.\Omega_{1}^{f r}\left(\Omega\left(Y, y_{0}\right)\right) \cong\left(\underset{\pi_{1}(Y)}{\bigoplus} \mathbb{Z}_{2}\right) \oplus \underset{\pi_{1}(Y)}{\bigoplus} \pi_{2}(Y)\right)$.

Demonstração. De fato, seja $X$ o conjunto das componentes conexas por caminhos de $\Omega\left(Y, y_{0}\right)$. Então, para cada $A \in X$, pelo teorema 110 e pela observação 111, sendo $\varphi$ trivial, segue que a sequência

$$
\cdots H_{2}(A ; \mathbb{Z}) \rightarrow \Omega_{1}^{f r} \stackrel{i_{*}}{\rightarrow} \Omega_{1}^{f r}(A) \stackrel{\mu}{\rightarrow} H_{1}(A ; \mathbb{Z}) \rightarrow 0
$$

é exata, onde $\Omega_{1}^{f r}=\Omega_{1}^{f r}\left(y_{A}\right)$ e $i: y_{A} \hookrightarrow A$ é a inclusão. Considere $r: A \rightarrow y_{A}$ a aplicação constante. Desde que $r_{*} \circ i_{*}=1_{\Omega_{1}^{f r}}$, então a sequência cinde e portanto $\Omega_{1}^{f r}(A) \cong \Omega_{1}^{f r} \oplus H_{1}(A ; \mathbb{Z}) \cong \mathbb{Z}_{2} \oplus H_{1}(A ; \mathbb{Z})$, para cada $A \in X$. Agora, como as componentes conexas por caminhos de $\Omega\left(Y, y_{0}\right)$ têm o mesmo tipo de homotopia, então, para cada $A \in X, \pi_{1}(A) \cong \pi_{1}\left(\Omega\left(Y, y_{0}\right)\right) \cong \pi_{2}(Y)$. 
Daí, como $\pi_{2}(Y)$ é abeliano, segue, usando a aplicação de Hurewicz, que $H_{1}(A ; \mathbb{Z}) \cong \pi_{1}(A) \cong \pi_{2}(Y)$, para cada $A \in X$. Logo, $\Omega_{1}^{f r}\left(\Omega\left(Y, y_{0}\right)\right) \cong$ $\bigoplus_{A \in X} \Omega_{1}^{f r}(A) \cong\left(\bigoplus_{X} \mathbb{Z}_{2}\right) \oplus\left(\bigoplus_{X} \pi_{2}(Y)\right)=\left(\bigoplus_{\pi_{1}(Y)} \mathbb{Z}_{2}\right) \oplus\left(\bigoplus_{\pi_{1}(Y)} \pi_{2}(Y)\right)$, uma vez que $\# X=\# \pi_{0}\left(\Omega\left(Y, y_{0}\right)\right)=\# \pi_{1}(Y)$.

Observação 137. Sejam X o conjunto das componentes conexas por caminhos de $\Omega\left(Y, y_{0}\right)$ e seja $A \in X$. Consideremos os homomorfismos $r_{*}$ : $\Omega_{1}^{f r}(A) \rightarrow \Omega_{1}^{f r}, \mu: \Omega_{1}^{f r}(A) \rightarrow H_{1}(A ; \mathbb{Z})$ e as identificações $\Omega_{1}^{f r} \cong \mathbb{Z}_{2}$, $H_{1}(A ; \mathbb{Z}) \cong \pi_{2}(Y)$, como na demonstração da proposição acima. Desde que $\Omega_{1}^{f r}\left(\Omega\left(Y, y_{0}\right)\right) \cong \bigoplus_{A \in X} \Omega_{1}^{f r}(A)$, podemos considerar a projeção $p_{A}$ : $\Omega_{1}^{f r}\left(\Omega\left(Y, y_{0}\right)\right) \rightarrow \Omega_{1}^{f r}(A)$, a qual é dada por $\left[C, \tilde{g}^{\prime}, \bar{g}\right] \mapsto\left[C_{A}, \tilde{g}_{\mid C_{A}}^{\prime}, \bar{g}_{\mid C_{A}}\right]$, onde $C_{A}:=\tilde{g}^{\prime-1}(A)$. Notemos que a componente $\tilde{\omega}_{A}\left(f_{1}, f_{2}\right) \in \Omega_{1}^{f r}(A)$ de $\tilde{\omega}\left(f_{1}, f_{2}\right) \in \Omega_{1}^{f r}\left(\Omega\left(Y ; y_{0}\right)\right)$ é tal que $p_{A}\left(\tilde{\omega}\left(f_{1}, f_{2}\right)\right)=\tilde{\omega}_{A}\left(f_{1}, f_{2}\right)$. Desta forma, podemos considerar os homomorfimos $\omega_{A}^{\prime}:=r_{*} \circ p_{A} \circ \tilde{\omega}: \pi_{n+1}(Y) \times \pi_{n+1}(Y) \rightarrow$ $\mathbb{Z}_{2}$ e $\omega_{A}^{\prime \prime}:=\mu \circ p_{A} \circ \tilde{\omega}: \pi_{n+1}(Y) \times \pi_{n+1}(Y) \rightarrow \pi_{2}(Y)$. Daí, observemos que, se cada componente conexa por caminhos de $\Omega\left(Y, y_{0}\right)$ tem o mesmo tipo de homotopia de um CW-complexo de tipo finito, então, pela proposição 110, basta calcularmos $\omega_{A}^{\prime}$ e $\omega_{A}^{\prime \prime}$, para todo $A \in X$, a fim de determinar $\tilde{\omega}\left(f_{1}, f_{2}\right)$.

Observação 138. Se Y tem o mesmo tipo de homotopia de um $C W$-complexo, notemos que $\Omega\left(Y, y_{0}\right)$ também o tem (vide [FrPi-90], página 236). Daí, se $\pi_{k+1}(Y) \cong \pi_{k}\left(\Omega\left(Y, y_{0}\right)\right)$ é finitamente gerado, para todo $k \geqslant 1$, então, pelo lema 49 , obtemos que cada componente conexa por caminhos de $\Omega\left(Y, y_{0}\right)$ tem o mesmo tipo de homotopia de um $C W$-complexo de tipo finito.

Os próximos resultados nos dão condições suficientes para um par de aplicações contínuas ser loose, isto é, deformável a livre de coincidência. Lembremos que, se $\left(f_{1}, f_{2}\right)$ é loose, então $\tilde{\omega}\left(f_{1}, f_{2}\right)=0$.

Lema 139. Se $Y$ é aberta, então $\left(f_{1}, f_{2}\right)$ é loose. 
Demonstração. Com efeito, como $S^{n+1}$ é compacta, então $f_{1}\left(S^{n+1}\right)$, $f_{2}\left(S^{n+1}\right)$ também o são. Daí, como $Y$ é aberta, então existem $y_{1} \in Y \backslash$ $f_{1}\left(S^{n+1}\right)$ e $y_{2} \in Y \backslash f_{2}\left(S^{n+1}\right)$. Em particular, segue que $f_{i}\left(S^{n+1}\right) \subset Y \backslash\left\{y_{i}\right\}$, $i=1,2$. Suponha, sem perda de generalidade, $y_{1} \neq y_{2}$. Considere $f_{1}^{\prime} \simeq f_{1}$ tal que $f_{1}^{\prime}\left(S_{-}^{n+1}\right) \subset Y \backslash y_{1}, f_{1}^{\prime}\left(S_{+}^{n+1}\right) \equiv y_{2}$. Considere ainda $f_{2}^{\prime} \simeq f_{2}$ tal que $f_{2}^{\prime}\left(S_{+}^{n+1}\right) \subset Y \backslash y_{2}, f_{2}^{\prime}\left(S_{-}^{n+1}\right) \equiv y_{1}$. Assim, $\operatorname{Coin}\left(f_{1}^{\prime}, f_{2}^{\prime}\right)=\emptyset$ e portanto $\left(f_{1}, f_{2}\right)$ é loose.

Lema 140. Se $\pi_{1}(Y)$ é infinito, então $\left(f_{1}, f_{2}\right)$ é loose.

Demonstração. De fato, se $\pi_{1}(Y)$ é infinito, então $p^{-1}(y)$ é infinito, para todo $y \in Y$, onde $p: \widetilde{Y} \rightarrow Y$ é o revestimento universal de $Y$. Considere um levantamento $\tilde{f}_{i}: S^{n+1} \rightarrow \tilde{Y}$ de $f_{i}, i=1,2$. Como $S^{n+1}$ é compacta, então $\tilde{f}_{i}\left(S^{n+1}\right)$ também o é e portanto $\tilde{f}_{i}\left(S^{n+1}\right) \cap p^{-1}(y)$ é finito, para todo $y \in Y, i=1,2$. Considere $y_{1}, y_{2} \in Y, y_{1} \neq y_{2}$, e denote $\tilde{f}_{1}\left(S^{n+1}\right) \cap p^{-1}\left(y_{1}\right):=\left\{y_{11}, \ldots, y_{1 k_{1}}\right\}, \tilde{f}_{2}\left(S^{n+1}\right) \cap p^{-1}\left(y_{2}\right):=\left\{y_{21}, \ldots, y_{2 k_{2}}\right\}$. Considere um subconjunto $\left\{z_{i 1}, \ldots, z_{i k_{i}}\right\} \subset \tilde{Y} \backslash \tilde{f}_{i}\left(S^{n+1}\right), i=1,2$. Seja $h$ um difeomorfismo isotópico a $1_{\widetilde{Y}}$ tal que $h\left(z_{i j}\right)=y_{i j}, j=1, \ldots, k_{i}, i=1,2$. Assim, $f_{i} \simeq p \circ\left(h \circ \tilde{f}_{i}\right)=: f_{i}^{\prime}$, com $f_{i}^{\prime}\left(S^{n+1}\right) \subset Y \backslash\left\{y_{i}\right\}$. Agora, sem perda de generalidade, podemos supor que $f_{1}^{\prime}\left(S_{-}^{n+1}\right) \subset Y \backslash y_{1}, f_{1}^{\prime}\left(S_{+}^{n+1}\right) \equiv y_{2}$, $f_{2}^{\prime}\left(S_{+}^{n+1}\right) \subset Y \backslash y_{2}$ e $f_{2}^{\prime}\left(S_{-}^{n+1}\right) \equiv y_{1}$, donde $\operatorname{Coin}\left(f_{1}^{\prime}, f_{2}^{\prime}\right)=\emptyset$ e portanto $\left(f_{1}, f_{2}\right)$ é loose.

Lema 141. Seja $(Y, p, B)$ uma fibração. Se p admite uma seção $s: B \rightarrow Y$, então $\left(f_{1}, f_{2}\right)$ é loose.

Demonstração. Com efeito, seja $F:=p^{-1}\left(b_{0}\right)$, para algum $b_{0} \in B$. Se $p$ admite uma seção $s$, então a sequência abaixo é exata curta e cinde.

$$
0 \rightarrow \pi_{n+1}(F) \stackrel{i_{\#}}{\rightarrow} \pi_{n+1}(Y) \underset{s_{\#}}{\stackrel{p_{\#}}{\rightleftarrows}} \pi_{n+1}(B) \rightarrow 0
$$


Assim, $\pi_{n+1}(Y) \cong \pi_{n+1}(B) \oplus \pi_{n+1}(F)$, pois $n+1 \geqslant 2$. Em particular, se $[f] \in \pi_{n+1}(Y)=s_{\#}\left(\pi_{n+1}(B)\right) \cdot i_{\#}\left(\pi_{n+1}(F)\right)$, então $\operatorname{Im} f^{\prime} \subset \operatorname{Im} s \cup \operatorname{Im} i \subsetneq Y$, para alguma $f^{\prime} \simeq f$. Daí, sem perda de generalidade, sejam $y_{1}, y_{2} \in Y, y_{1} \neq$ $y_{2}$, tais que $f_{i}^{\prime}\left(S^{n+1}\right) \subset Y \backslash y_{i}$, para alguma $f_{i}^{\prime} \simeq f_{i}, i=1,2$. Suponhamos ainda que $f_{1}^{\prime}\left(S_{-}^{n+1}\right) \subset Y \backslash y_{1}, f_{1}^{\prime}\left(S_{+}^{n+1}\right) \equiv y_{2}, f_{2}^{\prime}\left(S_{+}^{n+1}\right) \subset Y \backslash y_{2}, f_{2}^{\prime}\left(S_{-}^{n+1}\right) \equiv y_{1}$. $\operatorname{Logo}, \operatorname{Coin}\left(f_{1}^{\prime}, f_{2}^{\prime}\right)=\emptyset$ e portanto $\left(f_{1}, f_{2}\right)$ é loose.

Observação 142. Em particular, se $Y=Y_{1} \times Y_{2}$, para algum $Y_{1}, Y_{2}$, então $\left(f_{1}, f_{2}\right)$ é loose.

O próximo resultado nos diz que, sob determinadas hipóteses, o cálculo do invariante para um par arbitrário de aplicações contínuas se reduz ao cálculo do invariante para um par no caso especial de raízes.

Lema 143. Se $n=2$, ou $n$ é ímpar, então $\tilde{\omega}\left(f_{1}, f_{2}\right)=\tilde{\omega}\left(y_{0}, f_{2}-f_{1}\right)$.

Demonstração. De fato, se $Y$ é aberta, então, pelo lema $139,\left(f_{1}, f_{2}\right)$ e $\left(y_{0}, f_{2}-f_{1}\right)$ são loose e portanto $\tilde{\omega}\left(f_{1}, f_{2}\right)=0=\tilde{\omega}\left(y_{0}, f_{2}-f_{1}\right)$. Assim, podemos supor $Y$ fechada. Se $n=2$, então $f_{1}^{*}\left(\tau_{Y}\right)$ é um 2-fibrado vetorial sobre $S^{3}$ e portanto é classificado por $\pi_{2}(S O(2)) \cong \pi_{2}\left(S^{1}\right)=0$. Assim, $f_{1}^{*}\left(\tau_{Y}\right)$ é trivial e portanto admite seção que não se anula. Agora, se $n$ é ímpar, então, pelo corolário $56, \tau_{Y}$ admite seção que não se anula o que, por conseguinte, implica que $f_{1}^{*}\left(\tau_{Y}\right)$ também admite seção que não se anula. Logo, em ambos os casos, pelo teorema $128,\left(f_{1}, f_{1}\right)$ é loose. Daí, $\tilde{\omega}\left(f_{1}, f_{1}\right)=0$ e portanto, pelo lema 134, o resultado segue.

Agora, no caso especial de raízes, a observação abaixo nos diz que os homomorfismos $\omega_{A}^{\prime}$ e $\omega_{A}^{\prime \prime}$ não dependem mais da componente conexa por caminhos $A$ de $\Omega\left(Y, y_{0}\right)$.

Observação 144. Seja $f:\left(S^{n+1}, x_{0}\right) \rightarrow\left(Y^{n}, y_{0}\right)$ uma aplicação contínua. Seja $p: \tilde{Y} \rightarrow Y$ o revestimento universal de $Y$. Agora, para $\tilde{y}_{0} \in p^{-1}\left(y_{0}\right)$, 
consideremos o (único) levantamento $\tilde{f}:\left(S^{n+1}, x_{0}\right) \rightarrow\left(\widetilde{Y}, \tilde{y}_{0}\right)$ de $f$, isto é, a (única) aplicação contínua $\tilde{f}$ tal que $\tilde{f}\left(x_{0}\right)=\tilde{y}_{0}$ e $p \circ \tilde{f}=f$.

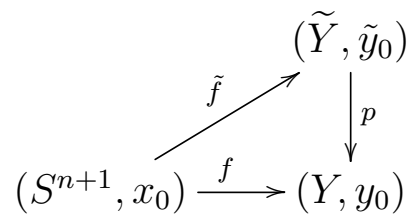

Notemos que, pelos lemas 124 e 125, a menos de isomorfismo, por abuso de notação,

$$
\tilde{\omega}\left(y_{0}, f\right) \in \Delta \subset \bigoplus_{\pi_{1}(Y)} \Omega_{1}^{f r}\left(\Omega\left(\tilde{Y}, \tilde{y}_{0}\right)\right)
$$

e as componentes são todas iguais a $\tilde{\omega}\left(\tilde{y}_{0}, \tilde{f}\right) \in \Omega_{1}^{f r}\left(\Omega\left(\widetilde{Y}, \tilde{y}_{0}\right)\right)$. Desta forma, $\omega_{A}^{\prime}\left(\left[y_{0}\right],[f]\right)=\left(r_{*} \circ p_{A} \circ \tilde{\omega}\right)\left(\left[y_{0}\right],[f]\right)=\left(r_{*} \circ p_{A}\right)\left(\tilde{\omega}\left(y_{0}, f\right)\right)=r_{*}\left(\tilde{\omega}\left(\tilde{y}_{0}, \tilde{f}\right)\right) e$ $\omega_{A}^{\prime \prime}\left(\left[y_{0}\right],[f]\right)=\left(\mu \circ p_{A} \circ \tilde{\omega}\right)\left(\left[y_{0}\right],[f]\right)=\left(\mu \circ p_{A}\right)\left(\tilde{\omega}\left(y_{0}, f\right)\right)=\mu\left(\tilde{\omega}\left(\tilde{y}_{0}, \tilde{f}\right)\right) . S e$ $\tilde{\omega}\left(\tilde{y}_{0}, \tilde{f}\right):=\left[C, \tilde{g}^{\prime}, \bar{g}\right]$, notemos que $\mu\left(\tilde{\omega}\left(\tilde{y}_{0}, \tilde{f}\right)\right)=\tilde{g}_{*}^{\prime}([C]) \in H_{1}\left(\Omega\left(\widetilde{Y}, \tilde{y}_{0}\right) ; \mathbb{Z}\right) \cong$ $\pi_{2}(Y)$ e $r_{*}\left(\tilde{\omega}\left(\tilde{y}_{0}, \tilde{f}\right)\right)=[C$, cte, $\bar{g}] \in \Omega_{1}^{f r} \cong \mathbb{Z}_{2}$.

De outro modo, se $p^{-1}\left(y_{0}\right):=\left\{\tilde{y}_{0}, \ldots, \tilde{y}_{k-1}\right\}$ e $C:=f^{-1}\left(y_{0}\right)$, notemos que $C=C_{0} \dot{\cup} \cdots \dot{\cup} C_{k-1}$, onde $C_{j}:=\tilde{f}^{-1}\left(\tilde{y}_{j}\right)$, para todo $j$. Ainda, $\Omega\left(Y, y_{0}\right)=$ $\Omega_{0} \dot{\cup} \cdots \dot{\cup} \Omega_{k-1}$, com $\Omega_{0} \approx \Omega\left(\tilde{Y}, \tilde{y}_{0}\right)$ e $\Omega_{j} \sim \Omega_{0}$, para todo $j$. Se $\tilde{\omega}\left(y_{0}, f\right):=$ $\left[C, \tilde{g}^{\prime}, \bar{g}\right] \in \Omega_{1}^{f r}\left(\Omega\left(Y, y_{0}\right)\right)$, onde $\tilde{g}^{\prime}(x)=f \circ G\left(x,{ }_{-}\right), G: C \times I \rightarrow S^{n+1}$ é tal que $G: i \simeq x_{0}$ e $i: C \hookrightarrow S^{n+1}$ é a inclusão, então $\tilde{g}^{\prime-1}\left(\Omega_{j}\right)=C_{j}$ e $\tilde{\omega}_{\Omega_{j}}\left(y_{0}, f\right)=\left[C_{j}, \tilde{g}_{\mid C_{j}}^{\prime}, \bar{g}_{\mid C_{j}}\right] . \quad$ Em particular, $\tilde{\omega}\left(\tilde{y}_{0}, \tilde{f}\right)=\left[C_{0}, \tilde{g}_{\mid C_{0}}^{\prime}, \bar{g}_{\mid C_{0}}\right] \in$ $\Omega_{1}^{f r}\left(\Omega_{0}\right), \mu\left(\tilde{\omega}\left(\tilde{y}_{0}, \tilde{f}\right)\right)=\left(\tilde{g}_{\mid C_{0}}^{\prime}\right)_{*}\left(\left[C_{0}\right]\right) \in H_{1}\left(\Omega_{0} ; \mathbb{Z}\right) \cong \pi_{2}(Y)$ e $r_{*}\left(\tilde{\omega}\left(\tilde{y}_{0}, \tilde{f}\right)\right)=$ $\left[C_{0}\right.$, cte, $\left.\bar{g}_{\mid C_{0}}\right] \in \Omega_{1}^{f r} \cong \mathbb{Z}_{2}$.

Definição 145. Com as notações da observação 144, definimos homomorfismos $\omega^{\prime}: \pi_{n+1}(Y) \rightarrow \mathbb{Z}_{2}$ e $\omega^{\prime \prime}: \pi_{n+1}(Y) \rightarrow \pi_{2}(Y)$ respectivamente por $\omega^{\prime}:[f] \mapsto r_{*}\left(\tilde{\omega}\left(\tilde{y}_{0}, \tilde{f}\right)\right) e \omega^{\prime \prime}:[f] \mapsto \mu\left(\tilde{\omega}\left(\tilde{y}_{0}, \tilde{f}\right)\right)$.

Observação 146. Observemos que, se $Y$ é simplesmente conexo, então, em particular, $\omega^{\prime}([f])=r_{*}\left(\tilde{\omega}\left(y_{0}, f\right)\right)$ e $\omega^{\prime \prime}([f])=\mu\left(\tilde{\omega}\left(y_{0}, f\right)\right)$. 
Observação 147. Notemos que, se $Y=S^{n}$, com $n \geqslant 2$, então $\Omega\left(S^{n}, y_{0}\right)$ é um $C W$-complexo conexo por caminhos tal que $\pi_{k+1}\left(S^{n}\right) \cong \pi_{k}\left(\Omega\left(S^{n}, y_{0}\right)\right)$ é abeliano finitamente gerado, para todo $k \geqslant 1$, donde, pelo lema $49, \Omega\left(S^{n}, y_{0}\right)$ tem o mesmo tipo de homotopia de um $C W$-complexo de tipo finito. Daí, pela proposição $136, \Omega_{1}^{f r}\left(\Omega\left(S^{n}, y_{0}\right)\right) \cong \mathbb{Z}_{2} \oplus \pi_{2}\left(S^{n}\right)$ e $\tilde{\omega}\left(y_{0}, f\right)$ é dado por $\omega^{\prime}([f])$ e $\omega^{\prime \prime}([f])$ (os quais calcularemos na proposição 151 e no exemplo 154). Agora, se $p: S^{n} \rightarrow Y$ é o revestimento universal de um $C W$-complexo $Y$, com $n \geqslant 2$, então $\Omega\left(Y, y_{0}\right)$ é um $C W$-complexo tal que $\pi_{k+1}\left(S^{n}\right) \cong \pi_{k+1}(Y) \cong$ $\pi_{k}\left(\Omega\left(Y, y_{0}\right)\right)$ é abeliano finitamente gerado, para todo $k \geqslant 1$. Logo, pelo lema 49, cada componente conexa por caminhos de $\Omega\left(Y, y_{0}\right)$ tem o mesmo tipo de homotopia de um $C W$-complexo de tipo finito. Daí, pela proposição 136, $\Omega_{1}^{f r}\left(\Omega\left(Y, y_{0}\right)\right) \cong\left(\underset{\pi_{1}(Y)}{\bigoplus_{Z}} \mathbb{Z}_{2}\right) \oplus\left(\bigoplus_{\pi_{1}(Y)} \pi_{2}(Y)\right)$ e $\tilde{\omega}\left(y_{0}, f\right)$ é dado por $\omega^{\prime}([f]) e$ $\omega^{\prime \prime}([f])$.

Da mesma forma, se $Y=\mathbb{C} P\left(n^{\prime}\right)$, ou $Y=\mathbb{H} P\left(n^{\prime \prime}\right)$, ou $Y$ é um $S^{a}$-fibrado sobre $S^{b}($ com $a, b \geqslant 1)$, então $\pi_{k+1}(Y) \cong \pi_{k}\left(\Omega\left(Y, y_{0}\right)\right)$ é abeliano finitamente gerado, para todo $k \geqslant 1$, usando a sequência de homotopia da fibração. Logo, pelo lema 49, cada componente conexa por caminhos de $\Omega\left(Y, y_{0}\right)$ tem o mesmo tipo de homotopia de um $C W$-complexo de tipo finito, donde podemos decompor o grupo de bordismo em soma direta e descrever $\tilde{\omega}$ em termos dos homomorfismos $\omega^{\prime}$ e $\omega^{\prime \prime}$.

Lema 148. Se $Y$ é aberta, ou $\pi_{1}(Y)$ é infinito, ou $p: Y \rightarrow B$ é uma fibração que admite uma seção, então $\omega^{\prime} \equiv 0 \equiv \omega^{\prime \prime}$.

Demonstração. De fato, pelos lemas 139, 140 e 141, segue que $\left(y_{0}, f\right)$ é loose, donde $\tilde{\omega}\left(y_{0}, f\right)=0$ e portanto $\omega^{\prime} \equiv 0 \equiv \omega^{\prime \prime}$.

Observação 149. Sobre $\omega^{\prime}$ :

Seja $f:\left(S^{n+1}, x_{0}\right) \rightarrow\left(Y^{n}, y_{0}\right)$ uma aplicação contínua. Pelo lema 148 , se 
$\pi_{1}(Y)$ é infinito, então $\omega^{\prime} \equiv 0$. Suponha que $\# \pi_{1}(Y)=k$, com $k \in \mathbb{Z}$, $k \geqslant 0$. Em particular, se $p: \widetilde{Y} \rightarrow Y$ é o revestimento universal de $Y$, então $\# p^{-1}\left(y_{0}\right)=k$. Seja $p^{-1}\left(y_{0}\right):=\left\{\tilde{y}_{0}, \ldots, \tilde{y}_{k-1}\right\}$. Seja $\tilde{f}: S^{n+1} \rightarrow \tilde{Y}$ tal que $\tilde{f}\left(x_{0}\right)=\tilde{y}_{0}$ e $p \circ \tilde{f}=f$. Consideremos uma bola aberta $B^{n} \subset \tilde{Y}$ que contém $p^{-1}\left(y_{0}\right)$ e consideremos a aplicação colapsante coll $: \widetilde{Y} \rightarrow \frac{\widetilde{Y}}{\tilde{Y} \backslash B^{n}}$.

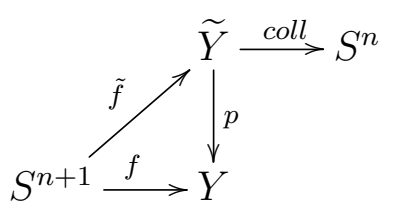

Identificando $S^{n}=\frac{\tilde{Y}}{\tilde{Y} \backslash B^{n}}$ e $\operatorname{coll}\left(\tilde{y}_{j}\right)=\tilde{y}_{j}$, obtemos que $(\operatorname{coll} \circ \tilde{f})^{-1}\left(\tilde{y}_{j}\right)=$ $\tilde{f}^{-1}\left(\tilde{y}_{j}\right)$. Daí, a menos de isomorfismo, segue que $\omega^{\prime}([f])=r_{*}\left(\tilde{\omega}\left(\tilde{y}_{0}, \tilde{f}\right)\right)=$ $\left[\tilde{f}^{-1}\left(\tilde{y}_{0}\right)\right.$, cte, $\left.\bar{g}\right]=\left[(\text { coll } \circ \tilde{f})^{-1}\left(\tilde{y}_{0}\right)\right.$, cte, $\left.\bar{g}\right] \stackrel{P T}{=} E^{\infty}([$ coll $\circ \tilde{f}])$, onde $P T: \pi_{1}^{S} \rightarrow$ $\Omega_{1}^{f r}$ denota o isomorfismo de Pontryagin-Thom (vide a observação 108).

Lema 150. Se $p: S^{n} \rightarrow Y$ é o revestimento universal de $Y$, então $\omega^{\prime}=$ $E^{\infty} \circ\left(p_{\#}\right)^{-1}: \pi_{n+1}(Y) \rightarrow \mathbb{Z}_{2}$.

Demonstração. Com efeito, se $\tilde{Y}=S^{n}$, então coll $\simeq 1_{S^{n}}$. Logo, pela observação $149, \omega^{\prime}([f])=E^{\infty}([\operatorname{coll} \circ \tilde{f}])=E^{\infty}([\tilde{f}])$, onde $\tilde{f}: S^{n+1} \rightarrow \tilde{Y}$ é tal que $\tilde{f}\left(x_{0}\right)=\tilde{y}_{0}$ e $p \circ \tilde{f}=f$. Daí, segue que $\omega^{\prime} \circ p_{\#}=E^{\infty}$. Como $n \geqslant 2$, então $p_{\#}$ é isomorfismo e portanto $\omega^{\prime}=E^{\infty} \circ\left(p_{\#}\right)^{-1}$.

\subsection{Cálculos do invariante}

Nesta seção, seja $n \geqslant 2$.

Inicialmente, estudaremos o homomorfismo $\omega^{\prime \prime}$, ora quando $Y$ é uma esfera $n$-dimensional, ora quando $Y$ é um espaço projetivo real $n$-dimensional, ora quando $Y$ é uma superfície ou uma 3-variedade.

Em seguida, estudaremos o homomorfismo $\omega^{\prime}$, nestes casos. 


\subsection{1 $\omega^{\prime \prime}$ para esferas}

Seja $Y=S^{n}$. Desde que

$$
\pi_{2}\left(S^{n}\right) \cong \begin{cases}\mathbb{Z}, & \text { se } n=2, \\ 0, & \text { caso contrário, }\end{cases}
$$

então $\omega^{\prime \prime}: \pi_{3}\left(S^{2}\right) \rightarrow \pi_{2}\left(S^{2}\right)$ é um homomorfismo de $\mathbb{Z}$ em $\mathbb{Z}$ se $n=2$, e $\omega^{\prime \prime}$ é o homomorfismo nulo, caso contrário.

Proposição 151. Se $n \geqslant 2$ e $Y=S^{n}$, então $\omega^{\prime \prime} \equiv 0$, exceto para $n=2$ quando $\omega^{\prime \prime}=\times( \pm 1)$.

Demonstração. De fato, a partir dos comentários anteriores, resta calcularmos $\omega^{\prime \prime}: \pi_{3}\left(S^{2}\right) \rightarrow \pi_{2}\left(S^{2}\right)$. Seja $h: S^{3} \rightarrow S^{2}$ a fibração de Hopf. Desde que $[h]$ gera $\pi_{3}\left(S^{2}\right)$, basta calcularmos $\omega^{\prime \prime}([h])$ para determinarmos o homomorfismo.

Primeiramente, como $S^{2}$ é simplesmente conexa, então, pela observação 146, $\omega^{\prime \prime}([h])=\mu\left(\tilde{\omega}\left(y_{0}, h\right)\right)$. Agora, notemos que $\tilde{\omega}\left(y_{0}, h\right)=\left[C, \tilde{g}^{\prime}, \bar{g}\right] \in$ $\Omega_{1}^{f r}\left(\Omega\left(S^{2}, y_{0}\right)\right)$, onde $C:=h^{-1}\left(y_{0}\right), \tilde{g}^{\prime}(x)=h \circ G\left(x,{ }_{-}\right), G: C \times I \rightarrow S^{3}$ é tal que $G: i \simeq x_{0}$ e $i: C \hookrightarrow S^{3}$ é a inclusão. Consideremos a notação dada na observação 36. Sejam $y_{0}=\left[\left(0_{\mathbb{C}}, 1,0\right)\right]$ e $h$ dada por

$$
h\left(z_{1}, z_{2}\right)= \begin{cases}S^{1} \times 0_{\mathbb{C}} & , \quad z_{2}=0, \\ {\left[\left(\frac{z_{1} \overline{z_{2}}}{\left|z_{2}\right|},\left|z_{2}\right|, 0\right)\right],} & \text { caso contrário. }\end{cases}
$$

Assim, $C=0_{\mathbb{C}} \times S^{1} \subset S^{3} \subset \mathbb{C}^{2}$. Tomando $G$ tal que $G((0,0, \cos \theta, \operatorname{sen} \theta), t)=$ $(\sqrt{2 t(1-t)(1-\cos \theta)}, 0, t+(1-t) \cos \theta,(1-t) \operatorname{sen} \theta)$, obtemos, em particular, que $\operatorname{Im} G \subset S_{+}^{2 \prime}$ (vide a observação 36). Notemos que a homotopia $G$ escolhida é tal que sua projeção no plano $0_{\mathbb{C}} \times \mathbb{C}$ é a homotopia linear dada por $t(0,0,1,0)+(1-t)(0,0, \cos \theta, \operatorname{sen} \theta)$. Seja $\bar{G}: C \rightarrow \Omega\left(S^{2}, y_{0}\right)$ dada por pr $\circ G: C \times I \rightarrow S^{2}$, onde $p r: S_{+}^{2 \prime} \rightarrow \frac{S_{+}^{2 \prime}}{0_{\mathbb{C}} \times S^{1}}$ é a aplicação quociente. 
Identificando $C$ com $S^{1}$, notemos que $[\bar{G}] \in \pi_{1}\left(\Omega\left(S^{2}, y_{0}\right)\right)$ corresponde a $\left[1_{S^{2}}\right] \in \pi_{2}\left(S^{2}\right)$. Agora, se $\bar{h}: \Omega\left(S^{2}, y_{0}\right) \rightarrow \Omega\left(S^{2}, y_{0}\right)$ é dada por $\bar{h}(\alpha)=h^{\prime \prime} \circ \alpha$ (vide a observação 36), segue que $\bar{h} \circ \bar{G}(x)(t)=h \circ G(x, t)=\tilde{g}^{\prime}(x)(t)$, donde $\bar{h} \circ \bar{G}=\tilde{g}^{\prime}$. Assim, $[\bar{h} \circ \bar{G}]=\left[\tilde{g}^{\prime}\right] \in \pi_{1}\left(\Omega\left(S^{2}, y_{0}\right)\right)$ corresponde a $\left[h^{\prime \prime} \circ 1_{S^{2}}\right]=\left[h^{\prime \prime}\right] \in \pi_{2}\left(S^{2}\right)$, via o diagrama algébrico

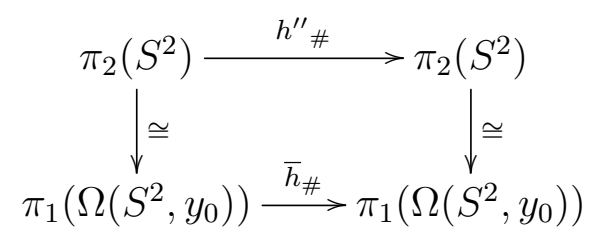

Daí, como $\mu\left(\tilde{\omega}\left(y_{0}, h\right)\right)=\tilde{g}_{*}^{\prime}([C]) \in H_{1}\left(\Omega\left(S^{2}, y_{0}\right) ; \mathbb{Z}\right)$ corresponde a $\left[\tilde{g}^{\prime}\right] \in$ $\pi_{1}\left(\Omega\left(S^{2}, y_{0}\right)\right)$ (via o isomorfismo de Hurewicz), segue que $\omega^{\prime \prime}([h])=\left[h^{\prime \prime}\right]$. Logo, uma vez que $h^{\prime \prime}$ é um homeomorfismo (vide a observação 36), então $\omega^{\prime \prime}$ é um isomorfismo (isto é, um homomorfismo de $\mathbb{Z}$ em $\mathbb{Z}$ dado pela multiplicação por \pm 1$)$.

\subsection{2 $\omega^{\prime \prime}$ para espaços projetivos reais}

Se $Y=\mathbb{R} P(n)$, então, como

$$
\pi_{2}(\mathbb{R} P(n)) \cong \pi_{2}\left(S^{n}\right) \cong \begin{cases}\mathbb{Z}, & \text { se } n=2 \\ 0, & \text { caso contrário }\end{cases}
$$

segue que $\omega^{\prime \prime}: \pi_{3}(\mathbb{R} P(2)) \rightarrow \pi_{2}(\mathbb{R} P(2))$ é um homomorfismo de $\mathbb{Z}$ em $\mathbb{Z}$ se $n=2$, e $\omega^{\prime \prime}$ é o homomorfismo nulo, caso contrário.

Proposição 152. Se $n \geqslant 2$ e $Y=\mathbb{R} P(n)$, então $\omega^{\prime \prime} \equiv 0$, exceto para $n=2$ quando $\omega^{\prime \prime}=\times( \pm 1)$.

Demonstração. De fato, a partir dos comentários anteriores, resta calcularmos $\omega^{\prime \prime}: \pi_{3}(\mathbb{R} P(2)) \rightarrow \pi_{2}(\mathbb{R} P(2))$. Seja $h: S^{3} \rightarrow S^{2}$ a fibração de Hopf. Desde que $\left[p_{\mathbb{R} P(2)} \circ h\right]$ gera $\pi_{3}(\mathbb{R} P(2))$, basta calcularmos $\omega^{\prime \prime}\left(\left[p_{\mathbb{R} P(2)} \circ h\right]\right)$ para 
determinarmos o homomorfismo. Seja $\hat{h}:=p_{\mathbb{R} P(2)} \circ h$.

Notemos que $\tilde{\omega}\left(y_{0}, \hat{h}\right)=\left[C, \tilde{g}^{\prime}, \bar{g}\right] \in \Omega_{1}^{f r}\left(\Omega\left(\mathbb{R} P(2), y_{0}\right)\right)$, onde $C:=\hat{h}^{-1}\left(y_{0}\right)$, $\tilde{g}^{\prime}(x)=\hat{h} \circ G\left(x,{ }_{-}\right), G: C \times I \rightarrow S^{3}$ é tal que $G: i \simeq x_{0}$ e $i: C \hookrightarrow S^{3}$ é a inclusão. Seja $p_{\mathbb{R} P(2)}^{-1}\left(y_{0}\right)=\left\{\tilde{y_{0}},-\tilde{y_{0}}\right\} \subset S^{2}$. Notemos que $C=h^{-1}\left(\tilde{y_{0}}\right) \dot{\cup} h^{-1}\left(-\tilde{y_{0}}\right)$. Sejam $C_{0}:=h^{-1}\left(\tilde{y}_{0}\right)$ e $C_{1}:=h^{-1}\left(-\tilde{y}_{0}\right)$. Considerando a notação dada na observação 36 , se $\tilde{y}_{0}=\left[\left(0_{\mathbb{C}}, 1,0\right)\right]$ e $h$ é dada por

$$
h\left(z_{1}, z_{2}\right)= \begin{cases}S^{1} \times 0_{\mathbb{C}} & , \quad z_{2}=0, \\ {\left[\left(\frac{z_{1} \overline{z_{2}}}{\left|z_{2}\right|},\left|z_{2}\right|, 0\right)\right],} & \text { caso contrário, }\end{cases}
$$

então $C_{0}=0_{\mathbb{C}} \times S^{1} \subset S^{3}$ e $C_{1}=S^{1} \times 0_{\mathbb{C}} \subset S^{3}$. Agora, desde que $\Omega\left(\mathbb{R} P(2), y_{0}\right)=\Omega_{0} \dot{\cup} \Omega_{1}$, onde $\alpha \in \Omega_{0}$ se, e só se, $\alpha \simeq y_{0}$, então $\tilde{g}^{\prime-1}\left(\Omega_{0}\right)=C_{0}$ e $\tilde{g}^{\prime-1}\left(\Omega_{1}\right)=C_{1}$. Assim, $\omega^{\prime \prime}([\hat{h}]) \in \pi_{2}(\mathbb{R} P(2))$ é dado por $\mu\left(\tilde{\omega}\left(\tilde{y}_{0}, h\right)\right)=$ $\mu\left(\left[C_{0}, \tilde{g}_{\mid C_{0}}^{\prime}, \bar{g}_{\mid C_{0}}\right]\right)=\left(\tilde{g}_{\mid C_{0}}^{\prime}\right)_{*}\left(\left[C_{0}\right]\right) \in H_{1}\left(\Omega_{0} ; \mathbb{Z}\right)$. De modo análogo ao que foi feito para $S^{2}$ (vide a proposição 151 ), obtemos que $\omega^{\prime \prime}([\hat{h}])=\left[p_{\mathbb{R} P(2)} \circ h^{\prime \prime}\right] \mathrm{e}$ portanto $\omega^{\prime \prime}$ é um homomorfismo de $\mathbb{Z}$ em $\mathbb{Z}$ dado pela multiplicação por \pm 1 , pois $\left[p_{\mathbb{R} P(2)} \circ h^{\prime \prime}\right]$ gera $\pi_{2}(\mathbb{R} P(2))$.

\subsection{3 $\omega^{\prime \prime}$ para superfícies e 3-variedades}

Proposição 153. Se Y é uma superfície ou uma 3-variedade, então $\omega^{\prime \prime} \equiv 0$, exceto para $Y \in\left\{S^{2}, \mathbb{R} P(2)\right\}$ quando $\omega^{\prime \prime}=\times( \pm 1)$.

Demonstração. Com efeito, se $Y$ é aberta, então, pelo lema $148, \omega^{\prime \prime} \equiv 0$. Seja $Y$ uma superfície fechada. Pelo teorema de classificação de superfícies fechadas, temos que $Y \in\left\{S^{2}, \mathbb{R} P(2), K(\pi, 1)\right\}$. Se $Y \in\left\{S^{2}, \mathbb{R} P(2)\right\}$, então, pelas observações 151 e 152, segue que $\omega^{\prime \prime}=\times( \pm 1)$. Agora, se $Y$ é $K(\pi, 1)$, então $\omega^{\prime \prime}$ é o homomorfismo nulo, visto que $\pi_{2}(Y)=\pi_{2}(K(\pi, 1))=0$.

Seja, agora, $Y$ uma 3 -variedade fechada. Seja $\widetilde{Y}$ o revestimento universal de $Y$. Temos que $Y$ ou é revestida por $S^{3}$ (que são formas espaciais esféricas), 
ou é revestida por $\mathbb{R}^{3}$ (que são $K(\pi, 1)$ ), ou é revestida por $\mathbb{R} \times S^{2}$. Nos dois primeiros casos, temos que $\omega^{\prime \prime}$ é o homomorfismo nulo, uma vez que $\pi_{2}(Y) \cong \pi_{2}(\tilde{Y})=0$. No último caso, segue que $\pi_{4}(Y) \cong \pi_{4}\left(S^{2}\right) \cong \mathbb{Z}_{2}$ e $\pi_{2}(Y) \cong \pi_{2}\left(S^{2}\right) \cong \mathbb{Z}$, donde $\omega^{\prime \prime}$ é nulo. Assim, se $Y$ é uma 3-variedade, então $\omega^{\prime \prime}: \pi_{4}(Y) \rightarrow \pi_{2}(Y)$ é o homomorfismo nulo.

\subsubsection{Sobre o homomorfismo $\omega^{\prime}$}

Nesta subseção, vamos estudar $\omega^{\prime}: \pi_{n+1}(Y) \rightarrow \mathbb{Z}_{2}$, ora quando $Y$ é uma esfera, ora quando $Y$ é um espaço projetivo real, ora quando $Y$ é uma superfície ou uma 3-variedade.

Exemplo 154. Se $Y=S^{n}$, então, pelo lema 150, temos que $\omega^{\prime}([f])=$ $E^{\infty}([f])$, isto é, $\omega^{\prime}=E^{\infty}$. Assim, se $n=2$, então

$$
\omega^{\prime}([k h])=E^{\infty}([k h])= \begin{cases}1 & \text { se } k \equiv 1(2), \\ 0 & \text { se } k \equiv 0(2),\end{cases}
$$

onde $h: S^{3} \rightarrow S^{2}$ denota a aplicação de Hopf (lembremos que [h] gera $\pi_{3}\left(S^{2}\right)$ ). Se $n>2$, então $\omega^{\prime}$ é um isomorfismo (ou seja, é a identidade de $\mathbb{Z}_{2}$ em $\mathbb{Z}_{2}$ ).

Exemplo 155. Se $Y=\mathbb{R} P(n)$, então, pelo lema 150, segue que $\omega^{\prime}([f])=$ $E^{\infty}([\tilde{f}])$, ou melhor, $\omega^{\prime}=E^{\infty} \circ\left(p_{\#}\right)^{-1}$, onde $p: S^{n} \rightarrow \mathbb{R} P(n)$ é o revestimento universal de $\mathbb{R} P(n)$ e $p \circ \tilde{f}=f$. Assim, se $n=2$, então

$$
\omega^{\prime}([k \hat{h}])=E^{\infty}([k h])= \begin{cases}1 & \text { se } k \equiv 1(2), \\ 0 & \text { se } k \equiv 0(2),\end{cases}
$$

onde $h: S^{3} \rightarrow S^{2}$ denota a aplicação de Hopf e $\hat{h}:=p \circ h$. Se $n>2$, então $\omega^{\prime}$ é um isomorfismo (ou seja, é a identidade de $\mathbb{Z}_{2}$ em $\mathbb{Z}_{2}$ ).

Exemplo 156. Seja $Y$ uma superfície ou uma 3-variedade. Se $Y$ é aberta, então, pelo lema 148, $\omega^{\prime} \equiv 0$. Seja $Y$ fechada. Se $\pi_{1}(Y)$ é infinito, então, 
novamente pelo lema $148, \omega^{\prime} \equiv 0$. Em particular, considerando os cálculos anteriores e o teorema de classificação de superfícies fechadas, encerramos a análise para superfícies. Assim, resta analisar apenas quando $Y$ é uma 3variedade fechada tal que $\pi_{1}(Y)$ é finito, isto é, quando $Y=\frac{S^{3}}{G}$ é uma 3-forma espacial esférica. Neste caso, pelo lema 150, segue que $\omega^{\prime}([f])=E^{\infty}([\tilde{f}])$, ou melhor, $\omega^{\prime}=E^{\infty} \circ\left(p_{\#}\right)^{-1}$, onde $p: S^{3} \rightarrow \frac{S^{3}}{G}$ é o revestimento universal de $\frac{S^{3}}{G}$ e $p \circ \tilde{f}=f$. Logo, $\omega^{\prime}: \pi_{4}\left(\frac{S^{3}}{G}\right) \rightarrow \mathbb{Z}_{2}$ é um isomorfismo (ou seja, é a identidade de $\mathbb{Z}_{2}$ em $\mathbb{Z}_{2}$ ).

Observação 157. Na verdade, se $Y=\frac{S^{n}}{G}$ é uma $n$-forma espacial esférica, com $n \geqslant 3$, então $\omega^{\prime}: \pi_{n+1}\left(\frac{S^{n}}{G}\right) \rightarrow \mathbb{Z}_{2}$ é um isomorfismo (ou seja, é a identidade de $\mathbb{Z}_{2}$ em $\mathbb{Z}_{2}$ ).

Observação 158. Seja $Y=S^{n}$. Então, pela observação 147, temos que $\tilde{\omega}: \pi_{n+1}\left(S^{n}\right) \rightarrow \mathbb{Z}_{2} \oplus \pi_{2}\left(S^{n}\right)$ é dado por $\tilde{\omega}=\left(\omega^{\prime}, \omega^{\prime \prime}\right)$. Daí, pelos cálculos anteriores, se $n>2$, então $\tilde{\omega}=\left(1_{\mathbb{Z}_{2}}, 0\right)$ e, se $n=2$, então $\tilde{\omega}([(2 k+1) h])=$ $(1, \pm(2 k+1))$ e $\tilde{\omega}([(2 k) h])=(0, \pm(2 k))$.

Seja $Y=\mathbb{R} P(n)$. Pela observação 147 , temos que $\tilde{\omega}: \pi_{n+1}(\mathbb{R} P(n)) \rightarrow$ $\mathbb{Z}_{2} \oplus \mathbb{Z}_{2} \oplus \pi_{2}(\mathbb{R} P(n)) \oplus \pi_{2}(\mathbb{R} P(n))$ é dado por $\tilde{\omega}=\left(\omega^{\prime}, \omega^{\prime}, \omega^{\prime \prime}, \omega^{\prime \prime}\right)$. Daí, pelos cálculos anteriores, se $n>2$, então $\tilde{\omega}=\left(1_{\mathbb{Z}_{2}}, 1_{\mathbb{Z}_{2}}, 0,0\right)$ e, se $n=2$, então $\tilde{\omega}([(2 k+1) \hat{h}])=(1,1, \pm(2 k+1), \pm(2 k+1))$ e $\tilde{\omega}([(2 k) \hat{h}])=(0,0, \pm(2 k), \pm(2 k))$.

Seja $Y$ uma superfície ou uma 3-variedade. Então $\tilde{\omega} \equiv 0$, exceto quando $Y=S^{2}$, ou $Y=\mathbb{R} P(2)$, ou $Y=\frac{S^{3}}{G}$. Para este último, pela observação 147 e pelos cálculos anteriores, temos que $\tilde{\omega}: \pi_{n+1}\left(\frac{S^{3}}{G}\right) \rightarrow\left(\bigoplus_{G} \mathbb{Z}_{2}\right) \oplus\left(\bigoplus_{G} \pi_{2}\left(\frac{S^{3}}{G}\right)\right)$ é dado por $\omega^{\prime}=1_{\mathbb{Z}_{2}}$ e $\omega^{\prime \prime} \equiv 0$. 


\subsection{Um teorema tipo Wecken}

Lembremos que, no capítulo anterior, vimos que, para codimensão 1, restava analisar a existência de um resultado tipo Wecken apenas quando $n=2,3$. Nesta seção, veremos que quando o domínio é uma esfera (isto é, $S^{3}$ ou $S^{4}$ ), então tal resultado existe.

Teorema 159. Seja $n=2,3$. Sejam $Y$ uma $n$-variedade sem bordo, conexa e $f_{1}, f_{2}: S^{n+1} \rightarrow Y$ aplicações contínuas. Então $\operatorname{MCC}\left(f_{1}, f_{2}\right)=\tilde{N}\left(f_{1}, f_{2}\right)$.

Demonstração. De fato, se $Y$ é aberta, então, pelo lema $139,\left(f_{1}, f_{2}\right)$ é loose e portanto, pela observação $120, \operatorname{MCC}\left(f_{1}, f_{2}\right)=0=\tilde{N}\left(f_{1}, f_{2}\right)$. Assim, podemos supor $Y$ fechada. Como $n=2$, ou $n=3$, então, pelo lema 143, segue que $\tilde{\omega}\left(f_{1}, f_{2}\right)=\tilde{\omega}\left(y_{0}, f_{2}-f_{1}\right)$, donde basta verificarmos o resultado para $\left(y_{0}, f_{2}-f_{1}\right)$. Denotemos $f:=f_{2}-f_{1}$.

Seja $n=2$. Pelo teorema de classificação de superfícies fechadas, temos que $Y \in\left\{K(\pi, 1), S^{2}, \mathbb{R} P(2)\right\}$. Se $Y$ é $K(\pi, 1)$, então $\pi_{1}(Y)$ é infinito e portanto, pelo lema 140 e pela observação $120, \operatorname{MCC}\left(y_{0}, f\right)=0=\tilde{N}\left(y_{0}, f\right)$. Seja $Y \in\left\{S^{2}, \mathbb{R} P(2)\right\}$. Se $Y=S^{2}$, então $f$ é uma aplicação de $S^{3}$ em $S^{2}$, isto é, $f \simeq n h$, para algum $n \in \mathbb{Z}$, onde $h: S^{3} \rightarrow S^{2}$ é a aplicação de Hopf. Se $n=0$, então $\left(y_{0}, f\right)$ é loose e portanto $M C C\left(y_{0}, f\right)=0=\tilde{N}\left(y_{0}, f\right)$. Seja $n \neq 0$. Então $\omega^{\prime \prime}([n h])=n \omega^{\prime \prime}([h])= \pm n \neq 0$ (vide a proposição 151). Portanto, como $E\left(y_{0}, f\right)$ é conexo por caminhos, segue que $\tilde{N}\left(y_{0}, f\right)=$ 1. Agora, desde que, por [K-06] (teorema 1.14), $M C C\left(y_{0}, f\right)=1$, então $M C C\left(y_{0}, f\right)=1=\tilde{N}\left(y_{0}, f\right)$. Se $Y=\mathbb{R} P(2)$, então $f$ é uma aplicação de $S^{3}$ em $\mathbb{R} P(2)$, ou seja, $f \simeq n\left(p_{\mathbb{R} P(2)} \circ h\right)$, para algum $n \in \mathbb{Z}$, onde $h: S^{3} \rightarrow S^{2}$ é a aplicação de Hopf e $p_{\mathbb{R} P(2)}$ é a aplicação quociente. Novamente, se $n=0$, então $\left(y_{0}, f\right)$ é loose e portanto $M C C\left(y_{0}, f\right)=0=\tilde{N}\left(y_{0}, f\right)$. Seja $n \neq 0$. Então $\omega^{\prime \prime}\left(\left[n\left(p_{\mathbb{R} P(2)} \circ h\right)\right]\right)= \pm n \neq 0$ (vide a proposição 152). Portanto, 
pelo lema 125 , desde que $E\left(y_{0}, f\right)$ tem duas componentes conexas por caminhos, segue que $\tilde{N}\left(y_{0}, f\right)=2$. Agora, como, por [K-08] (teorema 1.17), $M C C\left(y_{0}, f\right)=2$, então $M C C\left(y_{0}, f\right)=2=\tilde{N}\left(y_{0}, f\right)$.

Seja $n=3$. Então $Y$ ou tem grupo fundamental finito e é revestido por $S^{3}$ (que são formas espaciais esféricas), ou tem grupo fundamental infinito. Já vimos que se $\pi_{1}(Y)$ é infinito, então, pelo lema 140 e pela observação 120 , $M C C\left(y_{0}, f\right)=0=\tilde{N}\left(y_{0}, f\right)$. Seja $Y=\frac{S^{3}}{G}$ uma forma espacial esférica. Então $f$ é uma aplicação de $S^{4}$ em $\frac{S^{3}}{G}$. Como $\pi_{4}\left(\frac{S^{3}}{G}\right) \cong \pi_{4}\left(S^{3}\right) \cong \mathbb{Z}_{2}$, então $f$ é homotópica à aplicação constante, ou $f$ não é homotópica à aplicação constante. No primeiro caso, segue que $\left(y_{0}, f\right)$ é loose e portanto $M C C\left(y_{0}, f\right)=$ $0=\tilde{N}\left(y_{0}, f\right)$. No segundo caso, segue, pelo exemplo 156, que $\omega^{\prime}([f])=1$ e portanto, pelo lema 125, como $E\left(y_{0}, f\right)$ tem \#G componentes conexas por caminhos, então $\tilde{N}\left(y_{0}, f\right)=\# G$. Agora, desde que, por [K-062] (exemplo 1.25), $M C C\left(y_{0}, f\right)=\# G$, então $M C C\left(y_{0}, f\right)=\# G=\tilde{N}\left(y_{0}, f\right)$.

Corolário 160. Sejam Y uma n-variedade sem bordo, conexa e $f_{1}, f_{2}: S^{n+1} \rightarrow$ $Y$ aplicações contínuas. Então $M C C\left(f_{1}, f_{2}\right)=\tilde{N}\left(f_{1}, f_{2}\right)$.

Demonstração. Com efeito, se $n=1$ ou $n \geqslant 4$, então o resultado segue pelo corolário 123. Agora, se $n=2,3$, então o resultado segue pelo teorema 159.

O próximo corolário é uma simples aplicação do resultado tipo Wecken anterior.

Corolário 161. Sejam Y uma n-variedade sem bordo, conexa e $f_{1}, f_{2}: S^{n+1} \rightarrow$ $Y$ aplicações contínuas. Então $\left(f_{1}, f_{2}\right)$ é loose se, e só se, $\tilde{N}\left(f_{1}, f_{2}\right)=0$.

Demonstração. De fato, se $\left(f_{1}, f_{2}\right)$ é loose, então $\tilde{\omega}\left(f_{1}, f_{2}\right)=0$ e portanto $\tilde{N}\left(f_{1}, f_{2}\right)=0$. Por outro lado, se $\tilde{N}\left(f_{1}, f_{2}\right)=0$, então, como pelo resultado 
anterior $M C C\left(f_{1}, f_{2}\right)=\tilde{N}\left(f_{1}, f_{2}\right)$, segue que $M C C\left(f_{1}, f_{2}\right)=0$, ou seja, $\left(f_{1}, f_{2}\right)$ é loose.

\subsection{Mais cálculos de $\omega^{\prime \prime}$}

Nesta seção, sejam $n \geqslant 2$ e $Y$ uma $n$-variedade fechada, conexa. Já analisa$\operatorname{mos} \omega^{\prime \prime}: \pi_{n+1}(Y) \rightarrow \pi_{2}(Y)$ quando $Y$ é uma esfera, um espaço projetivo real, uma superfície e uma 3-variedade. Estudaremos, agora, o homomorfismo $\omega^{\prime \prime}$, para vários espaços $Y$, a saber, quando $Y$ é um espaço projetivo complexo, quaterniônico, um fibrado esférico sobre uma esfera e uma variedade de Stiefel (real). Inicialmente, faremos algumas considerações gerais envolvendo espaços $K(\pi, 1)$ e o revestimento universal de $Y$.

Observação 162. Considerações envolvendo espaços $K(\pi, 1)$ :

Se $Y$ é $K(\pi, 1)$, então claramente $\omega^{\prime \prime}: \pi_{n+1}(Y) \rightarrow \pi_{2}(Y)$ é o homomorfismo nulo. Em particular, se $Y$ é um $K(\pi, 1)$-fibrado sobre $K(\pi, 1)$, segue que $\omega^{\prime \prime} \equiv 0$. Seja $M$ uma variedade. Então, por um lado, se $Y$ é um $M$-fibrado sobre $K(\pi, 1)$, segue que $\pi_{i}(Y) \cong \pi_{i}(M)$, para todo $i \geqslant 2$ e portanto $\omega^{\prime \prime}$ é um homomorfismo de $\pi_{n+1}(M)$ em $\pi_{2}(M)$. Por outro lado, se $Y$ é um $K(\pi, 1)$ fibrado sobre $M$, então $\pi_{n+1}(Y) \cong \pi_{n+1}(M)$ e $\pi_{2}(Y) \cong \operatorname{ker}\left(\pi_{2}(M) \rightarrow \pi\right)$, donde $\omega^{\prime \prime}$ é um homomorfismo de $\pi_{n+1}(M)$ em $\operatorname{ker}\left(\pi_{2}(M) \rightarrow \pi\right)$.

Observação 163. Considerações envolvendo o revestimento universal de $Y$ : Se $\widetilde{Y}$ é o revestimento universal de $Y$, então $\pi_{i}(Y) \cong \pi_{i}(\widetilde{Y})$, para todo $i \geqslant 2$ e, em particular, pelo teorema de Hurewicz, obtemos que $\pi_{2}(Y) \cong \pi_{2}(\widetilde{Y}) \cong$ $H_{2}(\widetilde{Y} ; \mathbb{Z})$ (pois $\widetilde{Y}$ é simplesmente conexo), donde $\omega^{\prime \prime}$ é um homomorfismo de $\pi_{n+1}(\tilde{Y})$ em $H_{2}(\tilde{Y} ; \mathbb{Z})$. Em particular, se $Y$ é simplesmente conexo, então $\omega^{\prime \prime}$ é um homomorfismo de $\pi_{n+1}(Y)$ em $H_{2}(Y ; \mathbb{Z})$. Se $Y$ é 2-conexo, então claramente $\omega^{\prime \prime}=0$. 


\subsubsection{Espaços projetivos complexos e quaterniônicos}

Seja $n=2 n^{\prime}$. Se $Y=\mathbb{C} P\left(n^{\prime}\right)$, então $\pi_{n+1}\left(\mathbb{C} P\left(n^{\prime}\right)\right) \cong \pi_{n+1}\left(S^{n+1}\right) \cong \mathbb{Z}$ e $\pi_{2}\left(\mathbb{C} P\left(n^{\prime}\right)\right) \cong \pi_{1}\left(S^{1}\right) \cong \mathbb{Z}$, pois $\left(S^{n+1}, p, \mathbb{C} P\left(n^{\prime}\right), S^{1}\right)$ é um $S^{1}$-fibrado sobre $\mathbb{C} P\left(n^{\prime}\right)$ (vide o exemplo 35). Logo, $\omega^{\prime \prime}: \pi_{n+1}\left(\mathbb{C} P\left(n^{\prime}\right)\right) \rightarrow \pi_{2}\left(\mathbb{C} P\left(n^{\prime}\right)\right)$ é um homomorfismo de $\mathbb{Z}$ em $\mathbb{Z}$.

Proposição 164. Se $Y=\mathbb{C} P\left(n^{\prime}\right)$, então $\omega^{\prime \prime}=\times( \pm 1)$, para todo $n^{\prime} \geqslant 1$.

Demonstração. Com efeito, sejam $n=2 n^{\prime}$ e $p: S^{n+1} \rightarrow \mathbb{C} P\left(n^{\prime}\right)$ a aplicação de Hopf. Desde que $[p]$ gera $\pi_{n+1}\left(\mathbb{C} P\left(n^{\prime}\right)\right)$, basta calcularmos $\omega^{\prime \prime}([p])$ para determinarmos o homomorfismo.

Inicialmente, como $\mathbb{C} P\left(n^{\prime}\right)$ é simplesmente conexo, então, pela observação 146, $\omega^{\prime \prime}([p])=\mu\left(\tilde{\omega}\left(y_{0}, p\right)\right)$. Agora, notemos que $\tilde{\omega}\left(y_{0}, p\right)=\left[C, \tilde{g}^{\prime}, \bar{g}\right] \in$ $\Omega_{1}^{f r}\left(\Omega\left(\mathbb{C} P\left(n^{\prime}\right), y_{0}\right)\right)$, onde $C:=p^{-1}\left(y_{0}\right), \tilde{g}^{\prime}(x)=p \circ G\left(x,{ }_{-}\right), G: C \times I \rightarrow S^{n+1}$ é tal que $G: i \simeq x_{0}$ e $i: C \hookrightarrow S^{n+1}$ é a inclusão. Consideremos a notação dada na observação 37 . Sejam $y_{0}=\left[\left(0_{\mathbb{C}}, 1,0,0_{\mathbb{C}}, \ldots, 0_{\mathbb{C}}\right)\right]$ e $p$ dada por

$$
p\left(z_{1}, \ldots, z_{n^{\prime}+1}\right)= \begin{cases}S^{1} \times 0_{\mathbb{C}} \times \cdots \times 0_{\mathbb{C}} & , z_{2}=\cdots=z_{n^{\prime}+1}=0, \\ {\left[\left(\frac{z_{1} \overline{z_{2}}}{\left|z_{2}\right|},\left|z_{2}\right|, 0, \frac{z_{3} \overline{z_{2}}}{\left|z_{2}\right|}, \ldots, \frac{z_{n^{\prime}+1} \overline{z_{2}}}{\left|z_{2}\right|}\right)\right]} & , z_{2} \neq 0, \\ \vdots & \\ {\left[\left(\frac{z_{1} \overline{z_{n^{\prime}+1}}}{\left|z_{n^{\prime}+1}\right|}, \frac{z_{2} \overline{z_{n^{\prime}+1}}}{\left|z_{n^{\prime}+1}\right|}, \ldots, \frac{z_{n^{\prime}} \overline{z_{n^{\prime}+1}}}{\left|z_{n^{\prime}+1}\right|},\left|z_{n^{\prime}+1}\right|, 0\right)\right], z_{n^{\prime}+1} \neq 0 .}\end{cases}
$$

Assim, $C=0_{\mathbb{C}} \times S^{1} \times 0_{\mathbb{C}} \times \cdots \times 0_{\mathbb{C}} \subset S^{n+1}$. Analogamente ao que foi feito para $S^{2}$ (vide a proposição 151 ), escolhendo $G$ adequada e identificando $C$ com $S^{1}$, obtemos $\bar{G}: C \rightarrow \Omega\left(S^{2}, y_{0}\right)$ tal que $[\bar{G}] \in \pi_{1}\left(\Omega\left(S^{2}, y_{0}\right)\right)$ corresponde a $\left[1_{S^{2}}\right] \in \pi_{2}\left(S^{2}\right)$. Daí, $[\bar{p} \circ \bar{G}]=\left[\tilde{g}^{\prime}\right] \in \pi_{1}\left(\Omega\left(\mathbb{C} P\left(n^{\prime}\right), y_{0}\right)\right)$ corresponde a $\left[j \circ p^{\prime \prime} \circ 1_{S^{2}}\right]=\left[j \circ p^{\prime \prime}\right] \in \pi_{2}\left(\mathbb{C} P\left(n^{\prime}\right)\right)$, onde $j: S^{2} \hookrightarrow \mathbb{C} P\left(n^{\prime}\right)$ denota a inclusão e $\bar{p}: \Omega\left(S^{2}, y_{0}\right) \rightarrow \Omega\left(\mathbb{C} P\left(n^{\prime}\right), y_{0}\right)$ é dada por $\bar{p}(\alpha)=j \circ p^{\prime \prime} \circ \alpha$ (vide a observação 37$)$. Logo, como $\mu\left(\tilde{\omega}\left(y_{0}, p\right)\right)=\tilde{g}_{*}^{\prime}([C]) \in H_{1}\left(\Omega\left(\mathbb{C} P\left(n^{\prime}\right), y_{0}\right) ; \mathbb{Z}\right)$ corresponde a $\left[\tilde{g}^{\prime}\right] \in \pi_{1}\left(\Omega\left(\mathbb{C} P\left(n^{\prime}\right), y_{0}\right)\right)$ (via o isomorfismo de Hurewicz), segue 
que $\omega^{\prime \prime}([p])=\left[j \circ p^{\prime \prime}\right]$. Daí, desde que $p^{\prime \prime}$ é um homeomorfismo (vide a observação 37) e $j_{\#}: \pi_{2}\left(S^{2}\right) \rightarrow \pi_{2}\left(\mathbb{C} P\left(n^{\prime}\right)\right)$ é um isomorfismo, segue que $\omega^{\prime \prime}$ é um isomorfismo (isto é, um homomorfismo de $\mathbb{Z}$ em $\mathbb{Z}$ dado pela multiplicação por \pm 1$)$.

Seja agora $n=4 n^{\prime \prime}$. Se $Y=\mathbb{H} P\left(n^{\prime \prime}\right)$, então $\omega^{\prime \prime}$ é o homomorfismo nulo, uma vez que $\pi_{2}\left(\mathbb{H} P\left(n^{\prime \prime}\right)\right)=0$, pois $\left(S^{n+1}, p^{\prime}, \mathbb{H} P\left(n^{\prime \prime}\right), S^{3}\right)$ é $S^{3}$-fibrado sobre $\mathbb{H} P\left(n^{\prime \prime}\right)$ (vide o exemplo 35$)$.

Proposição 165. Se $Y=\mathbb{H} P\left(n^{\prime \prime}\right)$, então $\omega^{\prime \prime}=0$, para todo $n^{\prime \prime} \geqslant 1$.

Demonstração. Segue do comentário anterior.

\subsubsection{Fibrados esféricos sobre esferas}

Sejam $a, b \in \mathbb{Z}, a, b \geqslant 1$. Seja $p: Y \rightarrow S^{b}$ um $S^{a}$-fibrado sobre $S^{b}$. Em particular, $n=a+b$ e $Y$ é uma variedade $(a+b)$-dimensional. Desta forma, estamos interessados em estudar $\omega^{\prime \prime}: \pi_{a+b+1}(Y) \rightarrow \pi_{2}(Y)$.

Proposição 166. Seja $p: Y \rightarrow S^{b}$ um $S^{a}$-fibrado sobre $S^{b}$, com $a, b \in \mathbb{Z}$, $a, b \geqslant 1$. Se $(a, b) \neq(2,4)$, então $\omega^{\prime \prime} \equiv 0$.

Demonstração. De fato, se $a \geqslant b$, então $S^{a}$ é $(b-1)$-conexa e portanto, por [Hu-94] (página 21), p admite uma seção. Daí, pelo lema 148, $\omega^{\prime \prime} \equiv 0$. Seja, agora, $a<b$. Consideremos a sequência de homotopia da fibração $p$.

$$
\begin{gathered}
\cdots \pi_{a+b+1}\left(S^{a}\right) \rightarrow \pi_{a+b+1}(Y) \rightarrow \pi_{a+b+1}\left(S^{b}\right) \rightarrow \pi_{a+b}\left(S^{a}\right) \rightarrow \cdots \\
\cdots \rightarrow \pi_{3}\left(S^{b}\right) \rightarrow \pi_{2}\left(S^{a}\right) \rightarrow \pi_{2}(Y) \rightarrow \pi_{2}\left(S^{b}\right) \cdots
\end{gathered}
$$

Se $b>a \geqslant 3$, ou $b \geqslant 3$ e $a=1$, então $\pi_{2}\left(S^{a}\right)=0=\pi_{2}\left(S^{b}\right)$ donde $\pi_{2}(Y)=0$ e portanto $\omega^{\prime \prime} \equiv 0$. Se $b=2$ e $a=1$, então, como $0=\pi_{2}\left(S^{1}\right) \rightarrow \pi_{2}(Y) \rightarrow$ $\pi_{2}\left(S^{2}\right) \cong \mathbb{Z}$ e $0=\pi_{4}\left(S^{1}\right) \rightarrow \pi_{4}(Y) \rightarrow \pi_{4}\left(S^{2}\right) \rightarrow \pi_{3}\left(S^{1}\right)=0$ são exatas, 
segue que $\pi_{2}(Y) \leqslant \mathbb{Z}$ e $\pi_{4}(Y) \cong \pi_{4}\left(S^{2}\right) \cong \mathbb{Z}_{2}$ e portanto $\omega^{\prime \prime} \equiv 0$. Sejam $a=2$ e $b=3$. Como, por [Sm-59], $\operatorname{Diff}\left(S^{2}\right) \sim O(3)$, então $\pi_{2}\left(\operatorname{Diff}\left(S^{2}\right)\right) \cong$ $\pi_{2}(O(3))=\pi_{2}(S O(3)) \cong \pi_{2}(\mathbb{R} P(3)) \cong \pi_{2}\left(S^{3}\right)=0$. Daí, $Y \approx S^{3} \times S^{2}$ e portanto, pelo lema $148, \omega^{\prime \prime} \equiv 0$. Finalmente, sejam $a=2$ e $b \geqslant 4$. Desde que $0=\pi_{3}\left(S^{b}\right) \rightarrow \pi_{2}\left(S^{2}\right) \rightarrow \pi_{2}(Y) \rightarrow \pi_{2}\left(S^{b}\right)=0$ é exata, então $\pi_{2}(Y) \cong \pi_{2}\left(S^{2}\right) \cong \mathbb{Z}$. Ainda, como $\pi_{b+3}\left(S^{2}\right) \rightarrow \pi_{b+3}(Y) \rightarrow \pi_{b+3}\left(S^{b}\right)$ é exata e, por [Se-51], $\pi_{b+3}\left(S^{2}\right)$ é finito (pois $b \geqslant 4$ ) e $\pi_{b+3}\left(S^{b}\right)$ é infinito se, e só se, $b=4$, segue que $\omega^{\prime \prime} \equiv 0$ quando $a=2$ e $b \geqslant 5$.

Assim, resta estudar $\omega^{\prime \prime}$ quando $Y$ é um $S^{2}$-fibrado sobre $S^{4}$. Nesta situação, $\omega^{\prime \prime}$ é um homomorfismo de $\pi_{7}(Y)$ em $\pi_{2}(Y)$. Observemos que, da sequência de homotopia da fibração $p$, temos que $\pi_{2}(Y) \cong \mathbb{Z}$ e $\pi_{7}(Y)$ é tal que a sequência $\mathbb{Z}_{2} \oplus \mathbb{Z}_{2} \rightarrow \mathbb{Z}_{2} \rightarrow \pi_{7}(Y) \rightarrow \mathbb{Z} \oplus \mathbb{Z}_{12} \rightarrow \mathbb{Z}_{12}$ é exata, donde, em particular, $\pi_{7}(Y) \cong \mathbb{Z} \oplus A$, para algum grupo abeliano finito $A$.

Se $Y \approx S^{4} \times S^{2}$, então, pelo lema $148, \omega^{\prime \prime} \equiv 0$. Seja $p: Y \rightarrow S^{4}$ um $S^{2}$-fibrado não trivial sobre $S^{4}$. Da sequência de homotopia da fibração $p$, consideremos o homomorfismo conectante $\partial: \pi_{4}\left(S^{4}\right) \rightarrow \pi_{3}\left(S^{2}\right)$. Notemos que $\partial$ é um homomorfismo de $\mathbb{Z}$ em $\mathbb{Z}$ e que, a cada fibrado $Y$, está associado um número inteiro $\partial(1) \in \mathbb{Z}$. Para maiores detalhes, vide [JaWhj-54] e [JaWhj-55].

Inicialmente, construiremos um $S^{2}$-fibrado sobre $S^{4}$ tal que $\partial(1)=1$ e estudaremos o homomorfismo $\omega^{\prime \prime}$ para este fibrado. Em seguida, construiremos, para cada inteiro $k$, um $S^{2}$-fibrado sobre $S^{4} \operatorname{com} \partial(1)=k$ e calcularemos $\omega^{\prime \prime}$ para cada um destes fibrados.

Caso I - $\partial(\mathbf{1})=\mathbf{1}$.

Consideremos a fibração de Hopf, $g_{4}$, sobre $\mathbb{H} P(1) \approx S^{4}$. Notemos que $g_{4}$ é um $S^{3}$-fibrado principal sobre $S^{4}$ e que $S^{1}$ atua por restrição no espaço total 
$S^{7}$ e na fibra $S^{3}$ deste fibrado. Daí, considerando o quociente por esta ação, obtemos um $S^{2}$-fibrado sobre $S^{4}, p: \mathbb{C} P(3) \rightarrow S^{4}$, conforme o diagrama geométrico abaixo

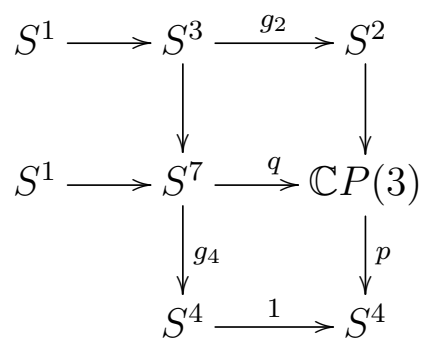

onde $g_{2}$ é a fibração de Hopf sobre $\mathbb{C} P(1) \approx S^{2}$ e $q$ é a fibração de Hopf sobre $\mathbb{C} P(3)$. Como $S^{1}$ é $K(\mathbb{Z}, 1)$, então, da sequência de homotopia da fibração $q$, obtemos que $\pi_{i}\left(S^{7}\right) \stackrel{q_{\#}}{\rightarrow} \pi_{i}(\mathbb{C} P(3))$ é isomorfismo, para todo $i \geqslant 3$, $\pi_{2}(\mathbb{C} P(3)) \cong \pi_{1}\left(S^{1}\right) \cong \mathbb{Z}$ e $\pi_{1}\left(\mathbb{C} P^{3}\right)=1$. Daí, da sequência de homotopia da fibração $p$, segue que o homomorfismo conectante $\partial: \pi_{4}\left(S^{4}\right) \rightarrow \pi_{3}\left(S^{2}\right)$ é um isomorfismo de $\mathbb{Z}$ em $\mathbb{Z}$. Sem perda de generalidade, seja $\partial(1)=1$. Desta forma, $\mathbb{C} P(3)$ é um $S^{2}$-fibrado sobre $S^{4}$ tal que $\partial(1)=1$. Finalmente, notemos que já analisamos $\omega^{\prime \prime}$ para $\mathbb{C} P(3)$, a saber, pela proposição 164, segue que $\omega^{\prime \prime}: \pi_{7}(\mathbb{C} P(3)) \rightarrow \pi_{2}(\mathbb{C} P(3))$ é um isomorfismo de $\mathbb{Z}$ em $\mathbb{Z}$, isto é, $\omega^{\prime \prime}= \pm 1$.

Vamos, agora, fixar algumas notações referentes a este caso, que serão utilizadas posteriormente. Da sequência de homotopia da fibração $g_{2}$, segue que $\pi_{i}\left(S^{3}\right) \stackrel{g_{2} \#}{\rightarrow} \pi_{i}\left(S^{2}\right)$ é isomorfismo, para todo $i \geqslant 3$. Consideremos, agora, as sequências de homotopia das fibrações $g_{4}$ e $p$, e o seguinte diagrama algébrico decorrente do diagrama geométrico acima:

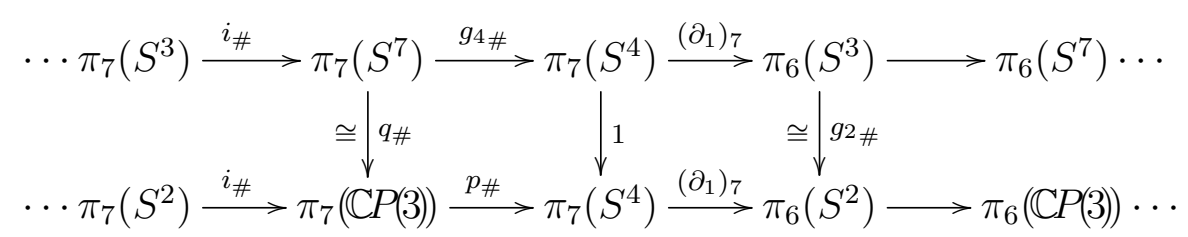

Desde que $\pi_{6}(\mathbb{C} P(3)) \cong \pi_{6}\left(S^{7}\right)=0$, então ambos os homomorfismos 
conectantes $\left(\partial_{1}\right)_{7}$ são sobrejetores. Ainda, como $\pi_{7}\left(S^{2}\right) \cong \pi_{7}\left(S^{3}\right) \cong \mathbb{Z}_{2}$ finito e $\pi_{7}(\mathbb{C} P(3)) \cong \pi_{7}\left(S^{7}\right) \cong \mathbb{Z}$, segue que ambos os homomorfismos $i_{\#}$ são nulos, donde obtemos o diagrama algébrico abaixo.

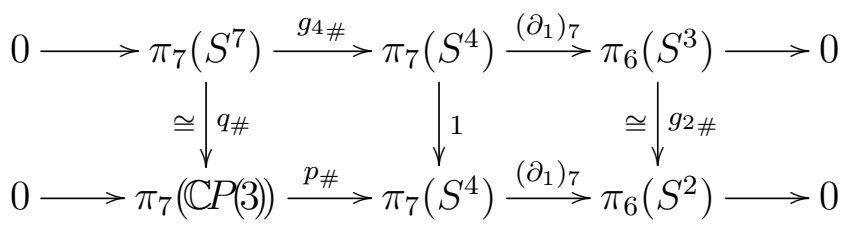

Desde que $g_{4 \#}\left(\iota_{7}\right)=\left[g_{4}\right]$ e $q_{\#}\left(\iota_{7}\right)=[q]$, onde $\iota_{7} \in \pi_{7}\left(S^{7}\right)$ denota a classe de homotopia da identidade de $S^{7}$, então $p_{\#}([q])=\left[g_{4}\right]$.

Caso II - $\partial(\mathbf{1})=\mathbf{k} \in \mathbb{Z}$.

Sejam $\xi_{1}:=\left(\mathbb{C} P(3), p, S^{4}, S^{2}\right)$ e $f_{k}: S^{4} \rightarrow S^{4}$ uma aplicação contínua de grau $k$, com $k \in \mathbb{Z}$. Denotemos o pullback de $\xi_{1}$ por $f_{k}$ simplesmente por $\xi_{k}:=\left(Y_{k}, p_{k}, S^{4}, S^{2}\right)$. Consideremos o seguinte diagrama geométrico

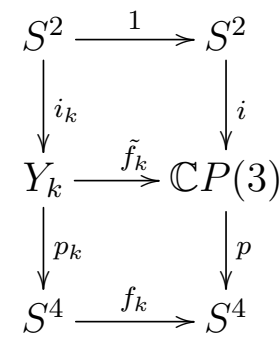

onde $i, i_{k}$ são as respectivas inclusões. Agora, denotando os homomorfismos conectantes de $Y_{k}$ por $\left(\partial_{k}\right)_{j}: \pi_{j}\left(S^{4}\right) \rightarrow \pi_{j-1}\left(S^{2}\right)$, com $j \geqslant 2$, consideremos, a partir do diagrama geométrico acima e das sequências de homotopia das fibrações $p_{k}$ e $p$, o diagrama algébrico abaixo.

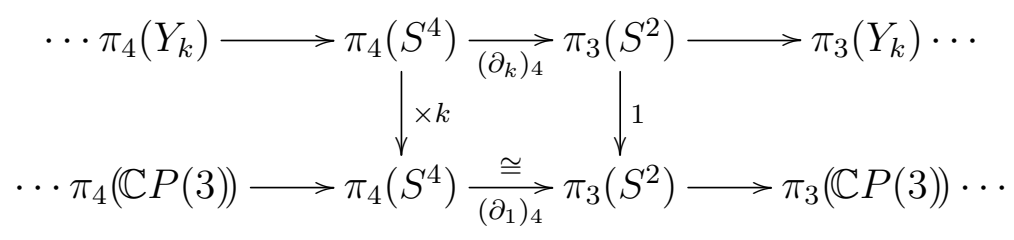

Daí, como, pelo caso anterior, $\left(\partial_{1}\right)_{4}(1)=1$, então $\left(\partial_{k}\right)_{4}(1)=k$. Desta 
forma, $Y_{k}$ é um $S^{2}$-fibrado sobre $S^{4}$ tal que $\partial(1)=k \in \mathbb{Z}$. Em particular, se $k, l \in \mathbb{Z}$, com $k \neq l$, então $Y_{k} \not \approx Y_{l}$.

O resultado abaixo nos ajuda a calcular $\left(\omega^{\prime \prime}\right)_{k}$ a partir do cálculo de $\left(\omega^{\prime \prime}\right)_{1}$, onde $\left(\omega^{\prime \prime}\right)_{k}$ denota $\omega^{\prime \prime}$ para $Y_{k}$ e $\left(\omega^{\prime \prime}\right)_{1}$ denota $\omega^{\prime \prime}$ para $\mathbb{C} P(3)$.

Proposição 167. Com as notações acima, o diagrama algébrico

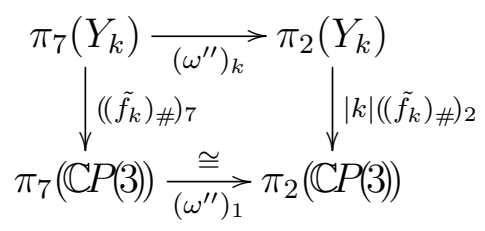

é comutativo, onde $|k|\left(\left(\tilde{f}_{k}\right)_{\#}\right)_{2}=\times( \pm|k|): \mathbb{Z} \rightarrow \mathbb{Z} e\left(\omega^{\prime \prime}\right)_{1}= \pm 1: \mathbb{Z} \rightarrow \mathbb{Z}$.

Demonstração. De fato, temos, pela proposição 164 , que $\left(\omega^{\prime \prime}\right)_{1}$ é um isomorfismo de $\mathbb{Z}$ em $\mathbb{Z}$, ou seja, $\left(\omega^{\prime \prime}\right)_{1}= \pm 1$. Agora, do diagrama algébrico abaixo, decorrente das sequências de homotopia das fibrações $p_{k}$ e $p$ e do diagrama geométrico 69.1 , que relaciona estas fibrações, notemos que $\left(\left(\tilde{f}_{k}\right)_{\#}\right)_{2}$ também é um isomorfismo de $\mathbb{Z}$ em $\mathbb{Z}$

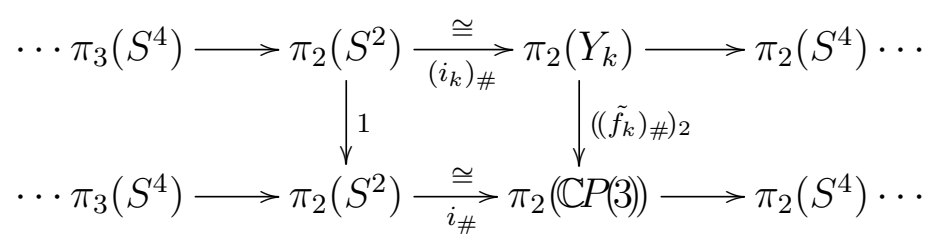

pois $\pi_{3}\left(S^{4}\right)=0$ e $\pi_{2}\left(S^{4}\right)=0$. Em particular, $|k|\left(\left(\tilde{f}_{k}\right)_{\#}\right)_{2}=|k|( \pm 1)=$ $\times( \pm|k|)$. Vamos, agora, verificar a comutatividade do diagrama do enunciado. Se $k=0$, então $\left(\left(\tilde{f}_{0}\right)_{\#}\right)_{7}$ é o homomorfismo nulo e portanto o diagrama do enunciado é comutativo. Sem perda de generalidade, seja $k>0$. Seja $b_{0} \in S^{4}$. Sejam $B_{1}, \ldots, B_{k}$ bolas abertas em $S^{4}$ disjuntas tais que $f_{k}\left(S^{4} \backslash \underset{j}{\dot{\cup}} B_{j}\right) \equiv-b_{0}$ e $f_{k \mid B_{j}}: B_{j} \rightarrow S^{4} \backslash\left\{-b_{0}\right\}$ é um homeomorfismo. Desta forma, $f_{k}^{-1}\left(b_{0}\right):=\left\{b_{1}, \ldots, b_{k}\right\}$, sendo $b_{j}$ o centro de $B_{j}$. Seja $y_{0} \in \mathbb{C} P(3)$ tal que $p\left(y_{0}\right)=b_{0}$. Então $\tilde{f}_{k}^{-1}\left(y_{0}\right):=\left\{y_{1}, \ldots, y_{k}\right\}$, onde $p_{k}\left(y_{j}\right)=b_{j}$. Seja 
$u: S^{7} \rightarrow Y_{k}$ uma aplicação contínua. Temos que $\left(\tilde{f}_{k} \circ u\right)^{-1}\left(y_{0}\right)=\underset{j}{\cup} u^{-1}\left(y_{j}\right)$. Consideremos agora o diagrama geométrico abaixo.

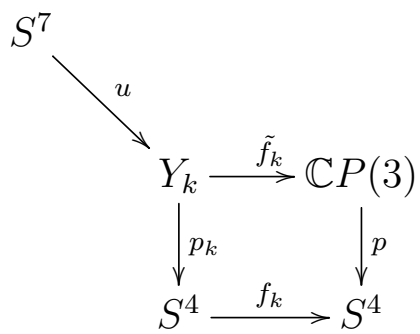

Consideremos $y_{1}$ como sendo o ponto base de $Y_{k}$. Desde que $Y_{k}$ é simplesmente conexo, então, pela observação $146,\left(\omega^{\prime \prime}\right)_{k}([u])=\mu\left(\tilde{\omega}\left(y_{1}, u\right)\right)$. Agora, temos que $\tilde{\omega}\left(y_{1}, u\right)=\left[C, \tilde{g}^{\prime}, \bar{g}\right] \in \Omega_{1}^{f r}\left(\Omega\left(Y_{k}, y_{1}\right)\right)$, onde $C:=u^{-1}\left(y_{1}\right), \tilde{g}^{\prime}(x)=$ $u \circ G\left(x,{ }_{-}\right), G: C \times I \rightarrow S^{7}$ é tal que $G: i \simeq$ cte e $i: C \hookrightarrow S^{7}$ é a inclusão. Como $Y_{k}$ é uma 6-variedade tal que $\pi_{1}\left(Y_{k}\right)=1$, então, pelo teorema 116, segue que $M C C\left(y_{1}, u\right) \leqslant R\left(y_{1}, u\right)=1$ e portanto podemos supor que $C$ é conexo por caminhos. Identificando $C$ com $S^{1}$, segue que $\left[\tilde{g}^{\prime}\right] \in \pi_{1}\left(\Omega\left(Y_{k}, y_{1}\right)\right)$ e, identificando $\pi_{1}\left(\Omega\left(Y_{k}, y_{1}\right)\right) \cong \pi_{2}\left(Y_{k}\right)$, obtemos, a menos de isomorfismo, que $\left(\omega^{\prime \prime}\right)_{k}([u])=\left[\tilde{g}^{\prime}\right]$. Da mesma forma, temos que $\tilde{\omega}\left(y_{0}, \tilde{f}_{k} \circ u\right)=\left[D, \tilde{h}^{\prime}, \bar{h}\right] \in \Omega_{1}^{f r}\left(\Omega\left(\mathbb{C} P(3), y_{0}\right)\right)$, onde $D:=\left(\tilde{f}_{k} \circ u\right)^{-1}\left(y_{0}\right)$, $\tilde{h}^{\prime}(x)=\left(\tilde{f}_{k} \circ u\right) \circ H\left(x,{ }_{-}\right), H: D \times I \rightarrow S^{7}$ é tal que $H: i \simeq$ cte e $i: D \hookrightarrow S^{7}$ é a inclusão. Em particular, $\bar{h}_{\mid C}=\bar{g}$ e tomando $H$ como sendo tal que $H_{\mid C \times I}=G$, segue que $\tilde{h}_{\mid C}^{\prime}=\tilde{f}_{k} \circ \tilde{g}^{\prime}$. Desde que $u^{-1}\left(y_{j}\right)$ e $C$ são cobordantes, com $1 \leqslant j \leqslant k$, então podemos tomar $D=\underset{j}{\dot{u} C}$. Desta forma, $\left[D, \tilde{h}^{\prime}, \bar{h}\right]=k\left[C, \tilde{h}_{\mid C}^{\prime}, \bar{h}_{\mid C}\right]=k\left[C, \tilde{f}_{k} \circ \tilde{g}^{\prime}, \bar{g}\right] \mathrm{e}$ 


$$
\begin{aligned}
\left(\omega^{\prime \prime}\right)_{1}\left(\left(\left(\tilde{f}_{k}\right)_{\#}\right)_{7}([u])\right) & =\left(\omega^{\prime \prime}\right)_{1}\left(\left[\tilde{f}_{k} \circ u\right]\right) \\
& =\mu\left(\tilde{\omega}\left(y_{0}, \tilde{f}_{k} \circ u\right)\right) \\
& =\mu\left(\left[D, \tilde{h}^{\prime}, \bar{h}\right]\right) \\
& =k \mu\left(\left[C, \tilde{f}_{k} \circ \tilde{g}^{\prime}, \bar{g}\right]\right) \\
& =k\left[\tilde{f}_{k} \circ \tilde{g}^{\prime}\right] \\
& =k\left(\left(\tilde{f}_{k}\right)_{\#}\right)_{2}\left(\left[\tilde{g}^{\prime}\right]\right) \\
& =k\left(\left(\tilde{f}_{k}\right)_{\#}\right)_{2}\left(\left(\omega^{\prime \prime}\right)_{k}([u])\right)
\end{aligned}
$$

Portanto, $k\left(\left(\tilde{f}_{k}\right)_{\#}\right)_{2} \circ\left(\omega^{\prime \prime}\right)_{k}=\left(\omega^{\prime \prime}\right)_{1} \circ\left(\left(\tilde{f}_{k}\right)_{\#}\right)_{7}$. Se $k<0$, obtemos que $\left[D, \tilde{h}^{\prime}, \bar{h}\right]=(-k)\left[C, \tilde{f}_{k} \circ \tilde{g}^{\prime}, \bar{g}\right]$ e $(-k)\left(\left(\tilde{f}_{k}\right)_{\#}\right)_{2} \circ\left(\omega^{\prime \prime}\right)_{k}=\left(\omega^{\prime \prime}\right)_{1} \circ\left(\left(\tilde{f}_{k}\right)_{\#}\right)_{7}$, donde o resultado segue.

Assim, a fim de calcular $\omega^{\prime \prime}$ para $Y_{k}$, determinemos primeiramente o homomorfismo $\left(\left(\tilde{f}_{k}\right)_{\#}\right)_{7}: \pi_{7}\left(Y_{k}\right) \rightarrow \pi_{7}(\mathbb{C} P(3))$. Lembremos que $\pi_{7}\left(Y_{k}\right) \cong \mathbb{Z} \oplus A$, para algum grupo abeliano finito $A$. Consideremos o diagrama algébrico abaixo, dado pelo resultado anterior.

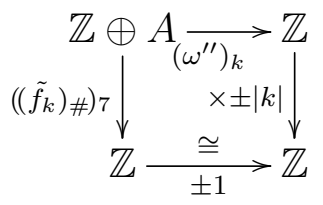

Notemos que, os homomorfismos $\left(\left(\tilde{f}_{k}\right)_{\#}\right)_{7}$ e $\left(\omega^{\prime \prime}\right)_{k}$ restritos a $A$ são nulos, pois $\operatorname{Hom}(A, \mathbb{Z})=0$. Assim, independemente de conhecermos $\pi_{7}\left(Y_{k}\right)$, basta calcularmos $\left(\left(\tilde{f}_{k}\right)_{\#}\right)_{7}$ nos elementos da forma $(n, 0)$, a fim de determinar $\omega^{\prime \prime}$ para $Y_{k}$. Ao final da subseção, vamos, também, determinar $\pi_{7}\left(Y_{k}\right)$ por ser uma questão interessante por si própria.

Proposição 168. Seja $k \in \mathbb{Z}$. Se $\pi_{7}\left(Y_{k}\right) \cong \mathbb{Z} \oplus A$, com A finito, então o homomorfismo $\left(\left(\tilde{f}_{k}\right)_{\#}\right)_{7}: \pi_{7}\left(Y_{k}\right) \rightarrow \pi_{7}\left(\mathbb{C} P^{3}\right)$ é tal que

$$
\left(\left(\tilde{f}_{k}\right)_{\#}\right)_{7}(n, a)= \begin{cases}2 k^{2} n, & \text { se } k \equiv 2,4,6(8), \\ k^{2} n, & \text { caso contrário }\end{cases}
$$


para todo $(n, a) \in \mathbb{Z} \oplus A$, onde $\pi_{7}\left(\mathbb{C} P^{3}\right) \cong \mathbb{Z}$.

Demonstração. Com efeito, das sequências de homotopia das fibrações $p_{k} \mathrm{e}$ $p$ e do diagrama geométrico 69.1, que relaciona estas fibrações, consideremos o diagrama algébrico abaixo.

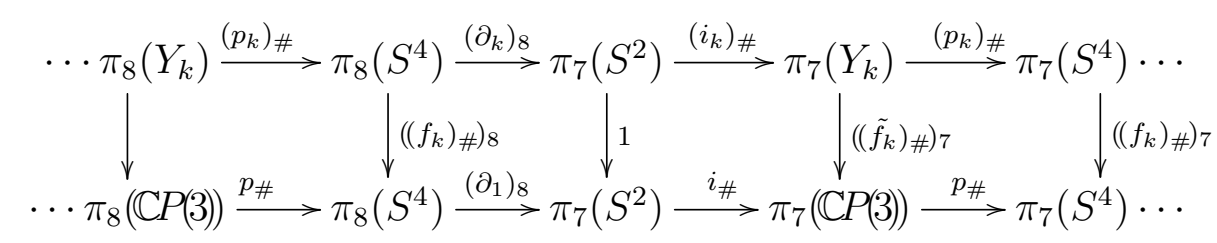

Primeiramente, vamos determinar $\left(\left(f_{k}\right)_{\#}\right)_{7}$ e $\left(\left(f_{k}\right)_{\#}\right)_{8}$. Denote por $\iota_{n} \in \pi_{n}\left(S^{n}\right)$ a classe de homotopia da identidade de $S^{n}$. Sabemos que $\left(f_{k}\right)_{\#}(\alpha)=\left(k \iota_{4}\right) \circ \alpha$. Por [Whg-78] (página 537), temos, para todo $k \in \mathbb{Z}, \alpha \in \pi_{n+1}\left(S^{4}\right), \beta \in$ $\pi_{4}\left(S^{4}\right)$, que $(k \beta) \circ \alpha=k(\beta \circ \alpha)+\left(\begin{array}{c}k \\ 2\end{array}\right)[\beta, \beta] \circ h_{0}(\alpha)-\left(\begin{array}{c}k+1 \\ 3\end{array}\right)[[\beta, \beta], \beta] \circ h_{1}(\alpha)$, onde $h_{0}, h_{1}$ são homomorfismos de Hopf-Hilton. Ainda, por [Whg-78] (página $535)$, se $n<12$, segue que $h_{1} \equiv 0$. Desta forma, como $6<12$ e $7<12$, então, $\left(\left(f_{k}\right)_{\#}\right)_{7}$ e $\left(\left(f_{k}\right)_{\#}\right)_{8}$ são tais que $\left(f_{k}\right)_{\#}(\alpha)=k \alpha+\left(\begin{array}{c}k \\ 2\end{array}\right)\left[\iota_{4}, \iota_{4}\right] h_{0}(\alpha)$.

Considere, agora, a seguinte sequência EHP

$$
\cdots \rightarrow \pi_{7}\left(S^{3}\right) \stackrel{E}{\rightarrow} \pi_{8}\left(S^{4}\right) \stackrel{H}{\rightarrow} \pi_{8}\left(S^{7}\right) \stackrel{P}{\rightarrow} \pi_{6}\left(S^{3}\right) \stackrel{E}{\rightarrow} \pi_{7}\left(S^{4}\right) \stackrel{H}{\rightarrow} \pi_{7}\left(S^{7}\right) \rightarrow \cdots
$$

Por [Tod-62] (página 43), $\pi_{7}\left(S^{4}\right)=<\nu_{4}>\oplus<E \nu^{\prime}>, \pi_{6}\left(S^{3}\right)=<\nu^{\prime}>, \pi_{8}\left(S^{7}\right)=<$ $\eta_{7}>, \pi_{8}\left(S^{4}\right)=<\nu_{4} \circ \eta_{7}>\oplus<E \nu^{\prime} \circ \eta_{7}>, \pi_{7}\left(S^{3}\right)=<\nu^{\prime} \circ \eta_{6}>$, onde $\nu_{4}$ é tal que $H\left(\nu_{4}\right)=\iota_{7}$, e $\pi_{7}\left(S^{4}\right) \ni\left[\iota_{4}, \iota_{4}\right]=2 \nu_{4}-E \nu^{\prime}$. Como $E: \nu^{\prime} \mapsto E \nu^{\prime}$, $E: \nu^{\prime} \circ \eta_{6} \mapsto E \nu^{\prime} \circ \eta_{7}$ e a sequência acima é exata, então os homomorfismos $E$ acima são injetores, o $P$ acima é o homomorfismo nulo e o $H$ da esquerda acima é sobrejetor, donde $H\left(\nu_{4} \circ \eta_{7}\right)=\eta_{7}$. Ainda, como a sequência é exata, vale destacar que $H\left(E \nu^{\prime} \circ \eta_{7}\right)=H\left(E\left(\nu^{\prime} \circ \eta_{6}\right)\right)=0$ e $H\left(E \nu^{\prime}\right)=0$.

Uniformizando a notação, notemos que $H=h_{0}$. Daí, $\left(\left(f_{k}\right)_{\#}\right)_{7}\left(E \nu^{\prime}\right)=k E \nu^{\prime}$ e $\left(\left(f_{k}\right)_{\#}\right)_{7}\left(\nu_{4}\right)=k \nu_{4}+\left(\begin{array}{c}k \\ 2\end{array}\right)\left(2 \nu_{4}-E \nu^{\prime}\right)=k^{2} \nu_{4}-\left(\begin{array}{c}k \\ 2\end{array}\right) E \nu^{\prime}$. Analogamente, 
$\left(\left(f_{k}\right)_{\#}\right)_{8}\left(E \nu^{\prime} \circ \eta_{7}\right)=k\left(E \nu^{\prime} \circ \eta_{7}\right)$ e $\left(\left(f_{k}\right)_{\#}\right)_{8}\left(\nu_{4} \circ \eta_{7}\right)=k^{2}\left(\nu_{4} \circ \eta_{7}\right)-\left(\begin{array}{l}k \\ 2\end{array}\right)\left(E \nu^{\prime} \circ\right.$ $\left.\eta_{7}\right)$. Analisemos, primeiramente, as possibilidades para $\left(\left(f_{k}\right)_{\#}\right)_{8}$. Desde que $\pi_{8}\left(S^{4}\right)=<\nu_{4} \circ \eta_{7}>\oplus<E \nu^{\prime} \circ \eta_{7}>\cong \mathbb{Z}_{2} \oplus \mathbb{Z}_{2}$, então os homomorfismos $\left(\left(f_{k}\right)_{\#}\right)_{8}$ são dados na tabela abaixo.

\begin{tabular}{|l|c|c|}
\hline & $\left(\left(f_{k}\right)_{\#}\right)_{8}\left(E \nu^{\prime} \circ \eta_{7}\right)$ & $\left(\left(f_{k}\right)_{\#}\right)_{8}\left(\nu_{4} \circ \eta_{7}\right)$ \\
\hline$k \equiv 0(4)$ & 0 & 0 \\
\hline$k \equiv 1(4)$ & $E \nu^{\prime} \circ \eta_{7}$ & $\nu_{4} \circ \eta_{7}$ \\
\hline$k \equiv 2(4)$ & 0 & $E \nu^{\prime} \circ \eta_{7}$ \\
\hline$k \equiv 3(4)$ & $E \nu^{\prime} \circ \eta_{7}$ & $\nu_{4} \circ \eta_{7}+E \nu^{\prime} \circ \eta_{7}$ \\
\hline
\end{tabular}

Em particular, se $k$ é ímpar, segue que $\left(\left(f_{k}\right)_{\#}\right)_{8}$ é sobrejetor. Consideremos o diagrama acima. Analisemos, agora, as possibilidades para $\left(\partial_{k}\right)_{8}$. Pela comutatividade do segundo quadrado, temos que $\left(\partial_{k}\right)_{8}=\left(\partial_{1}\right)_{8} \circ\left(\left(f_{k}\right)_{\#}\right)_{8}$. Algebricamente, como $\pi_{7}\left(S^{2}\right) \cong \mathbb{Z}_{2}$ e $\pi_{7}(\mathbb{C} P(3)) \cong \pi_{7}\left(S^{7}\right) \cong \mathbb{Z}$, segue que $i_{\#}: \mathbb{Z}_{2} \rightarrow \mathbb{Z}$ é o homomorfismo nulo. Daí, $\left(\partial_{1}\right)_{8}$ é sobrejetor e portanto, como $\pi_{8}\left(S^{4}\right) \cong \mathbb{Z}_{2} \oplus \mathbb{Z}_{2}$, segue que $\mathbb{Z}_{2} \cong \operatorname{ker}\left(\partial_{1}\right)_{8}=\operatorname{Im}\left(p_{\#}\right)$. Desde que $\pi_{8}(\mathbb{C} P(3)) \cong \pi_{8}\left(S^{7}\right) \cong \mathbb{Z}_{2}$, então $p_{\#}: \mathbb{Z}_{2} \rightarrow \mathbb{Z}_{2} \oplus \mathbb{Z}_{2}$ é injetor (desta forma, note que ambos $p_{\#}$ são injetores). Logo, se $k$ é ímpar, segue que $\left(\partial_{k}\right)_{8}$ é sobrejetor. Geometricamente, considerando $\pi_{8}(\mathbb{C} P(3))=<[q] \circ \eta_{7}>$, onde $q: S^{7} \rightarrow \mathbb{C} P(3)$ é a aplicação de Hopf sobre $\mathbb{C} P(3)$, então $p_{\#}\left([q] \circ \eta_{7}\right)=\nu_{4} \circ \eta_{7}$. Em particular, $\left(\partial_{1}\right)_{8}\left(\nu_{4} \circ \eta_{7}\right)=0$. Daí, como $\left(\partial_{1}\right)_{8}$ é sobrejetor e $\pi_{8}\left(S^{4}\right)=<$ $\nu_{4} \circ \eta_{7}>\oplus\left\langle E \nu^{\prime} \circ \eta_{7}>\right.$, então $\left(\partial_{1}\right)_{8}\left(E \nu^{\prime} \circ \eta_{7}\right) \neq 0$. Assim, pela tabela acima, se $k \equiv 2(4)$, então $\left(\partial_{1}\right)_{8}\left(\operatorname{Im}\left(\left(f_{k}\right)_{\#}\right)_{8}\right) \neq 0$ e obtemos novamente que $\left(\partial_{k}\right)_{8}=\left(\partial_{1}\right)_{8} \circ\left(\left(f_{k}\right)_{\#}\right)_{8}: \mathbb{Z}_{2} \oplus \mathbb{Z}_{2} \rightarrow \mathbb{Z}_{2}$ é sobrejetor. Portanto, do diagrama acima, se $k \not \equiv 0(4)$, então $\left(i_{k}\right)_{\#}: \pi_{7}\left(S^{2}\right) \rightarrow \pi_{7}\left(Y_{k}\right)$ é o homomorfismo nulo e $\left(p_{k}\right)_{\#}: \pi_{7}\left(Y_{k}\right) \rightarrow \pi_{7}\left(S^{4}\right)$ é injetor. Por fim, se $k \equiv 0(4)$, então, pela tabela acima, $\left(\left(f_{k}\right)_{\#}\right)_{8}$ é o homomorfismo nulo, donde, do diagrama acima, $\left(\partial_{k}\right)_{8}$ é o homomorfismo nulo e $\left(i_{k}\right)_{\#}: \pi_{7}\left(S^{2}\right) \rightarrow \pi_{7}\left(Y_{k}\right)$ é injetor. Analisemos, agora, 
as possibilidades para $\left(\left(f_{k}\right)_{\#}\right)_{7}$. Para isto, consideremos a continuação do diagrama algébrico acima.

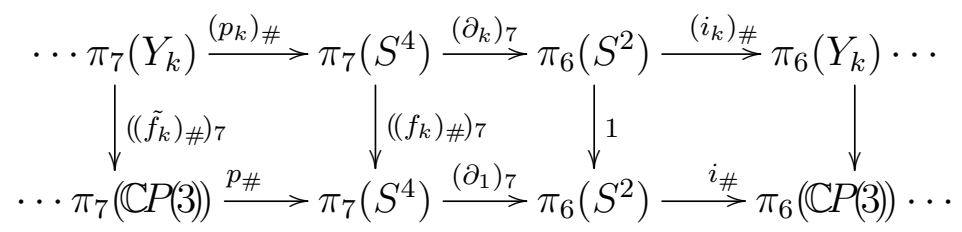

Desde que $\pi_{6}(\mathbb{C} P(3)) \cong \pi_{6}\left(S^{7}\right)=0$, então $i_{\#}$ é o homomorfismo nulo e $\left(\partial_{1}\right)_{7}$ é sobrejetor. Lembremos que $\pi_{7}(\mathbb{C} P(3))=<[q]>$, onde $q: S^{7} \rightarrow \mathbb{C} P^{3}$ é a aplicação de Hopf sobre $\mathbb{C} P(3)$. Como $p_{\#}([q])=\nu_{4}$ e $\pi_{7}\left(S^{4}\right)=\left\langle\nu_{4}\right\rangle \oplus<E \nu^{\prime}>$, então $\left(\partial_{1}\right)_{7}\left(\nu_{4}\right)=0$ e $\left(\partial_{1}\right)_{7}\left(E \nu^{\prime}\right)$ gera $\pi_{6}\left(S^{2}\right) \cong \mathbb{Z}_{12}$. Notemos que $\operatorname{Im}\left(\left(\tilde{f}_{k}\right)_{\#}\right)_{7} \subset<[q]>$ e portanto $\operatorname{Im}\left(\left(\left(f_{k}\right)_{\#}\right)_{7} \circ\left(p_{k}\right)_{\#}\right)=\operatorname{Im}\left(p_{\#} \circ\left(\left(\tilde{f}_{k}\right)_{\#}\right)_{7}\right) \subset<\nu_{4}>$. Daí, segue que $\left(\left(f_{k}\right)_{\#}\right)_{7}\left(\operatorname{ker}\left(\partial_{k}\right)_{7}\right)=\left(\left(f_{k}\right)_{\#}\right)_{7}\left(\operatorname{Im}\left(p_{k}\right)_{\#}\right) \subset<\nu_{4}>$ e portanto basta calcularmos $\left(\left(f_{k}\right)_{\#}\right)_{7}$ em $\operatorname{ker}\left(\partial_{k}\right)_{7}$, a fim de determinar $\left(\left(\tilde{f}_{k}\right)_{\#}\right)_{7}$. O lema auxiliar abaixo nos fornece uma caracterização de $\operatorname{ker}\left(\partial_{k}\right)_{7}$.

Lema 169. Temos que $\operatorname{ker}\left(\partial_{k}\right)_{7}=\operatorname{ker}\left(p r_{2} \circ\left(\left(f_{k}\right)_{\#}\right)_{7}\right)$, onde $\pi_{7}\left(S^{4}\right) \cong \mathbb{Z} \oplus \mathbb{Z}_{12}$ e pr $r_{2}: \mathbb{Z} \oplus \mathbb{Z}_{12} \rightarrow \mathbb{Z}_{12}$ é a projeção na segunda coordenada.

Demonstração. De fato, desde que $\left(\left(f_{k}\right)_{\#}\right)_{7}\left(\operatorname{ker}\left(\partial_{k}\right)_{7}\right) \subset<\nu_{4}>$, então $\left(p r_{2} \circ\right.$ $\left.\left(\left(f_{k}\right)_{\#}\right)_{7}\right)\left(\operatorname{ker}\left(\partial_{k}\right)_{7}\right) \subset 0$, donde $\operatorname{ker}\left(\partial_{k}\right)_{7} \subset \operatorname{ker}\left(p r_{2} \circ\left(\left(f_{k}\right)_{\#}\right)_{7}\right)$. Por outro lado, se $x \in \operatorname{ker}\left(p r_{2} \circ\left(\left(f_{k}\right)_{\#}\right)_{7}\right)$, então $\left(\left(f_{k}\right)_{\#}\right)_{7}(x) \in<\nu_{4}>$ e portanto $\left(\partial_{k}\right)_{7}(x)=$ $\left(\partial_{1}\right)_{7}\left(\left(\left(f_{k}\right)_{\#}\right)_{7}(x)\right)=0$, isto é, $x \in \operatorname{ker}\left(\partial_{k}\right)_{7}$. Assim, $\operatorname{ker}\left(\partial_{k}\right)_{7}=\operatorname{ker}\left(p r_{2} \circ\right.$ $\left.\left(\left(f_{k}\right)_{\#}\right)_{7}\right)$.

O próximo lema auxiliar, nos diz, em particular, que para dois valores de $k$ congruentes módulo 24 , os dois subgrupos, $\operatorname{ker}\left(p r_{2} \circ\left(\left(f_{k}\right)_{\#}\right)_{7}\right)$, de $\pi_{7}\left(S^{4}\right)$ correspondentes são iguais.

Lema 170. Temos que $\operatorname{pr}_{2} \circ\left(\left(f_{k+24}\right)_{\#}\right)_{7}=\operatorname{pr}_{2} \circ\left(\left(f_{k}\right)_{\#}\right)_{7}: \mathbb{Z} \oplus \mathbb{Z}_{12} \rightarrow \mathbb{Z}_{12}$.

Demonstração. Com efeito, lembremos que $\pi_{7}\left(S^{4}\right)=\left\langle\nu_{4}\right\rangle \oplus\left\langle E \nu^{\prime}\right\rangle$ e $\operatorname{Im}\left(p r_{2} \circ\left(\left(f_{k}\right)_{\#}\right)_{7}\right) \subset<E \nu^{\prime}>\cong \mathbb{Z}_{12}$. Assim, $p r_{2} \circ\left(\left(f_{k+24}\right)_{\#}\right)_{7}\left(E \nu^{\prime}\right)=(k+24) E \nu^{\prime}=$ 
$k E \nu^{\prime}=p r_{2} \circ\left(\left(f_{k}\right)_{\#}\right)_{7}\left(E \nu^{\prime}\right)$. Ainda, $p r_{2} \circ\left(\left(f_{k+24}\right)_{\#}\right)_{7}\left(\nu_{4}\right)=-\left(\begin{array}{c}k+24 \\ 2\end{array}\right) E \nu^{\prime}=$ $-\left(\begin{array}{l}k \\ 2\end{array}\right) E \nu^{\prime}=p r_{2} \circ\left(\left(f_{k}\right)_{\#}\right)_{7}\left(\nu_{4}\right)$. Logo, $p r_{2} \circ\left(\left(f_{k+24}\right)_{\#}\right)_{7}=p r_{2} \circ\left(\left(f_{k}\right)_{\#}\right)_{7}$.

Por este resultado, basta calcularmos $\operatorname{ker}\left(p r_{2} \circ\left(\left(f_{k}\right)_{\#}\right)_{7}\right)$, para $0 \leqslant k \leqslant 23$. Para $k=0$, tomamos $f_{0}=$ cte $: S^{4} \rightarrow S^{4}$ e obtemos $\operatorname{ker}\left(p r_{2} \circ\left(\left(f_{0}\right)_{\#}\right)_{7}\right)=$ $\operatorname{ker}(0) \cong \mathbb{Z} \oplus \mathbb{Z}_{12}$. Para $k=1$, tomamos $f_{1}=1_{S^{4}}: S^{4} \rightarrow S^{4}$ e obtemos $\operatorname{ker}\left(p r_{2} \circ\left(\left(f_{1}\right)_{\#}\right)_{7}\right)=\operatorname{ker}\left(p r_{2}\right) \cong \mathbb{Z}$. Seja $x \nu_{4}+y E \nu^{\prime} \in \operatorname{ker}\left(p r_{2} \circ\left(\left(f_{k}\right)_{\#}\right)_{7}\right)$, com $x, y \in \mathbb{Z}$. Desde que $\left(p r_{2} \circ\left(\left(f_{k}\right)_{\#}\right)_{7}\right)\left(x \nu_{4}+y E \nu^{\prime}\right)=\left(-\left(\begin{array}{c}k \\ 2\end{array}\right) x+k y\right) E \nu^{\prime}$, então $x$ e $y$ devem ser tais que $-\left(\begin{array}{c}k \\ 2\end{array}\right) x+k y \equiv 0(12)$. Para cada $k \geqslant 2$, por um lado, tomando o menor $x>0$ que satisfaz esta relação, obtemos um elemento $\alpha_{k}$ de ordem infinita e, por outro lado, tomando $x=0$, obtemos um elemento $\beta_{k}$ de ordem finita. Assim, escrevemos $\operatorname{ker}\left(p r_{2} \circ\left(\left(f_{k}\right)_{\#}\right)_{7}\right)=<\alpha_{k}, \beta_{k}>$ e obtemos a tabela abaixo.

Tabela 76.1:

\begin{tabular}{|l|c|c|c|}
\hline & $\left(\left(f_{k}\right)_{\#}\right)_{7}\left(E \nu^{\prime}\right)$ & $\left(\left(f_{k}\right)_{\#}\right)_{7}\left(\nu_{4}\right)$ & $\operatorname{ker}\left(p r_{2} \circ\left(\left(f_{k}\right)_{\#}\right)_{7}\right)$ \\
\hline$k=0$ & 0 & 0 & $<\nu_{4}, E \nu^{\prime}>\cong \mathbb{Z} \oplus \mathbb{Z}_{12}$ \\
\hline$k=1$ & $E \nu^{\prime}$ & $\nu_{4}$ & $<\nu_{4}>\cong \mathbb{Z}$ \\
\hline$k=2$ & $2 E \nu^{\prime}$ & $2^{2} \nu_{4}-E \nu^{\prime}$ & $<2 \nu_{4}+E \nu^{\prime}, 6 E \nu^{\prime}>\cong \mathbb{Z} \oplus \mathbb{Z}_{2}$ \\
\hline$k=3$ & $3 E \nu^{\prime}$ & $3^{2} \nu_{4}-3 E \nu^{\prime}$ & $<\nu_{4}+E \nu^{\prime}, 4 E \nu^{\prime}>\cong \mathbb{Z} \oplus \mathbb{Z}_{3}$ \\
\hline$k=4$ & $4 E \nu^{\prime}$ & $4^{2} \nu_{4}-6 E \nu^{\prime}$ & $<2 \nu_{4}, 3 E \nu^{\prime}>\cong \mathbb{Z} \oplus \mathbb{Z} 4$ \\
\hline$k=5$ & $5 E \nu^{\prime}$ & $5^{2} \nu_{4}-10 E \nu^{\prime}$ & $<\nu_{4}+2 E \nu^{\prime}>\cong \mathbb{Z}$ \\
\hline$k=6$ & $6 E \nu^{\prime}$ & $6^{2} \nu_{4}-3 E \nu^{\prime}$ & $<2 \nu_{4}+E \nu^{\prime}, 2 E \nu^{\prime}>\cong \mathbb{Z} \oplus \mathbb{Z}_{6}$ \\
\hline$k=7$ & $7 E \nu^{\prime}$ & $7^{2} \nu_{4}-9 E \nu^{\prime}$ & $<\nu_{4}+3 E \nu^{\prime}>\cong \mathbb{Z}$ \\
\hline$k=8$ & $8 E \nu^{\prime}$ & $8^{2} \nu_{4}-4 E \nu^{\prime}$ & $<\nu_{4}+2 E \nu^{\prime}, 3 E \nu^{\prime}>\cong \mathbb{Z} \oplus \mathbb{Z}_{4}$ \\
\hline$k=9$ & $9 E \nu^{\prime}$ & $9^{2} \nu_{4}$ & $<\nu_{4}, 4 E \nu^{\prime}>\cong \mathbb{Z} \oplus \mathbb{Z} 3$ \\
\hline$k=10$ & $10 E \nu^{\prime}$ & $10^{2} \nu_{4}-9 E \nu^{\prime}$ & $<2 \nu_{4}+3 E \nu^{\prime}, 6 E \nu^{\prime}>\cong \mathbb{Z} \oplus \mathbb{Z}_{2}$ \\
\hline
\end{tabular}




\begin{tabular}{|l|c|c|c|}
\hline$k=11$ & $11 E \nu^{\prime}$ & $11^{2} \nu_{4}-7 E \nu^{\prime}$ & $<\nu_{4}+5 E \nu^{\prime}>\cong \mathbb{Z}$ \\
\hline$k=12$ & 0 & $12^{2} \nu_{4}-6 E \nu^{\prime}$ & $<2 \nu_{4}, E \nu^{\prime}>\cong \mathbb{Z} \oplus \mathbb{Z}_{12}$ \\
\hline$k=13$ & $E \nu^{\prime}$ & $13^{2} \nu_{4}-6 E \nu^{\prime}$ & $<\nu_{4}+6 E \nu^{\prime}>\cong \mathbb{Z}$ \\
\hline$k=14$ & $2 E \nu^{\prime}$ & $14^{2} \nu_{4}-7 E \nu^{\prime}$ & $<2 \nu_{4}+E \nu^{\prime}, 6 E \nu^{\prime}>\cong \mathbb{Z} \oplus \mathbb{Z}_{2}$ \\
\hline$k=15$ & $3 E \nu^{\prime}$ & $15^{2} \nu_{4}-9 E \nu^{\prime}$ & $<\nu_{4}+3 E \nu^{\prime}, 4 E \nu^{\prime}>\cong \mathbb{Z} \oplus \mathbb{Z}_{3}$ \\
\hline$k=16$ & $4 E \nu^{\prime}$ & $16^{2} \nu_{4}$ & $<\nu_{4}, 3 E \nu^{\prime}>\cong \mathbb{Z} \oplus \mathbb{Z} 4$ \\
\hline$k=17$ & $5 E \nu^{\prime}$ & $17^{2} \nu_{4}-4 E \nu^{\prime}$ & $<\nu_{4}+8 E \nu^{\prime}>\cong \mathbb{Z}$ \\
\hline$k=18$ & $6 E \nu^{\prime}$ & $18^{2} \nu_{4}-9 E \nu^{\prime}$ & $<2 \nu_{4}+E \nu^{\prime}, 2 E \nu^{\prime}>\cong \mathbb{Z} \oplus \mathbb{Z}_{6}$ \\
\hline$k=19$ & $7 E \nu^{\prime}$ & $19^{2} \nu_{4}-3 E \nu^{\prime}$ & $<\nu_{4}+9 E \nu^{\prime}>\cong \mathbb{Z}$ \\
\hline$k=20$ & $8 E \nu^{\prime}$ & $20^{2} \nu_{4}-10 E \nu^{\prime}$ & $<2 \nu_{4}+E \nu^{\prime}, 3 E \nu^{\prime}>\cong \mathbb{Z} \oplus \mathbb{Z} 4$ \\
\hline$k=21$ & $9 E \nu^{\prime}$ & $21^{2} \nu_{4}-6 E \nu^{\prime}$ & $<\nu_{4}+2 E \nu^{\prime}, 4 E \nu^{\prime}>\cong \mathbb{Z} \oplus \mathbb{Z} 3$ \\
\hline$k=22$ & $10 E \nu^{\prime}$ & $22^{2} \nu_{4}-3 E \nu^{\prime}$ & $<2 \nu_{4}+3 E \nu^{\prime}, 6 E \nu^{\prime}>\cong \mathbb{Z} \oplus \mathbb{Z}{ }_{2}$ \\
\hline$k=23$ & $11 E \nu^{\prime}$ & $23^{2} \nu_{4}-E \nu^{\prime}$ & $<\nu_{4}+11 E \nu^{\prime}>\cong \mathbb{Z}$ \\
\hline$k=24$ & 0 & $24^{2} \nu_{4}$ & $<\nu_{4}, E \nu^{\prime}>\cong \mathbb{Z} \oplus \mathbb{Z} 12$ \\
\hline$k=25$ & $E \nu^{\prime}$ & $25^{2} \nu_{4}$ & $<\nu \nu_{4}>\mathbb{Z}$ \\
\hline
\end{tabular}

Consideremos o diagrama algébrico abaixo.

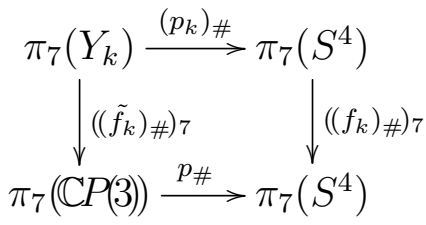

Finalmente, analisemos as possibilidades para $\left(\left(\tilde{f}_{k}\right)_{\#}\right)_{7}$. Observemos que $\operatorname{Im}\left(p_{k}\right)_{\#}=\operatorname{ker}\left(\partial_{k}\right)_{7}=\operatorname{ker}\left(p r_{2} \circ\left(\left(f_{k}\right)_{\#}\right)_{7}\right)$. Daí, se $\pi_{7}\left(Y_{k}\right) \cong \mathbb{Z} \oplus A$, com $A$ finito, então, sem perda de generalidade, podemos supor que

$$
\mathbb{Z} \oplus A \ni(1,0) \stackrel{\left(p_{k}\right)_{\#}}{\longmapsto} \alpha_{k} \in \operatorname{Im}\left(p_{k}\right)_{\#} .
$$


Ainda, usando a tabela acima, obtemos que

$$
\left(\left(f_{k}\right)_{\#}\right)_{7}\left(\alpha_{k}\right)= \begin{cases}2 k^{2} \nu_{4}, & \text { se } k \equiv 2,4,6(8), \\ k^{2} \nu_{4}, & \text { caso contrário. }\end{cases}
$$

Assim, desde que $p_{\#} \circ\left(\left(\tilde{f}_{k}\right)_{\#}\right)_{7}=\left(\left(f_{k}\right)_{\#}\right)_{7} \circ\left(p_{k}\right)_{\#}$ e $p_{\#}$ é um homomorfismo injetor tal que $p_{\#}([q])=\nu_{4}$, então

$$
\left(\left(\tilde{f}_{k}\right)_{\#}\right)_{7}(1,0)= \begin{cases}2 k^{2}[q], & \text { se } k \equiv 2,4,6(8), \\ k^{2}[q], & \text { caso contrário. }\end{cases}
$$

Logo, como $\left(\left(\tilde{f}_{k}\right)_{\#}\right)_{7}(n, a)=\left(\left(\tilde{f}_{k}\right)_{\#}\right)_{7}(n, 0)$, então, identificando $[q] \in \pi_{7}(\mathbb{C} P(3))$ com $1 \in \mathbb{Z}$, temos que a proposição 168 segue.

Agora, vamos determinar o homomorfismo $\omega^{\prime \prime}: \pi_{7}\left(Y_{k}\right) \rightarrow \pi_{2}\left(Y_{k}\right)$.

Teorema 171. Seja $k \in \mathbb{Z}$. Se $\pi_{7}\left(Y_{k}\right) \cong \mathbb{Z} \oplus A$, com A finito, então o homomorfismo $\omega^{\prime \prime}: \pi_{7}\left(Y_{k}\right) \rightarrow \pi_{2}\left(Y_{k}\right)$ é tal que

$$
\omega^{\prime \prime}(n, a)= \begin{cases} \pm 2|k| n, & \text { se } k \equiv 2,4,6(8), \\ \pm|k| n, & \text { caso contrário }\end{cases}
$$

para todo $(n, a) \in \mathbb{Z} \oplus A$, onde $\pi_{2}\left(Y_{k}\right) \cong \mathbb{Z}$.

Demonstração. De fato, pela proposição 167, temos o diagrama algébrico abaixo.

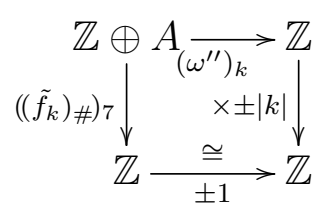

Daí, pela proposição 168, usando a descrição do homomorfismo $\left(\left(\tilde{f}_{k}\right)_{\#}\right)_{7}$, o resultado segue.

Vamos agora calcular $\pi_{7}\left(Y_{k}\right)$ por ser um problema interessante por si próprio. Faremos isto em três etapas, a saber, para $k \not \equiv 0(4)$, para $k \equiv 0(8)$ e para $k \equiv 4(8)$. 
Inicialmente, consideremos o diagrama algébrico abaixo, decorrente das sequências de homotopia das fibrações $p_{k}$ e $p$ e do diagrama geométrico 69.1, que relaciona estas fibrações.

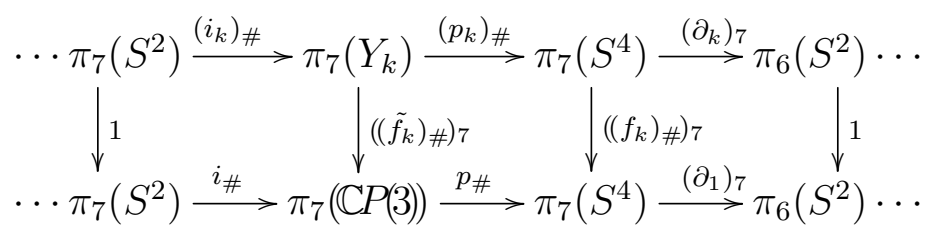

Proposição 172. Seja $k \not \equiv 0(4)$. Então $\pi_{7}\left(Y_{k}\right)$ é dado na tabela abaixo.

\begin{tabular}{|c|c|}
\hline & $\pi_{7}\left(Y_{k}\right)$ \\
\hline$k \equiv \pm 1, \pm 5(12)$ & $\mathbb{Z}$ \\
\hline$k \equiv \pm 2(12)$ & $\mathbb{Z} \oplus \mathbb{Z}_{2}$ \\
\hline$k \equiv \pm 3(12)$ & $\mathbb{Z} \oplus \mathbb{Z}_{3}$ \\
\hline$k \equiv 6(12)$ & $\mathbb{Z} \oplus \mathbb{Z}_{6}$ \\
\hline
\end{tabular}

Demonstração. De fato, se $k \not \equiv 0(4)$, segue, pela demonstração da proposição 168 , que $\left(p_{k}\right)_{\#}: \pi_{7}\left(Y_{k}\right) \rightarrow \pi_{7}\left(S^{4}\right)$ é injetor. Daí, pelo diagrama algébrico acima e pelo lema 169 , temos que $\pi_{7}\left(Y_{k}\right) \cong \operatorname{Im}\left(p_{k}\right)_{\#}=\operatorname{ker}\left(\partial_{k}\right)_{7}=$ $\operatorname{ker}\left(p r_{2} \circ\left(\left(f_{k}\right)_{\#}\right)_{7}\right)$. Como, pelo lema 170, $\operatorname{ker}\left(p r_{2} \circ\left(\left(f_{k+24}\right)_{\#}\right)_{7}\right)=\operatorname{ker}\left(p r_{2} \circ\right.$ $\left.\left(\left(f_{k}\right)_{\#}\right)_{7}\right)$, então, pela tabela 76.1 , que lista $\operatorname{ker}\left(p r_{2} \circ\left(\left(f_{k}\right)_{\#}\right)_{7}\right)$, na demonstração da proposição 168, o resultado segue.

Observação 173. Consideremos a tabela 76.1 na demonstração da proposição 168 e o lema 170. Notemos que, para dois valores de $k$ congruentes módulo 24, os dois subgrupos de $\pi_{7}\left(S^{4}\right)$ correspondentes, na tabela, são iguais, enquanto que, para dois valores de $k$ congruentes módulo 12 e não congruentes módulo 24, os dois subgrupos não são iguais, mas são isomorfos.

Seja agora $k \equiv 0(4)$. Pela demonstração da proposição 168, temos que $\left(i_{k}\right)_{\#}: \pi_{7}\left(S^{2}\right) \rightarrow \pi_{7}\left(Y_{k}\right)$ é injetor. Logo, da sequência de homotopia da fibração $p_{k}$, segue que 


$$
0 \rightarrow \pi_{7}\left(S^{2}\right) \stackrel{\left(i_{k}\right)_{\#}}{\rightarrow} \pi_{7}\left(Y_{k}\right) \stackrel{\left(p_{k}\right)_{\#}}{\rightarrow} \operatorname{ker}\left(p r_{2} \circ\left(\left(f_{k}\right)_{\#}\right)_{7}\right) \rightarrow 0
$$

é uma sequência exata curta. Analisemos primeiramente quando $k \equiv 0(8)$ e, em seguida, analisemos quando $k \equiv 4(8)$. Para isto, consideremos o lema algébrico abaixo.

Lema 174. Seja

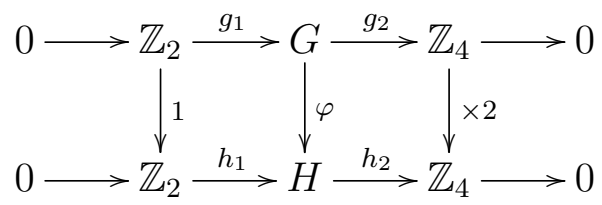

um diagrama algébrico comutativo tal que as linhas são exatas. Então $G \cong$ $\mathbb{Z}_{2} \oplus \mathbb{Z}_{4}$

Demonstração. De fato, como as linhas são exatas, então o grupo $G$ (e o grupo $H$ ) ou é isomorfo $\mathbb{Z}_{2} \oplus \mathbb{Z}_{4}$ ou é isomorfo a $\mathbb{Z}_{8}$. Suponhamos, por absurdo, que $G \cong \mathbb{Z}_{8}$. Por um lado, se $H \cong \mathbb{Z}_{2} \oplus \mathbb{Z}_{4}$, então $\varphi \circ g_{1}(1)=$ $\varphi(4)=4 \varphi(1)=0 \neq h_{1}(1)$, pois $h_{1}$ é injetor, o que não é possível. Por outro lado, se $H \cong \mathbb{Z}_{8}$, então $\varphi(4)=\varphi \circ g_{1}(1)=h_{1}(1)=4$. Ainda, como $g_{2}, h_{2}$ são sobrejetores, segue que $g_{2}(1), h_{2}(1) \in\{1,3\}$. Suponha, sem perda de generalidade, que $g_{2}(1)=h_{2}(1)=1$. Daí, $(\times 2) \circ g_{2}(1)=2$. Desde que $h_{2}(1)=1$, então $\varphi(1)$ não pode ser ímpar, e como $\varphi(4)=4$, segue que $\varphi(1)$ não pode ser par, o que é um absurdo. Logo, $G \cong \mathbb{Z}_{2} \oplus \mathbb{Z}_{4}$.

Observação 175. Consideremos o seguinte diagrama algébrico comutativo

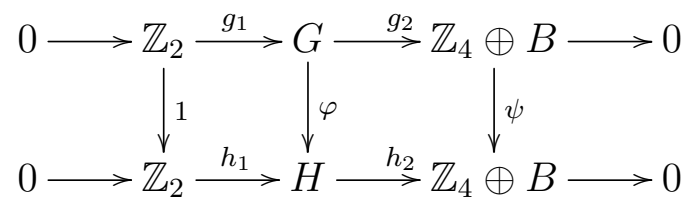

onde $B$ é igual a $\mathbb{Z}$ (ou $B$ é igual $a \mathbb{Z} \oplus \mathbb{Z}_{3}$ ). Notemos que, se $\psi$ restrito a $\mathbb{Z}_{4}$ é um homomorfismo de $\mathbb{Z}_{4}$ em $\mathbb{Z}_{4}$ dado pela multiplicação por 2 , então, pelo lema anterior, $G \cong \mathbb{Z}_{2} \oplus \mathbb{Z}_{4} \oplus \mathbb{Z}$ (ou $G \cong \mathbb{Z}_{2} \oplus \mathbb{Z}_{12} \oplus \mathbb{Z}$ ). 
Proposição 176. Seja $k \equiv 0(8)$. Então

$$
\pi_{7}\left(Y_{k}\right) \cong \begin{cases}\mathbb{Z} \oplus \mathbb{Z}_{2} \oplus \mathbb{Z}_{4}, & \text { se } k \equiv 8,16(24), \\ \mathbb{Z} \oplus \mathbb{Z}_{2} \oplus \mathbb{Z}_{12}, & \text { se } k \equiv 0(24) .\end{cases}
$$

Demonstração. De fato, seja $k=8$. Consideremos o diagrama algébrico abaixo, decorrente das sequências de homotopia das fibrações $p_{8}$ e $p_{4}$ e do diagrama geométrico dado pelo pullback $Y_{8}=f_{2}^{*}\left(Y_{4}\right)$.

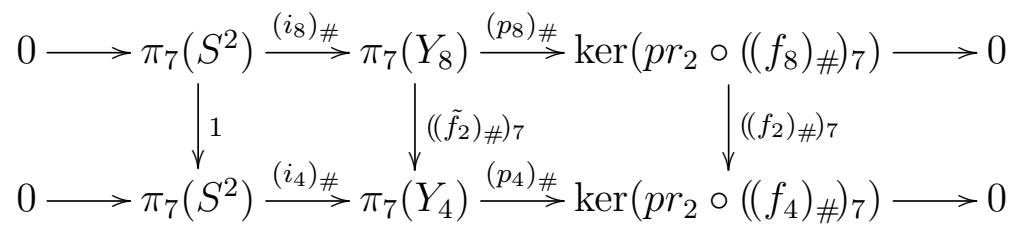

Pela tabela 76.1 na demonstração da proposição 168 , temos que $\operatorname{ker}\left(p r_{2} \circ\right.$ $\left.\left(\left(f_{8}\right)_{\#}\right)_{7}\right)=<\nu_{4}+2 E \nu^{\prime}, 3 E \nu^{\prime}>\cong \mathbb{Z} \oplus \mathbb{Z}_{4}, \operatorname{ker}\left(p r_{2} \circ\left(\left(f_{4}\right)_{\#}\right)_{7}\right)=<2 \nu_{4}, 3 E \nu^{\prime}>$ $\cong \mathbb{Z} \oplus \mathbb{Z}_{4}$ e $\left(\left(f_{2}\right)_{\#}\right)_{7}\left(3 E \nu^{\prime}\right)=6 E \nu^{\prime}$. Em particular, $\left(\left(f_{2}\right)_{\#}\right)_{7}$ restrito a $\mathbb{Z}_{4}$ é um homomorfismo de $\mathbb{Z}_{4}$ em $\mathbb{Z}_{4}$ dado pela multiplicação por 2. Daí, pelo lema 174 e pela observação $175, \pi_{7}\left(Y_{8}\right) \cong \mathbb{Z} \oplus \mathbb{Z}_{2} \oplus \mathbb{Z}_{4}$. Analogamente, considerando o diagrama algébrico dado pelo pullback $Y_{16}=f_{2}^{*}\left(Y_{8}\right)$, obtemos que $\pi_{7}\left(Y_{16}\right) \cong \mathbb{Z} \oplus \mathbb{Z}_{2} \oplus \mathbb{Z}_{4}$ e, considerando o diagrama algébrico dado pelo pullback $Y_{24}=f_{2}^{*}\left(Y_{12}\right)$, obtemos que $\pi_{7}\left(Y_{24}\right) \cong \mathbb{Z} \oplus \mathbb{Z}_{2} \oplus \mathbb{Z}_{12}$. Finalmente, desde que, pelo lema $170, \operatorname{ker}\left(p r_{2} \circ\left(\left(f_{k+24}\right)_{\#}\right)_{7}\right)=\operatorname{ker}\left(p r_{2} \circ\left(\left(f_{k}\right)_{\#}\right)_{7}\right)$, então o resultado segue.

Assim, resta calcular $\pi_{7}\left(Y_{k}\right)$ para $k \equiv 4(8)$. Ou melhor, desde que, pelo lema 170, $\operatorname{ker}\left(p r_{2} \circ\left(\left(f_{k+24}\right)_{\#}\right)_{7}\right)=\operatorname{ker}\left(p r_{2} \circ\left(\left(f_{k}\right)_{\#}\right)_{7}\right)$, resta determinar $\pi_{7}\left(Y_{4}\right), \pi_{7}\left(Y_{12}\right)$ e $\pi_{7}\left(Y_{20}\right)$.

Sejam $k, l \in \mathbb{Z}$, com $k=4 l$. Consideremos o diagrama algébrico abaixo, decorrente das sequências de homotopia das fibrações $p_{k}$ e $p_{4}$ e do diagrama geométrico dado pelo pullback $Y_{k}=f_{l}^{*}\left(Y_{4}\right)$. 


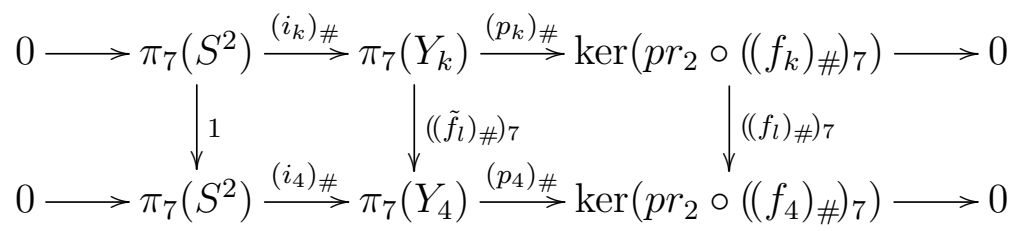

O próximo resultado nos diz que basta calcularmos $\pi_{7}\left(Y_{4}\right)$, a fim de determinar também $\pi_{7}\left(Y_{12}\right)$ e $\pi_{7}\left(Y_{20}\right)$.

Lema 177. Temos que ou $\pi_{7}\left(Y_{4}\right) \cong \pi_{7}\left(Y_{20}\right) \cong \mathbb{Z} \oplus \mathbb{Z}_{2} \oplus \mathbb{Z}_{4}$ e $\pi_{7}\left(Y_{12}\right) \cong$ $\mathbb{Z} \oplus \mathbb{Z}_{2} \oplus \mathbb{Z}_{12}$, ou $\pi_{7}\left(Y_{4}\right) \cong \pi_{7}\left(Y_{20}\right) \cong \mathbb{Z} \oplus \mathbb{Z}_{8}$ e $\pi_{7}\left(Y_{12}\right) \cong \mathbb{Z} \oplus \mathbb{Z}_{24}$.

Demonstração. De fato, consideremos o diagrama algébrico, como acima, dado pelo pullback $Y_{12}=f_{3}^{*}\left(Y_{4}\right)$.

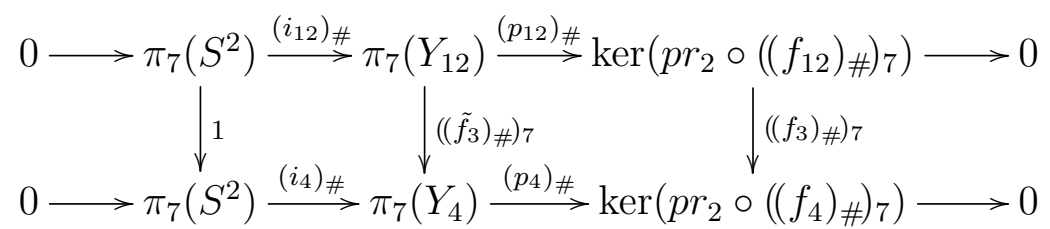

Pela tabela 76.1 na demonstração da proposição 168 , temos que $\operatorname{ker}\left(p r_{2} \circ\right.$ $\left.\left(\left(f_{12}\right)_{\#}\right)_{7}\right)=<2 \nu_{4}, E \nu^{\prime}>\cong \mathbb{Z} \oplus \mathbb{Z}_{12}, \operatorname{ker}\left(p r_{2} \circ\left(\left(f_{4}\right)_{\#}\right)_{7}\right)=<2 \nu_{4}, 3 E \nu^{\prime}>\cong \mathbb{Z} \oplus \mathbb{Z}_{4}$ e $\left(\left(f_{3}\right)_{\#}\right)_{7}\left(E \nu^{\prime}\right)=3 E \nu^{\prime}$. Em particular, $\left(\left(f_{2}\right)_{\#}\right)_{7}$ restrito a $\mathbb{Z}_{4}$ é um isomorfismo de $\mathbb{Z}_{4}$ em $\mathbb{Z}_{4}$. Logo, usando o lema dos cinco, se $\pi_{7}\left(Y_{4}\right) \cong \mathbb{Z} \oplus\left(\mathbb{Z}_{2} \oplus \mathbb{Z}_{4}\right)$, então $\pi_{7}\left(Y_{12}\right) \cong \mathbb{Z} \oplus \mathbb{Z}_{3} \oplus\left(\mathbb{Z}_{2} \oplus \mathbb{Z}_{4}\right)$ e, se $\pi_{7}\left(Y_{4}\right) \cong \mathbb{Z} \oplus \mathbb{Z}_{8}$, então $\pi_{7}\left(Y_{12}\right) \cong$ $\mathbb{Z} \oplus \mathbb{Z}_{3} \oplus \mathbb{Z}_{8}$. Analogamente, considerando o diagrama algébrico, como acima, dado pelo pullback $Y_{20}=f_{5}^{*}\left(Y_{4}\right)$, obtemos que se $\pi_{7}\left(Y_{4}\right) \cong \mathbb{Z} \oplus\left(\mathbb{Z}_{2} \oplus \mathbb{Z}_{4}\right)$, então $\pi_{7}\left(Y_{20}\right) \cong \mathbb{Z} \oplus\left(\mathbb{Z}_{2} \oplus \mathbb{Z}_{4}\right)$ e, se $\pi_{7}\left(Y_{4}\right) \cong \mathbb{Z} \oplus \mathbb{Z}_{8}$, então $\pi_{7}\left(Y_{20}\right) \cong \mathbb{Z} \oplus \mathbb{Z}_{8}$. 
Para o próximo resultado, consideremos o diagrama geométrico abaixo.

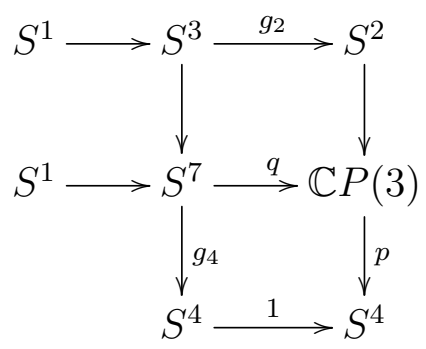

Seja $X:=f_{4}^{*}\left(S^{7}\right)$. Notemos que $\pi_{7}\left(Y_{4}\right) \cong \pi_{7}(X)$, pois $Y_{4}=\frac{X}{S^{1}}$. Consideremos o 4-esqueleto $X^{4}=S^{3} \cup_{\times 4} e^{4}$ de $X$. Notemos que $X^{4}$ é um espaço de Moore de tipo $\left(\mathbb{Z}_{4}, 3\right)$, isto é, $\tilde{H}_{3}\left(X^{4}, \mathbb{Z}\right) \cong \mathbb{Z}_{4}$ e $\tilde{H}_{i}\left(X^{4}, \mathbb{Z}\right)=0$, se $i \neq 4$. Por [MuShi-99], segue que $\pi_{6}\left(X^{4}\right) \cong \mathbb{Z}_{2} \oplus \mathbb{Z}_{2} \oplus \mathbb{Z}_{8}$. Assumindo que $\pi_{7}\left(X^{4}\right) \cong \mathbb{Z}_{2} \oplus \mathbb{Z}_{2} \oplus \mathbb{Z}_{4}$, então, da sequência de homotopia do par $\left(X, X^{4}\right)$, segue que $\pi_{7}(X)$ não pode ser isomorfo a $\mathbb{Z} \oplus \mathbb{Z}_{8}$. Daí, $\pi_{7}\left(Y_{4}\right) \cong \pi_{7}(X) \cong \mathbb{Z} \oplus \mathbb{Z}_{2} \oplus \mathbb{Z}_{4}$

Logo, pelas proposições 172 e 176, e via comunicação privada de Juno Mukai e, independentemente, de Jie $\mathrm{Wu}$, de que $\pi_{7}\left(X^{4}\right)$ é isomorfo a $\mathbb{Z}_{2} \oplus$ $\mathbb{Z}_{2} \oplus \mathbb{Z}_{4}$, segue a tabela abaixo.

\begin{tabular}{|c|c|}
\hline & $\pi_{7}\left(Y_{k}\right)$ \\
\hline$k \equiv \pm 1, \pm 5(12)$ & $\mathbb{Z}$ \\
\hline$k \equiv \pm 2(12)$ & $\mathbb{Z} \oplus \mathbb{Z}_{2}$ \\
\hline$k \equiv \pm 3(12)$ & $\mathbb{Z} \oplus \mathbb{Z}_{3}$ \\
\hline$k \equiv 6(12)$ & $\mathbb{Z} \oplus \mathbb{Z}_{6}$ \\
\hline$k \equiv \pm 4(12)$ & $\mathbb{Z} \oplus \mathbb{Z}_{2} \oplus \mathbb{Z}_{4}$ \\
\hline$k \equiv 0(12)$ & $\mathbb{Z} \oplus \mathbb{Z}_{2} \oplus \mathbb{Z}_{12}$ \\
\hline
\end{tabular}

\subsubsection{Variedades de Stiefel}

Seja $V_{k, k+l}$ a variedade de Stiefel de $k$-referenciais ortonormais em $\mathbb{R}^{k+l}$, com $k, l \geqslant 1$. Consideremos a seguinte fibração 


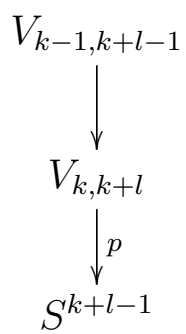

que projeta um $k$-referencial ortonormal em um 1-referencial ortonormal dado, por exemplo, por seu último vetor.

Desde que $S^{m}$ é $(m-1)$-conexo, para todo $m \geqslant 1$, então, pela sequência de homotopia da fibração acima, $V_{k, k+l}$ é $(l-1)$-conexo. Notemos ainda que $V_{k, k+l}$ é uma variedade de dimensão $n:=k(k+l)-\frac{1}{2} k(k+1)$. Desta forma, estamos interessados em $\omega^{\prime \prime}: \pi_{n+1}\left(V_{k, k+l}\right) \rightarrow \pi_{2}\left(V_{k, k+l}\right)$.

Proposição 178. Seja $V_{k, k+l}$ a variedade de Stiefel de $k$-referenciais ortonormais em $\mathbb{R}^{k+l}$, com $k, l \geqslant 1$. Então $\omega^{\prime \prime} \equiv 0$, exceto para $V_{1,3} \approx S^{2}$ quando $\omega^{\prime \prime}=\times( \pm 1)$.

Demonstração. De fato, para $V_{1,3} \approx S^{2}$, temos, pela proposição 151 , que $\omega^{\prime \prime}=\times( \pm 1)$. Vamos verificar, para os demais casos, que $\omega^{\prime \prime}$ é o homomorfismo nulo. Se $l \geqslant 3$, então $V_{k, k+l}$ é, pelo menos, 2-conexo, donde $\pi_{2}\left(V_{k, k+l}\right)=0$ e portanto $\omega^{\prime \prime} \equiv 0$. Seja $l=1$. Então $V_{k, k+1}=S O(k+1)$ e, a partir da fibração acima, obtemos a seguinte fibração:

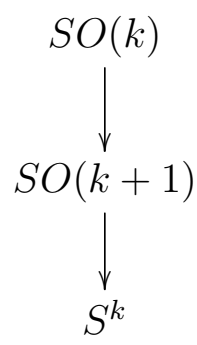

Se $k=1$, então $\pi_{2}\left(V_{1,2}\right) \cong \pi_{2}(S O(2)) \cong \pi_{2}\left(S^{1}\right)=0$ e o resultado segue. Se $k=2$, temos que $\pi_{2}\left(V_{2,3}\right) \cong \pi_{2}(S O(3)) \cong \pi_{2}(\mathbb{R} P(3)) \cong \pi_{2}\left(S^{3}\right)=0$ e o resultado também segue. Se $k \geqslant 3$, então, usando a sequência de homotopia 
da fibração $p$, obtemos que $\pi_{2}(S O(k+1))=0=\pi_{2}(S O(k))$. Assim, se $l=1$, concluímos que $\omega^{\prime \prime} \equiv 0$. Finalmente, seja $l=2$. Se $k=2$, então, como $\pi_{2}(S O(3))=0$, temos que o $S^{2}$-fibrado sobre $S^{3}, V_{2,4}$, é o fibrado trivial $S^{3} \times S^{2}$. Daí, pelo lema 148, obtemos que $\omega^{\prime \prime}$ é o homomorfismo nulo. Se $k \geqslant 3$, então, da sequência de homotopia da fibração $p, \pi_{2}\left(V_{k, k+2}\right) \cong$ $\pi_{2}\left(V_{k-1, k+1}\right) \cong \pi_{2}\left(V_{2,4}\right) \cong \mathbb{Z}$. Ainda, desde que $k(k+2)-\frac{1}{2} k(k+1)+1=$ $\frac{(k+2)(k+1)}{2}=\left(\begin{array}{c}k+2 \\ 2\end{array}\right)$, então queremos calcular $\pi_{\left(\begin{array}{c}k+2 \\ 2\end{array}\right)}\left(V_{k, k+2}\right)$. Observemos que $\left(\begin{array}{c}k+2 \\ 2\end{array}\right)>2(k+1)-1$ e $\left(\begin{array}{c}k+2 \\ 2\end{array}\right)>k+1$, com $k \geqslant 3$. Sendo $l=2$, notemos que a base da fibração $p$ é $S^{k+1}$, a base da fibração construída da mesma forma para a fibra de $p$ é $S^{k}$, a base da fibração construída do mesmo modo para a fibra da fibração anterior é $S^{k-1}$ e assim por diante. Desta forma, como a esfera de maior dimensão que aparece nesta análise é $S^{k+1}$, segue, das desigualdades anteriores, por [Se-51], que $\pi_{\left(\begin{array}{c}k+2 \\ 2\end{array}\right)}\left(V_{k, k+2}\right)$ é finito, para $k \geqslant 3$. Logo, $\omega^{\prime \prime}: \pi_{\left(\begin{array}{c}k+2 \\ 2\end{array}\right)}\left(V_{k, k+2}\right) \rightarrow \pi_{2}\left(V_{k, k+2}\right)$ é o homomorfismo nulo, para $k \geqslant 3$. Desta forma, o resultado segue.

\subsection{Cálculos parciais de $\omega^{\prime \prime}$}

Nesta seção, sejam $n \geqslant 2$ e $Y$ uma $n$-variedade fechada, conexa. Já analisamos $\omega^{\prime \prime}: \pi_{n+1}(Y) \rightarrow \pi_{2}(Y)$ para vários espaços $Y$, por exemplo, para superfícies, 3-variedades, $S^{a}$-fibrados sobre $S^{b}$ e variedades de Stiefel (reais). Analisaremos, agora, o homomorfismo $\omega^{\prime \prime}$, para algumas 4-variedades, alguns $F^{a}$-fibrados sobre $B^{b}$ e algumas variedades de Grassmann (reais), sendo $F, B$ variedades fechadas, conexas. 


\subsubsection{4-variedades}

Primeiramente, vamos fazer algumas considerações sobre os grupos de homologia de uma 4-variedade fechada, simplesmente conexa. Seja $Y$ uma 4-variedade fechada, simplesmente conexa. Em particular, notemos que $Y$ é orientável, pois todo laço em $Y$ é homotópico ao laço trivial. Consideremos os grupos de homologia de $Y$ com coeficientes em $\mathbb{Z}$. Temos, pelo teorema de Hurewicz, que $\pi_{1}(Y)=0=H_{1}(Y ; \mathbb{Z})$ e $\pi_{2}(Y) \cong H_{2}(Y ; \mathbb{Z})$. Ainda, pela dualidade de Poincaré (vide [V-94], página 165), sendo $Y$ orientável, $H_{2}(Y ; \mathbb{Z}) \cong$ $H^{2}(Y ; \mathbb{Z}), H_{3}(Y ; \mathbb{Z}) \cong H^{1}(Y ; \mathbb{Z})$ e $H_{4}(Y ; \mathbb{Z}) \cong \mathbb{Z}$. Daí, pelo teorema dos coeficientes universais para cohomologia (vide [V-94], página 84), temos que $H_{2}(Y ; \mathbb{Z}) \cong H^{2}(Y ; \mathbb{Z}) \cong \operatorname{Hom}\left(H_{2}(Y ; \mathbb{Z}), \mathbb{Z}\right)$ e $H_{3}(Y ; \mathbb{Z}) \cong H^{1}(Y ; \mathbb{Z}) \cong$ $\operatorname{Hom}\left(H_{1}(Y ; \mathbb{Z}), \mathbb{Z}\right)$, pois, respectivamente, $\operatorname{Ext}\left(H_{1}(Y ; \mathbb{Z}), \mathbb{Z}\right)=\operatorname{Ext}(0, \mathbb{Z})=0$ e $\operatorname{Ext}\left(H_{0}(Y ; \mathbb{Z}), \mathbb{Z}\right)=\operatorname{Ext}(\mathbb{Z}, \mathbb{Z})=0$. Desde que $H_{1}(Y ; \mathbb{Z})=0$, então $H_{3}(Y ; \mathbb{Z})=0$. Ainda, como $H_{2}(Y ; \mathbb{Z}) \cong \operatorname{Hom}\left(H_{2}(Y ; \mathbb{Z}), \mathbb{Z}\right)$ e $H_{2}(Y ; \mathbb{Z})$ é um grupo abeliano finitamente gerado, então $H_{2}(Y ; \mathbb{Z})$ é livre de torção, isto é, $H_{2}(Y ; \mathbb{Z}) \cong \mathbb{Z}^{k}$, para algum $k \in \mathbb{Z}, k \geqslant 0$. Desta forma, ao determinarmos o segundo número de Betti de $Y$, conhecemos todos os grupos de homologia de $Y$.

Agora, seja $Y$ uma 4-variedade fechada, não necessariamente simplesmente conexa. Seja $\tilde{Y}$ o revestimento universal de $Y$ e seja $k$ o segundo número de Betti de $\widetilde{Y}$. Suponha que $\widetilde{Y}$ é compacto. Daí, $\widetilde{Y}$ é uma 4variedade fechada, simplesmente conexa. Notemos que $\pi_{i}(Y) \cong \pi_{i}(\widetilde{Y})$, para todo $i \geqslant 2$. Em particular, $\pi_{2}(Y) \cong \pi_{2}(\widetilde{Y}) \cong H_{2}(\widetilde{Y} ; \mathbb{Z}) \cong \mathbb{Z}^{k}$ e estamos interessados em estudar $\omega^{\prime \prime}: \pi_{5}(Y) \rightarrow \pi_{2}(Y) \cong \mathbb{Z}^{k}$.

O próximo resultado determina $\omega^{\prime \prime}$ completamente, quando $0 \leqslant k \leqslant 2$.

Proposição 179. Seja $Y$ uma 4-variedade. Sejam $\widetilde{Y}$ o revestimento universal de $Y$ e $k$ o segundo número de Betti de $\tilde{Y}$. Se $0 \leqslant k \leqslant 2$, então $\omega^{\prime \prime} \equiv 0$, 
exceto para $\tilde{Y}=\mathbb{C} P(2)$ quando $\omega^{\prime \prime}=\times( \pm 1)$.

Demonstração. Com efeito, se $Y$ é aberta, então, pelo lema $148, \omega^{\prime \prime} \equiv 0$. Seja $Y$ fechada. Notemos que $\widetilde{Y}$ é uma 4-variedade sem bordo, simplesmente conexa. Se $\widetilde{Y}$ é aberta, então, pelo lema 148 , $\omega^{\prime \prime} \equiv 0$. Seja $\widetilde{Y}$ fechada. Daí, por [BaBa-13], se $0 \leqslant k \leqslant 2$, então $\tilde{Y} \in\left\{S^{4}, \mathbb{C} P(2), S^{2} \times\right.$ $\left.S^{2}, \mathbb{C} P(2) \# \mathbb{C} P(2), \mathbb{C} P(2) \# \overline{\mathbb{C} P}(2)\right\}$, onde $\overline{\mathbb{C} P}(2)$ representa $\mathbb{C} P(2)$ com orientação contrária. Se $\widetilde{Y}=S^{4}$, então $\pi_{2}(Y) \cong \pi_{2}\left(S^{4}\right)=0$ e portanto $\omega^{\prime \prime}$ é o homomorfismo nulo. Se $\widetilde{Y}=S^{2} \times S^{2}$, então $\pi_{5}(Y) \cong \mathbb{Z}_{2} \oplus \mathbb{Z}_{2}$ e $\pi_{2}(Y) \cong \mathbb{Z} \oplus \mathbb{Z}$, donde $\omega^{\prime \prime} \equiv 0$. Agora, por [BaBa-13], notemos que existem $S^{1}$-fibrações sobre $\mathbb{C} P(2) \# \mathbb{C} P(2)$ e sobre $\mathbb{C} P(2) \# \overline{\mathbb{C} P}(2)$, tendo como espaço total $S^{2} \times S^{3}$. Assim, se $\widetilde{Y}$ é ou $\mathbb{C} P(2) \# \mathbb{C} P(2)$, ou $\mathbb{C} P(2) \# \overline{\mathbb{C} P}(2)$, então $\omega^{\prime \prime}$ é um homomorfismo de $\pi_{5}(Y) \cong \pi_{5}(\tilde{Y}) \cong \pi_{5}\left(S^{2} \times S^{3}\right) \cong \mathbb{Z}_{2} \oplus \mathbb{Z}_{2}$ em $\pi_{2}(Y) \cong \pi_{2}(\tilde{Y}) \cong$ $\pi_{2}\left(S^{2} \times S^{3}\right) \oplus \pi_{1}\left(S^{1}\right) \cong \mathbb{Z} \oplus \mathbb{Z}$ e portanto $\omega^{\prime \prime}$ é novamente o homomorfismo nulo. Seja $\tilde{Y}=\mathbb{C} P(2)$. Como $\omega_{Y}^{\prime \prime}([f])=\mu\left(\tilde{\omega}\left(\tilde{y}_{0}, \tilde{f}\right)\right)=\omega_{\mathbb{C} P(2)}^{\prime \prime}([\tilde{f}])$, onde $\tilde{f}$ é o levantamento de $f$ tal que $\tilde{f}\left(x_{0}\right)=\tilde{y}_{0}$ e, pela proposição $164, \omega_{\mathbb{C} P(2)}^{\prime \prime}$ é um isomorfismo de $\mathbb{Z}$ em $\mathbb{Z}$, então $\omega_{Y}^{\prime \prime}$ também o é, isto é, $\omega_{Y}^{\prime \prime}=\times( \pm 1)$.

Se $k \geqslant 3$, então, por $[\mathrm{BaBa}-13], \pi_{5}^{\mathbb{Q}}(\tilde{Y}) \cong \mathbb{Q}^{\frac{\left(k^{2}-1\right)\left(k^{2}-4\right)}{4}}$, onde $\pi_{5}^{\mathbb{Q}}(Y):=$ $\pi_{5}(Y) \otimes \mathbb{Q}$. Em particular, segue que $\pi_{5}(Y)$ não é finito. Desta forma, para $k \geqslant 3$, ainda não conhecemos $\omega^{\prime \prime}: \pi_{5}(Y) \rightarrow \pi_{2}(Y)$.

Observação 180. Considerações sobre racionalmente elíptico e racionalmente hiperbólico:

Seja $Y$ uma 4-variedade fechada, simplesmente conexa. Dizemos que o espaço $Y$ é racionalmente elíptico se $\sum_{i \geqslant 2} \operatorname{dim} \pi_{i}^{\mathbb{Q}}(Y)<\infty$, e dizemos que $Y$ é racionalmente hiperbólico, caso contrário, onde $\pi_{i}^{\mathbb{Q}}(Y):=\pi_{i}(Y) \otimes \mathbb{Q}$ é um espaço vetorial sobre $\mathbb{Q}$. Por $[\mathrm{FeOTa-08]}$ (página 109), $Y$ é racionalmente elíptico se, e somente se, $Y \in\left\{S^{4}, \mathbb{C} P(2), S^{2} \times S^{2}, \mathbb{C} P(2) \# \mathbb{C} P(2), \mathbb{C} P(2) \# \overline{\mathbb{C} P}(2)\right\}$, 
o que ocorre, por [BaBa-13], se, e só se, o segundo número de Betti de Y é menor ou igual a 2 .

\subsection{2 $\quad F^{a}$-fibrados sobre $B^{b}$}

Sejam $a, b \in \mathbb{Z}, a, b \geqslant 1$. Seja $p: Y \rightarrow B^{b}$ um $F^{a}$-fibrado sobre $B^{b}$, com $F, B$ variedades fechadas, conexas. Assim, $n=a+b$ e $Y$ é uma variedade $(a+b)$-dimensional. Desta forma, estamos interessados em estudar $\omega^{\prime \prime}$ : $\pi_{a+b+1}(Y) \rightarrow \pi_{2}(Y)$. Nesta subseção, mais especificamente, vamos analisar $\omega^{\prime \prime}$ quando a fibra ou a base é revestida por uma esfera, ou quando a fibra ou a base é uma variedade de dimensão menor ou igual a 3.

Primeiramente, vejamos alguns resultados auxiliares.

Lema 181. Se $F=S^{a}$ e $a \geqslant b$, então $\omega^{\prime \prime} \equiv 0$.

Demonstração. Com efeito, se $a \geqslant b$, então $S^{a}$ é $(b-1)$-conexa e portanto, por [Hu-94] (página 21), $p$ admite uma seção. Logo, pelo lema 148, $\omega^{\prime \prime} \equiv 0$

Proposição 182. Seja $F=\mathbb{R} P(a)$, com $a=2 a^{\prime}$, para algum $a^{\prime} \geqslant 1$. Se $a \geqslant b$, então p admite uma seção. Neste caso, $\omega^{\prime \prime} \equiv 0$.

Demonstração. De fato, sendo $a=2 a^{\prime}$, temos que $\mathbb{R} P(a)$ é não orientável. Logo, $Y$ é não orientável, pois $U \times \mathbb{R} P(a) \approx p^{-1}(U)$ é uma subvariedade aberta não orientável de $Y$, para algum $U$ aberto em $B$. Seja $q: Y^{\prime} \rightarrow Y$ o revestimento duplo orientável de $Y$. Daí, $p \circ q: Y^{\prime} \rightarrow B$ é uma fibração que tem como fibra típica o revestimento duplo orientável de $\mathbb{R} P(a)$, isto é, $S^{a}$, conforme o diagrama geométrico abaixo. 


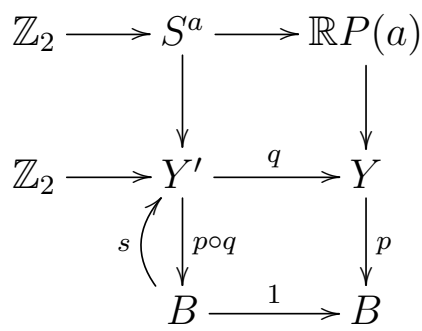

Se $a \geqslant b$, então $S^{a}$ é $(b-1)$-conexa e portanto, por [Hu-94] (página 21), $p \circ q$ admite uma seção $s$. Logo, $q \circ s$ é uma seção de $p$ e o resultado principal segue. Daí, pelo lema 148, $\omega^{\prime \prime} \equiv 0$.

Lema 183. Se $S^{a}$ reveste $F, S^{b}$ reveste $B$ e $a<b, \operatorname{com}(a, b) \neq(2,4)$, então $\omega^{\prime \prime} \equiv 0$.

Demonstração. De fato, primeiramente, notemos que $\pi_{i}(F) \cong \pi_{i}\left(S^{a}\right)$ e $\pi_{i}(B) \cong \pi_{i}\left(S^{b}\right)$, para todo $i \geqslant 2$. Daí, se $(a, b) \notin\{(2,3),(2,4)\}$, então, pela sequência de homotopia da fibração $p$, de modo análogo ao que foi feito para $S^{a}$-fibrados sobre $S^{b}$ na proposição 166 , temos que o resultado segue. Seja $Y$ um $F^{2}$-fibrado sobre $B^{3}$. Pelo teorema de classificação de superfícies fechadas, notemos que $F \in\left\{S^{2}, \mathbb{R} P(2)\right\}$. Consideremos o pullback, $Y^{\prime}$, de $Y$ pelo revestimento universal de $B$.

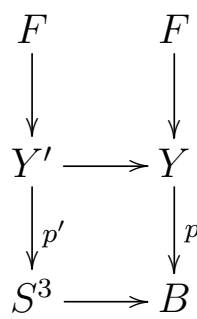

Usando as sequências de homotopia das fibrações $p^{\prime}$ e $p$, consideremos o diagrama algébrico abaixo induzido pelo diagrama geométrico acima.

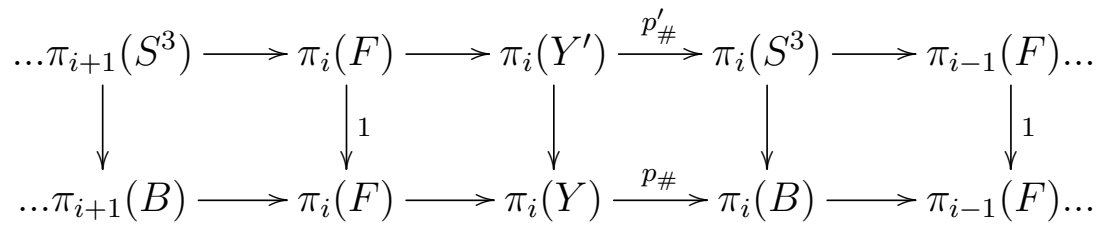


Pelo lema dos cinco, segue que $\pi_{i}\left(Y^{\prime}\right) \cong \pi_{i}(Y)$, para todo $i \geqslant 2$. Primeiramente, seja $F=S^{2}$. Como, por [Sm-59], $\operatorname{Diff}\left(S^{2}\right) \sim O(3)$, então $\pi_{2}\left(\operatorname{Diff}\left(S^{2}\right)\right) \cong \pi_{2}(O(3))=\pi_{2}(S O(3)) \cong \pi_{2}(\mathbb{R} P(3)) \cong \pi_{2}\left(S^{3}\right)=0$. Daí, $Y^{\prime} \approx S^{3} \times S^{2}, \pi_{6}(Y) \cong \pi_{6}\left(Y^{\prime}\right) \cong \mathbb{Z}_{12} \oplus \mathbb{Z}_{12}$ e $\pi_{2}(Y) \cong \pi_{2}\left(Y^{\prime}\right) \cong \mathbb{Z}$. Logo, $\omega^{\prime \prime}: \pi_{6}(Y) \rightarrow \pi_{2}(Y)$ é o homomorfismo nulo. Seja agora $F=\mathbb{R} P(2)$. Por [EE-69], temos que $\operatorname{Diff}^{+}(\mathbb{R} P(2)) \sim S O(3)$. Daí, analogamente, obtemos que $\omega^{\prime \prime}: \mathbb{Z}_{12} \oplus \mathbb{Z}_{12} \rightarrow \mathbb{Z}$ é o homomorfismo nulo.

Lema 184. Se $\pi_{1}(B)$ é infinito, então $\omega^{\prime \prime} \equiv 0$.

Demonstração. De fato, consideremos a sequência de homotopia da fibração $p, \cdots \pi_{2}(B) \rightarrow \pi_{1}(F) \rightarrow \pi_{1}(Y) \stackrel{p_{\#}}{\rightarrow} \pi_{1}(B) \rightarrow 1$. Desde que $\pi_{1}(B)$ é infinito, então $\pi_{1}(Y)$ também o é. Logo, pelo lema $148, \omega^{\prime \prime} \equiv 0$.

Em particular, se $B$ ou é $S^{1}$, ou é uma superfície $K(\pi, 1)$, ou é uma 3variedade $K(\pi, 1)$, ou é revestida por $\mathbb{R} \times S^{2}$, então, pelo lema anterior, temos que $\omega^{\prime \prime} \equiv 0$.

Os próximos dois resultados generalizam a proposição 166, que trata de $S^{a}$-fibrados sobre $S^{b}$.

Proposição 185. Se $F=S^{a}, S^{b}$ reveste $B e(a, b) \neq(2,4)$, então $\omega^{\prime \prime} \equiv 0$.

Demonstração. Com efeito, se $a \geqslant b$, então o resultado segue pelo lema 181. Se $a<b$, então o resultado segue pelo lema 183 .

Proposição 186. Se $F=\mathbb{R} P\left(2 a^{\prime}\right)$, $S^{b}$ reveste $B$ e $\left(2 a^{\prime}, b\right) \neq(2,4)$, então $\omega^{\prime \prime} \equiv 0$.

Demonstração. Com efeito, se $2 a^{\prime} \geqslant b$, então o resultado segue pela proposição 182. Se $2 a^{\prime}<b$, então o resultado segue pelo lema 183.

Usando o teorema de classificação de superfícies fechadas e a classificação de 3-variedades fechadas, obtemos o resultado abaixo. 
Proposição 187. Se ou $F=S^{a}$, ou $F=\mathbb{R} P\left(2 a^{\prime}\right)$, e $b \leqslant 3$, então $\omega^{\prime \prime} \equiv 0$.

Demonstração. De fato, se $B$ ou é $S^{1}$, ou é uma superfície $K(\pi, 1)$, ou é uma 3-variedade $K(\pi, 1)$, ou é revestida por $\mathbb{R} \times S^{2}$, então $\pi_{1}(B)$ é infinito e portanto, pelo lema 184 , segue que $\omega^{\prime \prime} \equiv 0$. Pela classificação de superfícies e 3-variedades fechadas, resta analisar quando $B$ é revestida por $S^{b}$, com $b=2,3$. Neste caso, pelas proposições 185 e 186 , temos que o resultado segue.

O próximo resultado trata de fibrados, em que tanto a fibra, quanto a base, tem dimensão menor ou igual a 3.

Proposição 188. Sejam $a, b \leqslant 3$. Se $F$ não é revestida por $\mathbb{R} \times S^{2}$, ou $B$ não é revestida por $S^{2}$, então $\omega^{\prime \prime} \equiv 0$.

Demonstração. Com efeito, pelo teorema de classificação de superfícies fechadas e pela classificação de 3 -variedades fechadas, temos que $B, F \in$ $\left\{S^{1}, S^{2}, \mathbb{R} P(2), K\left(\pi_{2}, 1\right), \frac{S^{3}}{G}, K\left(\pi_{3}, 1\right), M^{3}\right\}$, onde $K\left(\pi_{j}, 1\right)$ denota uma $j$-variedade $K(\pi, 1)$, com $j=1,2$, e $M$ denota uma 3 -variedade revestida por $\mathbb{R} \times S^{2}$ (ou seja, por [Tol-74], $M \in\left\{S^{1} \times S^{2}, \mathbb{R} P(3) \# \mathbb{R} P(3), S^{1} \tilde{\times} S^{2}, S^{1} \times\right.$ $\mathbb{R} P(2)\})$. Se $\pi_{1}(B)$ é infinito, segue, pelo lema 184 , que $\omega^{\prime \prime} \equiv 0$. Seja $\pi_{1}(B)$ finito. Logo, $B \in\left\{S^{2}, \mathbb{R} P(2), \frac{S^{3}}{G}\right\}$. Se $F$ é $K(\pi, 1)$, então, da sequência de homotopia da fibração $p$, obtemos que $\pi_{2}(Y) \leqslant \pi_{2}(B) \leqslant \mathbb{Z}$, e $\pi_{a+b+1}(Y) \cong$ $\pi_{a+b+1}(B) \cong \pi_{a+b+1}\left(S^{3}\right) \cong \pi_{a+b+1}\left(S^{2}\right)$ finito (pois $a+b+1 \geqslant 4$ ) e portanto $\omega^{\prime \prime}$ é o homomorfismo nulo. Seja $F \in\left\{S^{2}, \mathbb{R} P(2), \frac{S^{3}}{G}, M\right\}$. Inicialmente, seja $B=\frac{S^{3}}{G}$. Se $F=M$, então $\pi_{1}(Y)$ é infinito (pois $\pi_{1}(M) \leqslant \pi_{1}(Y)$ e $\pi_{1}(M)$ é infinito) e portanto, pelo lema $148, \omega^{\prime \prime} \equiv 0$. Se $F=\frac{S^{3}}{G}$, então $\pi_{2}(Y)=0$ e portanto $\omega^{\prime \prime} \equiv 0$. Se $F \in\left\{S^{2}, \mathbb{R} P(2)\right\}$, segue, pelo lema 183, que $\omega^{\prime \prime} \equiv 0$. Finalmente, seja $B \in\left\{S^{2}, \mathbb{R} P(2)\right\}$. Se $F=\frac{S^{3}}{G}$, então, pela sequência de homotopia da fibração $p, \pi_{2}(Y) \leqslant \pi_{2}(B) \cong \mathbb{Z}$ e $\pi_{6}(Y)$ é finito 
(pois é tal que $\cdots \mathbb{Z}_{12} \rightarrow \pi_{6}(Y) \rightarrow \mathbb{Z}_{12} \cdots$ é uma sequência exata), donde $\omega^{\prime \prime}$ é o homomorfismo nulo. Se $F=S^{2}$, então, pelo lema 181, $\omega^{\prime \prime} \equiv 0$. Se $F=\mathbb{R} P(2)$, então, pela proposição $182, \omega^{\prime \prime} \equiv 0$. Desta forma, se $Y$ não é um $M$-fibrado sobre $\frac{S^{2}}{G}$, então $\omega^{\prime \prime}$ é o homomorfismo nulo, como queríamos demonstrar.

Observação 189. Seja $p: Y \rightarrow \frac{S^{2}}{G}$ um $M$-fibrado sobre $\frac{S^{2}}{G}$, onde $M \in$ $\left\{S^{1} \times S^{2}, \mathbb{R} P(3) \# \mathbb{R} P(3), S^{1} \tilde{\times} S^{2}, S^{1} \times \mathbb{R} P(2)\right\}$. Neste caso, ainda não conhecemos $\omega^{\prime \prime}: \pi_{6}(Y) \rightarrow \pi_{2}(Y)$, entretanto, usando a sequência de homotopia da fibração p, temos as informações abaixo sobre os grupos envolvidos.

$$
\begin{gathered}
\cdots \pi_{6}(M) \rightarrow \pi_{6}(Y) \stackrel{p_{\#}}{\rightarrow} \pi_{6}\left(\frac{S^{2}}{G}\right) \cdots \\
\cdots \pi_{3}\left(\frac{S^{2}}{G}\right) \rightarrow \pi_{2}(M) \rightarrow \pi_{2}(Y) \stackrel{p_{\#}}{\rightarrow} \pi_{2}\left(\frac{S^{2}}{G}\right) \rightarrow \pi_{1}(M) \rightarrow \pi_{1}(Y) \stackrel{p_{\#}}{\rightarrow} \pi_{1}\left(\frac{S^{2}}{G}\right) \rightarrow 1 .
\end{gathered}
$$

Notemos que, para $i \geqslant 2, \pi_{i}(M) \cong \pi_{i}\left(\mathbb{R} \times S^{2}\right) \cong \pi_{i}\left(S^{2}\right)$, pois $\mathbb{R} \times S^{2}$ reveste $M$, e $\pi_{i}\left(\frac{S^{2}}{G}\right) \cong \pi_{i}\left(S^{2}\right)$, pois $S^{2}$ reveste $\frac{S^{2}}{G}$. Como $\pi_{6}\left(S^{2}\right) \cong \mathbb{Z}_{12}$, então $\pi_{6}(Y)$ é finito. Ainda, $\pi_{2}(Y)$ é tal que

$$
\cdots \mathbb{Z} \rightarrow \mathbb{Z} \rightarrow \pi_{2}(Y) \stackrel{p_{\text {\# }}}{\rightarrow} \mathbb{Z} \rightarrow \pi_{1}(M) \rightarrow \pi_{1}(Y) \stackrel{p_{\text {专 }}}{\rightarrow} \pi_{1}\left(\frac{S^{2}}{G}\right) \rightarrow 1 .
$$

\subsubsection{Variedades de Grassmann}

Seja $G_{k, k+l}$ a variedade de Grassmann de $k$-subespaços vetoriais de $\mathbb{R}^{k+l}$, com $k, l \geqslant 1$. Seja $\widetilde{G}_{k, k+l}$ a variedade de Grassmann de $k$-subespaços vetoriais orientados de $\mathbb{R}^{k+l}$. Consideremos a fibração abaixo

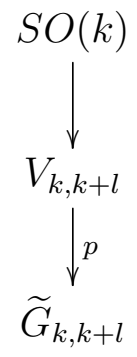


que projeta um $k$-referencial ortonormal em $\mathbb{R}^{k+l}$ no $k$-subespaço vetorial por ele gerado.

Notemos que $V_{k, k+l}$ é $(l-1)$-conexo. Ainda, notemos que $\widetilde{G}_{k, k+l} \approx \widetilde{G}_{l, k+l}$, pois $\widetilde{G}_{k, k+l} \ni W \mapsto W^{\perp} \in \widetilde{G}_{l, k+l}$ é um homeomorfismo. Observemos que $\widetilde{G}_{k, k+l}$ é uma variedade $k l$-dimensional. Assim, estamos interessados em $\omega^{\prime \prime}$ : $\pi_{k l+1}\left(\widetilde{G}_{k, k+l}\right) \rightarrow \pi_{2}\left(\widetilde{G}_{k, k+l}\right)$.

Lema 190. Consideremos os grupos $\pi_{k l+1}\left(\widetilde{G}_{k, k+l}\right), \pi_{2}\left(\widetilde{G}_{k, k+l}\right)$, com $k, l \geqslant 2$. Se $k, l \geqslant 3$, então ambos os grupos são finitos, enquanto que se $k=2 \not \equiv l(2)$, ou $l=2 \not \equiv k(2)$, então ambos são infinitos. Se $k=2 \equiv l(2)$, ou $l=2 \equiv k(2)$, então $\pi_{k l+1}\left(\widetilde{G}_{k, k+l}\right)$ é finito e $\pi_{2}\left(\widetilde{G}_{k, k+l}\right)$ é infinito livre de torção.

Demonstração. Com efeito, consideremos a sequência de homotopia da fibração $p$,

$$
\begin{gathered}
\cdots \pi_{i+1}\left(\widetilde{G}_{k, k+l}\right) \rightarrow \pi_{i}(S O(k)) \rightarrow \pi_{i}\left(V_{k, k+l}\right) \rightarrow \pi_{i}\left(\widetilde{G}_{k, k+l}\right) \rightarrow \pi_{i-1}(S O(k)) \cdots \\
\cdots \pi_{2}\left(V_{k, k+l}\right) \rightarrow \pi_{2}\left(\widetilde{G}_{k, k+l}\right) \rightarrow \pi_{1}(S O(k)) \rightarrow \pi_{1}\left(V_{k, k+l}\right) \cdots
\end{gathered}
$$

Primeiramente, analisemos o segundo grupo de homotopia de $\widetilde{G}_{k, k+l}$. Por um lado, notemos que $V_{k, k+l}$ é 1 -conexo (pois $l \geqslant 2$ ). Por outro lado, notemos que $\pi_{2}(S O(k))=0\left(\right.$ pois $\pi_{2}(S O(2)) \cong \pi_{2}\left(S^{1}\right)=0, \pi_{2}(S O(3)) \cong \pi_{2}(\mathbb{R} P(3))=0$ e $S O(k) \rightarrow S O(k+1) \rightarrow S^{k}$ é fibração). Daí, a sequência

$$
1 \rightarrow \pi_{2}\left(V_{k, k+l}\right) \rightarrow \pi_{2}\left(\widetilde{G}_{k, k+l}\right) \rightarrow \pi_{1}(S O(k)) \rightarrow 1
$$

é exata curta. Se $k=2=l$, então $\pi_{2}\left(\widetilde{G}_{2,4}\right) \cong \mathbb{Z} \oplus \mathbb{Z}\left(\right.$ pois $\pi_{1}(S O(2)) \cong \mathbb{Z}$ e $\left.\pi_{2}\left(V_{2,4}\right) \cong \pi_{2}\left(V_{1,3}\right) \cong \mathbb{Z}\right)$. Seja $l \geqslant 3$. Então $V_{2,2+l}$ é 2 -conexo. Se $k=2$, segue que $\pi_{2}\left(\widetilde{G}_{2,2+l}\right) \cong \pi_{1}(S O(2)) \cong \mathbb{Z}$. Se $k \geqslant 3$, então $\pi_{2}\left(\widetilde{G}_{k, k+l}\right) \cong \pi_{1}(S O(k)) \cong$ $\pi_{1}(S O(3)) \cong \mathbb{Z}_{2}$. Analisemos, agora, o $(k l+1)$-ésimo grupo de homotopia de $\widetilde{G}_{k, k+l}$. Consideremos o seguinte trecho da sequência de homotopia da fibração $p$ : 


$$
\cdots \pi_{k l+1}(S O(k)) \rightarrow \pi_{k l+1}\left(V_{k, k+l}\right) \rightarrow \pi_{k l+1}\left(\widetilde{G}_{k, k+l}\right) \rightarrow \pi_{k l}(S O(k)) \cdots
$$

Consideremos, para todo $k \geqslant 2$, a fibração abaixo.

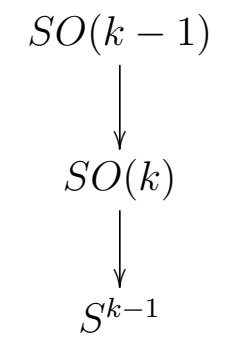

Afirmamos que $\pi_{k l+1}(S O(k))$ e $\pi_{k l}(S O(k))$ são finitos, para todo $k, l \geqslant 2$. De fato, observemos que $k l+1>k l>2(k-1)-1$ e $k l+1>k l>k-1$, com $k, l \geqslant 2$. Notemos ainda que a base da fibração acima é $S^{k-1}$, a base da fibração construída da mesma forma para a fibra da fibração acima é $S^{k-2}$, a base da fibração construída do mesmo modo para a fibra da fibração anterior é $S^{k-3}$ e assim por diante. Desta forma, como a esfera de maior dimensão que aparece neste estudo é $S^{k-1}$, então o resultado segue, das desigualdades anteriores, por [Se-51]. Portanto, notemos que $\pi_{k l+1}\left(\widetilde{G}_{k, k+l}\right)$ é finito se, e só se, $\pi_{k l+1}\left(V_{k, k+l}\right)$ é finito. Consideremos agora, para todo $k, l \geqslant 2$, a fibração abaixo.

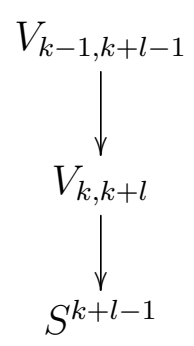

Afirmamos que $\pi_{k l+1}\left(V_{k, k+l}\right)$ é finito para $k, l \geqslant 3$ e para $k=2 \equiv l(2)$, e é infinito para $k=2 \not \equiv l(2)$. Com efeito, notemos que a base da fibração acima é $S^{k+l-1}$, a base da fibração construída da mesma forma para a fibra da fibração acima é $S^{k+l-2}$, a base da fibração construída do mesmo modo para a fibra da fibração anterior é $S^{k+l-3}$ e assim por diante. Assim, as esferas 
envolvidas nesta análise são da forma $S^{k+l-j}$, com $j \geqslant 1$. Observemos que, para $j \geqslant 1,(k l+1)-(k+l-j)=(l-1)(k-1)+j$ é sempre diferente de zero e $(k l+1)-(2(k+l-j)-1)=(l-2)(k-2)+2(j-1)$ é igual a zero se, e só se, $l=2$ ou $k=2$, e $j=1$. Logo, para $k, l \geqslant 3$, temos, por [Se-51], que $\pi_{k l+1}\left(S^{k+l-j}\right)$ é finito, para todo $j \geqslant 1$, e portanto a primeira parte da afirmação segue. Seja $k=2$. Então a fibração acima assume a forma $S^{l} \rightarrow V_{2,2+l} \rightarrow S^{l+1}$. Notemos, por [Se-51], que $\pi_{2 l}\left(S^{l}\right), \pi_{2 l+1}\left(S^{l}\right)$ são sempre finitos e $\pi_{2 l+1}\left(S^{l+1}\right)$ é infinito se, e só se, $l+1$ é par, donde a segunda parte da afirmação segue para $k=2$, usando a sequência de homotopia da fibração. Desde que $\widetilde{G}_{k, k+l} \approx \widetilde{G}_{l, k+l}$, o resultado também segue para $l=2$.

Proposição 191. Seja $\widetilde{G}_{k, k+l}$ a variedade de Grassmann de k-subespaços vetoriais orientados de $\mathbb{R}^{k+l}$, com $k, l \geqslant 1$. Se $k=1$, ou $l=1$, ou $k=2 \equiv$ $l(2)$, ou $l=2 \equiv k(2)$, então $\omega^{\prime \prime} \equiv 0$, exceto para $\widetilde{G}_{2,3} \approx \widetilde{G}_{1,3} \approx S^{2}$ quando $\omega^{\prime \prime}=\times( \pm 1)$.

Demonstração. Com efeito, desde que $\widetilde{G}_{k, k+l} \approx \widetilde{G}_{l, k+l}$, basta analisarmos quando $l=1$ e quando $l=2 \equiv k(2)$. Se $l=1$, então $\widetilde{G}_{k, k+1} \approx \widetilde{G}_{1, k+1} \approx S^{k} \mathrm{e}$ portanto, pela proposição 151 , $\omega^{\prime \prime} \equiv 0$, exceto para $\widetilde{G}_{2,3} \approx \widetilde{G}_{1,3} \approx S^{2}$ quando $\omega^{\prime \prime}=\times( \pm 1)$. Se $l=2 \equiv k(2)$, então, pelo lema anterior, $\pi_{k l+1}\left(\widetilde{G}_{k, k+l}\right)$ é finito e $\pi_{2}\left(\widetilde{G}_{k, k+l}\right)$ é infinito livre de torção. Logo, $\omega^{\prime \prime} \equiv 0$.

Desta forma, na maioria dos casos, ainda não conhecemos $\omega^{\prime \prime}$. 


\section{Capítulo 3}

\section{Raízes em codimensão 1, sendo o domínio uma 3-variedade}

Em todo o capítulo, seja $f: X^{3} \rightarrow Y^{2}$ uma aplicação contínua, onde $X, Y$ são variedades fechadas, conexas e seja $y_{0} \in Y$.

Neste capítulo, estamos interessados em estudar, para o par $\left(y_{0}, f\right)$, quando o número mínimo de componentes de coincidências $M C C\left(y_{0}, f\right)$ é igual ao número de Nielsen $\tilde{N}\left(y_{0}, f\right)$, isto é, queremos saber quando o par $\left(y_{0}, f\right)$ satisfaz a propriedade de Wecken, supondo que o domínio $X$ de $f$ é uma 3variedade fechada, conexa e o contradomínio $Y$ de $f$ é uma superfície fechada, conexa. Sabemos que $X$ ou tem grupo fundamental finito e é revestido por $S^{3}$ (que são as formas espaciais esféricas), ou é $K(\pi, 1)$ e é revestido por $\mathbb{R}^{3}$, ou pertence ao conjunto $\left\{S^{1} \times S^{2}, \mathbb{R} P(3) \# \mathbb{R} P(3), S^{1} \tilde{\times} S^{2}, S^{1} \times \mathbb{R} P(2)\right\}$ e é revestido por $\mathbb{R} \times S^{2}$. Da mesma forma, sabemos que $Y$ ou tem grupo fundamental finito e é revestido por $S^{2}$ (que são $S^{2}$ e $\mathbb{R} P(2)$ ), ou é $K(\pi, 1$ ) e é revestido por $\mathbb{R}^{2}$ (que são as superfícies fechadas com característica de Euler não positiva). Mais especificamente, neste capítulo, estudamos a validade da propriedade de Wecken ora quando $X$ é uma forma espacial esférica qualquer 
e $Y=K(\pi, 1)$, ora quando $X=\mathbb{R} P(3)$ e $Y=S^{2}$. No primeiro caso, nós obtemos que $\operatorname{MCC}\left(y_{0}, f\right)=N\left(y_{0}, f\right)=0$ sempre (proposição 192). No segundo caso, nós inicialmente construimos algumas aplicações contínuas de $\mathbb{R} P(3)$ em $S^{2}$ e as enumeramos. Em seguida, nós obtemos que $\left[\mathbb{R} P(3), S^{2}\right]$ é uma união disjunta de duas famílias enumeráveis que são completamente determinadas pelas aplicações contínuas construídas (teorema 196). Daí, para cada família, nós determinamos o grupo de bordismo (proposições 198, 199 e 201) e nós analisamos o anulamento de $\tilde{\omega}\left(y_{0}, f\right)$ (proposições 203 e 204 e teorema 205). Por fim, nós obtemos que $M C C\left(y_{0}, f\right)=N\left(y_{0}, f\right)=1$ se $f$ não é homotópica a aplicação constante e $M C C\left(y_{0}, f\right)=N\left(y_{0}, f\right)=0$, caso contrário (teorema 206).

\section{$3.1 \quad X$ uma forma espacial esférica e $\chi(Y) \leqslant 0$}

Sejam $f: \frac{S^{3}}{G} \rightarrow Y$ uma aplicação contínua e $y_{0} \in S^{2}$, onde $\frac{S^{3}}{G}$ denota uma forma espacial esférica dada por uma ação livre de $G$ em $S^{3}$, e onde supomos que a característica de Euler de $Y, \chi(Y)$, é não positiva.

O resultado abaixo nos diz em particular que, neste caso, todo par $\left(y_{0}, f\right)$ satisfaz a propriedade de Wecken.

Proposição 192. Sejam $f: \frac{S^{3}}{G} \rightarrow Y$ uma aplicação contínua e $y_{0} \in S^{2}$, com $\chi(Y) \leqslant 0$. Então $\left(y_{0}, f\right)$ é deformável a livre de coincidência. Em particular, $M C C\left(y_{0}, f\right)=0=\tilde{N}\left(y_{0}, f\right)$.

Demonstração. De fato, pelo teorema de classificação de superfícies fechadas, $\chi(Y) \leqslant 0$ se, e somente se, $\pi_{1}(Y)$ é infinito. Ainda, temos que $\pi_{1}\left(\frac{S^{3}}{G}\right) \cong G$ é finito. Logo, \# $\pi_{0}\left(E\left(y_{0}, f\right)\right)=\left[\pi_{1}(Y): f_{\#}\left(\pi_{1}\left(\frac{S^{3}}{G}\right)\right)\right]$ é infinito (igual a $\# \pi_{1}(Y)$, pois $f_{\#}\left(\pi_{1}\left(\frac{S^{3}}{G}\right)\right)=1$ uma vez que $\pi_{1}(Y)$ é livre de torção) 
e portanto, por [GoWo-05], $\left(y_{0}, f\right)$ é deformável a livre de coincidência. Em particular, pelo lema $120, \operatorname{MCC}\left(y_{0}, f\right)=0=\tilde{N}\left(y_{0}, f\right)$.

Desta forma, quando $X=\frac{S^{3}}{G}$, resta analisar a validade da propriedade de Wecken apenas para $Y=S^{2}$ e para $Y=\mathbb{R} P(2)$. Na próxima seção, vamos fazer isto para $X=\mathbb{R} P(3)$ e $Y=S^{2}$.

\section{$3.2 \quad X=\mathbb{R} P(3)$ e $Y=S^{2}$}

Em toda a seção, sejam $f: \mathbb{R} P(3) \rightarrow S^{2}$ uma aplicação contínua e $y_{0} \in S^{2}$.

Nesta seção, vamos inicialmente construir algumas aplicações contínuas de $\mathbb{R} P(3)$ em $S^{2}$ e enumerá-las e vamos determinar $\left[\mathbb{R} P(3), S^{2}\right]$. Em seguida, para estas aplicações, vamos calcular os grupos envolvidos, analisar o anulamento de $\tilde{\omega}\left(y_{0}, f\right)$ e determinar $M C C\left(y_{0}, f\right)$ e $\tilde{N}\left(y_{0}, f\right)$.

Notemos que, como o contradomínio de $f$ é $S^{2}$, então $f$ é deformável a livre de raiz se, e só se, $f$ é homotópica a aplicação constante. Em particular, se $f \not y_{0}$, segue que $M C C\left(y_{0}, f\right) \geqslant 1$ e, se $f \simeq y_{0}$, temos que $M C C\left(y_{0}, f\right)=$ $0=\tilde{N}\left(y_{0}, f\right)$.

\subsubsection{Particularidades sobre o invariante}

Consideremos o seguinte diagrama geométrico

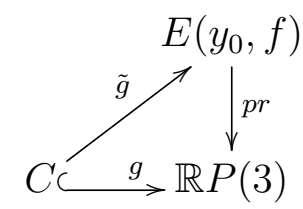

onde $C:=\operatorname{Coin}\left(y_{0}, f\right)=f^{-1}\left(y_{0}\right), E\left(y_{0}, f\right)=\left\{(x, \theta) \in \mathbb{R} P(3) \times\left(S^{2}\right)^{I} \mid \theta(0)=\right.$ $\left.y_{0}, \theta(1)=f(x)\right\}, \tilde{g}(x)=\left(x, y_{0}\right)$ e $\operatorname{pr}(x, \theta)=x$. Notemos que $p r$ é uma fibração tal que $p r^{-1}(g(x)) \approx \Omega\left(S^{2}, y_{0}\right)$. Consideremos a sequência de homotopia da fibração $p r$, 
$\cdots \pi_{2}(\mathbb{R} P(3)) \rightarrow \pi_{1}\left(\Omega\left(S^{2}, y_{0}\right)\right) \rightarrow \pi_{1}\left(E\left(y_{0}, f\right)\right) \rightarrow \pi_{1}(\mathbb{R} P(3)) \rightarrow \pi_{0}\left(\Omega\left(S^{2}, y_{0}\right)\right) \cdots$

Desde que $\pi_{2}(\mathbb{R} P(3)) \cong \pi_{2}\left(S^{3}\right)=1, \pi_{1}(\mathbb{R} P(3)) \cong \mathbb{Z}_{2}, \pi_{1}\left(\Omega\left(S^{2}, y_{0}\right)\right) \cong$ $\pi_{2}\left(S^{2}\right) \cong \mathbb{Z}$ e $\pi_{0}\left(\Omega\left(S^{2}, y_{0}\right)\right) \approx \pi_{1}\left(S^{2}\right)=1$, então

$$
1 \rightarrow \mathbb{Z} \rightarrow \pi_{1}\left(E\left(y_{0}, f\right)\right) \rightarrow \mathbb{Z}_{2} \rightarrow 1
$$

é uma sequência exata curta. Em particular, $\pi_{1}\left(E\left(y_{0}, f\right)\right) \in\left\{\mathbb{Z}, \mathbb{Z} \oplus \mathbb{Z}_{2}, \mathbb{Z}_{2} *\right.$ $\left.\mathbb{Z}_{2}\right\}$. Ainda, $\pi_{0}\left(E\left(y_{0}, f\right)\right) \approx \frac{\pi_{1}\left(S^{2}\right)}{\sim}=\frac{\pi_{1}\left(S^{2}\right)}{\operatorname{Im} f_{\#}}=1$, donde $E\left(y_{0}, f\right)$ é conexo por caminhos e portanto $R\left(y_{0}, f\right)=1$, isto é, $\left(y_{0}, f\right)$ tem apenas uma classe de Reidemeister. Notemos, agora, que o invariante $\tilde{\omega}$ é tal que

$$
\tilde{\omega}\left(y_{0}, f\right):=[C, \tilde{g}, \bar{g}] \in \Omega_{1}\left(E\left(y_{0}, f\right) ; p r^{*}\left(y_{0}^{*}\left(\tau_{S^{2}}\right)-\tau_{\mathbb{R} P(3)}\right)\right)=\Omega_{1}^{f r}\left(E\left(y_{0}, f\right)\right),
$$

pois $\mathbb{R} P(3)$ é paralelizável.

Observação 193. Desde que pr é fibração e $\Omega\left(S^{2}, y_{0}\right)$ e $\mathbb{R} P(3)$ são $C W$ complexos, então E $\left(y_{0}, f\right)$ também o é (vide [FrPi-90], páginas 236 e 238). Ainda, como $\pi_{n}\left(S^{2}\right) \cong \pi_{n-1}\left(\Omega\left(S^{2}, y_{0}\right)\right)$ e $\pi_{n}(\mathbb{R} P(3))$ são finitamente gerados, para todo $n \geqslant 1$, segue que $\pi_{n}\left(E\left(y_{0}, f\right)\right)$ é finitamente gerado, para todo $n \geqslant 1$.

Agora, a fim de estudar o invariante $\tilde{\omega}\left(y_{0}, f\right)$ e o grupo em que ele se encontra, vamos, na próxima subseção, analisar o conjunto $\left[\mathbb{R} P(3), S^{2}\right]$.

\subsubsection{Aplicações contínuas de $\mathbb{R} P(3)$ em $S^{2}$}

Nesta subseção, vamos estudar aplicações contínuas de $\mathbb{R} P(3)$ em $S^{2}$. Consideremos a projeção natural $p_{\mathbb{R} P(3)}: S^{3} \rightarrow \mathbb{R} P(3)$. Vamos apresentar, para cada $m \in \mathbb{Z}$, uma aplicação contínua $f^{\prime}: \mathbb{R} P(3) \rightarrow S^{2}$ tal que $f^{\prime} \circ p_{\mathbb{R} P(3)} \simeq$ $m h$, onde $h: S^{3} \rightarrow S^{2}$ denota a aplicação de Hopf. Em outras palavras, para cada $m \in \mathbb{Z}$, vamos exibir $f^{\prime}$ que, a menos de homotopia, fatora $m h$ via 
$p_{\mathbb{R} P(3)}$, isto é, tal que o seguinte diagrama geométrico é homotópico comutativo:

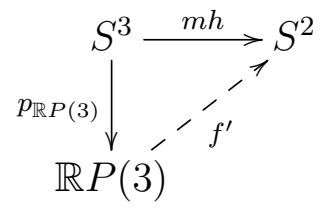

\section{Caso I - m ímpar.}

Sabemos que $\pi_{3}\left(S^{2}\right) \cong \mathbb{Z}$ e que a aplicação de Hopf $h: S^{3} \rightarrow S^{2}$ representa um gerador de $\pi_{3}\left(S^{2}\right)$. Se $h$ é dada por

$$
\mathbb{C} \times \mathbb{C} \supset S^{3} \ni\left(z_{1}, z_{2}\right) \mapsto S^{1}\left(z_{1}, z_{2}\right) \in \frac{S^{3}}{S^{1}},
$$

onde $S^{1}\left(z_{1}, z_{2}\right)$ representa a órbita de $\left(z_{1}, z_{2}\right)$ dada pela ação

$$
S^{1} \times(\mathbb{C} \times \mathbb{C}) \ni\left(\lambda,\left(z_{1}, z_{2}\right)\right) \mapsto\left(\lambda z_{1}, \lambda z_{2}\right) \in \mathbb{C} \times \mathbb{C}
$$

então definindo $h^{\prime}$ por $\left[z_{1}, z_{2}\right] \mapsto S^{1}\left(z_{1}, z_{2}\right)$, temos que $h^{\prime}$ fatora $h$ via $p_{\mathbb{R} P(3)}$, conforme o diagrama geométrico abaixo.

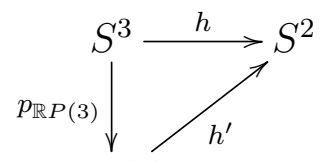

$\mathbb{R} P(3)$

Seja $n \in \mathbb{Z}$. Agora, se $a_{2 n+1}: S^{3} \rightarrow S^{3}$ é uma aplicação de grau $2 n+1$, então existe $a_{2 n+1}^{\prime}: \mathbb{R} P(3) \rightarrow \mathbb{R} P(3)$ tal que $p_{\mathbb{R} P(3)} \circ a_{2 n+1}=a_{2 n+1}^{\prime} \circ p_{\mathbb{R} P(3)}$ e portanto $h^{\prime} \circ a_{2 n+1}^{\prime}$ fatora $h \circ a_{2 n+1} \simeq(2 n+1) h$ via $p_{\mathbb{R} P(3)}$, conforme o diagrama geométrico abaixo.

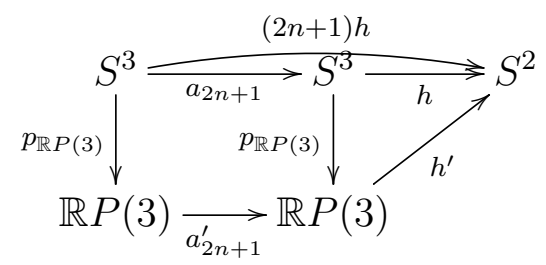


Observação 194. Sobre $M C C\left(y_{0}, h^{\prime} \circ a_{2 n+1}^{\prime}\right)$ :

Seja $a_{2 n+1}: S^{3} \rightarrow S^{3}$ dada por $a_{2 n+1}\left(z_{1}, z_{2}\right)=\left(z_{1}{ }^{2 n+1}, z_{2}\right)$, para todo $\left(z_{1}, z_{2}\right) \in S^{3} \subset \mathbb{C} \times \mathbb{C}$. Daí, $a_{2 n+1}^{\prime}: \mathbb{R} P(3) \rightarrow \mathbb{R} P(3)$ é tal que $a_{2 n+1}^{\prime}\left(\left[z_{1}, z_{2}\right]\right)=$ $\left[z_{1}^{2 n+1}, z_{2}\right]$, pois $a_{2 n+1}\left(-z_{1},-z_{2}\right)=\left(\left(-z_{1}\right)^{2 n+1},-z_{2}\right)=-a_{2 n+1}\left(z_{1}, z_{2}\right)$. Notemos que ambas tem grau $2 n+1$, pois $a_{2 n+1}^{-1}(1,0)=\left\{\mathrm{e}^{\frac{2 \pi i k}{2 n+1}} \mid 0 \leqslant k \leqslant\right.$ $2 n\}$. Como $h^{-1}\left(S^{1}(0,1)\right)=0_{\mathbb{C}} \times S^{1}$ e $a_{2 n+1}^{-1}\left(0_{\mathbb{C}} \times S^{1}\right)=0_{\mathbb{C}} \times S^{1}$, então $\left(h \circ a_{2 n+1}\right)^{-1}\left(S^{1}(0,1)\right)=0_{\mathbb{C}} \times S^{1}$ e portanto $\left(h^{\prime} \circ a_{2 n+1}^{\prime}\right)^{-1}\left(S^{1}(0,1)\right) \approx 0_{\mathbb{C}} \times S^{1}$ é conexo por caminhos. Em particular, $M C C\left(y_{0}, h^{\prime} \circ a_{2 n+1}^{\prime}\right) \leqslant 1$.

\section{Caso II - m par.}

Consideremos uma bola aberta $B^{3} \subset \mathbb{R} P(3)$ e consideremos a aplicação colapsante $k_{3}: \mathbb{R} P(3) \rightarrow \frac{\mathbb{R} P(3)}{\mathbb{R} P(3) \backslash B^{3}}$. Identificando $\frac{\mathbb{R} P(3)}{\mathbb{R} P(3) \backslash B^{3}}$ com $S^{3}$, obtemos que $k_{3}$ é uma aplicação contínua de $\mathbb{R} P(3)$ em $S^{3}$. Agora, pelo teorema de classificação de Hopf-Whitney (vide [Whg-78], página 244), segue que $\left[\mathbb{R} P^{3}, S^{3}\right] \approx H^{3}(\mathbb{R} P(3), \mathbb{Z})$, o qual, por sua vez, é isomorfo a $\mathbb{Z}$, pois $\mathbb{R} P(3)$ é orientável. Usando esta identificação, notemos que $k_{3}$ representa um gerador de $\left[\mathbb{R} P^{3}, S^{3}\right]$. Ainda, observemos que $a_{2}:=k_{3} \circ p_{\mathbb{R} P(3)}$ é uma aplicação de grau 2. Assim, $h \circ k_{3}$ fatora $h \circ a_{2} \simeq 2 h$ via $p_{\mathbb{R} P(3)}$, conforme o diagrama geométrico abaixo.

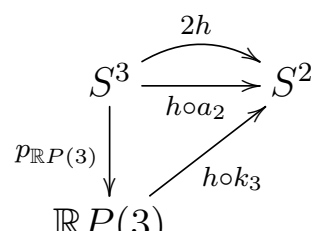

$\mathbb{R} P(3)$

Seja $n \in \mathbb{Z}$. Na verdade, $(n h) \circ k_{3}$ fatora $(n h) \circ a_{2} \simeq(2 n) h$ via $p_{\mathbb{R} P(3)}$, conforme o diagrama geométrico abaixo. 


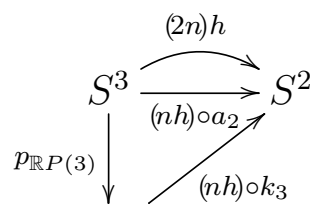

$\mathbb{R} P(3)$

Observação 195. Sobre $M C C\left(y_{0},(n h) \circ k_{3}\right)$ :

Seja $n \neq 0$. Desde que $M C C\left(y_{0}, n h\right)=1$, então, via bordismo, identificamos $(n h)^{-1}\left(y_{0}\right) \operatorname{com} S^{1}$. Daí, identificamos $\left((n h) \circ k_{3}\right)^{-1}\left(y_{0}\right) \operatorname{com} k_{3}^{-1}\left(S^{1}\right)$. Da construção da aplicação colapsante $k_{3}$, se $S^{1} \cap\left(\mathbb{R} P(3) \backslash B^{3}\right)=\emptyset$, então $k_{3}^{-1}\left(S^{1}\right) \approx S^{1}$ e, caso contrário, $k_{3}^{-1}\left(S^{1}\right) \approx S^{1} \vee\left(\mathbb{R} P(3) \backslash B^{3}\right)$. De qualquer forma, $\left((n h) \circ k_{3}\right)^{-1}\left(y_{0}\right)$ é conexo por caminhos. Daí, $M C C\left(y_{0},(n h) \circ k_{3}\right) \leqslant 1$.

Classificação de aplicações de $\mathbb{R} P(3)$ em $S^{2}$.

Consideremos a projeção natural $p_{\mathbb{R} P(2)}: S^{2} \rightarrow \mathbb{R} P(2)$. Consideremos a sequência de Puppe associada à aplicação contínua $p_{\mathbb{R} P(2)}$,

$$
S^{2} \rightarrow \mathbb{R} P(2) \rightarrow \mathbb{R} P(3) \rightarrow \sum S^{2} \rightarrow \sum \mathbb{R} P(2) \rightarrow \sum \mathbb{R} P(3) \rightarrow \cdots
$$

A partir desta sequência, usando que $\sum S^{2} \approx S^{3}$, consideremos a sequência exata longa (para classes de homotopia de aplicações em $S^{2}$ ),

$$
\cdots \rightarrow\left[\sum \mathbb{R} P^{2}, S^{2}\right] \rightarrow\left[S^{3}, S^{2}\right] \stackrel{k_{3}^{*}}{\rightarrow}\left[\mathbb{R} P^{3}, S^{2}\right] \stackrel{i^{*}}{\rightarrow}\left[\mathbb{R} P^{2}, S^{2}\right] \stackrel{p_{\mathbb{R} P(2)}^{*}}{\rightarrow}\left[S^{2}, S^{2}\right],
$$

onde $i: \mathbb{R} P(2) \hookrightarrow \mathbb{R} P(3)$ é a inclusão. Observemos que, em geral, a parte final desta sequência exata longa não é formada por grupos. Notemos que $\left[S^{3}, S^{2}\right] \cong \pi_{3}\left(S^{2}\right) \cong \mathbb{Z}$ e $\left[S^{2}, S^{2}\right] \cong \pi_{2}\left(S^{2}\right) \cong \mathbb{Z}$. Ainda, ao considerarmos a sequência exata longa (para classes de homotopia de aplicações em $S^{2}$ ) dada pela sequência de Puppe associada à projeção natural $p_{\mathbb{R} P(1)}: S^{1} \rightarrow \mathbb{R} P(1)$, obtemos que $\left[\sum \mathbb{R} P^{2}, S^{2}\right] \cong \mathbb{Z}_{2}$. Consideremos uma bola aberta $B^{2} \subset \mathbb{R} P(2)$ e consideremos a aplicação colapsante $k_{2}: \mathbb{R} P(2) \rightarrow \frac{\mathbb{R} P(2)}{\mathbb{R} P(2) \backslash B^{2}}$. Pelo teorema de classificação de Hopf-Whitney (vide [Whg-78], página 244), segue que 
$\left[\mathbb{R} P^{2}, S^{2}\right] \approx H^{2}(\mathbb{R} P(2), \mathbb{Z})$, o qual, por sua vez, é isomorfo a $\mathbb{Z}_{2}$, uma vez que $\mathbb{R} P(2)$ é não orientável. Usando esta identificação, observemos que $k_{2}$ representa um gerador de $\left[\mathbb{R} P^{2}, S^{2}\right]$ e, desta forma, $\left[\mathbb{R} P^{2}, S^{2}\right]=\left\{0,\left[k_{2}\right]\right\}$, onde 0 denota a classe de homotopia da aplicação constante $c: \mathbb{R} P(2) \rightarrow S^{2}$. Assim, obtemos a sequência exata (de conjuntos) abaixo.

$$
\cdots \rightarrow \mathbb{Z}_{2} \stackrel{0}{\rightarrow} \mathbb{Z} \stackrel{k_{3}^{*}}{\rightarrow}\left[\mathbb{R} P^{3}, S^{2}\right] \stackrel{i^{*}}{\rightarrow}\left\{0,\left[k_{2}\right]\right\} \stackrel{p_{\mathbb{R} P(2)}^{*}}{\rightarrow} \mathbb{Z} .
$$

Desde que $c \circ p_{\mathbb{R} P(2)}$ e $k_{2} \circ p_{\mathbb{R} P(2)}$ são nulo-homotópicas (pois as induzidas no segundo grupo de homologia com coeficientes em $\mathbb{Z}$ são nulas), então $p_{\mathbb{R} P(2)}^{*}$ é a aplicação nula. Logo,

$$
1 \rightarrow\left[S^{3}, S^{2}\right] \stackrel{k_{3}^{*}}{\rightarrow}\left[\mathbb{R} P(3), S^{2}\right] \stackrel{i^{*}}{\rightarrow}\left[\mathbb{R} P(2), S^{2}\right] \rightarrow 1
$$

é uma sequência exata curta (de conjuntos). Notemos que $\left[\mathbb{R} P(3), S^{2}\right]=$ $i^{*-1}(0) \dot{\cup} i^{*-1}\left(\left[k_{2}\right]\right)$ e $i^{*-1}(0)=\operatorname{Im}\left(k_{3}^{*}\right)$. Como $\pi_{3}\left(S^{2}\right)=<h>$, então $i^{*-1}(0)=$ $\operatorname{Im}\left(k_{3}^{*}\right)=\left\{\left[(n h) \circ k_{3}\right] \mid n \in \mathbb{Z}\right\}$. Vamos, agora, determinar $i^{*-1}\left(\left[k_{2}\right]\right)$. Afirma$\operatorname{mos}$ que $\left[h^{\prime} \circ a_{2 n+1}^{\prime}\right] \in i^{*-1}\left(\left[k_{2}\right]\right)$, para todo $n \in \mathbb{Z}$. De fato, suponhamos, por absurdo, que $h^{\prime} \circ a_{2 n+1}^{\prime} \simeq(m h) \circ k_{3}$, para algum $m \in \mathbb{Z}$. Daí,

$$
(2 n+1) h \simeq h^{\prime} \circ a_{2 n+1}^{\prime} \circ p_{\mathbb{R} P(3)} \simeq(m h) \circ k_{3} \circ p_{\mathbb{R} P(3)} \simeq(2 m) h
$$

o que não ocorre. Vamos verificar que $i^{*-1}\left(\left[k_{2}\right]\right)=\left\{\left[h^{\prime} \circ a_{2 n+1}^{\prime}\right] \mid n \in \mathbb{Z}\right\}$.

Seja $B_{r} \subset \mathbb{R}^{3}$ a bola aberta de centro 0 e raio $r$ e seja $S_{r}=\partial B_{r}$, com $r>0$. Seja ainda $\overline{B_{r}} \subset \mathbb{R}^{3}$ a bola fechada de centro 0 e raio $r$. Consideremos as identificações $\mathbb{R} P(3)=\frac{\mathbb{R} P(3) \backslash B_{1}}{S_{1}}$ e $S^{3}=\frac{\overline{B_{1}}}{S_{1}}$. Associada à sequência de Puppe, temos uma ação

$$
\begin{array}{ccc}
\pi_{3}\left(S^{2}\right) \times\left[\mathbb{R} P(3), S^{2}\right] & \stackrel{\Theta}{\rightarrow} & {\left[\mathbb{R} P(3), S^{2}\right]} \\
([g],[f]) & \mapsto & {[\psi \circ(f \vee g) \circ \varphi]}
\end{array}
$$

onde a composta $R P(3) \stackrel{\varphi}{\rightarrow} R P(3) \vee S^{3} \stackrel{f \vee g}{\rightarrow} S^{2} \vee S^{2} \stackrel{\psi}{\rightarrow} S^{2}$ é tal que 


$$
\begin{aligned}
\varphi: x \mapsto \begin{cases}(\bar{x}, \infty), & \text { se } x \in \mathbb{R} P(3) \backslash B_{1}, \\
(\infty, \bar{x}), & \text { se } x \in \overline{B_{1}},\end{cases} \\
f \vee g:\left\{\begin{array}{l}
(x, \infty) \mapsto(f(x), \infty), \\
(\infty, x) \mapsto(\infty, g(x)),
\end{array}\right. \\
\psi:\left\{\begin{array}{ll}
(x, \infty) \\
(\infty, x)
\end{array} \mapsto x,\right.
\end{aligned}
$$

e onde $\infty$ denota o ponto de colagem das somas wedge. Por esta ação, temos que a órbita de um elemento de $i^{*-1}\left(\left[k_{2}\right]\right)$ é igual a $i^{*-1}\left(\left[k_{2}\right]\right.$ ) (e também que a órbita de um elemento de $i^{*-1}(0)$ é igual a $\left.i^{*-1}(0)\right)$. Assim, a fim de determinar $i^{*-1}\left(\left[k_{2}\right]\right)$, basta calcularmos a órbita de $\left[h^{\prime}\right] \in i^{*-1}\left(\left[k_{2}\right]\right)$. Vamos calcular $\Theta\left([h],\left[h^{\prime}\right]\right)$. Para isto, vamos analisar $\left(\psi \circ\left(h^{\prime} \vee h\right) \circ \varphi\right) \circ p_{\mathbb{R} P(3)}$. Consideremos o revestimento universal de $R P(3) \vee S^{3}, q: S^{3} \vee S^{3} \vee S^{3} \rightarrow$ $\mathbb{R} P(3) \vee S^{3}$, tal que

$$
q:\left\{\begin{aligned}
(x, \infty, \infty) & \mapsto(\infty, x) \\
(\infty, x, \infty) & \mapsto\left(p_{\mathbb{R} P(3)}(x), \infty\right) \\
(\infty, \infty, x) & \mapsto(\infty, x)
\end{aligned}\right.
$$

Notemos que $\varphi \circ p_{\mathbb{R} P(3)}$ admite um levantamento $\varphi^{\prime}$. Se $\mathbb{R} P(3)=\frac{\overline{B_{2}}}{x \sim{S_{2}}_{2}-x} \mathrm{e}$ $S^{3}=\frac{S^{2} \times[-2,2]}{S^{2} \times\{-2\}, S^{2} \times\{2\}}$, podemos escrever $p_{\mathbb{R} P(3)}^{-1}\left(\overline{B_{1}}\right)=\overline{B_{1-}} \dot{\cup} \overline{B_{1+}}$, onde $\overline{B_{1-}}:=$ $\frac{S^{2} \times[-2,-1]}{S^{2} \times\{-2\}, S^{2} \times\{2\}}$ e $\overline{B_{1+}}:=\frac{S^{2} \times[1,2]}{S^{2} \times\{-2\}, S^{2} \times\{2\}}$. Consideremos $\varphi^{\prime}: S^{3} \rightarrow S^{3} \vee S^{3} \vee S^{3}$ tal que

$$
\varphi^{\prime}: x \mapsto \begin{cases}(\bar{x}, \infty, \infty), & \text { se } x \in \overline{B_{1+}}, \\ (\infty, \bar{x}, \infty), & \text { se } x \in S^{3} \backslash\left(B_{1+} \cup B_{1-}\right), \\ (\infty, \infty, \bar{x}), & \text { se } x \in \overline{B_{1-}},\end{cases}
$$

Assim, $\varphi \circ p_{\mathbb{R} P(3)}=q \circ \varphi^{\prime}$. Consideremos o seguinte diagrama geométrico

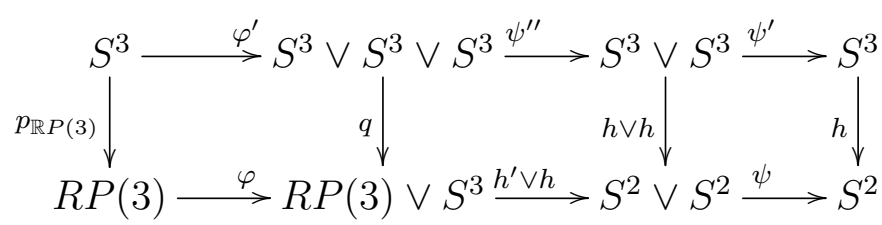


onde

$$
\begin{aligned}
\psi^{\prime \prime}:\left\{\begin{array}{rll}
(x, \infty, \infty) & \mapsto(\infty, x), \\
(\infty, x, \infty) & \mapsto(x, \infty), \\
(\infty, \infty, x) & \mapsto(\infty, x),
\end{array}\right. \\
\psi^{\prime}:\left\{\begin{aligned}
(x, \infty) & \mapsto x . \\
(\infty, x) &
\end{aligned}\right.
\end{aligned}
$$

Notemos que $\varphi_{\#}^{\prime}: \mathbb{Z} \rightarrow \mathbb{Z} \oplus \mathbb{Z} \oplus \mathbb{Z}$ é tal que $\varphi_{\#}^{\prime}(1)=(1,1,1), \quad\left(\psi^{\prime \prime} \circ\right.$ $\left.\varphi^{\prime}\right)_{\#}(1)=(1,2)$ e $\left(\psi^{\prime} \circ \psi^{\prime \prime} \circ \varphi^{\prime}\right)_{\#}(1)=3$, donde $h \circ \psi^{\prime} \circ \psi^{\prime \prime} \circ \varphi^{\prime} \simeq 3 h$. Logo, $\Theta\left([h],\left[h^{\prime}\right]\right)=\left[\psi \circ\left(h^{\prime} \vee h\right) \circ \varphi\right]$, onde $\left(\psi \circ\left(h^{\prime} \vee h\right) \circ \varphi\right) \circ p_{\mathbb{R} P(3)} \simeq 3 h$. A partir de construções análogas, consideremos o diagrama geométrico abaixo.

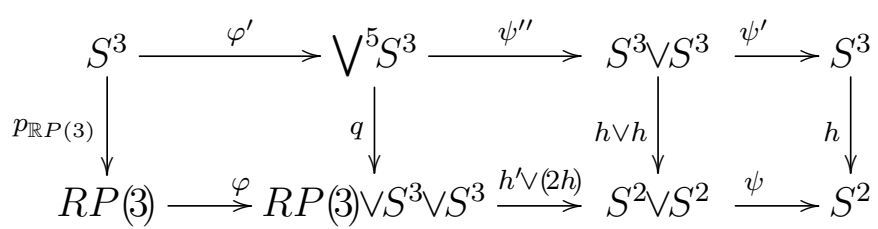

Notemos que $\varphi_{\#}^{\prime}: \mathbb{Z} \rightarrow \mathbb{Z}^{5}$ é tal que $\varphi_{\#}^{\prime}(1)=(1,1,1,1,1),\left(\psi^{\prime \prime} \circ \varphi^{\prime}\right)_{\#}(1)=$ $(1,4)$ e $\left(\psi^{\prime} \circ \psi^{\prime \prime} \circ \varphi^{\prime}\right)_{\#}(1)=5$, donde $h \circ \psi^{\prime} \circ \psi^{\prime \prime} \circ \varphi^{\prime} \simeq 5 h$. Logo, $\Theta\left([2 h],\left[h^{\prime}\right]\right)=$ $\left[\psi \circ\left(h^{\prime} \vee(2 h)\right) \circ \varphi\right]$, onde $\left(\psi \circ\left(h^{\prime} \vee h\right) \circ \varphi\right) \circ p_{\mathbb{R} P(3)} \simeq 5 h$. Analogamente, $\Theta\left([n h],\left[h^{\prime}\right]\right)=\left[\psi \circ\left(h^{\prime} \vee(n h)\right) \circ \varphi\right]$, onde $\left(\psi \circ\left(h^{\prime} \vee(n h)\right) \circ \varphi\right) \circ p_{\mathbb{R} P(3)} \simeq(2 n+1) h$, conforme o diagrama geométrico a seguir.

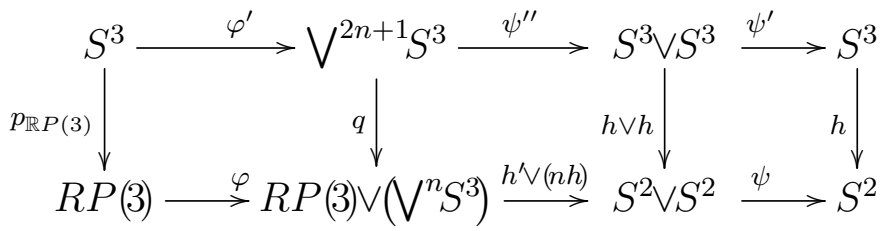

Seja $h_{2 n+1}^{\prime}:=\psi \circ\left(h^{\prime} \vee(n h)\right) \circ \varphi$. Desta forma, $\left[h_{2 n+1}^{\prime}\right]$ é o único elemento na órbita de $h^{\prime}$ tal que $h_{2 n+1}^{\prime} \circ p_{\mathbb{R} P(3)} \simeq(2 n+1) h$. Como a órbita de $h^{\prime}$ é igual a $i^{*-1}\left(\left[k_{2}\right]\right)$ e $\left[h^{\prime} \circ a_{2 n+1}\right] \in i^{*-1}\left(\left[k_{2}\right]\right)$ é tal que $h^{\prime} \circ a_{2 n+1}^{\prime} \circ p_{\mathbb{R} P(3)} \simeq(2 n+1) h$, segue, em particular, que $h_{2 n+1}^{\prime} \simeq h^{\prime} \circ a_{2 n+1}^{\prime}$. Logo, $i^{*-1}\left(\left[k_{2}\right]\right)=\left\{\left[h^{\prime} \circ a_{2 n+1}^{\prime}\right] \mid n \in \mathbb{Z}\right\}$.

A partir destas verificações, enunciamos o resultado a seguir. 
Teorema 196. Temos que $\left[\mathbb{R} P(3), S^{2}\right]=\mathbb{Z}_{(0)} \cup \dot{\mathbb{Z}}(1)$, onde

$$
\left\{\begin{array}{l}
\mathbb{Z}_{(0)}:=\left(i^{*}\right)^{-1}(0)=\left\{\left[(n h) \circ k_{3}\right] \mid n \in \mathbb{Z}\right\} \\
\mathbb{Z}_{(1)}:=\left(i^{*}\right)^{-1}\left(\left[k_{2}\right]\right)=\left\{\left[h^{\prime} \circ a_{2 n+1}^{\prime}\right] \mid n \in \mathbb{Z}\right\}
\end{array}\right.
$$

e $i: \mathbb{R} P(2) \hookrightarrow \mathbb{R} P(3)$ é a inclusão.

Proposição 197. Temos que $p_{\mathbb{R} P(3)}^{*}:\left[\mathbb{R} P(3), S^{2}\right] \rightarrow \pi_{3}\left(S^{2}\right)$ é uma bijeção.

Demonstração. De fato, dado $2 n[h] \in \pi_{3}\left(S^{2}\right)$, existe único $\left[(n h) \circ k_{3}\right]$ tal que $p_{\mathbb{R} P(3)}^{*}\left(\left[(n h) \circ k_{3}\right]\right)=2 n[h]$ e dado $(2 n+1)[h] \in \pi_{3}\left(S^{2}\right)$, existe único $\left[h^{\prime} \circ a_{2 n+1}^{\prime}\right]$ tal que $p_{\mathbb{R} P(3)}^{*}\left(\left[h^{\prime} \circ a_{2 n+1}^{\prime}\right]\right)=(2 n+1)[h]$, para todo $n \in \mathbb{Z}$.

\subsubsection{Cálculos do invariante}

Nesta subseção, vamos estudar o invariante $\tilde{\omega}\left(y_{0}, f\right)$ e o grupo em que ele se encontra, $\Omega_{1}^{f r}\left(E\left(y_{0}, f\right)\right)$, para as duas famílias de classes de homotopia de aplicações contínuas de $\mathbb{R} P(3)$ em $S^{2}, \mathbb{Z}_{(0)}$ e $\mathbb{Z}_{(1)}$, encontradas na subseção anterior.

Inicialmente, vamos determinar o grupo $H_{1}\left(E\left(y_{0}, f\right) ; \mathbb{Z}\right)$ para cada família. O resultado abaixo faz isto para a família $\mathbb{Z}_{(0)}$.

Proposição 198. Se $[f] \in \mathbb{Z}_{(0)}$, então $H_{1}\left(E\left(y_{0}, f\right) ; \mathbb{Z}\right) \cong \pi_{1}\left(E\left(y_{0}, f\right)\right) \cong$ $\mathbb{Z} \oplus \mathbb{Z}_{2}$.

Demonstração. De fato, desde que $[f] \in \mathbb{Z}_{(0)}$, então $f_{\mid \mathbb{R} P(2)}=f \circ i$ é homotópica à aplicação constante de $\mathbb{R} P(2)$ em $S^{2}$. Sem perda de generalidade, seja $f \circ i=c$ constante. Consideremos o seguinte diagrama geométrico

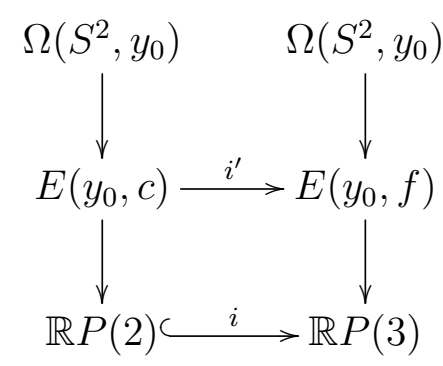


onde $i^{\prime}(x, \theta)=(i(x), \theta)$. Usando, agora, a sequência de homotopia da fibração, consideremos o diagrama algébrico induzido abaixo.

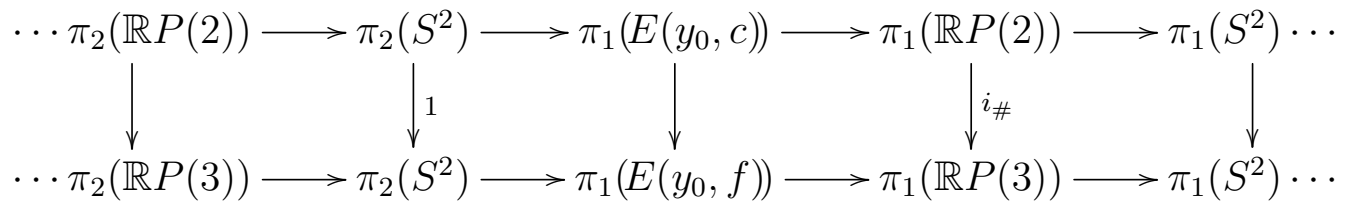

Como $\pi_{2}(\mathbb{R} P(3)) \cong \pi_{2}\left(S^{3}\right)=1$ e $\pi_{1}\left(S^{2}\right)=1$, então, na verdade, obtemos o seguinte diagrama algébrico:

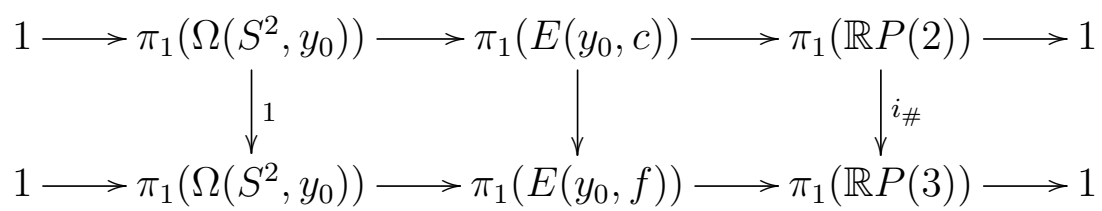

Agora, desde que $i_{\#}$ é isomorfismo, segue, pelo lema dos cinco, que $\pi_{1}\left(E\left(y_{0}, f\right)\right) \cong \pi_{1}\left(E\left(y_{0}, c\right)\right)$. Daí, como $E\left(y_{0}, c\right) \approx \mathbb{R} P(2) \times \Omega\left(S^{2}, y_{0}\right)$, pois $c$ é constante, então $\pi_{1}\left(E\left(y_{0}, f\right)\right) \cong \pi_{1}(\mathbb{R} P(2)) \oplus \pi_{1}\left(\Omega\left(S^{2}, y_{0}\right)\right) \cong \mathbb{Z}_{2} \oplus \mathbb{Z}$. $\operatorname{Logo}, H_{1}\left(E\left(y_{0}, f\right) ; \mathbb{Z}\right) \cong \pi_{1}\left(E\left(y_{0}, f\right)\right) \cong \mathbb{Z} \oplus \mathbb{Z}_{2}$, uma vez que $\pi_{1}\left(E\left(y_{0}, f\right)\right)$ é abeliano.

O próximo resultado determina o grupo para a família $\mathbb{Z}_{(1)}$.

Proposição 199. Se $[f] \in \mathbb{Z}_{(1)}$, então $H_{1}\left(E\left(y_{0}, f\right) ; \mathbb{Z}\right) \cong \pi_{1}\left(E\left(y_{0}, f\right)\right) \cong \mathbb{Z}$.

Demonstração. Com efeito, desde que $[f] \in \mathbb{Z}_{(1)}$, então $f_{\mid \mathbb{R} P(2)}=f \circ i$ é homotópica à aplicação colapsante, $k_{2}$, de $\mathbb{R} P(2)$ em $S^{2}$. Sem perda de generalidade, seja $f \circ i=k_{2}$. Consideremos o seguinte diagrama geométrico

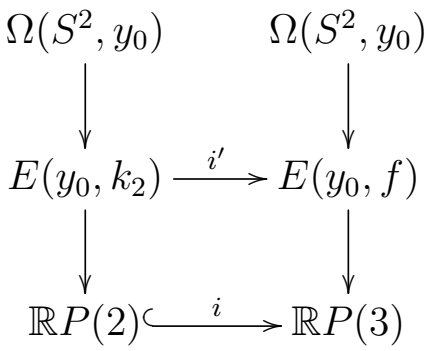


onde $i^{\prime}(x, \theta)=(i(x), \theta)$. Usando a sequência de homotopia da fibração, consideremos o diagrama algébrico induzido abaixo.

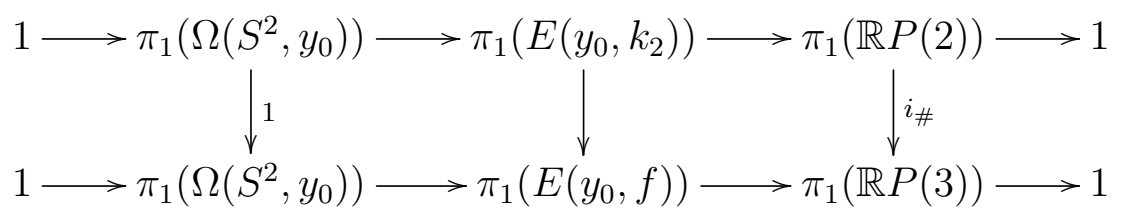

Como $i_{\#}$ é isomorfismo, segue, pelo lema dos cinco, que $\pi_{1}\left(E\left(y_{0}, f\right)\right) \cong$ $\pi_{1}\left(E\left(y_{0}, k_{2}\right)\right)$. Suponhamos, por ora, que $\pi_{1}\left(E\left(y_{0}, k_{2}\right)\right)$ é isomorfo a $\mathbb{Z}$. Logo, $H_{1}\left(E\left(y_{0}, f\right) ; \mathbb{Z}\right) \cong \pi_{1}\left(E\left(y_{0}, f\right)\right) \cong \mathbb{Z}$, uma vez que $\pi_{1}\left(E\left(y_{0}, f\right)\right)$ é abeliano. A demonstração de que $\pi_{1}\left(E\left(y_{0}, k_{2}\right)\right)$ é isomorfo a $\mathbb{Z}$ é dada no lema a seguir.

Lema 200. Temos que $\pi_{1}\left(E\left(y_{0}, k_{2}\right)\right) \cong \mathbb{Z}$.

Demonstração. Com efeito, consideremos a sequência exata curta abaixo.

$$
1 \rightarrow \pi_{1}\left(\Omega\left(S^{2}, y_{0}\right)\right) \stackrel{j_{\#}}{\rightarrow} \pi_{1}\left(E\left(y_{0}, k_{2}\right)\right) \stackrel{p r_{\#}}{\rightarrow} \pi_{1}(\mathbb{R} P(2)) \rightarrow 1
$$

Algebricamente, temos que $\pi_{1}\left(E\left(y_{0}, k_{2}\right)\right) \in\left\{\mathbb{Z}, \mathbb{Z} \oplus \mathbb{Z}_{2}, \mathbb{Z}_{2} * \mathbb{Z}_{2}\right\}$. Sejam $B_{1} \subset \mathbb{R}^{2}$ a bola aberta de centro 0 e raio 1 e $\overline{B_{1}} \subset \mathbb{R}^{2}$ a bola fechada de centro 0 e raio 1 . Consideremos as identificações $\mathbb{R} P(2)=\frac{\overline{B_{1}}}{x \sim_{S^{1}}-x}$ e $S^{2}=\frac{\overline{B_{1}}}{S^{1}}$. Tome $x_{0} \in k_{2}^{-1}\left(y_{0}\right), x_{0}=\overline{(0,1)}$. Seja $\alpha: I \rightarrow \mathbb{R} P(2)$,

$$
\alpha(t)=\overline{\left(\cos \left(\frac{\pi}{2}(2 t-1)\right), \operatorname{sen}\left(\frac{\pi}{2}(2 t-1)\right)\right)},
$$

um representante do gerador de $\pi_{1}\left(\mathbb{R} P(2), x_{0}\right) \cong \mathbb{Z}_{2}$. Para cada $t \in I$, seja $\theta_{t}: I \rightarrow S^{2}$ um laço baseado em $y_{0}$, onde $\theta_{0}=\theta_{1}=y_{0}$, onde o interior da região limitada por $\theta_{t}$ contém $\theta_{t-\varepsilon}$, para todo $0<t<1$, para todo $\varepsilon>0$ e onde cada $\theta_{t}$ tem a mesma orientação, no sentido horário, como sugerido em um dos laços na figura de $\overline{B_{1}}$ abaixo. Desta forma, notemos que $\Theta: I \rightarrow \Omega\left(S^{2}, y_{0}\right), \Theta(t)=\theta_{t}$, representa o gerador de $\pi_{1}\left(\Omega\left(S^{2}, y_{0}\right), y_{0}\right) \cong \mathbb{Z}$. 
Consideremos, agora, laços $\alpha^{\prime}, \Theta^{\prime}: I \rightarrow E\left(y_{0}, k_{2}\right)$, baseados em $\left(x_{0}, y_{0}\right)$, dados por $\alpha^{\prime}(t)=\left(\alpha(t), y_{0}\right)$ e $\Theta^{\prime}(t)=\left(x_{0}, \theta_{t}\right)$. Suponhamos, por ora, que o laço $\alpha^{\prime} * \alpha^{\prime} * \Theta^{\prime}$ é nulo-homotópico. Daí, $j_{\#}\left([\Theta]^{-1}\right)=\left[\Theta^{\prime}\right]^{-1}=2\left[\alpha^{\prime}\right]$. Afirmamos que $\pi_{1}\left(E\left(y_{0}, k_{2}\right)\right) \cong \mathbb{Z}$. Com efeito, primeiramente, suponhamos por absurdo que $\pi_{1}\left(E\left(y_{0}, k_{2}\right)\right) \cong \mathbb{Z} \oplus \mathbb{Z}_{2}$. Então algebricamente $j_{\#}(1)=$ $(2 k, 0)$, para algum $k \in \mathbb{Z}, k \neq 0$. Daí, $\frac{\pi_{1}\left(E\left(y_{0}, k_{2}\right)\right)}{\operatorname{Im} j_{\#}} \cong \mathbb{Z}_{2 k} \oplus \mathbb{Z}_{2}$, para algum $k \in \mathbb{Z}, k \neq 0$, o que não ocorre uma vez que $\frac{\pi_{1}\left(E\left(y_{0}, k_{2}\right)\right)}{\operatorname{Im} j_{\#}}=\frac{\pi_{1}\left(E\left(y_{0}, k_{2}\right)\right)}{\operatorname{ker} r_{\#}} \cong$ $\mathbb{Z}_{2}$. Agora, suponhamos por absurdo que $\pi_{1}\left(E\left(y_{0}, k_{2}\right)\right) \cong \mathbb{Z}_{2} * \mathbb{Z}_{2}$. Seja $\pi_{1}\left(E\left(y_{0}, k_{2}\right)\right)=<a, b \mid a^{2}, b^{2}>$. Notemos que os elementos de $\pi_{1}\left(E\left(y_{0}, k_{2}\right)\right)$ são da forma $a b a . \cdots . b a b$ ou são da forma $a b a . \cdots b a$ (ou os análogos começando com $b$ ), pois $a^{2}=1$ e $b^{2}=1$. A seguir, usamos apenas os começando com $a$, sendo que os argumentos são análogos para os começando com $b$. Como $(a b a . \cdots b a)^{2}=1$, então algebricamente $j_{\#}(1)=(a b a . \cdots b a b)^{2}$, uma vez que $j_{\#}$ é injetor. Primeiramente, seja $j_{\#}(1)=(a b)^{2}$. Então $\mathbb{Z}_{2} \cong \frac{\pi_{1}\left(E\left(y_{0}, k_{2}\right)\right)}{\operatorname{Im} j_{\#}}=$ $<a, b \mid a^{2}, b^{2},(a b)^{2}>=\{1, a, b, a b\}$, o qual é isomorfo a $\mathbb{Z}_{2} \oplus \mathbb{Z}_{2}$, o que é um absurdo. De modo geral, se $j_{\#}(1)=(a b)^{2 k}$, para algum $k \in \mathbb{Z}, k \geqslant 1$, então $\mathbb{Z}_{2} \cong \frac{\pi_{1}\left(E\left(y_{0}, k_{2}\right)\right)}{\operatorname{Im} j_{\#}}$ deveria ter $4 k$ elementos, o que não ocorre. Portanto, de fato, $\pi_{1}\left(E\left(y_{0}, k_{2}\right)\right) \cong \mathbb{Z}$.

A fim de mostrarmos que $\alpha^{\prime} * \alpha^{\prime} * \Theta^{\prime}$ é nulo-homotópico, consideremos 
a fibração $\Omega\left(S^{2} ; S, N\right) \rightarrow E\left(S, k_{2}\right) \rightarrow \mathbb{R} P(2)$, onde $S=k_{2}(\overline{(0,0)}), N=$ $k_{2}\left(x_{0}\right)=y_{0}$ são os pólos sul e norte de $S^{2} \subset \mathbb{R}^{3}$, respectivamente. Para cada $t \in I$, seja $\gamma_{t}: I \rightarrow S^{2}$ a geodésica que começa em $S$, termina em $N$ e passa por $(\cos (2 \pi t), \operatorname{sen}(2 \pi t), 0) \in \mathbb{R}^{3}$. Assim, observemos que $\Gamma: I \rightarrow$ $\Omega\left(S^{2} ; S, N\right), \Gamma(t)=\gamma_{t}$, representa o gerador de $\pi_{1}\left(\Omega\left(S^{2} ; S, N\right), \gamma_{0}\right) \cong \mathbb{Z}$. Consideremos os laços $\alpha^{\prime \prime}, \Gamma^{\prime}: I \rightarrow E\left(S, k_{2}\right)$, baseados em $\left(x_{0}, \gamma_{0}\right)$, dados por $\alpha^{\prime \prime}(t)=\left(\alpha(t), \gamma_{0}\right)$ e $\Gamma^{\prime}(t)=\left(x_{0}, \gamma_{t}\right)$. Vamos mostrar que o laço $\alpha^{\prime \prime} * \alpha^{\prime \prime} * \Gamma^{\prime}$ é livremente nulo-homotópico. De fato, $H: I \times I \rightarrow E\left(S, k_{2}\right)$, $H(s, t)=\left(\overline{\left(s \cos \left(\pi\left(2 t-\frac{1}{2}\right)\right), s \operatorname{sen}\left(\pi\left(2 t-\frac{1}{2}\right)\right)\right)}, \gamma_{t, s}\right)$, onde $\gamma_{t, s}$ é o caminho sobre a geodésica $\gamma_{t}$ que começa em $S$ e termina em $\gamma_{t}(s)$, é uma homotopia livre entre $c_{(\overline{(0,0), S)}}$ e $\alpha^{\prime \prime} * \alpha^{\prime \prime} * \Gamma^{\prime}$. Daí, $c_{\left(x_{0}, \gamma_{0}\right)}$ e $\alpha^{\prime \prime} * \alpha^{\prime \prime} * \Gamma^{\prime}$ são homotópicos, com extremos fixos. Contraindo $\gamma_{0}$ em $y_{0}$, obtemos que $c_{\left(x_{0}, y_{0}\right)}$ e $\alpha^{\prime} * \alpha^{\prime} * \Theta^{\prime}$ são homotópicos, com extremos fixos.

Com o próximo resultado, vamos decompor o grupo de bordismo em soma direta e descrever o invariante $\tilde{\omega}\left(y_{0}, f\right)$ em termos desta decomposição.

Proposição 201. Sejam $f: \mathbb{R} P(3) \rightarrow S^{2}$ uma aplicação contínua e $y_{0} \in S^{2}$. Existe um isomorfismo $\varphi: \Omega_{1}^{f r}\left(E\left(y_{0}, f\right)\right) \rightarrow \mathbb{Z}_{2} \oplus H_{1}\left(E\left(y_{0}, f\right) ; \mathbb{Z}\right)$ tal que $\varphi([M, \tilde{g}, \bar{g}])=\left([M\right.$, cte, $\left.\bar{g}], \tilde{g}_{*}([M])\right)$, onde $[M] \in H_{1}(M ; \mathbb{Z})$ denota a classe fundamental de $M$.

Demonstração. De fato, pela observação 193 , notemos que $E\left(y_{0}, f\right)$ é um CW-complexo conexo por caminhos tal que $\pi_{n}\left(E\left(y_{0}, f\right)\right)$ é finitamente gerado, para todo $n \geqslant 1$. Daí, como, pelas proposições 198 e 199, $\pi_{1}\left(E\left(y_{0}, f\right)\right) \in$ $\left\{\mathbb{Z}, \mathbb{Z} \oplus \mathbb{Z}_{2}\right\}$, então, pelo lema $49, E\left(y_{0}, f\right)$ tem o mesmo tipo de homotopia de um CW-complexo de tipo finito. Logo, pelo teorema 110 e pela observação 111, sendo $\varphi$ trivial, segue que a sequência

$$
\cdots H_{2}\left(E\left(y_{0}, f\right) ; \mathbb{Z}\right) \rightarrow \Omega_{1}^{f r} \stackrel{i_{*}}{\rightarrow} \Omega_{1}^{f r}\left(E\left(y_{0}, f\right)\right) \stackrel{\mu}{\rightarrow} H_{1}\left(E\left(y_{0}, f\right) ; \mathbb{Z}\right) \rightarrow 0
$$


é exata, onde $\Omega_{1}^{f r}:=\Omega_{1}^{f r}\left(a_{0}\right)$ e $i: a_{0} \hookrightarrow E\left(y_{0}, f\right)$ é a inclusão. Considere $r: E\left(y_{0}, f\right) \rightarrow a_{0}$ a aplicação constante. Desde que $r_{*} \circ i_{*}=1_{\Omega_{1}^{f r}}$, então a sequência acima cinde. Daí, definindo $\varphi: \Omega_{1}^{f r}\left(E\left(y_{0}, f\right)\right) \rightarrow \mathbb{Z}_{2} \oplus$ $H_{1}\left(E\left(y_{0}, f\right) ; \mathbb{Z}\right)$ por $\varphi([M, \tilde{g}, \bar{g}])=\left(r_{*}([M, \tilde{g}, \bar{g}]), \mu([M, \tilde{g}, \bar{g}])\right)$, temos que $\varphi$ é um isomorfismo, onde identificamos $\mathbb{Z}_{2} \cong \Omega_{1}^{f r}$ (vide o exemplo 109). Agora, notemos que $r_{*}([M, \tilde{g}, \bar{g}])=[M$, cte, $\bar{g}]$ e, pelo teorema $110, \mu([M, \tilde{g}, \bar{g}])=$ $\tilde{g}_{*}([M])$, donde o resultado segue.

Vamos analisar $\tilde{g}_{*}$ para cada família. Mas antes, vamos analisar alguns homomorfismos induzidos que irão nos ajudar nesta tarefa. Consideremos o seguinte diagrama geométrico

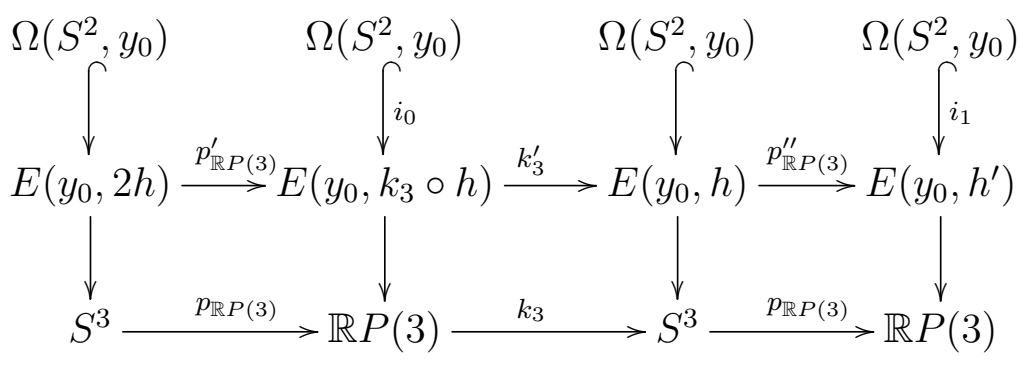

onde $p_{\mathbb{R} P(3)}^{\prime}, p_{\mathbb{R} P(3)}^{\prime \prime}:(x, \theta) \mapsto\left(p_{\mathbb{R} P(3)}(x), \theta\right)$ e $k_{3}^{\prime}:(x, \theta) \mapsto\left(k_{3}(x), \theta\right)$. Usando a sequência de homotopia da fibração para as linhas verticais, consideremos agora o diagrama algébrico induzido abaixo

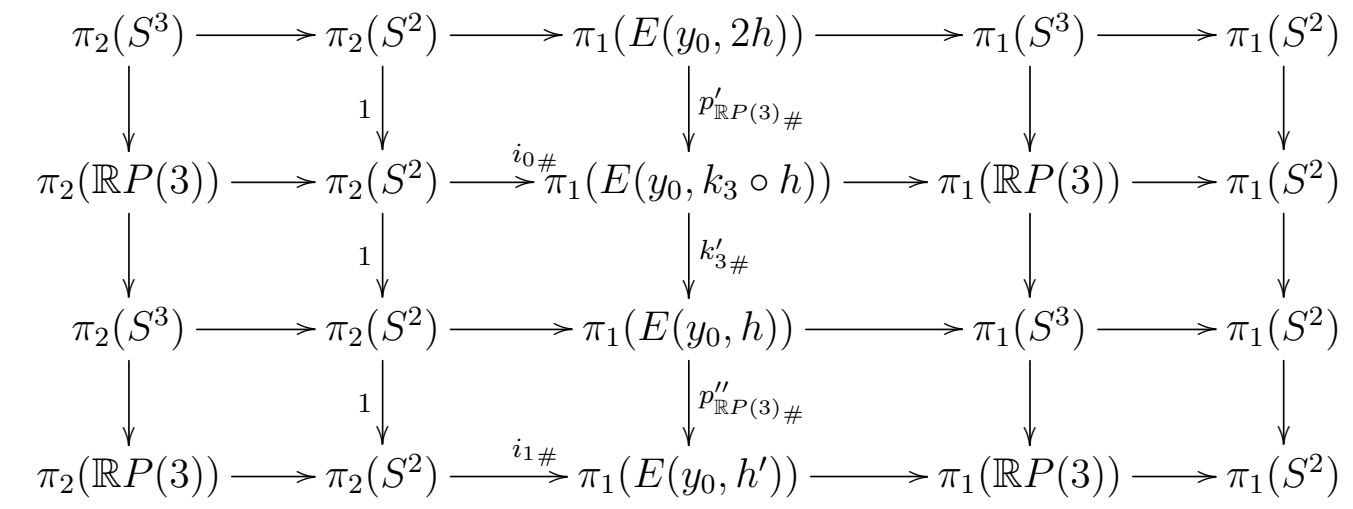

Como $\pi_{2}(\mathbb{R} P(3)) \cong \pi_{2}\left(S^{3}\right)=1, \pi_{2}\left(S^{2}\right) \cong \mathbb{Z}, \pi_{1}\left(S^{3}\right)=1, \pi_{1}(\mathbb{R} P(3)) \cong \mathbb{Z}_{2} \mathrm{e}$ 
$\pi_{1}\left(S^{2}\right)=1$, então obtemos o seguinte diagrama algébrico

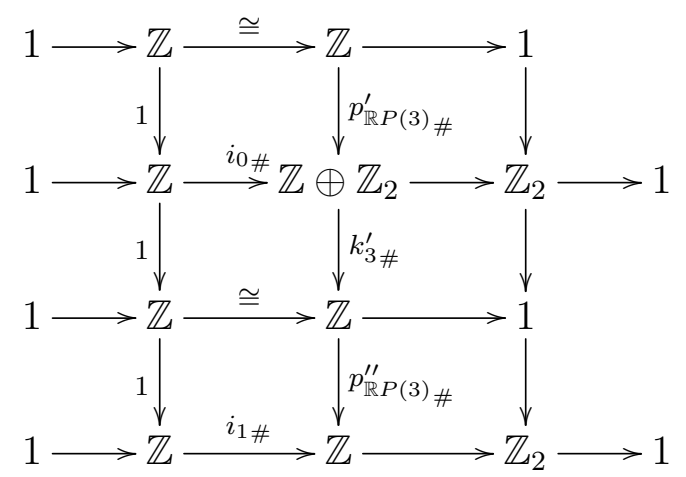

uma vez que $\pi_{1}\left(E\left(y_{0}, h\right)\right) \cong \mathbb{Z} \cong \pi_{1}\left(E\left(y_{0}, 2 h\right)\right)$ e, pelos resultados anteriores, $\pi_{1}\left(E\left(y_{0}, h^{\prime}\right)\right) \cong \mathbb{Z}$ e $\pi_{1}\left(E\left(y_{0}, k_{3} \circ h\right)\right) \cong \mathbb{Z} \oplus \mathbb{Z}_{2}$. Pela comutatividade do diagrama, desde que, algebricamente, $i_{1 \#}=\times 2$, então $p_{\mathbb{R} P(3) \#}^{\prime \prime}=\times 2$. Ainda, temos que $k_{3 \#}^{\prime} \circ p_{\mathbb{R} P(3)_{\#}}^{\prime}$ é um isomorfismo de $\mathbb{Z}$ em $\mathbb{Z}$. Desta forma, notemos que os homomorfismos induzidos no primeiro grupo de homologia com coeficientes em $\mathbb{Z}$ são tais que $p_{\mathbb{R} P(3)_{*}}^{\prime \prime}=\times 2$, e $k_{3 *}^{\prime} \circ p_{\mathbb{R} P(3)_{*}}^{\prime}$ é um isomorfismo de $\mathbb{Z}$ em $\mathbb{Z}$.

Os próximos resultados analisam $\tilde{g}_{*}$ para a família $\mathbb{Z}_{(1)}$.

Proposição 202. Seja $\tilde{g}: h^{\prime-1}\left(y_{0}\right) \rightarrow E\left(y_{0}, h^{\prime}\right)$ dada por $\tilde{g}(x)=\left(x, y_{0}\right)$. Então $\tilde{g}_{*}: H_{1}\left(h^{\prime-1}\left(y_{0}\right) ; \mathbb{Z}\right) \rightarrow H_{1}\left(E\left(y_{0}, h^{\prime}\right) ; \mathbb{Z}\right)$ é um homomorfismo injetor de $\mathbb{Z}$ em $\mathbb{Z}$.

Demonstração. De fato, consideremos o seguinte diagrama geométrico

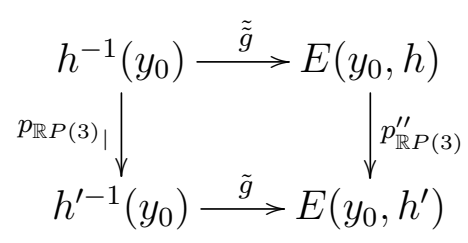

onde $\tilde{\tilde{g}}: x \mapsto\left(x, y_{0}\right)$ e $p_{\mathbb{R} P(3)}^{\prime \prime}:(x, \theta) \mapsto\left(p_{\mathbb{R} P(3)}(x), \theta\right)$. Como, pela observação 36 , podemos identificar $h^{-1}\left(y_{0}\right) \operatorname{com} S^{1}$, então, pela construção de $h^{\prime}$, podemos identificar $h^{\prime-1}\left(y_{0}\right)$ com $S^{1}$. Em particular, obtemos o diagrama algébrico 
abaixo, induzido, do diagrama acima, no primeiro grupo de homologia com coeficientes em $\mathbb{Z}$.

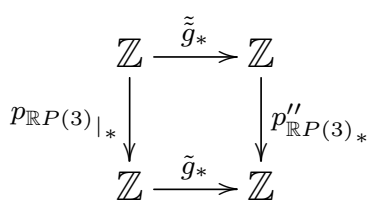

Desde que, pela proposição $151, \omega^{\prime \prime}([h])= \pm 1$, então $\tilde{\tilde{g}}_{*}=\times( \pm 1)$. Logo, pelas considerações acima, $\left(p_{\mathbb{R} P(3)}^{\prime \prime} \circ \tilde{\tilde{g}}\right)_{*}=\times( \pm 2)$. Daí, $\tilde{g}_{*}$ é injetor.

Proposição 203. Seja $\tilde{g}:\left(h^{\prime} \circ a_{2 n+1}^{\prime}\right)^{-1}\left(y_{0}\right) \rightarrow E\left(y_{0}, h^{\prime} \circ a_{2 n+1}^{\prime}\right)$ dada por $\tilde{g}(x)=\left(x, y_{0}\right)$. Então $\tilde{g}_{*}: H_{1}\left(\left(h^{\prime} \circ a_{2 n+1}^{\prime}\right)^{-1}\left(y_{0}\right) ; \mathbb{Z}\right) \rightarrow H_{1}\left(E\left(y_{0}, h^{\prime} \circ a_{2 n+1}^{\prime}\right) ; \mathbb{Z}\right)$ é um homomorfismo injetor de $\mathbb{Z}$ em $\mathbb{Z}$.

Demonstração. Com efeito, consideremos o seguinte diagrama geométrico

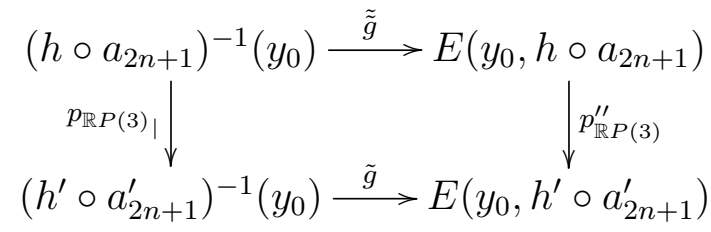

onde $\tilde{\tilde{g}}: x \mapsto\left(x, y_{0}\right)$ e $p_{\mathbb{R} P(3)}^{\prime \prime}:(x, \theta) \mapsto\left(p_{\mathbb{R} P(3)}(x), \theta\right)$. Notemos que, pela observação 194 , podemos identificar tanto $\left(h \circ a_{2 n+1}\right)^{-1}\left(y_{0}\right)$, quanto $\left(h^{\prime} \circ\right.$ $\left.a_{2 n+1}^{\prime}\right)^{-1}\left(y_{0}\right)$, com $S^{1}$. Daí, obtemos o diagrama algébrico abaixo, induzido, do diagrama acima, no primeiro grupo de homologia com coeficientes em $\mathbb{Z}$.

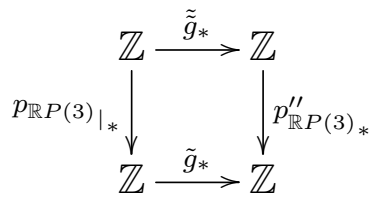

Como, pela proposição 151 , temos que $\omega^{\prime \prime}((2 n+1)[h])= \pm(2 n+1)$, então $\tilde{\tilde{g}}_{*}=\times( \pm(2 n+1))$. Portanto, por argumentos análogos às considerações acima, $\left(p_{\mathbb{R} P(3)}^{\prime \prime} \circ \tilde{\tilde{g}}\right)_{*}=\times( \pm 2(2 n+1))$. Daí, $\tilde{g}_{*}$ é injetor.

O resultado a seguir analisa $\tilde{g}_{*}$ para a família $\mathbb{Z}_{(0)}$. 
Proposição 204. Seja $n \neq 0$ e seja $\tilde{g}:\left((n h) \circ k_{3}\right)^{-1}\left(y_{0}\right) \rightarrow E\left(y_{0},(n h) \circ k_{3}\right)$ dada por $\tilde{g}(x)=\left(x, y_{0}\right)$. Então $\tilde{g}_{*}: H_{1}\left(\left((n h) \circ k_{3}\right)^{-1}\left(y_{0}\right) ; \mathbb{Z}\right) \rightarrow H_{1}\left(E\left(y_{0},(n h) \circ k_{3}\right) ; \mathbb{Z}\right)$ é um homomorfismo injetor de $\mathbb{Z}$ em $\mathbb{Z} \oplus \mathbb{Z}_{2}$.

Demonstração. Consideremos o diagrama geométrico

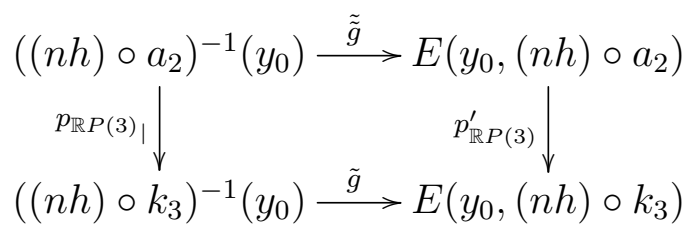

onde $\tilde{\tilde{g}}: x \mapsto\left(x, y_{0}\right)$ e $p_{\mathbb{R} P(3)}^{\prime}:(x, \theta) \mapsto\left(p_{\mathbb{R} P(3)}(x), \theta\right)$. Seja $n \neq 0$. Como $M C C\left(y_{0},(2 n) h\right)=1$, então, via bordismo, podemos identificar $\left((n h) \circ a_{2}\right)^{-1}\left(y_{0}\right)$ com $S^{1}$. Agora, pela observação 195, podemos identificar $\left((n h) \circ k_{3}\right)^{-1}\left(y_{0}\right)$ com $S^{1}$. Assim, obtemos o diagrama algébrico abaixo, induzido, do diagrama acima, no primeiro grupo de homologia com coeficientes em $\mathbb{Z}$.

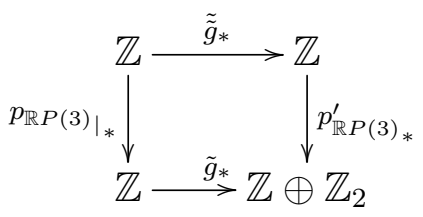

Desde que, pela proposição $151, \omega^{\prime \prime}((2 n)[h])= \pm(2 n)$, então $\tilde{\tilde{g}}_{*}=\times( \pm(2 n))$. Logo, por argumentos análogos às considerações acima, $\left(p_{\mathbb{R} P(3)}^{\prime \prime} \circ \tilde{\tilde{g}}\right)_{*}$ é injetor. Logo, $\tilde{g}_{*}$ é injetor.

O próximo resultado utiliza os resultados anteriores para estudar o anulamento de $\tilde{\omega}\left(y_{0}, f\right)$.

Teorema 205. Seja $f: \mathbb{R} P(3) \rightarrow S^{2}$ uma aplicação contínua. Se $f \simeq$ $h^{\prime} \circ a_{2 n+1}^{\prime}$, então $\tilde{\omega}\left(y_{0}, f\right) \neq 0$. Se $f \simeq(n h) \circ k_{3}$, então $\tilde{\omega}\left(y_{0}, f\right)=0$ se, e somente se, $n=0$, isto é, $f \simeq y_{0}$.

Demonstração. De fato, pela proposição 201, a menos de isomorfismo, temos que $\tilde{\omega}\left(y_{0}, f\right)=\left([M\right.$, cte, $\left.\bar{g}], \tilde{g}_{*}([M])\right)$, onde $[M] \in H_{1}(M ; \mathbb{Z})$ denota a 
classe fundamental de $M$. Se $f \simeq h^{\prime} \circ a_{2 n+1}^{\prime}$, então, pela proposição $203, \tilde{g}_{*}$ é injetor, donde $\tilde{g}_{*}([M]) \neq 0$ e portanto $\tilde{\omega}\left(y_{0}, f\right) \neq 0$. Da mesma forma, se $f \simeq(n h) \circ k_{3}$, com $n \neq 0$, então, pela proposição 204, $\tilde{g}_{*}$ é injetor, donde $\tilde{g}_{*}([M]) \neq 0$ e portanto $\tilde{\omega}\left(y_{0}, f\right) \neq 0$. Finalmente, se $f \simeq(n h) \circ k_{3}$, com $n=0$, então $\left(y_{0}, f\right)$ é loose e portanto $\tilde{\omega}\left(y_{0}, f\right)=0$.

Teorema 206. Seja $f: \mathbb{R} P(3) \rightarrow S^{2}$ uma aplicação contínua. Então

$$
M C C\left(y_{0}, f\right)=\tilde{N}\left(y_{0}, f\right)= \begin{cases}0, & \text { se } f \simeq y_{0}, \\ 1, & \text { caso contrário. }\end{cases}
$$

Demonstração. Com efeito, desde que o contradomínio de $f$ é $S^{2}$, então $f$ é deformável a livre de raiz se, e só se, $f$ é homotópica a aplicação constante. Em particular, se $f \not y_{0}$, segue que $M C C\left(y_{0}, f\right) \geqslant 1$ e, se $f \simeq y_{0}$, temos que $\operatorname{MCC}\left(y_{0}, f\right)=0=\tilde{N}\left(y_{0}, f\right)$. Seja $f \not y_{0}$. Como, pelas observações $194 \mathrm{e}$ 195 , temos que $\operatorname{MCC}\left(y_{0}, f\right) \leqslant 1$, então $M C C\left(y_{0}, f\right)=1$. Finalmente, desde que $E\left(y_{0}, f\right)$ é conexo por caminhos, então, pelo teorema $205, \tilde{N}\left(y_{0}, f\right)=1$ e o resultado segue. 


\section{Referências Bibliográficas}

[AHi-72] J. F. Adams; P. J. Hilton. On the chain algebra of a loop space. Comment. Math. Helv. 30, 1956, 305-330.

[BaBa-13] S. Basu; S. Basu. Homotopy groups and periodic geodesics of closed 4-manifolds. arXiv:1303.3328v1, 2013, 1-22.

[Broo-72] R. B. S. Brooks. On removing coincidences of two maps when only one, rather than both, of them may be deformed by a homotopy. Pacific J. Math. (1) 40, 1972, 45-52.

[Brow-71] R. F. Brown. The Lefschetz fixed point theorem. Scott, Foresman and Co., 1971.

[Brow-93] R. F. Brown. Wecken properties for manifolds. Contemp. Math. 152, American Mathematical Society, 1993.

[Brow-99] R. F. Brown. Fixed point theory. History of topology, NorthHolland, 1999, 271-299.

[DacRa-88] J. Daccach; D. Randall. Cobordismo normal e aplicações. Notas didáticas do ICMSC-USP, 1988.

[Dax-72] J. P. Dax. Étude homotopique des espaces de plongements. Ann. Sci. École Norm. Sup. (4) 5, 1972, 303-377. 
[DoGo-05] A. Dold; D. L. Gonçalves. Self-coincidence of fibre maps. Osaka J. Math. (2) 42, 2005, 291-307.

[EE-69] C. J. Earle; J. Eells. A fibre bundle description of Teichmüller theory. J. Differential Geometry 3, 1969, 19-43.

[FeOTa-08] Y. Félix; J. Oprea; D. Tanré. Algebraic models in geometry. Oxf. Grad. Texts Math. 17, Oxford Univ. Press, 2008.

[FrPi-90] R. Fritsch; R. A. Piccinini. Cellular structures in topology. Cambridge Stud. Adv. Math. 19, Cambridge University Press, 1990.

[Go-05] D. L. Gonçalves. Coincidence theory. Handbook of topological fixed point theory, Springer, 2005, 3-42.

[GoWo-05] D. L. Gonçalves; P. Wong. Wecken property for roots. Proc. Amer. Math. Soc. (9) 133, 2005, 2779-2782.

[GuPo-74] V. Guillemin; A. Pollack. Differential topology. Prentice-Hall, Inc., 1974.

[HaQ-74] A. Hatcher; F. Quinn. Bordism invariants of intersections of submanifolds. Trans. Amer. Math. Soc. 200, 1974, 327-344.

[Ha-02] A. Hatcher. Algebraic topology. Cambridge University Press, 2002.

[Ha-04] A. Hatcher. The classification of 3-manifolds - a brief survey. www.math.cornell.edu/ hatcher/Papers/3Msurvey.pdf, 2004.

[Ho-27] H. Hopf. Vektorfelder in n-dimensionalen Mannigfaltigkeiten. Math. Ann. (1) 96, 1927, 225-249. 
[Ho-29] H. Hopf. Über die algebraische Anzahl von Fixpunkten. Math. Z. (1) 29, 1929, 493-524.

[Hu-94] D. Husemoller. Fibre bundles. Third edition. Grad. Texts in Math. 20, Springer, 1994.

[JaWhj-54] I. M. James; J. H. C. Whitehead. The homotopy theory of sphere bundles over spheres. I. Proc. London Math. Soc. (3) 4, 1954, 196-218.

[JaWhj-55] I. M. James; J. H. C. Whitehead. The homotopy theory of sphere bundles over spheres. II. Proc. London Math. Soc. (3) $5,1955,148-166$.

[Ji-83] B. J. Jiang. Lectures on Nielsen fixed point theory. Contemp. Math. 14, American Mathematical Society, 1983.

[Ji-84] B. J. Jiang. Fixed points and braids. Invent. Math. (1) 75, 1984, 69-74.

[Ji-85] B. J. Jiang. Fixed points and braids. II. Math. Ann. (2) 272, 1985, 249-256.

[K-81] U. Koschorke. Vector fields and other vector bundle morphisms. A singularity approach. Lecture Notes in Math. 847, Springer, 1981.

[K-04] U. Koschorke. Selfcoincidences in higher codimensions. J. Reine Angew. Math. 576, 2004, 1-10.

[K-06] U. Koschorke. Nielsen coincidence theory in arbitrary codimensions. J. Reine Angew. Math. 598, 2006, 211-236. 
[K-062] U. Koschorke. Nonstabilized Nielsen coincidence invariants and Hopf-Ganea homomorphisms. Geom. Topol. 10, 2006, 619-666.

[K-07] U. Koschorke. Selfcoincidences and roots in Nielsen theory. $J$. Fixed Point Theory Appl. (2) 2, 2007, 241-259.

[K-08] U. Koschorke. Minimizing coincidence numbers of maps into projective spaces. Geom. Topol. Monogr. (2) 14, 2008, 373-391.

[K-11] U. Koschorke. Minimum numbers and Wecken theorems in topological coincidence theory. I. J. Fixed Point Theory Appl. (1) $10,2011,3-36$.

[L-23] S. Lefschetz. Continuous transformations of manifolds. Proc. Nat. Acad. Sci. U.S.A. (3) 9, 1923, 90-93.

[L-25] S. Lefschetz. Intersections of complexes on manifolds. Proc. Nat. Acad. Sci. U.S.A. (6) 11, 1925, 287-289.

[L-26] S. Lefschetz. Intersections and transformations of complexes and manifolds. Trans. Amer. Math. Soc. (1) 28, 1926, 1-49.

[L-27] S. Lefschetz. Manifolds with a boundary and their transformations. Trans. Amer. Math. Soc. (2) 29, 1927, 429-462.

[Ma-77] W. S. Massey. Algebraic topology. An introduction. Grad. Texts in Math. 56, Springer, 1977.

[Mi-58] J. W. Milnor. Differential topology. Lectures by John Milnor, Princeton Univ. - Notes by James Munkres, 1958.

[Mi-65] J. W. Milnor. Topology from the differentiable viewpoint. The University Press of Virginia, 1965. 
[MiSta-74] J. W. Milnor; J. D. Stasheff. Characteristic classes. Ann. of Math. Stud. 76, Princeton Univ. Press, 1974.

[MuShi-99] J. Mukai; T. Shinpo. Some homotopy groups of the mod 4 Moore space. J. Fac. Sci. Shinshu University (1) 34, 1999, $1-14$.

[N-27] J. Nielsen. Untersuchungen zur Topologie der geschlossenen zweiseitigen Flächen. Acta Math. (1) 50, 1927, 189-358.

[P-77] D. S. Passman. The algebraic structure of group rings. Pure Appl. Math., Wiley-Interscience, 1977.

[Re-36] K. Reidemeister. Automorphismen von Homotopiekettenringen. Math. Ann. (1) 112, 1936, 586-593.

[Sa-73] H. A. Salomonsen. Bordism and geometric dimension. Math. Scand. 32, 1973, 87-111.

[Sc-55] H. Schirmer. Mindestzahlen von Koinzidenzpunkten. J. Reine Angew. Math. 194, 1955, 21-39.

[Se-51] J. P. Serre. Homologie singulière des espaces fibrés. Applications. Ann. of Math. (2) 54, 1951, 425-505.

[Se-53] J. P. Serre. Groupes D'Homotopie Et Classes De Groupes Abeliens. Ann. of Math. (2) 58, 1953, 258-294.

[Sha-11] A. R. Shastri. Elements of differential topology. CRC Press, 2011.

[Sm-59] S. Smale. Diffeomorphisms of the 2-sphere. Proc. Amer. Math. Soc. 10, 1959, 621-626. 
[Sp-66] E. H. Spanier. Algebraic topology. McGraw-Hill Book Co., 1966.

[Sti-35] E. Stiefel. Richtungsfelder und Fernparallelismus in n-dimensionalen Mannigfaltigkeiten. Comment. Math. Helv. (1) 8, 1935, 305-353.

[Tod-62] H. Toda. Composition methods in homotopy groups of spheres. Ann. of Math. Stud. 49, Princeton Univ. Press, 1962.

[Tol-74] J. L. Tollefson. The compact 3-manifolds covered by S2xR1. Proc. Amer. Math. Soc. 45, 1974, 461-462.

[V-94] J. W. Vick. Homology theory. An introduction to algebraic topology. Second edition. Grad. Texts in Math. 145, Springer, 1994.

[Wa-65] C. T. C. Wall. Finiteness conditions for CW-complexes. Ann. of Math. (1) 81, 1965, 56-69.

[We-41] F. Wecken. Fixpunktklassen. I. Math. Ann. 117, 1941, 659-671.

[We-412] F. Wecken. Fixpunktklassen. II. Homotopieinvarianten der Fixpunkttheorie. Math. Ann. 118, 1941, 216-234.

[We-42] F. Wecken. Fixpunktklassen. III. Mindestzahlen von Fixpunkten. Math. Ann. 118, 1942, 544-577.

[Whg-78] G. W. Whitehead. Elements of homotopy theory. Grad. Texts in Math. 61, Springer, 1978. 